\title{
Application of Parent-Child UAV Tasking For Wildfire Detection and Response
}

\author{
A Thesis \\ Presented to \\ The Faculty of California Polytechnic State University, \\ San Luis Obispo
}

\author{
In Partial Fulfillment \\ Of the Requirements for the Degree \\ Master of Science in Aerospace Engineering
}

By:

Stephen T. Kubik

November 20, 2008 
(C) 2008

Stephen T. Kubik

ALL RIGHTS RESERVED 


\section{COMMITTEE MEMBERSHIP}

TITLE:

AUTHOR:

DATE SUBMITTED:

Nov 2008

Dr. Daniel J. Biezad

Thesis Advisor

Aeronautical Engineering

Cal Poly, San Luis Obispo

Application of Parent-Child UAV Tasking For Wildfire Detection and Response

Stephen T. Kubik

Chad R. Frost

Committee Member

NASA-Ames Research Center

Moffitt Field

Dr. Eric Mehiel

Committee Member

Aeronautical Engineering

Cal Poly, San Luis Obispo

Dr. Frank Owen

Committee Member

Mechanical Engineering

Cal Poly, San Luis Obispo 


\begin{abstract}
Application of Parent-Child UAV Tasking For Wildfire Detection and Response Stephen T. Kubik
\end{abstract}

In recent years, unmanned aerial vehicles (UAVs) have become a dominant force in the aerospace industry. Recent technological developments have moved these aircraft from remote operation roles to more active response missions. Of particular interest is the possibility of applying UAVs toward solving complex problems in long-endurance missions. Under that belief, the feasibility of utilizing UAVs for wildfire detection and response was investigated in a partnership that included NASA's Aeronautics Research Mission Directorate and Science Mission Directorate, and the United States Forest Service. Under NASA's Intelligent Mission Management (IMM) project, research was conducted to develop a mission architecture that would enable use of a high altitude UAV to search for reported wildfires with a separate low altitude UAV supporting ground assets.

This research proposes a "straw man" concept incorporating both a High Altitude Long Endurance (HALE) UAV and a Low Altitude Short Endurance (LASE) UAV in a loosely coupled, low cost solution tailored towards wildfire response. This report identifies the communications architecture, algorithms, and required system configuration that meets the outlined goals of the IMM project by mitigating wildfires and addressing the United States Forest Service immediate needs. The end product is a defined parent-child framework capable of meeting all wildfire mission goals. The concept has been implemented in simulation, the results of which are presented in this report. 


\section{ACKNOWLEDGEMENTS}

First, I would like to thank Dr. Daniel J. Biezad of Cal Poly, San Luis Obispo and Chad Frost of NASA-Ames Research Center for their unwavering support and guidance in helping me accomplish this project. Without their efforts and experience, this opportunity and research would never have become a possibility. Additionally, special recognition should be given to the various people who have been of assistance to me in this finalizing project at each stage of its development. These people include Rachelle Yandel, Lauren McLane, Keith Rothman, Robert Rivera, Chris Adan, Michael Kosman, the NASA Intelligent Systems Division and the U. S. Army Aeroflightdynamics Directorate at Ames Research Center, and my parents for their invaluable efforts and contributions in the completion of this thesis.

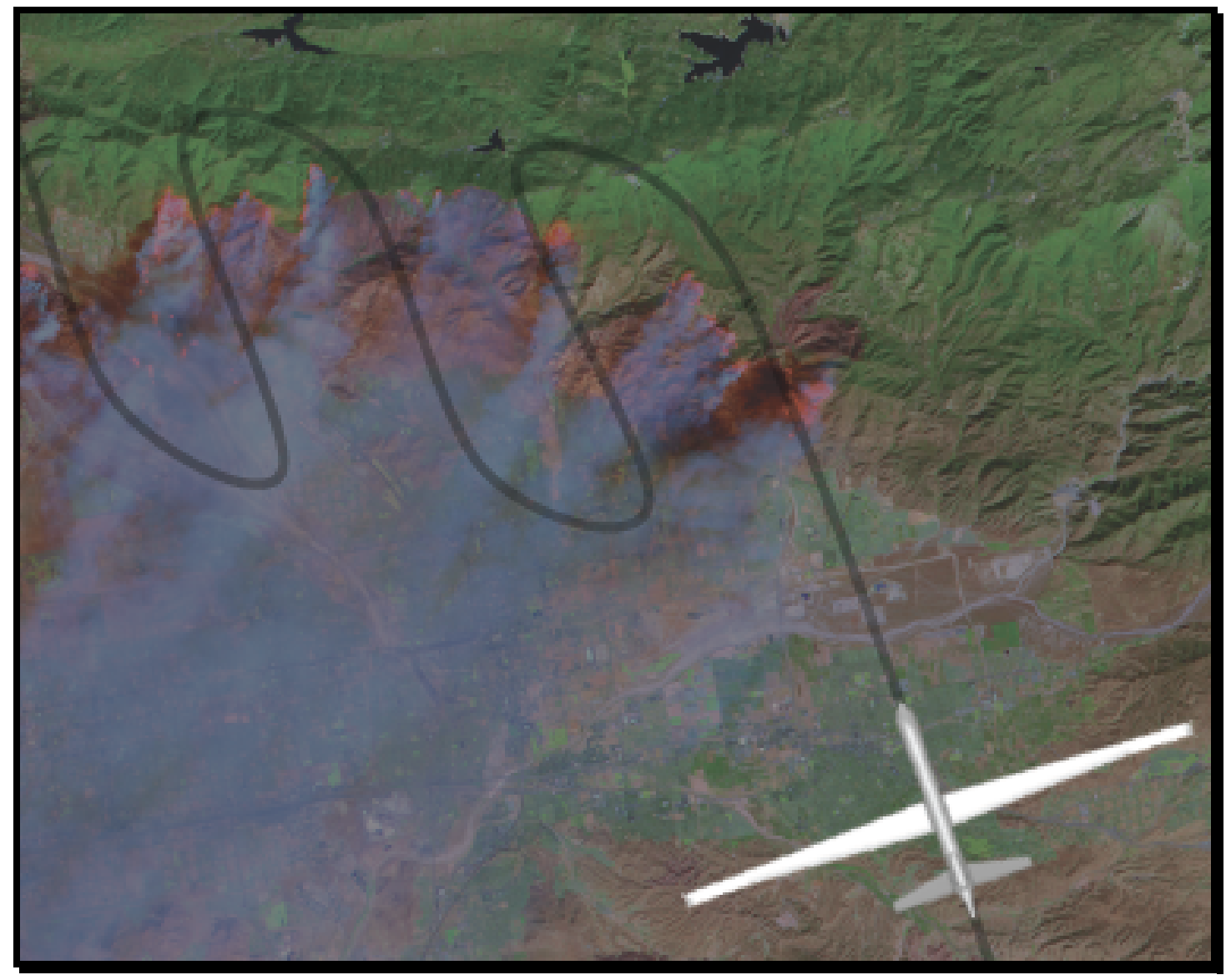




\section{TABLE OF CONTENTS}

Section Name

Page

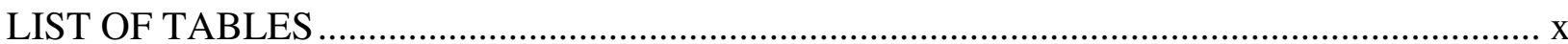

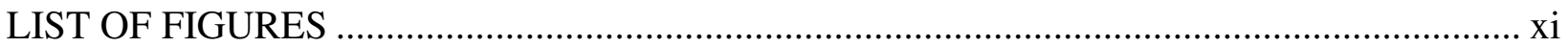

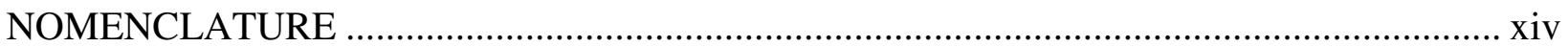

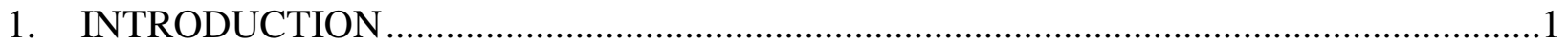

1.1. UAV Military Origins ...........................................................................

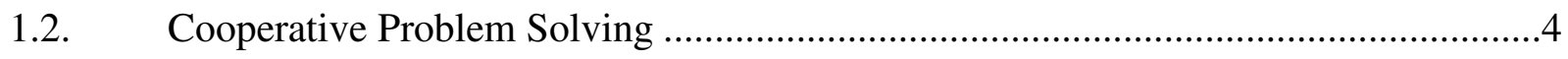

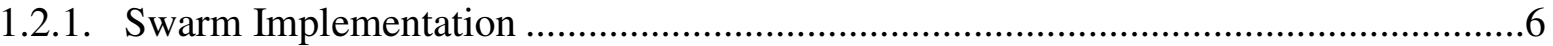

1.2.2. Parent-Child Implementation ..................................................................... 9

1.3. Civil Mission Applications for UAVs ............................................................... 10

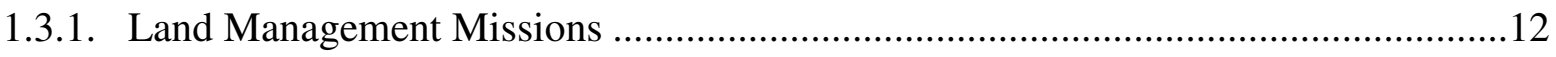

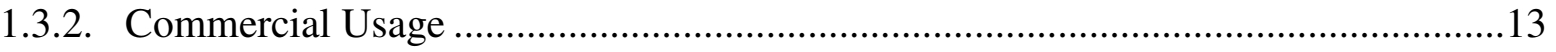

1.3.3. Earth Science Missions ........................................................................................... 14

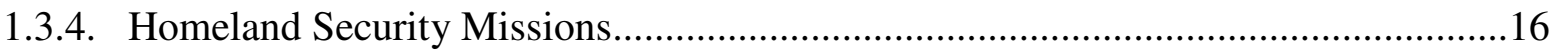

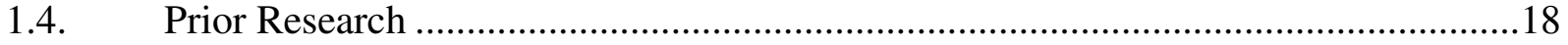

1.4.1. Initial Concept of Operations ......................................................................... 19

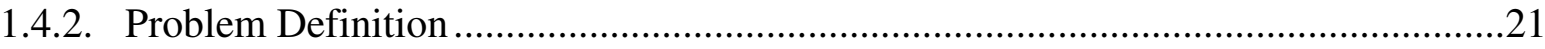

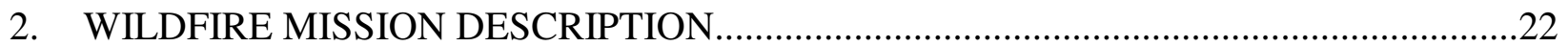

2.1. Mission Constraints ......................................................................................24

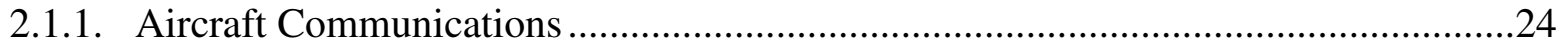

2.1.2. Civil Airspace Restrictions........................................................................26 


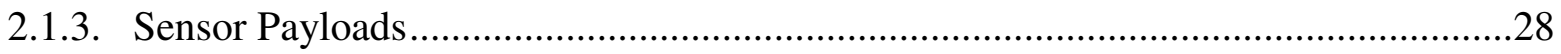

2.2. Mission Concept of Operations .............................................................................

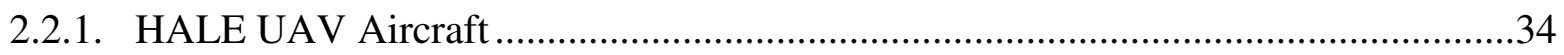

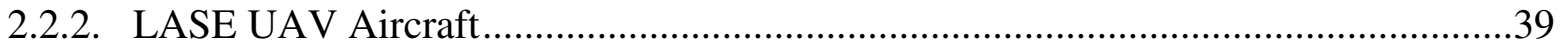

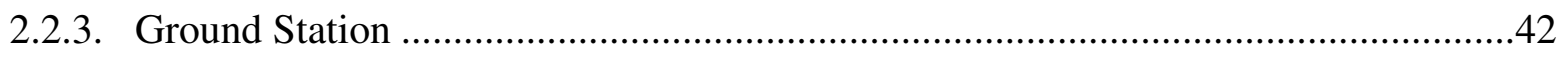

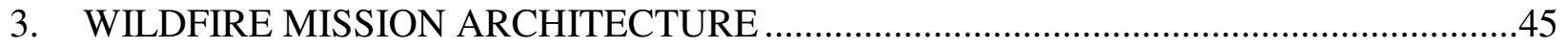

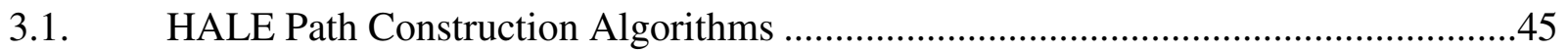

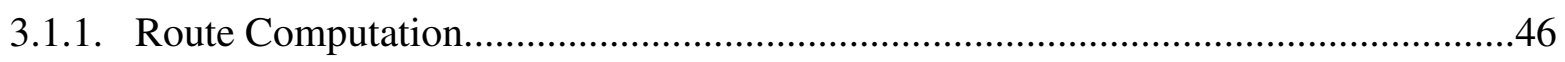

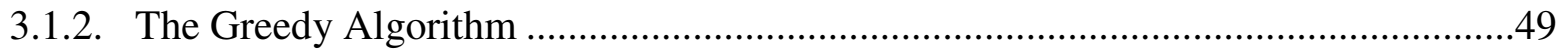

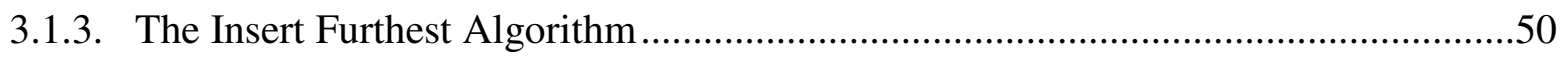

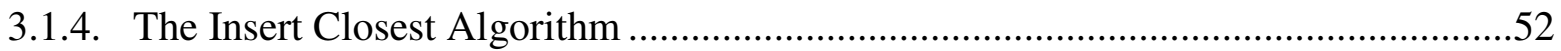

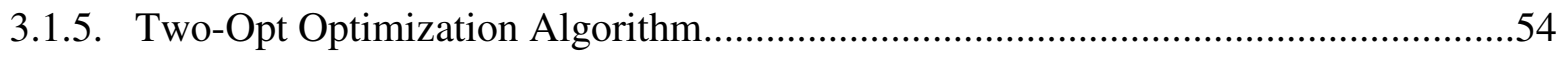

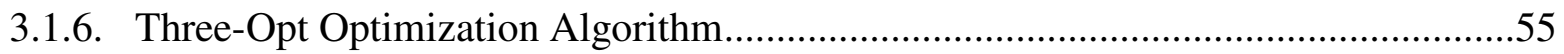

3.1.7. Algorithm Down-Selection ................................................................................5

3.2. HALE Architecture Search Algorithms ...........................................................61

3.2.1. HALE Sensor Configuration ................................................................................61

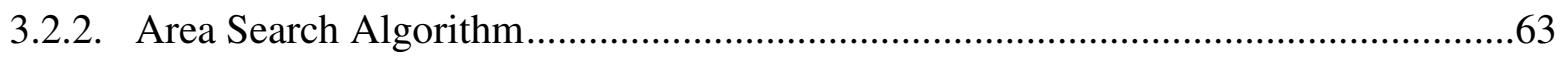

3.3. Aircraft and Ground Communications ................................................................67

3.3.1. Synchronous versus Asynchronous Communication ................................................68

3.3.2. Message vs. Object Oriented Communication ...........................................................

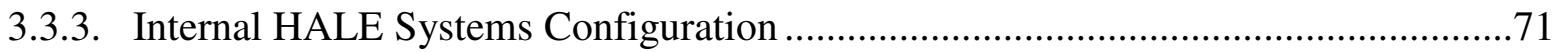

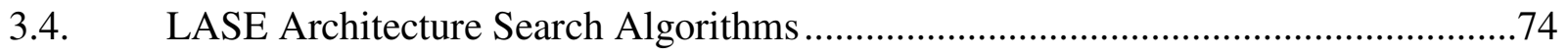

3.4.1. LASE Perimeter Algorithm Modes Overview ...........................................................75 


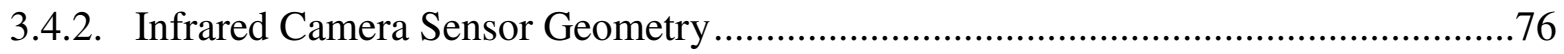

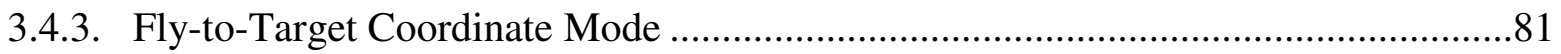

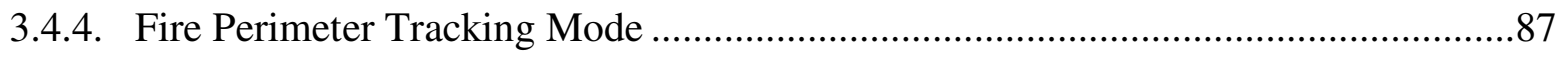

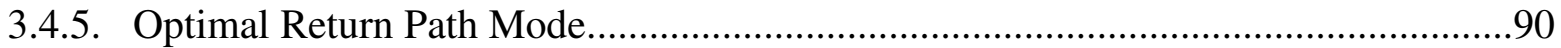

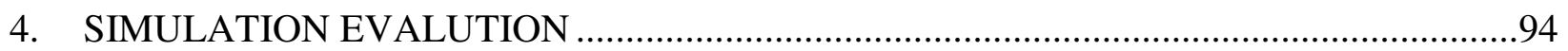

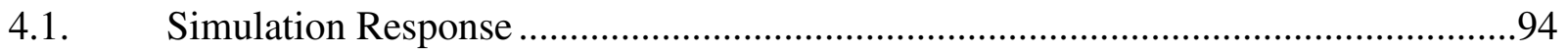

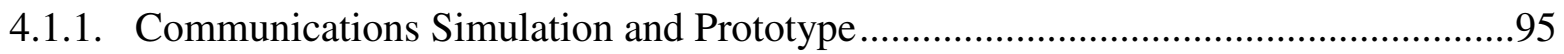

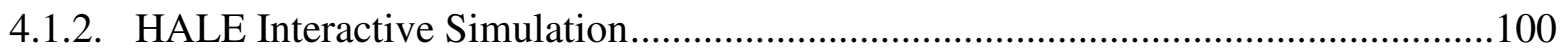

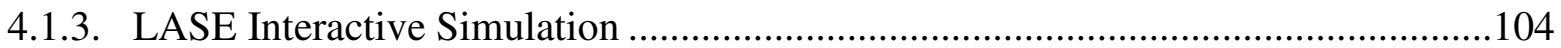

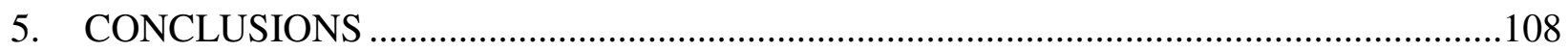

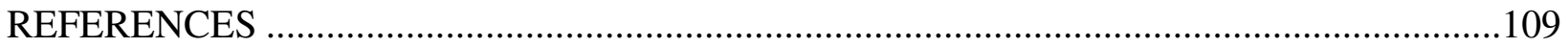

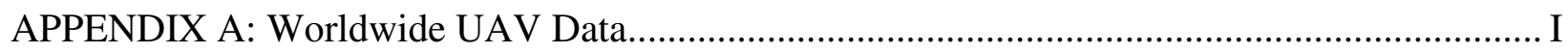

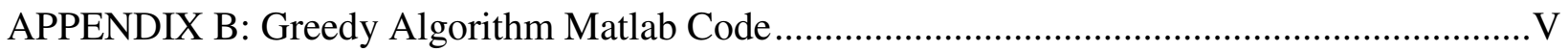

APPENDIX C: Insert Furthest Algorithm Matlab Code …………………………..................... VII

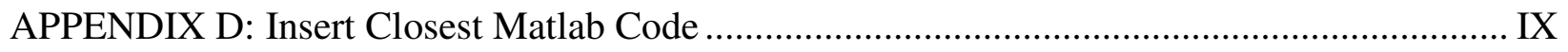

APPENDIX E: 2-Opt Algorithm Matlab Code ………………………………….................... XI

APPENDIX F: 3-Opt Algorithm Matlab Code.........................................................................II

APPENDIX G: LASE Matlab Simulation Code ……………………………....................... XVI

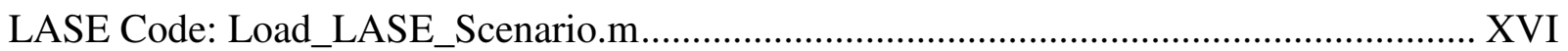

LASE Code: Model_Aircraft.m......................................................................................

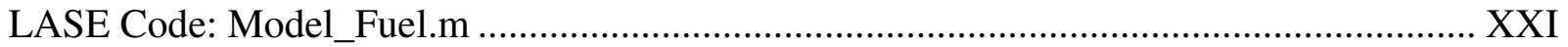

LASE Code: Model_Modes.m.............................................................................................XXIII

LASE Code: Model_Sensor.m...................................................................................XVIII 
LASE Code: Model_Return.m.................................................................................XXXI

LASE Code: Create_Perim.m.....................................................................................XXXIV

APPENDIX H: HALE Matlab Simulation Code .........................................................XXXI

HALE Code: Load_HALE_Scenario.m .............................................................XXXI

HALE Code: Model_Interface.m........................................................................XXXIX

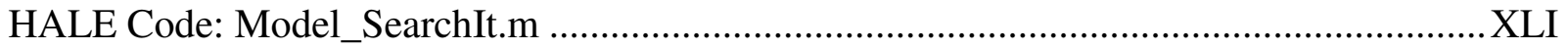




\section{LIST OF TABLES}

Table Name

Table 1: Sensor Types Available For Fire Detection and Response ${ }^{21}$

Table 2: Cost Matrix for Traveling Salesman Problem

Table 3: Computation Results for Sample 15 Node TSP Problem

Table 4: TSP Solution Data for 20 Nodes and 20 Simulations. 58

Table 5: TSP Solution Data for 50 Nodes and 20 Simulations. 59

Table 6: TSP Solution Data for 100 Nodes and 20 Simulations................................................... 59

Table 7: HALE Simulation Code Module Descriptions ……………………………................... 96

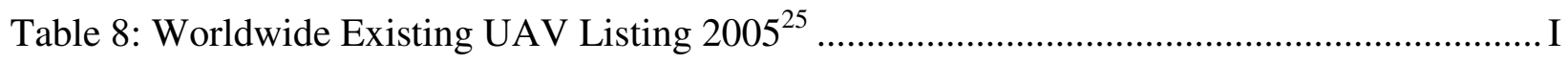




\section{LIST OF FIGURES}

Figure 1: DOD Annual and Projected Funding Profile for Unmanned Systems ${ }^{3}$........................... 2

Figure 2: Sheriden and Verplank Levels of Automation $(1978)^{4}$................................................. 3

Figure 3: Basic UAV Swarming Architecture Implementation................................................... 6

Figure 4: Department of Defense Plan for UAV Autonomy ${ }^{3}$....................................................... 7

Figure 5: Basic Parent-Child Architecture Implementation ....................................................... 9

Figure 6: Classification of Potential United States Civil UAV Markets ${ }^{1}$...................................... 11

Figure 7: Images of Yamaha RMAX UAV used for crop-dusting in Japan.................................. 13

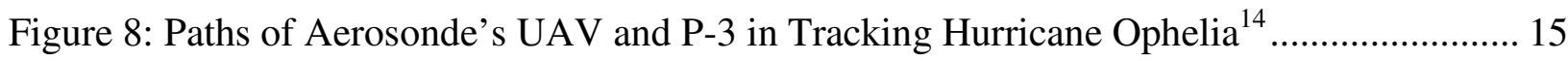

Figure 9: Mexico - U.S. Border with Temporary No-Fly Restrictions ${ }^{15}$...................................... 18

Figure 10: Technology Development Strategy for NASA WRAP Project ${ }^{17}$................................. 19

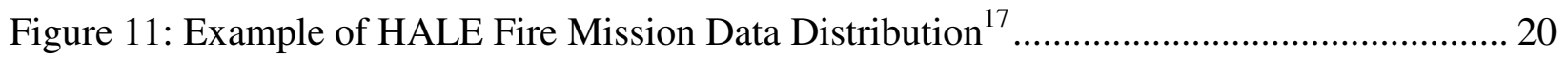

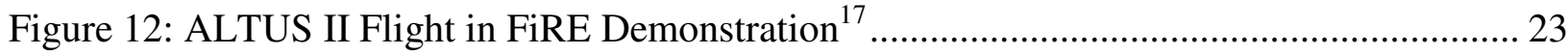

Figure 13: Current Commercial Aircraft Flight Tracks over the United States ${ }^{24}$......................... 26

Figure 14: ALTAIR-Western States Fire Mission Outline ${ }^{17}$ …….................................................. 27

Figure 15: UAV Payload versus Gross Weight Trend from UAV Data ${ }^{25}$.................................... 30

Figure 16: Collaborative Decision Environment Displaying Live Wildfire Data ${ }^{20}$..................... 32

Figure 17: Proposed HALE and LASE UAV Wildfire Architecture ………………...................... 34

Figure 18: Common High Altitude UAV Ascension Path to Cruise Altitude ${ }^{24}$............................. 36

Figure 19: Multi-Spectral Imagery Taken During Paradise Fire ${ }^{17}$ _......................................... 37

Figure 20: Sample Traveling Salesman Problem with 15 Nodes ................................................. 47 
Figure 21: Greedy Algorithm with No Optimization 49

Figure 22: Insert Furthest Algorithm with No Optimization .............................................. 51

Figure 23: Insert Closest Algorithm with No Optimization ............................................. 53

Figure 24: Two-Opt Optimization Algorithm with Random Initial Tour................................. 54

Figure 25: Three-Opt Optimization Algorithm with Random Initial Tour.............................. 56

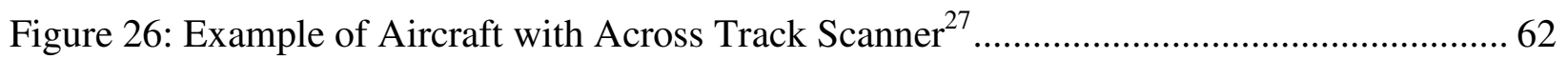

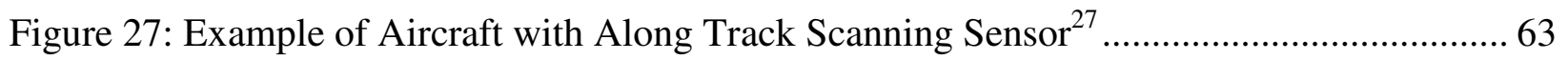

Figure 28: Example of Defined Wildfire Search Location ................................................... 64

Figure 29: Region with Rectangular Bounding Box for Search Pattern................................. 65

Figure 30: Example Search Pattern for Defined Wildfire Region ........................................ 66

Figure 31: Main Ground Station to HALE UAV Communications .......................................... 72

Figure 32: HALE Internal Information Flow Schematic ................................................ 73

Figure 33: LASE Mission Mode Logic Transitions ....................................................... 76

Figure 34: Minimum Aircraft Sensor Swath Radius Dimensions ........................................ 77

Figure 35: Minimum Sensor Field of View Dimensions ................................................... 79

Figure 36: Aircraft Forward Looking IR Camera Sensor Model ......................................... 80

Figure 37: Aircraft Maneuvering to Follow Fire Perimeter.................................................. 89

Figure 38: LASE Aircraft Return Path Algorithm Objectives............................................. 90

Figure 39: DOMS Middleware Architecture for the AuRA Simulation.................................. 97

Figure 40: Aircraft Progressing Toward Search Area with a Wildfire ................................... 98

Figure 41: Aircraft Locating Wildfire and Proceeding to Next Target ................................... 99

Figure 42: User Selection of HALE UAV Coordinates for Search Regions ........................... 101

Figure 43: HALE UAV with Optimized Route through Each Search Region ......................... 102 
Figure 44: HALE UAV with Linear Search Pattern Based on Optimal Routing

Figure 45: Selection of LASE Coordinates and Aircraft Tracking Response ...... 105

Figure 46: Completed LASE Simulation with Fire Perimeter Tracking 106

Figure 47: Completed LASE Simulation with No Wildfire Located 107 


\section{NOMENCLATURE}

\begin{tabular}{cl} 
Name & Description \\
\hline ATC & Air Traffic Control \\
AURA & Autonomous Robust Avionics Project \\
COTS & Commercial Off the Shelf \\
DOD & Department of Defense \\
DOMS & Distributed Open Messaging System \\
EGI & Embedded GPS/INS \\
FAA & Federal Aviation Administration \\
FiRE & First Response Experiment \\
GPS & Global Positioning System \\
GUI & Graphical User Interface \\
HALE & High Altitude Long Endurance \\
IFOV & Instantaneous Field of View \\
IMM & Intelligent Mission Management \\
IMU & Inertial Measurement Unit \\
INS & Inertial Navigation System \\
IR & Infrared \\
ITAR & International Traffic in Arms Regulations \\
LASE & Low Altitude Short Endurance \\
LEO & Low Earth Orbit \\
LiDAR & Light Detection and Ranging \\
LOS & Line of Sight \\
NAS & National Airspace System \\
NASA & National Aeronautics and Space Administration \\
NIFC & National Interagency Fire Center \\
OTH & Over the Horizon \\
RF & Radio Frequency \\
ROV & Remotely Operated Vehicle \\
RSAC & Remote Sensing Applications Center \\
SAR & Synthetic Aperture Radar \\
TCP & Transmission Control Protocol \\
TFR & Temporary Flight Restriction \\
TSP & Traveling Salesman Problem \\
UAV & Unmanned Aerial Vehicle \\
USFS & United States Forest Service \\
WHF & Very High Frequency \\
Wildfire Research and Application Partnership \\
Wide Web \\
WAP
\end{tabular}


operate them. In order to guide this process and aid in their evolution, a roadmap for UAV development was established by the Department of Defense. ${ }^{2}$ Along with outlining existing systems and their military applications, this roadmap listed a series of key technological hurdles. After experimentation and development, the utility of UAVs in a combat environment was recognized and the desire to further UAVs beyond standard remote guidance was born. In order to achieve this aim, the Department of Defense set its sights towards pioneering aircraft autonomy. ${ }^{3}$

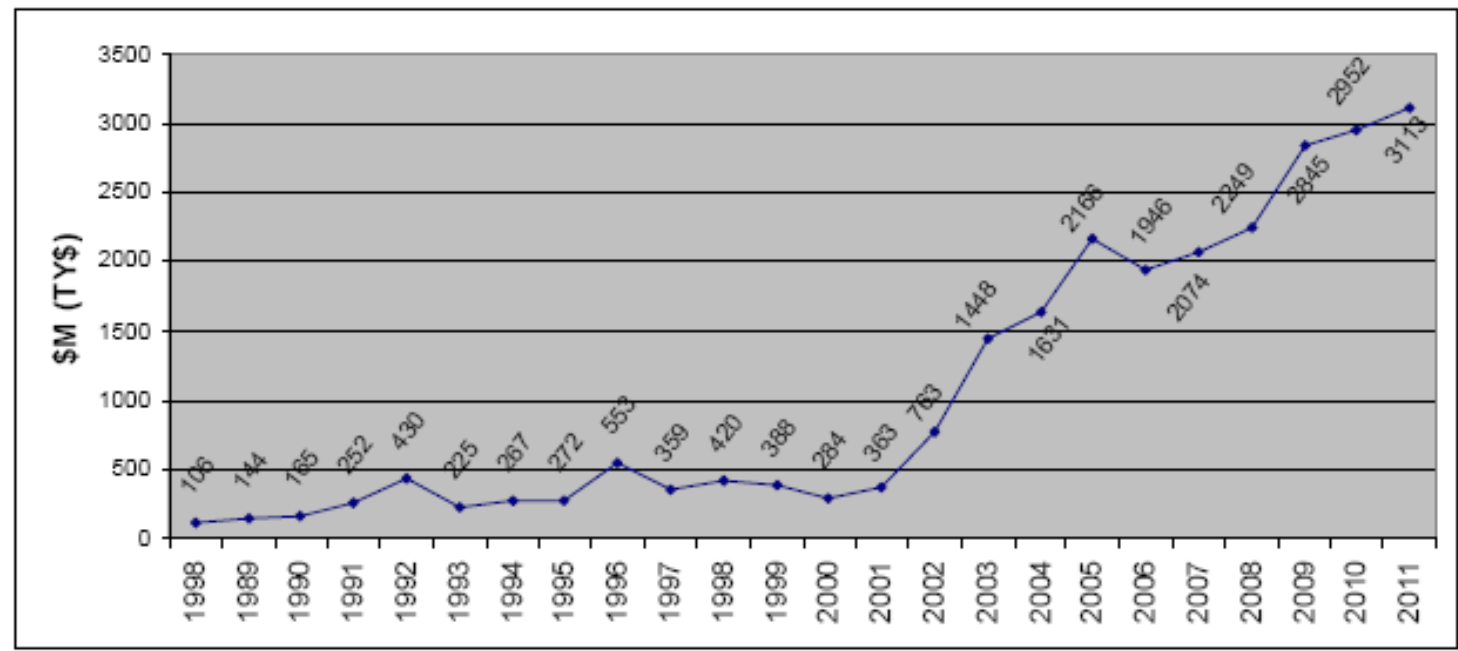

Figure 1: DOD Annual and Projected Funding Profile for Unmanned Systems ${ }^{3}$

UAV autonomy has come to represent a variety of aircraft capabilities ranging from health monitoring and contingency management to mission planning and self guidance. ${ }^{1}$ These capabilities, however, underlie the true measure of vehicle autonomy: the amount of human supervision required for mission completion. ${ }^{4}$ This can range from minimal autonomy, such as when the vehicle's decisions are approved remotely by a human operator, to extreme cases in which plans are executed while disregarding human interaction. As a result, the definitive representation of vehicle autonomy becomes the ability to reject human requests when the suggestions are not in the vehicle's best interest. ${ }^{5}$ A prime example is the ability for the aircraft 
to avoid destruction by determining, and subsequently avoiding, obvious examples of human error.

\begin{tabular}{|c|c|}
\hline $\begin{array}{l}\text { Automation } \\
\text { Level }\end{array}$ & Automation Description \\
\hline 1 & $\begin{array}{l}\text { The computer offers no assistance: human must take all decision and } \\
\text { actions. }\end{array}$ \\
\hline 2 & The computer offers a complete set of decision/action alternatives, or \\
\hline 3 & narrows the selection down to a few, or \\
\hline 4 & suggests one alternative, and \\
\hline 5 & executes that suggestion if the human approves, or \\
\hline 6 & allows the human a restricted time to veto before automatic execution, or \\
\hline 7 & executes automatically, then necessarily informs humans, and \\
\hline 8 & informs the human only if asked, or \\
\hline 9 & informs the human only if it, the computer, decides to. \\
\hline 10 & $\begin{array}{l}\text { The computer decides everything and acts autonomously, ignoring the } \\
\text { human. }\end{array}$ \\
\hline
\end{tabular}

Figure 2: Sheriden and Verplank Levels of Automation (1978) ${ }^{4}$

In efforts to further advance this concept of autonomy, research money has been provided not only for defining the decision-making algorithms that drive these aircraft, but also for enabling a vehicle to collaborate with additional UAVs in completing certain tasks. ${ }^{3}$ The rationale for this approach stems from the fact that as UAVs acquire more prominent roles; their need to interact with both new and existing mission resources becomes critical. Additionally, as the number of UAVs increases, so does the need for these aircraft to communicate and rely on one another for mission completion. ${ }^{3}$

One resolution for this anticipated need has been to research the concept of distributed systems and robotic group behavior. Distributed systems, a form of parallel computing, provide a means of dividing information among all feasible resources to determine a solution. While this aids in the dispersal of information, the operation remains incomplete unless a framework is developed 
as to how each resource makes its decision. Group behavior provides those rules under which each resource can process the information toward a feasible solution. In essence, distributed systems provide the means of disseminating information while group behavior allows the resources to determine a course of action. The next endeavor becomes establishing the concept of teamwork and cooperation into the aircraft.

\subsection{Cooperative Problem Solving}

While many communications, payload, and flight controls have already matured as technologies, the move toward full vehicle autonomy has developed at a much slower pace. The limited progress is due to the preference for remote human operation. ${ }^{1}$ This predilection is based on the idea that human operators are superior at maintaining control of UAV resources and preventing accidents or operational errors. The drawback of maintaining this mentality is that the cost of maintaining multiple human operators and ground stations grows exponentially as the number of UAVs increases. ${ }^{6}$ Moreover, increasing the command complexity, miscommunication, and number of operators will result in the type of scenarios that cause human control errors. This can be prevented through the realization that machines excel at the mundane and tedious tasks presented by UAV operation. It is only with a de-emphasis of human control and application of research money toward UAV planning algorithms that the era of cooperative UAV problem solving will begin.

Cooperative multi-agent planning has long been the domain of artificial intelligence and

robotics. ${ }^{5}$ Only recently have these technologies been applied to an aircraft platform to execute and assess plans in real-time with consideration for vehicle constraints. One of main the 
obstacles faced by UAVs are that executed plans must be flexible in time and space. This includes the ability to respond to unpredictable changes in the environment, alter plans rapidly, with tight coordination, and do so within vehicle constraints. ${ }^{7}$

For these applications, multi-agent planning can be defined as: planning by multiple agents for a common goal, agents coordinating plans and merging the plans of others, or agents refining their own plans while negotiating over resources. This also involves the execution of such plans in real-time while determining contingencies. ${ }^{8}$ While the particular solutions to each problem differ within the mission, two general approaches can be identified for controlling multiple agents: swarming (distributed command) and parent-child (centralized command) architectures. ${ }^{7}$ Each of these architectures possesses inherent strengths and weaknesses but can be applied singly or combined for a wide range of applications. 


\subsubsection{Swarm Implementation}

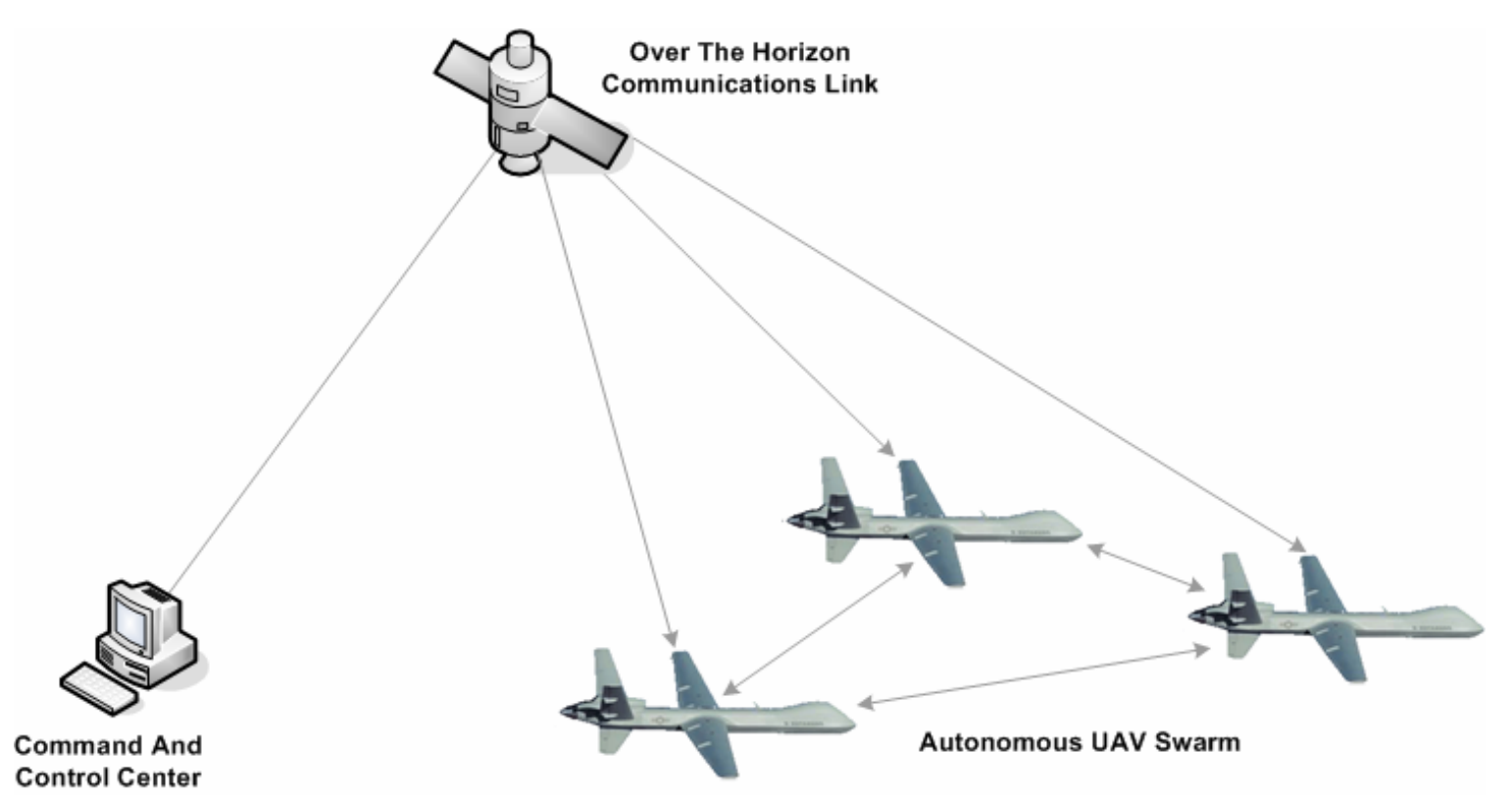

Figure 3: Basic UAV Swarming Architecture Implementation

Identified as the ultimate goal in the Department of Defense issued Unmanned Aircraft Systems Roadmap, the aim of this ideology is to use autonomous swarms of cooperative UAVs to fulfill a particular task. ${ }^{3}$ Such architecture is useful addressing hazardous scenarios during which larger numbers of UAVs can be used to eliminate a single point of failure. Additionally, multiple differing UAVs can be used to address a single mission and re-plan the task based on outcome uncertainty and the capabilities of each aircraft. The target selection is maintained under human control while completion is left to the agents, making this structure ideal for military applications. ${ }^{5}$ 


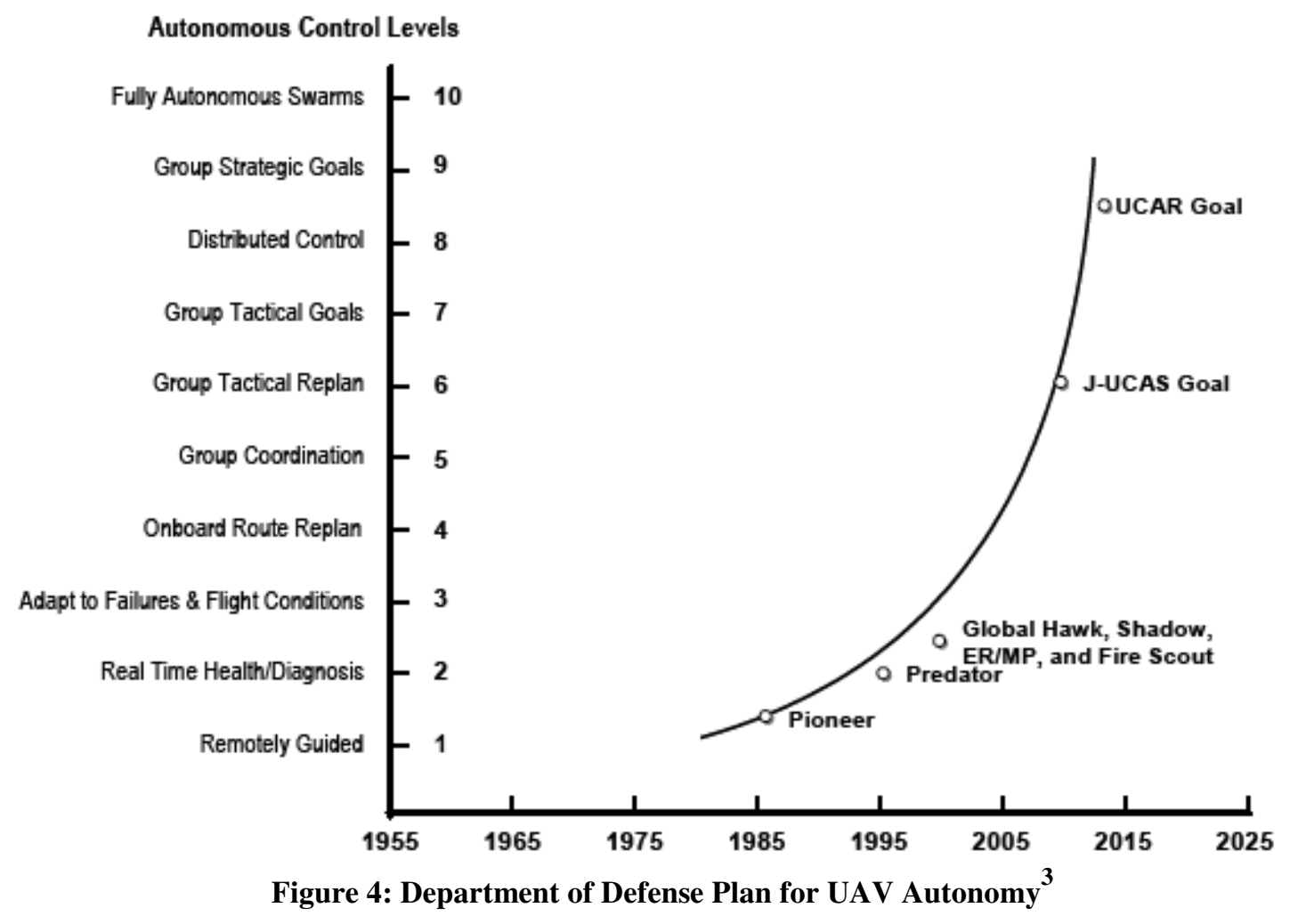

However, swarm implementations have their weak points. First and foremost, swarm logic is based on the concept of all vehicles working toward a single mission, built on a bidding procedure where UAVs must compete for a mission role based on their functional abilities. As expected, a high volume of communications traffic is generated as each UAV vies for suitability to complete tasks in the mission. The exchange occurs as each aircraft expresses interest or disinterest in the sub-task. Once the task has been awarded, the UAVs must then coordinate dynamically with one another. ${ }^{9}$ Because line of sight (LOS) communications are critical to this behavior, the methodology breaks down when the agents are dispersed over large regions. This occurs since satellite communication factors such as latency, periodicity, and bandwidth requirements make local communications becomes the only feasible option. Therefore, constant communication must be funneled through basic transmitters like VHF or RF with limited range. The methodology then revolves around the concept of the overall task being handed down from a 
human operator. This outlines a clear and concise objective where the UAVs can vie based on aptitude, health, or location and cost it against a known maneuver or quantity. As a result, a collective UAV swarm implementation does not scale well to problems with vague mission goals or where localized swarms must react to events over expansive regions, which occurs due to large dispersion of swarms neutralizing the advantage of being able to send the most suitable UAV. Additionally, sending multiple UAVs on purely reactive reconnaissance missions is wasteful since it cannot be tuned to the mission requirements.

An instance where democratic swarm behavior would be encapsulated in a mission is where three similar UAVs are sent to destroy a human designated target. Once the directive is sent to the aircraft, the three fly to investigate the situation. On approach to the target, the three aircraft cross communicate in order to determine the attack sequence and the most suitable UAV for each portion of the mission. Finally, the plan is executed while changing in real-time as the aircraft communicate and adapt to the situation. 


\subsubsection{Parent-Child Implementation}

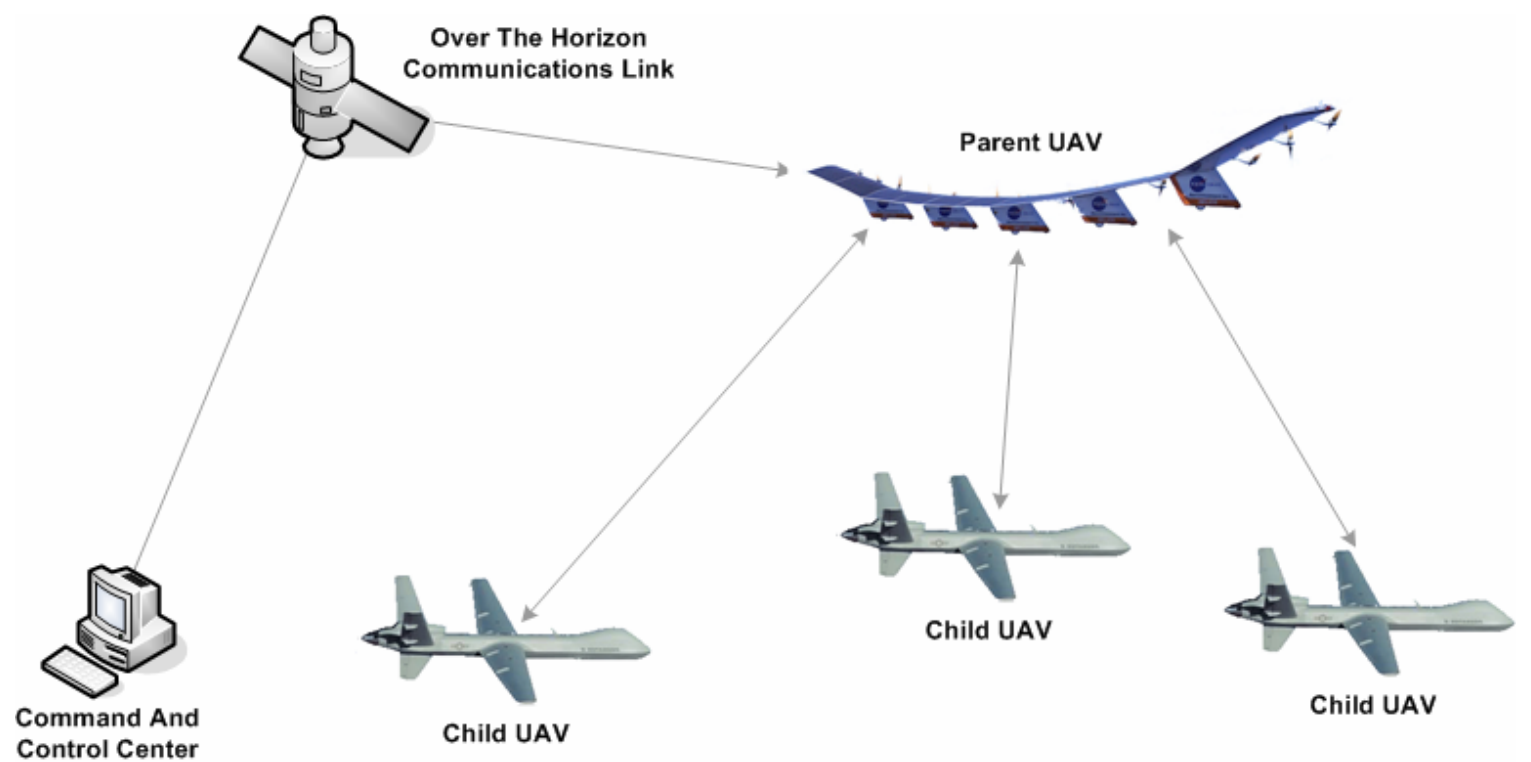

Figure 5: Basic Parent-Child Architecture Implementation

The centralized, or parent-child architecture, establishes that while each UAV retains its autonomy, an overseer UAV is appointed so it can assign sub-tasks to agents responsible for their completion. ${ }^{4}$ Unlike swarming, the master UAV knows the overall goal and uses the capabilities of the agents under its control to carry out the chosen mission. Additionally, the architecture is most appropriate for surveillance tasks where the objective is observation and reaction. ${ }^{4}$ This is because response with multiple assets is ill-suited for these vague observation missions where a single capable UAV can be sent. Another reason for the architecture's advantage in surveillance missions requiring response is because multiple assets are left to idle until the event occurs.

It is critical to remember this architecture is subject to limitations and especially those arising from communications. Since a master UAV is utilized for much of the active decision making, this can serve as a single point of failure in tasking the subordinates. Moreover, a single aircraft 
is in charge of agents, which may lead to a computational and communication strain being placed on the aircraft in addition to its nominal operating tasks. ${ }^{6}$ Most critically, with the master UAV overseeing the task, the aircraft is essentially completing a task of human operation. This requires a high level of autonomy and trust in the decision-making process, resulting in military applications finding this architecture difficult as it acts in lieu of human supervision.

For parent-child architectures, the command structure acts like a hierarchy with directives flowing through a chain of command. An example of this instance would be an advanced aircraft tasked with patrolling a particular region for potential threats. If an unidentified target is

detected, the aircraft programs a drop ship with the required information and then releases the drone. The drone then continues the mission by closely examining the target and reporting the information back to the master aircraft for relay back to the human operators.

\subsection{Civil Mission Applications for UAVs}

While UAVs are slowly becoming incorporated within the civil sector, their applications are still being guided by their original military development attitude where man has been deemed the limitation. ${ }^{3}$ This is illustrated by the military slogan of "dull, dirty, and dangerous" in describing missions ideal for UAV operations. The "dull" missions are characterized by the basic limitation of fatigue. Although many understand this to be found within lengthy missions where human occupants are incapable of withstanding more than forty hours of operation, this can also encompass processing the immense amounts of data that can be generated by surveillance missions. The sampling of potentially hazardous materials or lethal environments has proven to 
be the main concern within "dirty" missions. "Dangerous" missions are defined when the complete loss of an aircraft is possible and a human life is an unnecessary risk. ${ }^{3}$

The above defined missions and current capabilities have led to multiple countries and international organizations expressing interest in operating UAVs. Locally, this has included American institutions, such as research centers, government agencies, and corporations, supported by the DOD's efforts to push development into the private sector sharing the research costs and development of new ideas. ${ }^{3}$ Figure 6 , below, contains a listing developed by a NASA study identifying parties interested in UAV technologies. With their own take on how vehicle autonomy can be incorporated into their responsibilities and products, many of these organizations have not only expressed interest, but outlined their mission requirements in a variety of conferences and studies.

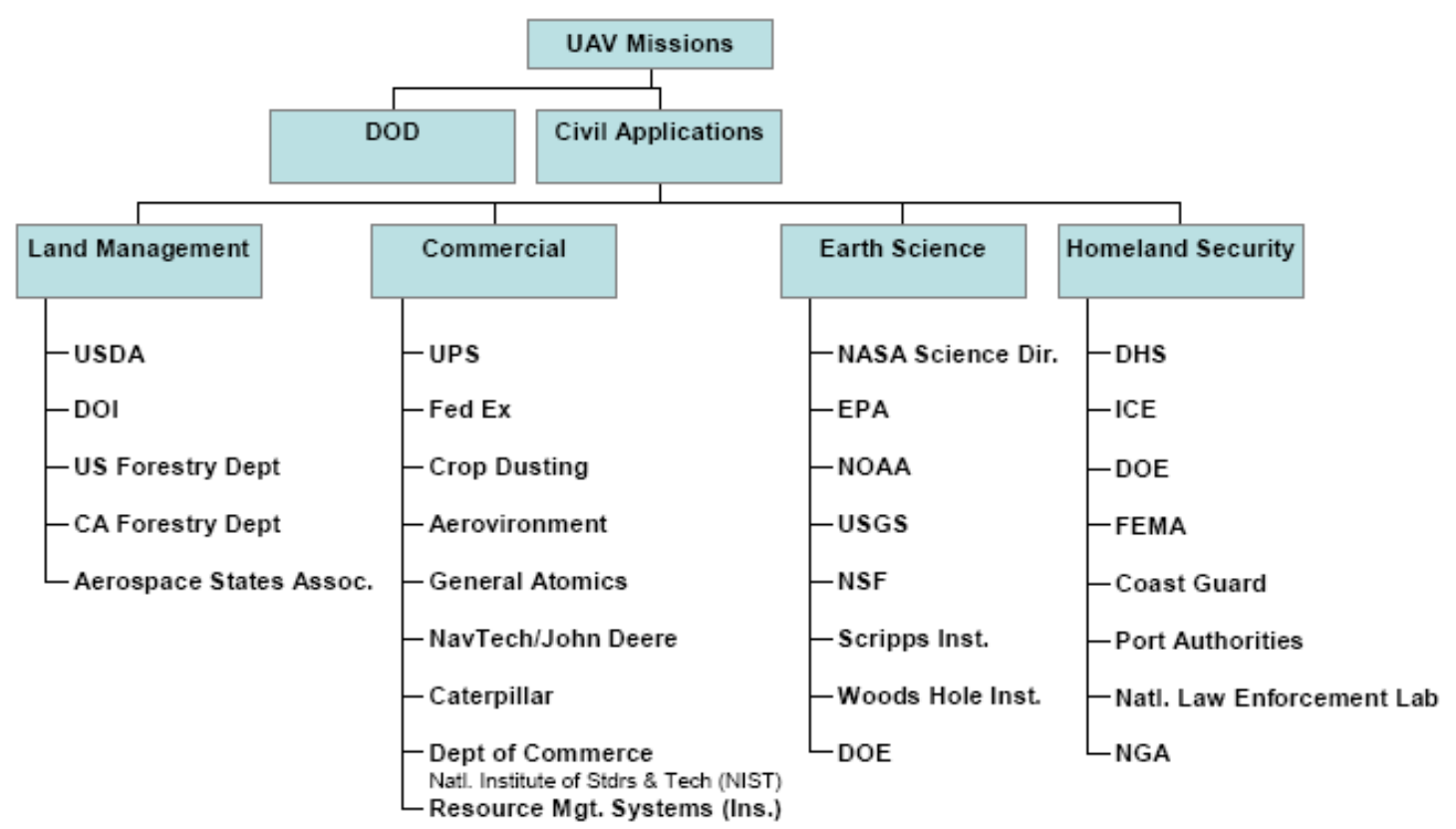

Figure 6: Classification of Potential United States Civil UAV Markets ${ }^{1}$

As shown above, those interested in civil UAVs are separated into four categories, each with their own distinct need. ${ }^{3}$ The first category, land management, is comprised of governmental 
agencies devoted to determining and monitoring environmental changes. The second comprises commercial agencies that desire to invest in UAV development as a marketable product for distribution and support of current activities. The third, earth sciences, includes research institutes seeking to monitor and evaluate global changes. Finally, homeland security desires support in performing a variety of disaster and response missions.

\subsubsection{Land Management Missions}

UAVs are suitable for missions involving land management and for monitoring natural disasters, due to their ability to perform long duration reconnaissance tasks. This eliminates the need to risk the lives of human pilots while operating under hazardous conditions. The U.S. Forest Service (USFS) and the Department of Agriculture are most interested parties in this particular application.

The USFS finds their primary concern as the detection and response to fires. Potential missions include providing communications for firefighters on the ground, mapping fire perimeters, damage assessment, and delivering fire retardant to the fires themselves. In addition to fire concerns, the USFS also sees the potential need for wild animal tracking and plant growth

monitoring. ${ }^{10}$ In a similar manner, the Department of Agriculture is researching the possibility of designing UAVs with the ability to monitor agricultural practices such as grazing patterns, water management, soil conditions, and crop status. ${ }^{11}$ 


\subsubsection{Commercial Usage}

A variety of companies have expressed interest in operating UAVs to support their operations. Applications in agriculture, comprised mostly of lengthy or routine monitoring missions, have proven the most viable route for introducing UAVs into the commercial sector. Many of these areas mirror land management missions but also include active roles, such as crop dusting, shown in Figure $7 .{ }^{12}$ Additionally, the capability of UAVs to fly lengthy distances autonomously has appealed to delivery companies such as United Parcel Service (UPS) and FedEx to support their cargo delivery infrastructure. While many of these possibilities generate active discussions, the unfortunate reality remains - UAVs have much improvement before entering the commercial sector due to restrictions governing civil airspace and export controls governing UAV technology.
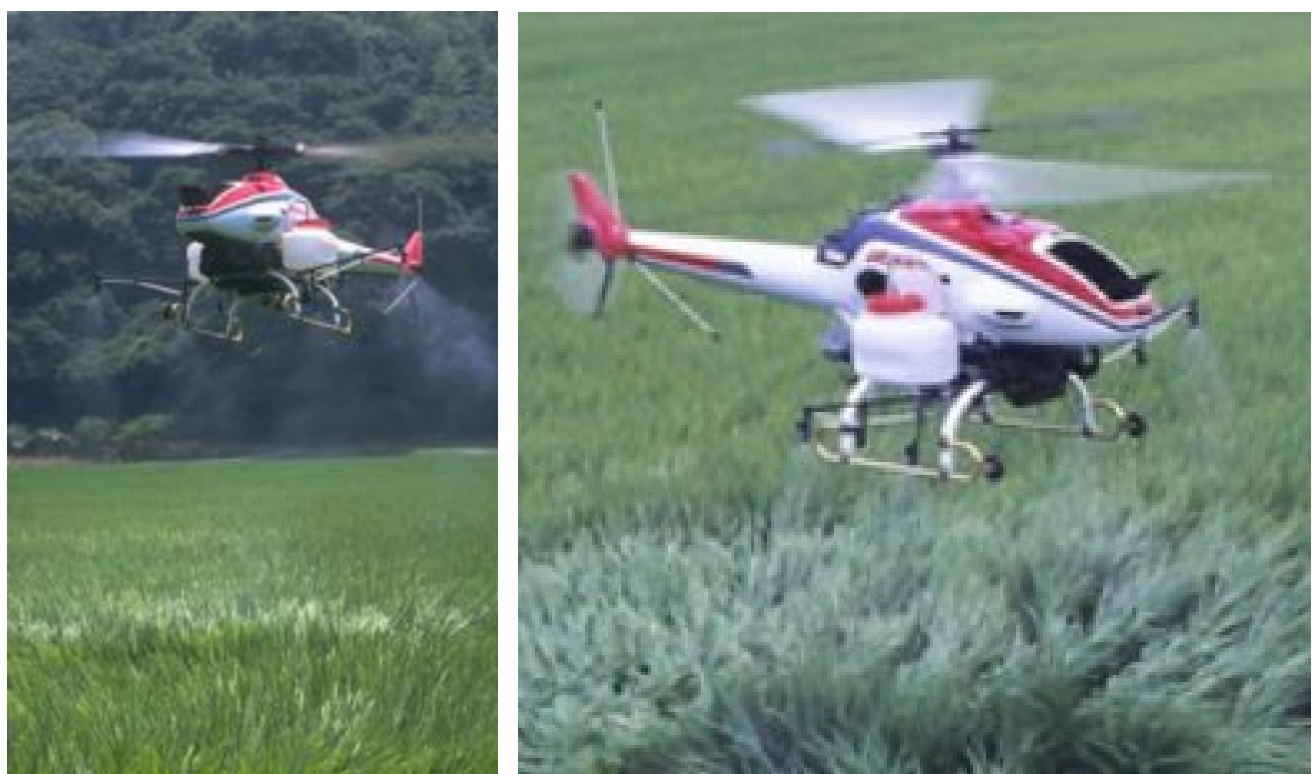

Figure 7: Images of Yamaha RMAX UAV used for crop-dusting in Japan

The first issue, access to national airspace (NAS), is a priority since a myriad of restrictions governs autonomous aircraft when a pilot is not present. ${ }^{12}$ These restrictions include developing see-and-avoid technologies to detect oncoming aircraft and avoid a collision, since aircraft are 
not required to carry transponders while in all locations of the NAS. Even when carrying transponders, the trajectory of the aircraft cannot be directly determined to ensure collision avoidance. Allowing UAVs as trusted users of the national airspace would allow rapid response and real-time tasking, or develop event driven tasking as opposed to pre-planned routes. ${ }^{11}$ Collaborative efforts between the Federal Aviation Administration (FAA) and a consortium of aircraft manufacturers and government agencies are underway to allow UAVs to fly reliably and routinely through the NAS. ${ }^{13}$

Second, export controls are a looming concern for commercial involvement, especially since many corporations have investments extending beyond American borders. International Traffic in Arms Regulations (ITAR) restrictions become a regulating factor regarding specific products leaving the United States, which is particularly true for limiting various sensor payloads, aircraft platforms, and cooperation with foreign entities in developing new UAV technologies. ${ }^{1}$ Such an obstacle may be overcome by importing foreign UAV platforms and sensors, since they are not regulated under governmental arms restrictions. As UAVs are developed in Europe and Asia, their potential for commercial use is more viable. ${ }^{1}$ Unfortunately, until UAVs are more commonplace in the world and trusted to operate unhindered alongside standard air traffic, progress towards using UAVs in American airspace will be hindered.

\subsubsection{Earth Science Missions}

Among the most diverse applications for UAVs are earth science missions studying environmental change. Whether applied to observation of earth, vegetation, coastal, oceanic, atmospheric, or arctic patterns, these types of missions are composed of periodic reconnaissance 
and tedious data collection. UAVs are ideal for these missions since the uninhabited aircraft can cover vast areas and collect information using remote sensing and are able to do so even under daunting environmental conditions.

Data collection missions illustrating this advantage can vary from earth measurements, soil sampling, vegetation composition and topical mapping to measuring ozone chemistry, ambient temperatures, local winds, and pressure at various locations. This can include measuring oxygen and carbon dioxide fluxes on land or sea with detailed resolution. These missions also can be especially useful in charting environmental changes over long durations and extracting global patterns. These same monitoring abilities can also be applied for arctic missions such as examining ice sheet and glacier movements. ${ }^{11}$ A prime example of a UAV utilized for this purpose can be found below in Figure 8. This depicts the Aerosonde's first successful flight of a UAV to track a hurricane on September 16, 2005. ${ }^{14}$

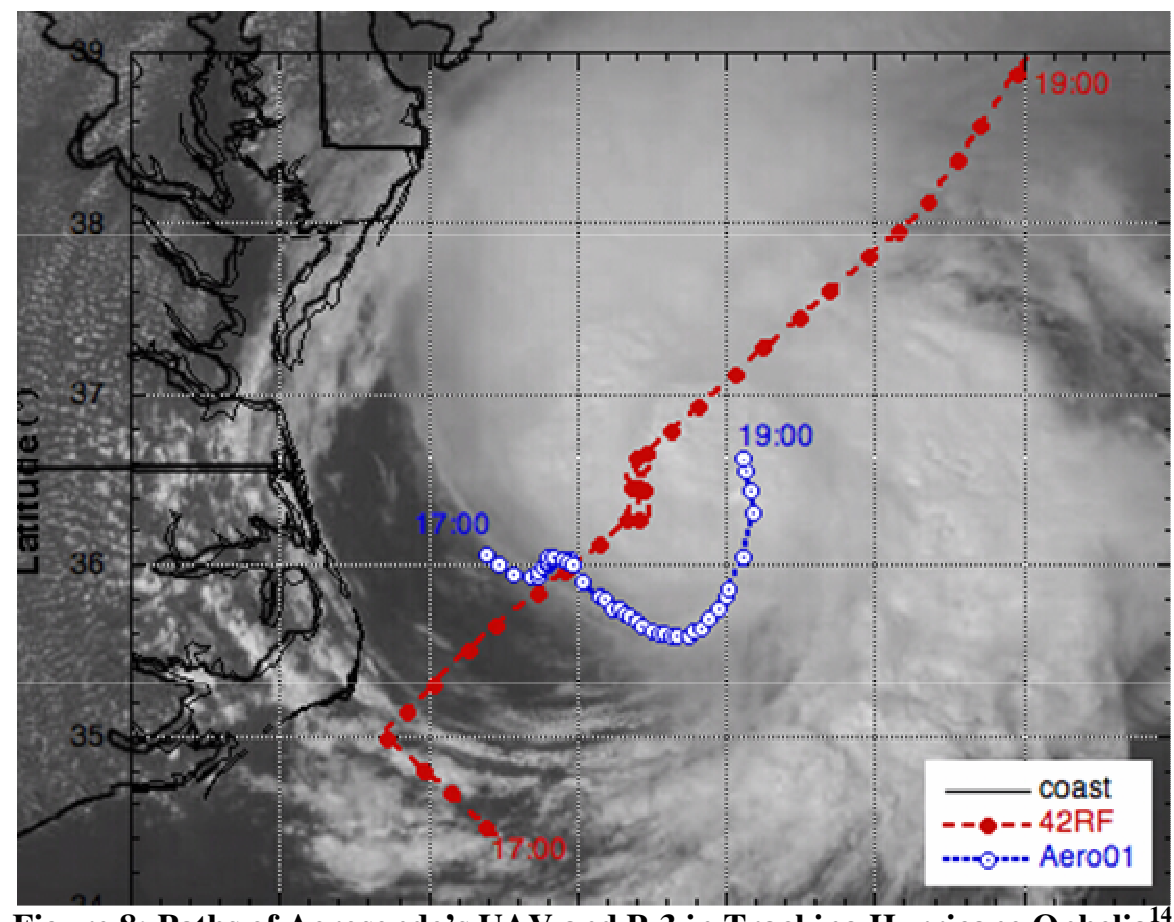

Figure 8: Paths of Aerosonde's UAV and P-3 in Tracking Hurricane Ophelia ${ }^{14}$ 
These earth science geared applications are especially useful to government agencies responsible for studying and predicting trends such as hurricanes, global warming, sea currents, and natural disasters. Multiple countries including Israel, Canada, Australia, and multiple European nations are investigating these research possibilities. ${ }^{1}$

\subsubsection{Homeland Security Missions}

Since the events of September 11, 2001 the concern for border security both on land and sea has grown dramatically. The recognition that UAVs are particularly suited for long duration reconnaissance missions, including border enforcement and tracking over large regions is being utilized in an effort to support the Department of Homeland Security. As a result of these advantages, two particular departments, the United States Coast Guard and the United States Border Patrol have shown interest in the abilities of UAVs.

The United States Coast Guard has stated that UAVs are an optimal choice for maritime surveillance. This surveillance includes missions where UAVs are utilized to patrol large regions in order to detect, monitor and track vessels that pose a risk. Their capacity for aerial monitoring is particularly useful against drug runners utilizing fast boats in an attempt to outrun the Coast Guard fleet. Also, a UAV's ability to perform mundane tasks makes it an ideal candidate for surveillance of standard maritime traffic. A large number of manned planes cannot be committed for this task and large fleets of UAVs can be constantly deployed with only minimal mission management capability. 
The second of these departments, the United States Border Patrol is expressing keen interest in a wide array of sensor packages including infrared, LIDAR, and SAR which UAVs can support as well as camera based surviellance. ${ }^{1}$ Equipped with this wide variety of sensors UAVs provide constant airborne coverage making them an ideal augmentation for ground forces. Proposed uses have also included tunnel detection, standoff tracking of located targets, communication nodes and vehicle identification which also allows a reduction of human risk where entities can be identified as friend or foe and/or armed or unarmed long before contact. ${ }^{11}$ Unfortunately, the Border Patrol must perform these tasks while operating in commercial and national airspace and, as a result of their immaturity as a technology, pose a threat to regular air traffic. ${ }^{14}$ As shown in Figure 9, a Temporary Flight Restriction (TFR) for general air traffic was established at the United States - Mexico border to allow for constant UAV operations. Having such a restriction in place, UAV operations are pushed into direct competition with general aviation, with future TFRs threatening to place a burden on airspace operations. ${ }^{14}$ An alternative is flying below general aviation traffic, but it comes as a heavy sacrifice to the high altitude vantage point that UAVs require for optical sensing. 


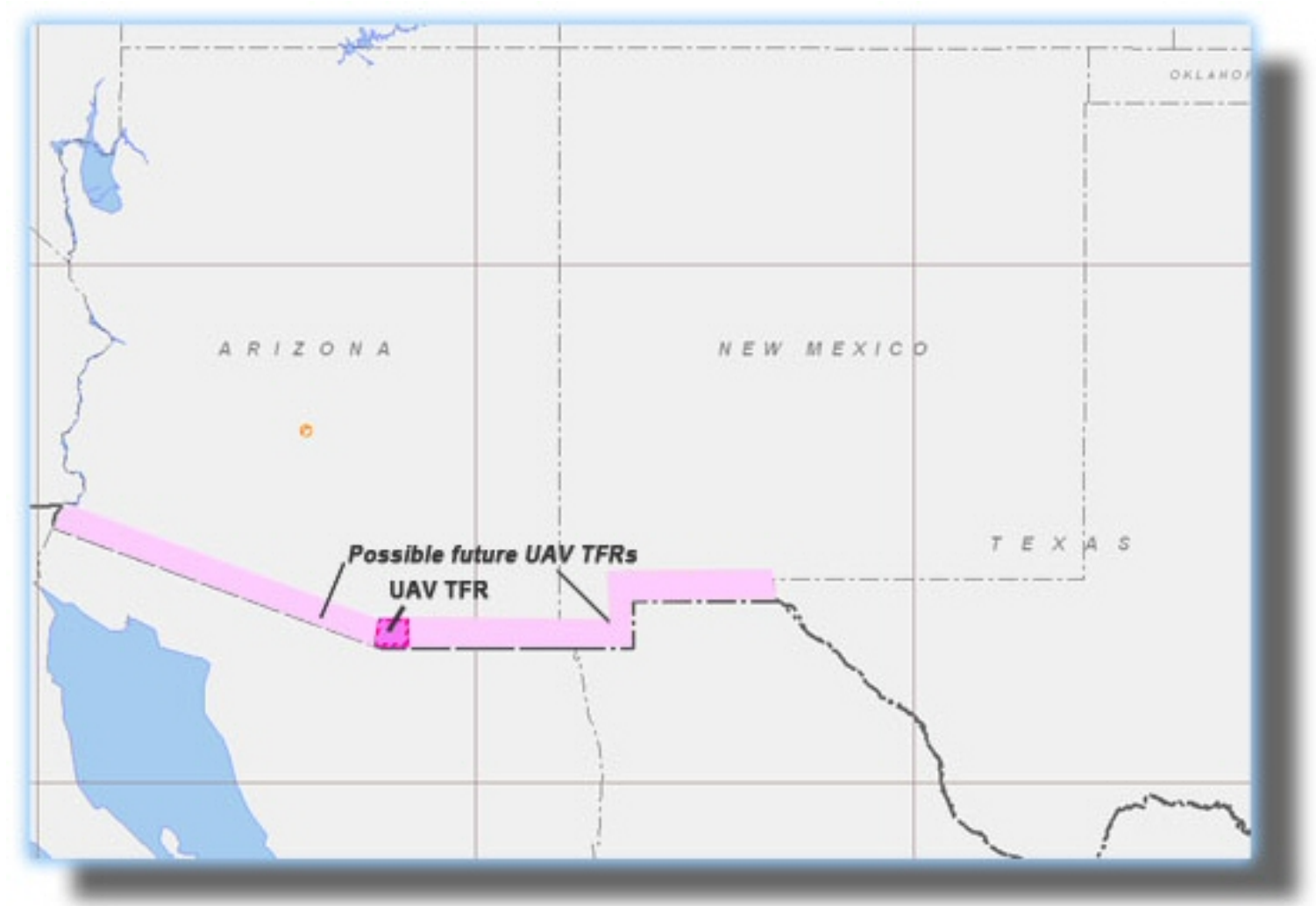

Figure 9: Mexico - U.S. Border with Temporary No-Fly Restrictions ${ }^{15}$

\subsection{Prior Research}

In 2005, NASA's Vehicle System Program was investigating potential applications for UAV operations. Of particular interest was a group designated under Intelligent Mission Management (IMM) for UAV autonomy. In the same timeframe, NASA and the United States Forest Service (USFS) were partnering to study UAVs for long duration monitoring of wildfires under the Wildfire Research and Application Partnership (WRAP) project, shown in Figure 10. Through this cooperative effort, the Intelligent Mission Management (IMM) sub-group joined the WRAP project to develop UAV autonomy and decision support systems for wildfire detection and response. ${ }^{16}$ 


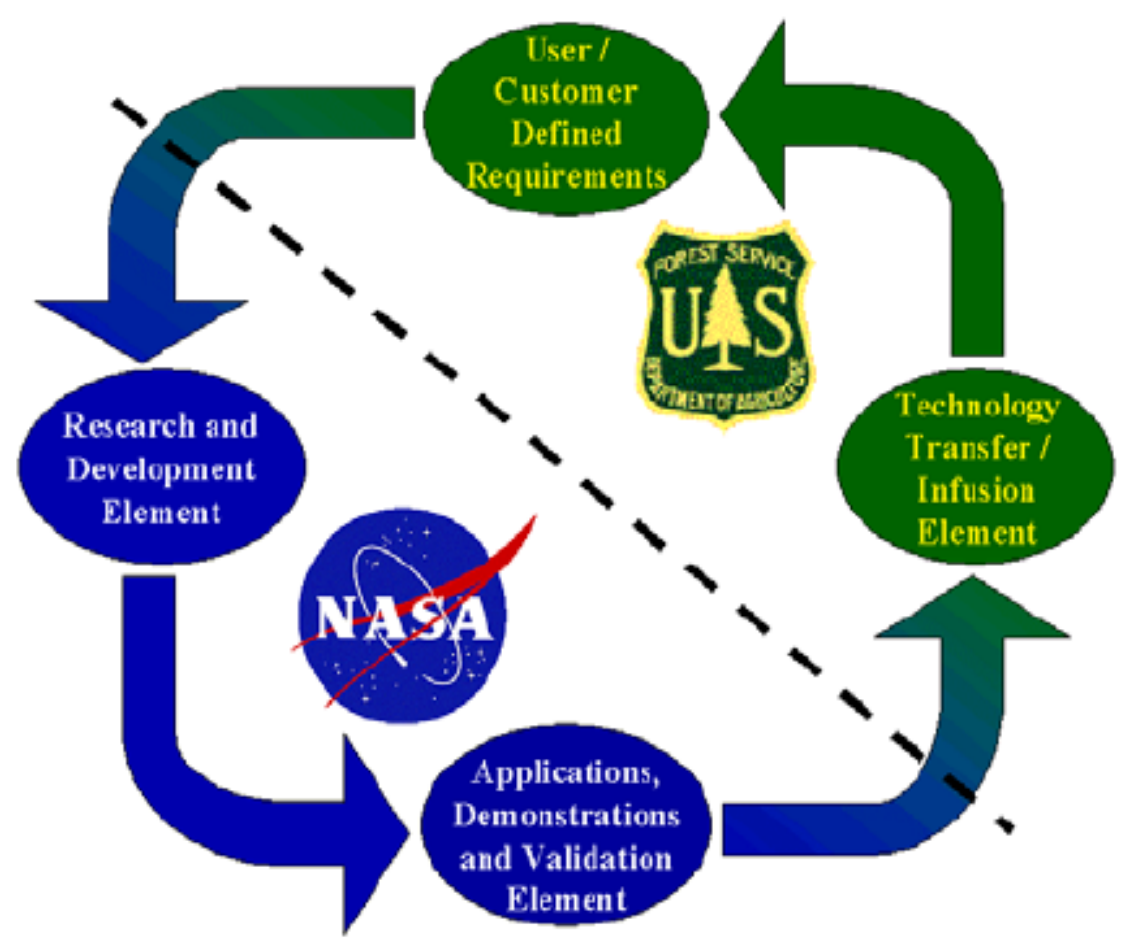

Figure 10: Technology Development Strategy for NASA WRAP Project ${ }^{17}$

\subsubsection{Initial Concept of Operations}

Under the WRAP partnership, a concept of operations was developed for a High Altitude Long Endurance (HALE) aircraft in determining wildfire response. ${ }^{18}$ As part of the concept of operations, a HALE UAV would receive information on potential wildfires through Moderate Resolution Imaging Spectroradiometer (MODIS) data provided via satellite or phoned in fire reports. The HALE UAV then proceeds to these locations and initiate search patterns for these potential fires that may or may not exist. ${ }^{17}$ The final data would then be transmitted back to the main command center using a satellite link for distribution across the internet, as demonstrated in Figure 11. 


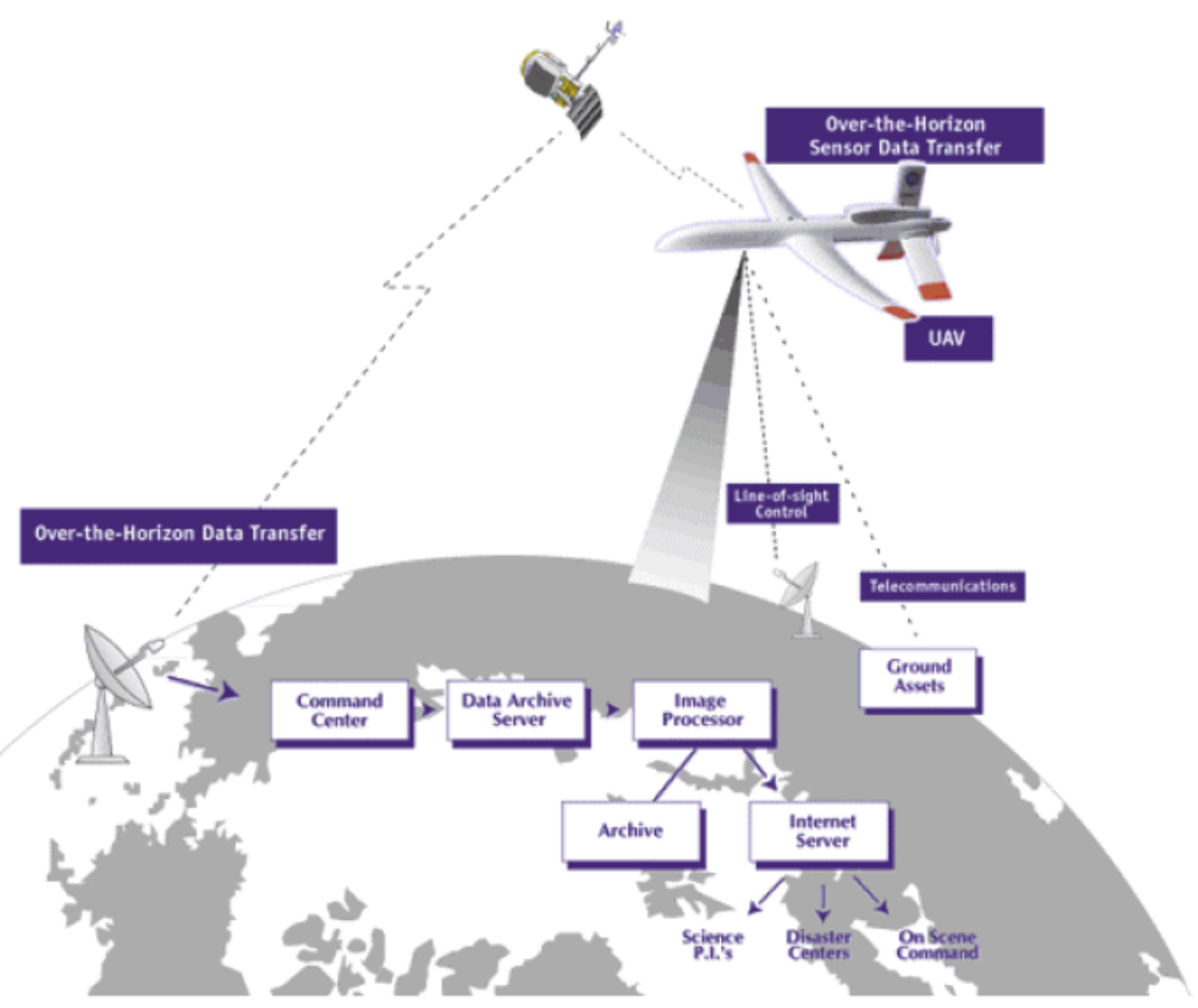

Figure 11: Example of HALE Fire Mission Data Distribution ${ }^{17}$

A Low Altitude Short Endurance (LASE) aircraft was also proposed for wildfire response efforts under the belief that a low altitude aircraft, LASE UAV, could be used to provide on-scene situational awareness to the firefighters on the ground. The aircraft would then be deployed locally and launched toward a specified wildfire to follow the perimeter. As part of this effort, the LASE vehicle would monitor fuel status and be capable of returning home through a computed optimal solution. This would be treated as a separate mission; however, the LASE aircraft could be tasked using the HALE generated information. ${ }^{19}$ 


\subsubsection{Problem Definition}

Starting with the initial concept of operations defined as part of the NASA and USFS partnership, examinations set forth below seek to refine the HALE and LASE wildfire detection and response architecture from the original "straw man" layout, which includes identifying the driving requirements for the wildfire missions and refining the operational architecture to meet these USFS needs. The core issues of communications, sensors, and necessary algorithms are then addressed for both the HALE and LASE aircraft. Next, the algorithms are prototyped in simulation to demonstrate feasibility of deploying this system in the field. Future work is assessed with each simulation to determine the next steps for providing for a proof of concept in a non-linear and realistic environment. 


\section{WILDFIRE MISSION DESCRIPTION}

One of the most resourceful applications for UAV introduction into the civil sector is disaster detection and response. Along with several countries including Japan ${ }^{1}$, Canada $^{21}$, and Australia ${ }^{22}$, the United States has also adapted these vehicles from their original military purpose of detection and tracking to their civil side equivalent. Due to their airborne capabilities, these vehicles operate in a wide range of missions, including hurricanes, floods, earthquakes, tsunamis, droughts, and wildfires. ${ }^{21}$ These missions combine the need for lengthy surveillance with rapid deployment once disaster strikes. UAVs meet basic standards with their "dull, dangerous, and dirty" philosophy while supplying the benefit of not risking a human life within the hostile environment itself.

Within civil sector applications, land management has especially noticed the promise of what UAVs can do for wildfire detection and response. ${ }^{21}$ Wildfires are a complex problem for the USFS thus leaving the department constantly seeking new means of combating these disasters. Many of the problems in fighting these blazes stem from the fact they are seasonal, but the location of where these massive fires occur remains unpredictable. Additionally, once wildfires occur, they are a volatile and changing environment. This makes effective ground surveillance difficult and periodic updates from available satellite systems of limited use. Locally controlled airborne sensors can provide constant updates while remaining at a safe distance from the situation. $^{21}$ 
Due to these capabilities, many American agencies are currently working to integrate UAVs into the USFS. This effort has included the participation of major aircraft manufacturers, research agencies, and academia for the purpose of addressing technical issues with UAVs in their current state. ${ }^{1}$ Though research is directed toward bringing the necessary technology online for UAV integration into wildfire response, it unfortunately means that little research has been done regarding how they will actually be deployed. In order to address this issue, sample deployment architecture is outlined to demonstrate how UAVs can be integrated into the existing USFS organization. This overall design is done with an emphasis on the actual daily detection, mitigation, and wildfire response. While this prototype architecture is outlined to meet the specific needs of the USFS, it should be noted that the design also serves as a template regarding how parent-child UAV architectures can be utilized to combine the strengths of any number of assets for a common goal.

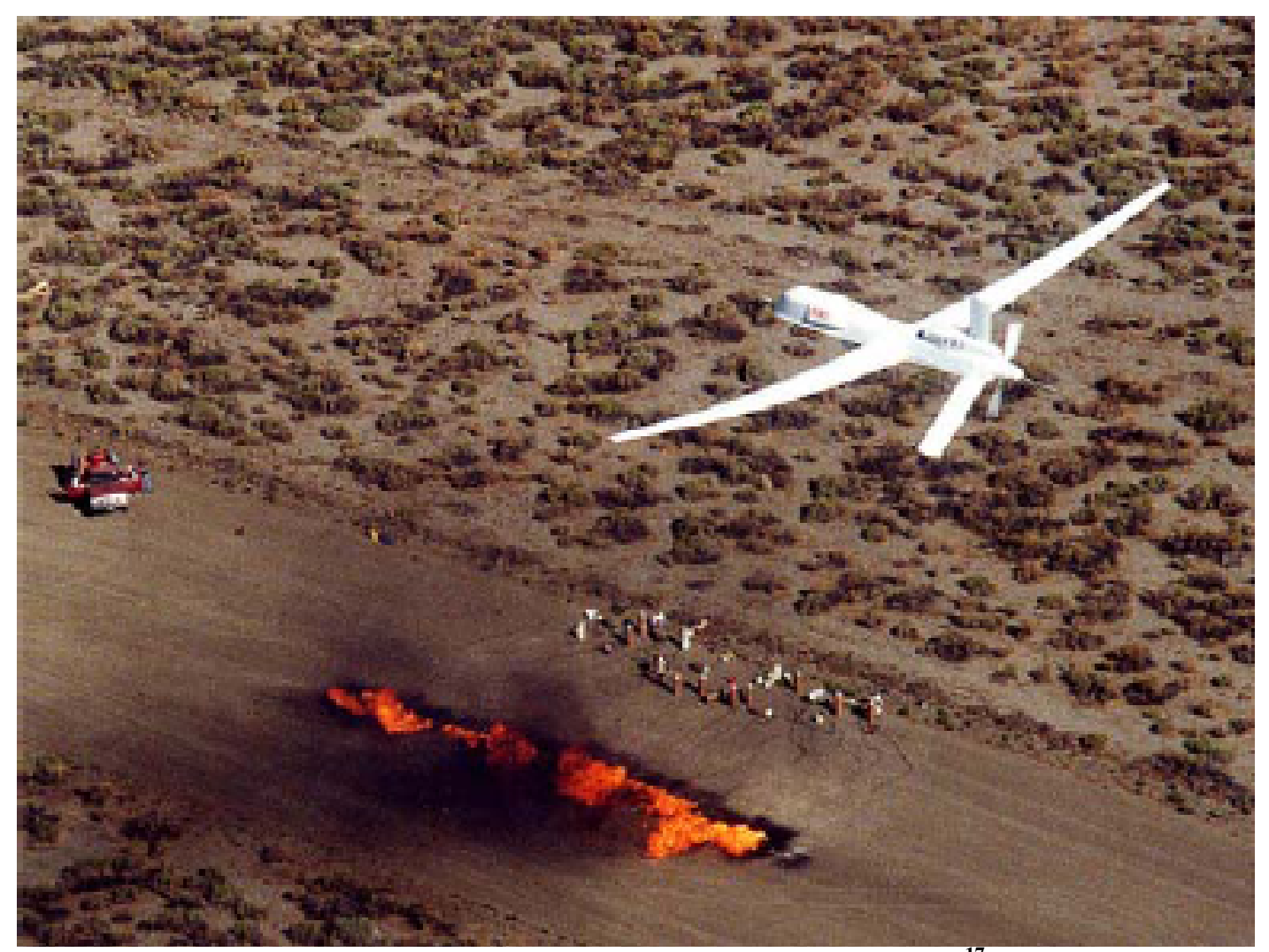

Figure 12: ALTUS II Flight in FiRE Demonstration ${ }^{17}$ 


\subsection{Mission Constraints}

The first step to designing any feasible UAV architecture is to identify the mission to be performed as well as operating constraints to be imposed. Many of these operating constraints are derived from the USFS ultimate goal of providing quick decision making to disaster managers during hazardous events and improving the incident team's support structure. From this overarching theme, several operating requirements can be derived in terms of data gathering, communications, supported region, and mission timeframe. The USFS has expressed needs for how each of these categories should be fulfilled. Additionally, there is the desire to incorporate research that has been completed, or is currently underway, with various research groups, agencies, and universities.

\subsubsection{Aircraft Communications}

Traditionally the USFS has utilized line-of-sight (LOS) communications for their wildfire operations. While this has proven reasonable in the past, as remote sensing capabilities are added to the catalogue, the need for greater information distribution is presented. An example of this includes relaying information from the scene of the wildfire to the National Interagency Fire Center in Boise, Idaho. ${ }^{23}$ As a result, research is exploring the usage of over-the-horizon (OTH) communications via satellites or the internet. The ability to add and utilize these communication capabilities is a must for any future UAV implementation which becomes a problem when noting

that the information dispersal requirement needed to consider interoperability. ${ }^{18}$ Any form of information relay must come in a standardized format so data can be shared without proprietary protocols between the command stations, incident commands, and other resources tasked to fight 
the fire. A flexible communications standard is then implied which can be utilized on a variety of platforms for a wide range of end users.

The enhancement of the type and quantity of data sent through these communication links also poses as a strong need for the USFS. This includes transcending standard telemetry and moving to support voice relay, video, multiband imagery, and other sensor data in real-time. ${ }^{17}$ As a result of this design driver, any UAV architecture would need to support imagery, video, voice, and a data channel to provide a means of controlling the autonomous vehicles. In addition, the USFS has dictated that this information must be provided within a 15-minute window to minimize potential loss of resources, personnel, and property as specified by the USFS. ${ }^{18}$

Along with these design requirements, the looming factor of cost is always present for any architecture which is especially true with the need to transition towards OTH communications. Currently satellite bandwidth is sold in two forms: a yearly lease for a specified bandwidth amount or per bit transmitted over the communications satellite network. While a yearly lease is the typical means of ensuring cost-effectiveness when dealing with large amounts of data, the seasonal nature of wildfires means large amounts of money will be wasted as the connection sits idle. Additionally, the usage-based metric can accumulate cost quickly for large data sets and is inefficient if the line is heavily used. These two options must be taken into consideration if the architecture is to be implemented on the national level. 


\subsubsection{Civil Airspace Restrictions}

One of the most significant problems plaguing any operation in the United States is access to the NAS. Under strict regulation by the FAA, the agency demands that while flying, any remotely piloted or autonomous vehicle must have the same capabilities as any manned aircraft passing under air traffic control. This includes the ability to communicate with air traffic control, visually avoid close proximity aircraft, stay out of restricted airspace, and file flight plans for long duration flights. ${ }^{1}$ In each restriction, the setbacks for autonomous flight are apparent since all of these regulations are geared for manned aircraft operations. There is also very little flexibility in these regulations considering the amount of commercial traffic that operates above the United States as noted in Figure 13.

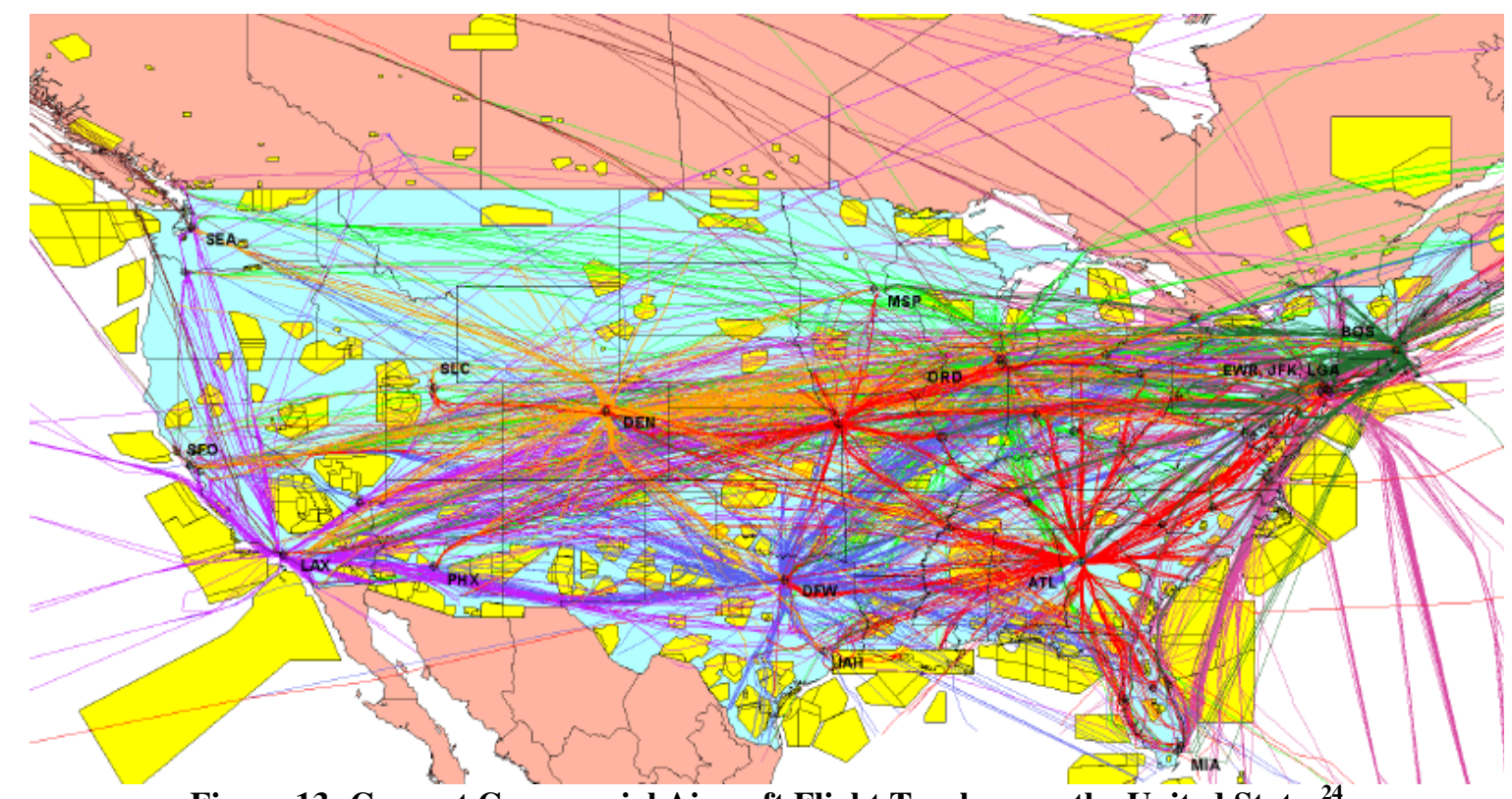

Figure 13: Current Commercial Aircraft Flight Tracks over the United States ${ }^{24}$

The consideration for how to operate within the NAS is important since any detection and response missions will need to fly over the United States. Due to wildfire missions predominantly occurring in the western United States, the USFS has expressed the desire not 
only to operate within the western United States, but to carry out prolonged missions within that entire coast as noted from Figure 14. Furthermore, the unpredictable nature of wildfires means a predetermined route is of limited use without the ability to modify plans and respond to new occurrences which leaves essentially three options when dealing with the NAS: fly within the NAS under the FAA regulations, fly above controlled airspace, fly below controlled airspace, or operate locally while the airspace is restricted for the wildfire.

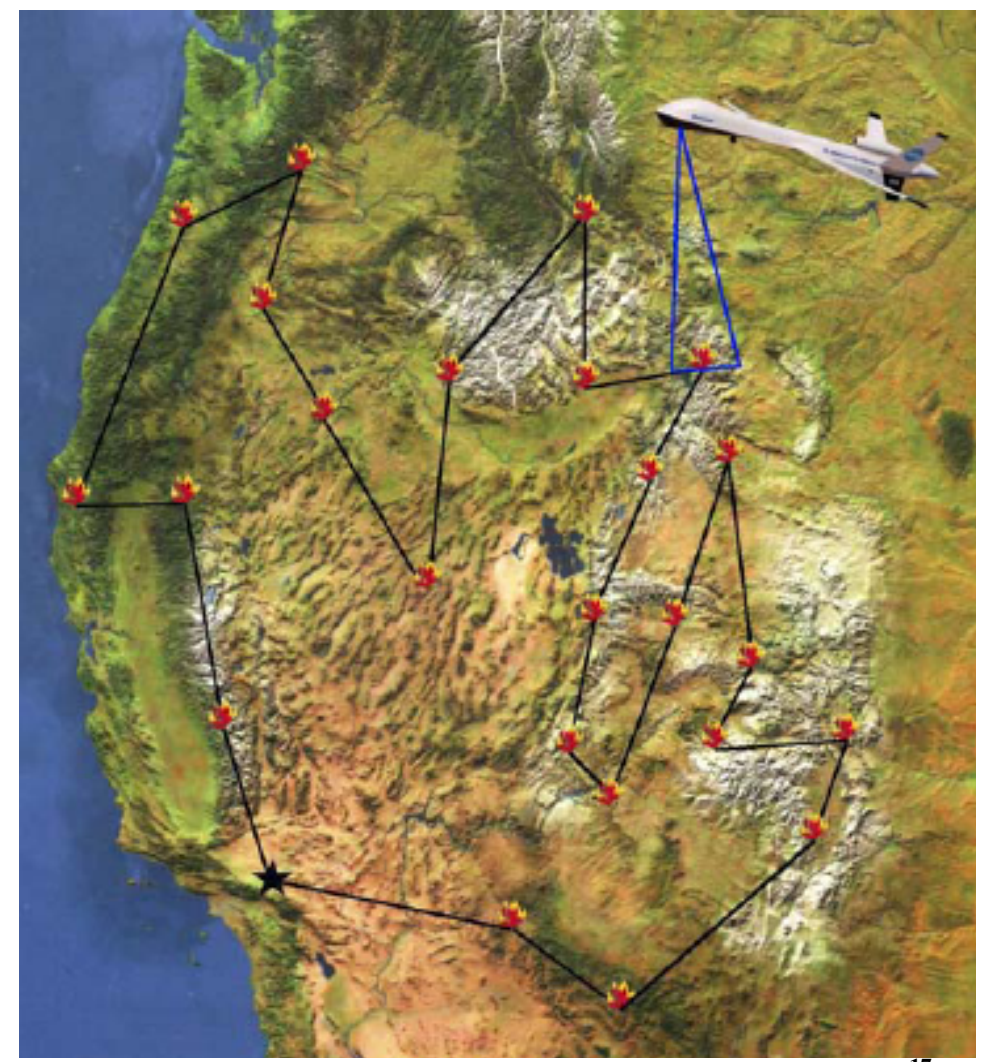

Figure 14: ALTAIR-Western States Fire Mission Outline ${ }^{17}$

Examining the four options above, attempting to gain continuous access to the NAS would require an array of sensors and a lengthy development timeline to develop the required see-andavoid technologies. Additionally, the file-and-fly methodology would create an inability to adapt the route if emergencies occur due to the FAA requiring strict adherence to the planned profile despite detected events. This would directly conflict with the desires of the USFS depicted in 
Figure 14 and push the timeline for practical implementation out by multiple years. However, the option to operate locally would also violate the USFS's wishes for wide area surveillance and support for long distance collaboration, as well as, not providing any benefit beyond what the USFS already has obtained with manned resources. Extremely high altitude assets that operate above all forms of restricted airspace can fulfill a majority of the mission objectives but are exceptionally costly with no local support unless a network of these UAVs are used. A combination of all these options may serve as the most likely means of meeting all mission objectives.

\subsubsection{Sensor Payloads}

Among the category of sensors, the USFS has done considerable research into what types of sensors have proven, and will consistently prove, useful in natural disaster detection and response. As the primary objective is to gather data, the USFS has requested that infra-red data be the baseline collected to determine a fire's state. Unfortunately, this information is of limited use for spread prevention since thermal imagery does not determine the state of the local region, vegetation, or the fire's movement. In order to obtain this data for natural disaster deterrence, the USFS has further requested that multi-spectral data be collected with the ability to add additional sensors as technology matures. ${ }^{18}$ A listing of sensor types and the capabilities they provide in aiding detection and response is provided in Table 1. These sensors must operate in the given environment chosen for them, which can range from a wildfire environment with poor visibility, extreme heat, and toxic atmosphere, to high altitude reconnaissance with severe cold, very little air, and atmospheric interference with measurements. 
Table 1: Sensor Types Available For Fire Detection and Response ${ }^{21}$

\begin{tabular}{|l|l|}
\hline \multicolumn{1}{|c|}{ Surveillance Sensor } & \multicolumn{1}{c|}{ Principal Functions } \\
\hline Real Aperture Radar (RAR) & All-weather surveillance \\
\hline Synthetic Aperture Radar (SAR) & High resolution imaging \\
\hline Moving Target Indicator (MTI) & Detection of moving targets \\
\hline Electro-Optical & High resolution imaging \\
\hline Infra-Red & Thermal detection, Thermal imaging \\
\hline LIDAR & Targeting, Ranging \\
\hline Meteorological Sensors & Meteorology \\
\hline
\end{tabular}

While this data serves as remote sensing augmentation for the USFS, the data is of little or no use unless the collection location is identified, which requires all data collected to be georectified. ${ }^{23}$ The alignment of the image to the vehicle, and to a GPS coordinate, will allow the data to be of use to both the aircraft itself and the firefighters on the ground fighting the blaze. This dictates the need for inertial measurements and embedded GPS which allows for ground imaging data collected by the aircraft payload to be overlaid on any map.

Payloads are the main driving force behind aircraft selection which is the first consideration of sensor selection. Typically, UAVs are designed for a particular mission. However, the choice of an aircraft that is best able to meet the payload and performance requirements among existing platforms is the most feasible and low-cost option. As can be seen in Figure 15 below, the total payload weight cannot exceed 1,200 pounds; otherwise it will exceed the carrying capacity of any listed UAV. ${ }^{25}$ Based on the NASA developed Airborne Infrared Disaster Assessment System (AIRDAS), the sensor and mount weight total to 270 pounds. ${ }^{26}$ Using this value as an absolute minimum payload weight, the gross weight of the aircraft required to carry this payload is estimated at 1,578 pounds based on the empirical relationship established below in Figure 15. This eliminates the possibility of dropping in-situ sensors or smaller aircraft to collect higher 
resolution data since the estimated aircraft weight to carry this as a payload is 9,222 pounds and well beyond the capabilities of any reasonable platform. Additionally, the established weight and size requirement will ultimately limit the number and type of sensors the aircraft is capable of carrying.

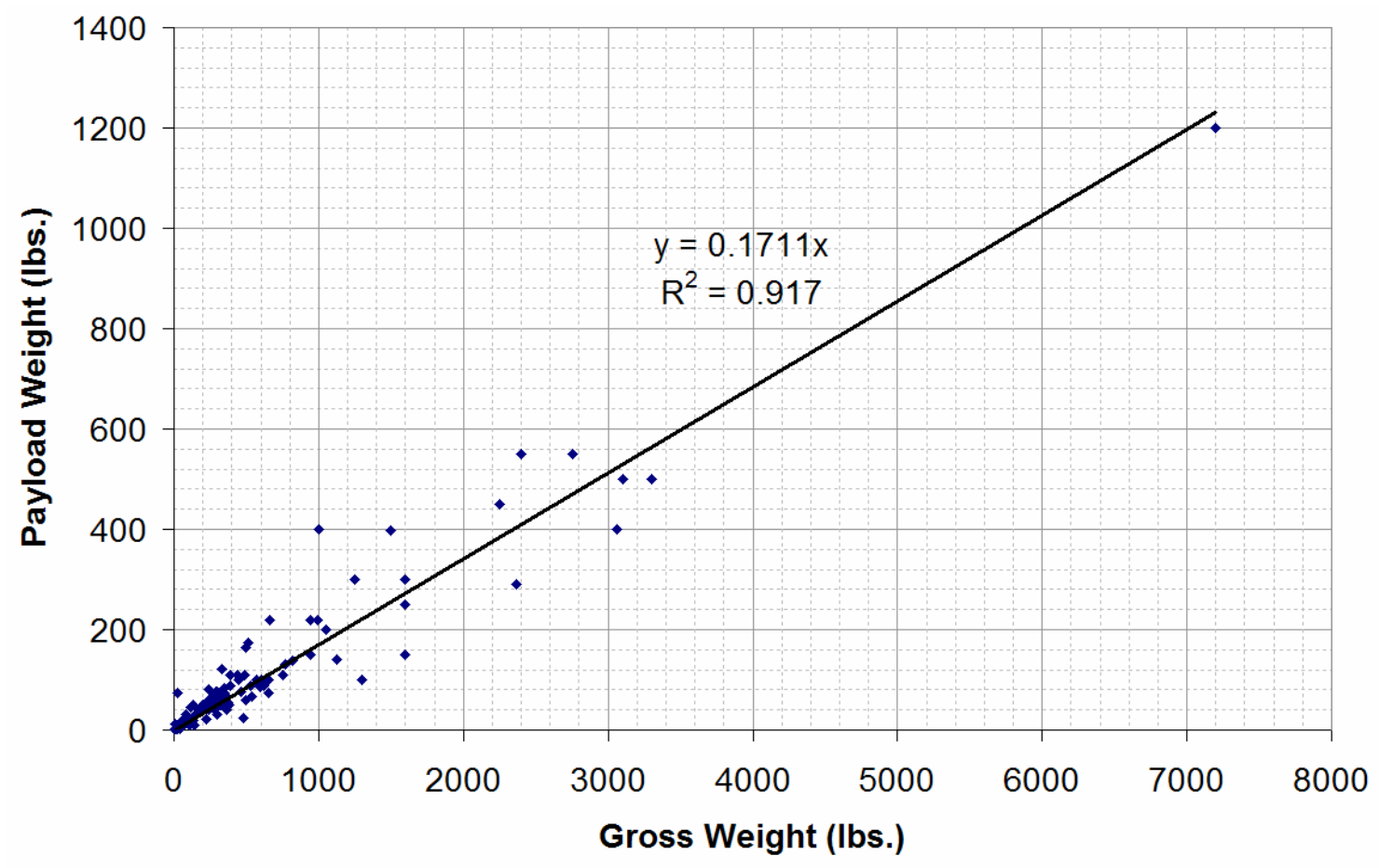

Figure 15: UAV Payload versus Gross Weight Trend from UAV Data ${ }^{25}$

The next consideration for sensor selection becomes a tradeoff between the number of sensors, the aircraft range, and the frequency of data collection which becomes especially true when considering the mission scope and data communication requirements for carrying out the prescribed wildfire mission. In this instance, the necessity to patrol the entire western United States implies that a long endurance aircraft be utilized with OTH communications, requiring long range and high resolution imaging capabilities. ${ }^{23}$ However, this directly conflicts with the secondary objective of the mission that dictates the vehicle must maintain high frequency updates of the situation once a fire has been determined. Within this particular application, a 
variety of payload requirements that is paramount to gaining access to the NAS, such as radar and $\mathrm{OTH}$, become cost inefficient for a localized resource. Attempting to design for both missions with expectations of using multiple aircraft with duplicate sensor capabilities will not only increase the number of sensors, the payload weight, and the vehicle range, but ultimately the vehicle weight and cost. Using the practice of specializing to missions, the missions can be split into local and long range reconnaissance missions with aircraft designed specifically for each.

\subsection{Mission Concept of Operations}

Military development of UAVs has traditionally been tactical in nature, as UAVs are required to adapt to rapidly changing scenarios. Conversely, civil sector and research UAVs have typically leaned toward background observance and the examination of trends over long periods of time. The proposed research advocates blending these two concepts, one High Altitude Long Endurance (HALE) and the other a Low Altitude Short Endurance (LASE), under a common parent/child architecture, which would combine high resolution, in-situ sensing capabilities of a local resource with the substantial communication, payload, and regional awareness advantages provided by a HALE aircraft. Essentially, these two aircraft variations would act as one and not only exchange data, but actively react to each other's needs in order to optimize mission completion and information collection time.

The scenario begins with the USFS selecting locations of reported wildfire outbreaks throughout the western United States from a display similar to the one shown in Figure 16. The user would outline any number of polygons symbolizing areas requiring observation with the final 
information then relayed to a HALE UAV ready for lengthy deployment. Once received from the USFS command station, the HALE utilizes its onboard adaptive planning engine to create a selfoptimized route through each specified search location. Next, the generated plan is relayed back to the command station for final confirmation. After confirmation to proceed is given by the station, the HALE ascends to an altitude of 100,000 feet and patrols the route to which it has been assigned.

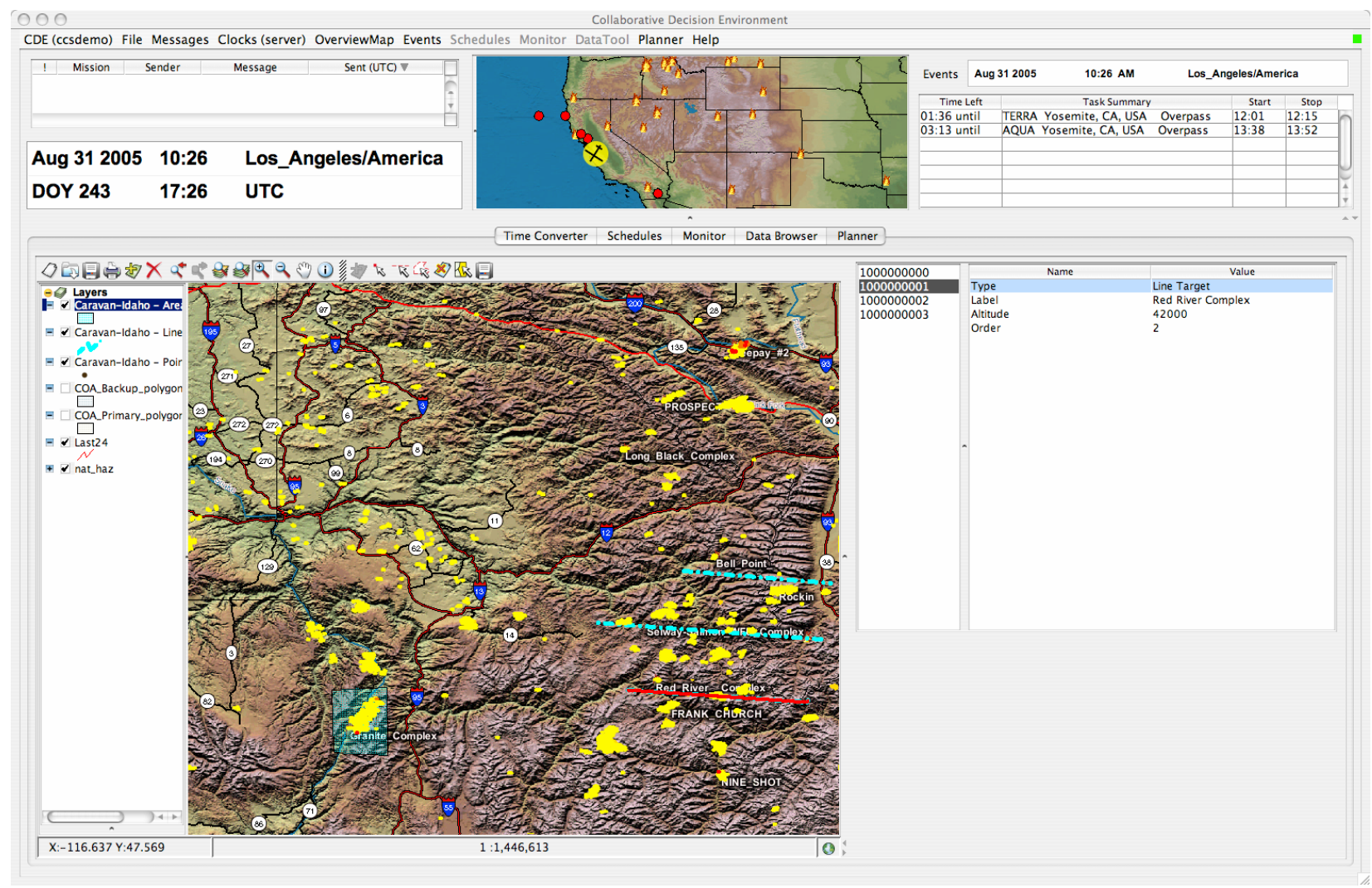

Figure 16: Collaborative Decision Environment Displaying Live Wildfire Data ${ }^{20}$

Using its onboard multi-spectral cameras, the UAV will arrive at each target and map the userspecified region. While processing the sensor data, the UAV will also examine the incoming data streams for a spike in the infra-red (IR) band that is characteristic of high temperatures. If this wildfire signature is detected, the HALE will contact all stations responsible for the region and query what resources are available to examine the phenomena. Each local ground station will 
then respond to the HALE by identifying its LASE class resource, the capabilities of the vehicle, and its current status.

The HALE UAV, making use of the onboard planner and the self-acquired knowledge of the situation, will proceed to allocate the resources to quickly and efficiently examine each instance. Incorporating factors such as no-fly zones, terrain elevation, identified target locations, and LASE vehicle limitations (e.g. cruise speed, cruise ceiling, and range), the HALE will generate an optimized plan for each LASE resource capable of handling the situation. The HALE will then transmit this plan to each LASE aircraft and responsible ground station for final approval. After the operator is satisfied with the plan, making any required modification, the LASE is dispatched to verify or map the instance in high resolution using its onboard sensor package. The final path can then be repeated as necessary to monitor the progress of the detected wildfires thus providing rapid information updates to firefighters deployed on the ground. The flow of information for the architecture is depicted in Figure 17. 


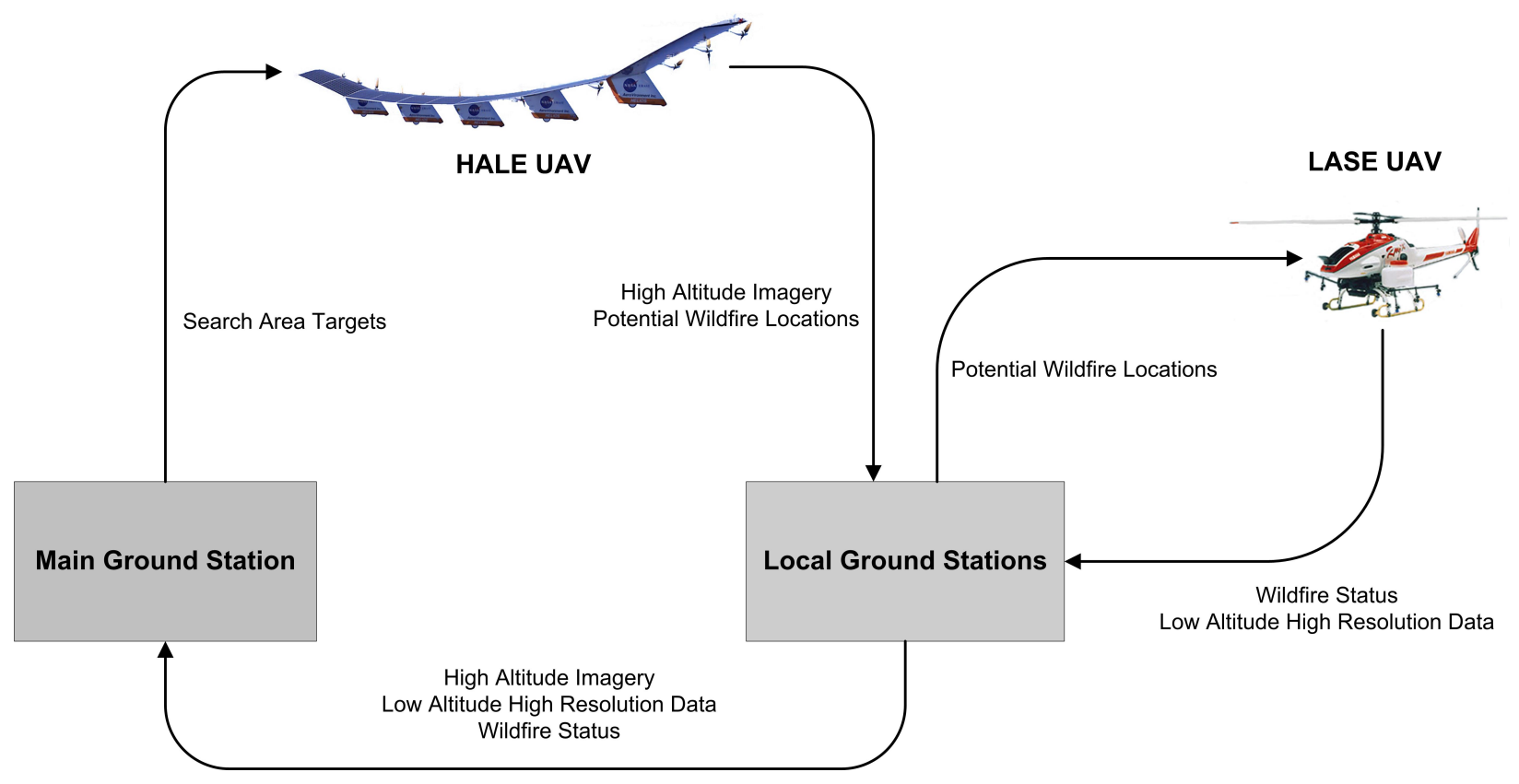

Figure 17: Proposed HALE and LASE UAV Wildfire Architecture

The search region overview and close proximity data collected from both the LASE and HALE aircraft is collected at the local ground station. An internet connection is then utilized at the local station to close the loop on the information gathered from the UAVs and relayed to the USFS central command. This situational data can then be further processed and distributed to other agencies for planning and resource allocation decision making over the long-term fight against the fire. This provides an extremely cost effective solution compared to transmitting the data back through satellite which is both limited in bandwidth and expensive.

\subsubsection{HALE UAV Aircraft}

The HALE-class UAV's main function throughout the mission is to act as an information gathering point and mobile base station for operations. Opposed to a master command center concept requiring a ground station, this practice ensures that all data products acquired are delivered directly to those responsible for that region which alleviates the minor latency and 
bandwidth limitations associated with satellite communications by distributing large data products over close proximity and reliable communication networks. In addition, the HALE actively participates in the mission whereas in most other operations it would merely perform reconnaissance work. This is an evolutionary next step for HALE vehicles since at any moment it retains the largest portion of situational awareness. The onboard planning capability coupled with this wealth of information makes it a natural candidate to plan the reactionary phase of the mission. The HALE alone would require sophisticated autonomy, high resolution sensors, and long-endurance aspects in the wildfire missions which eliminate the need to require the same level of financial investment in the LASE vehicles. Effectively, a scenario is created where the LASE platforms can differ greatly, be relatively inexpensive, and perform this task in addition to being tasked for other fire fighting needs.

Since HALE class UAVs of this caliber are still in the developmental phase, much of the performance and design of these vehicles is still heavily conceptual. As a result, a tentative set of capabilities including a month-long deployment at a 100,000 foot cruising altitude HALE is proposed. ${ }^{23}$ These numbers are an extrapolation based on solar-powered, high-altitude, and longendurance UAVs being developed by many countries as self-sustaining low-earth orbit (LEO) satellite replacements (i.e. pseudolites). While these numbers are unrealistic for present systems, the architecture is resilient enough to be implemented immediately by supplementing HALE endurance for quantity.

The necessity for this type of aircraft is evident considering the issue with UAVs accessing the NAS. As a result of FAA restrictions and the aircraft's inability to communicate with air traffic 
control (ATC), aircraft that must avoid commercial traffic may do so by simply ascending above it, which is performed by spiraling up to the final cruise altitude until sparsely used Class E airspace is reached, and leaving the vehicle free to fly to its predetermined location. Relaying on the vehicle's ability to access higher altitudes plays a dual role by benefiting data collection and allowing the aircraft to operate within normally restricted regions. Additionally, by limiting the takeoff and landing locations to restricted military airspace, the path these aircraft take can bypass hazardous oncoming aircraft since their presence is accounted for and air traffic is rerouted accordingly. An example how UAVs avoid commercial airspace is demonstrated in Figure 18.

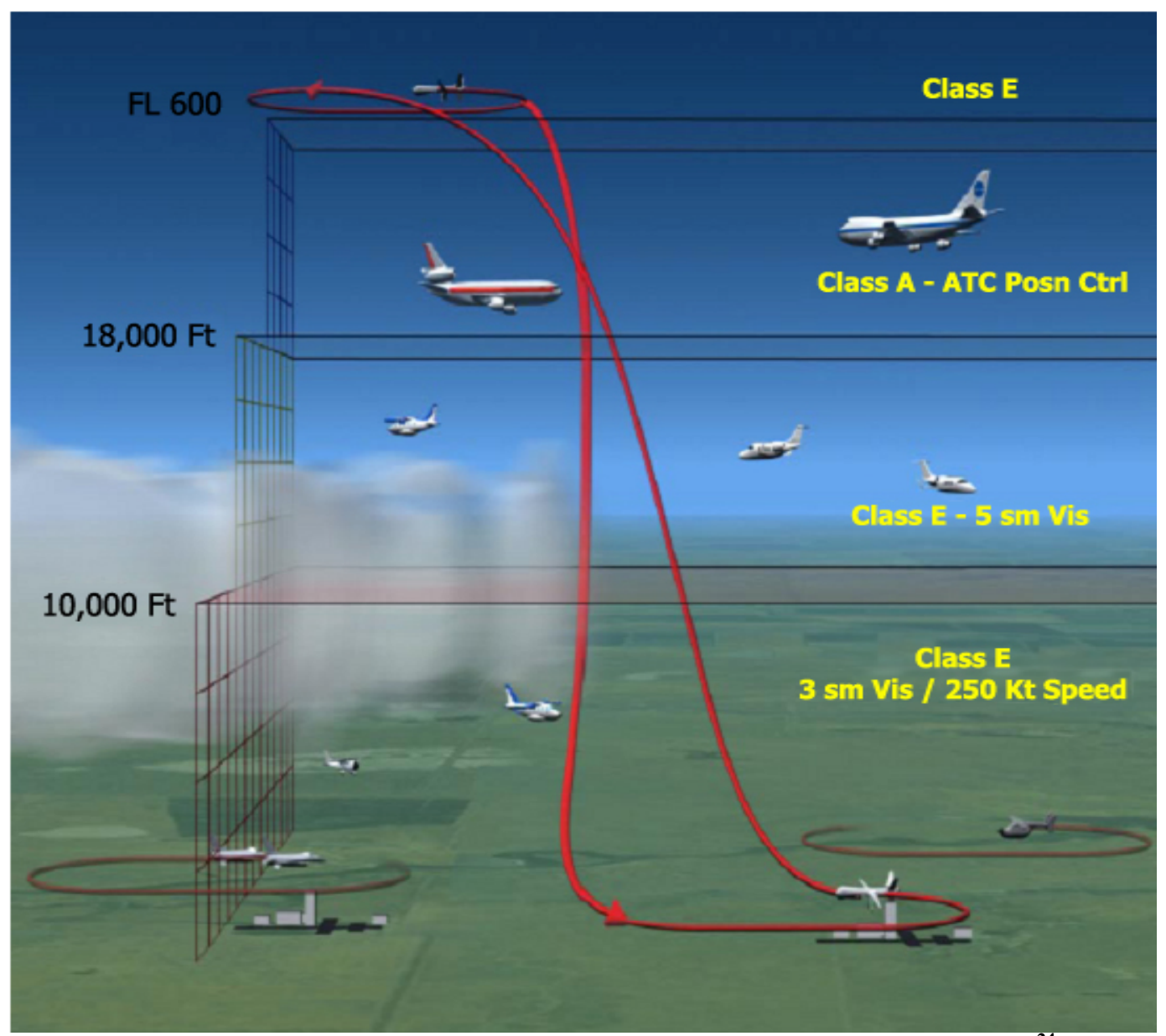

Figure 18: Common High Altitude UAV Ascension Path to Cruise Altitude ${ }^{24}$ 
As the HALE UAV has a unique high-altitude vantage point, the opportunity for a wide array of onboard sensors is provided. Potential combinations include LiDAR, Multi-Spectral Imagers, Hyper-spectral Imagers, and Synthetic Aperture Radar (SAR) since they are proven technology for the harsh environments at altitudes in excess of 50,000 feet. For this particular wildfire mission, the infrared band proves useful as it allows thermal image mapping. ${ }^{21}$ As derived from the USFS requirements however, detection must be combined with additional imaging bands to provide a prevention capability. Multi-spectral or hyper-spectral imaging sensors serve the ideal solution for this mission, such as the example shown in Figure 19.

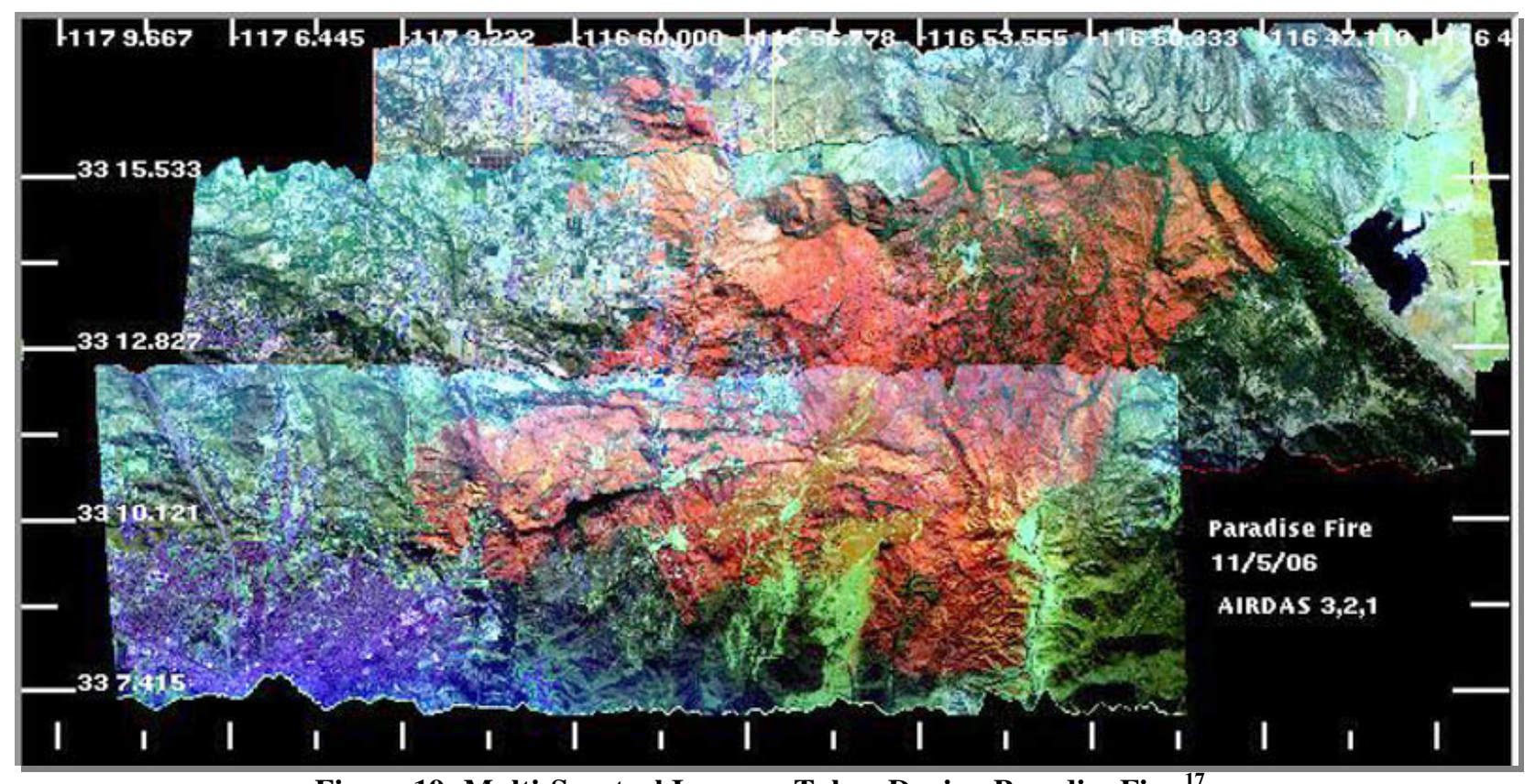

Figure 19: Multi-Spectral Imagery Taken During Paradise Fire ${ }^{17}$

Unfortunately, attempting to integrate this sensor into the vehicle airframe would require a new airframe designed around the capabilities, which subsequently delays the implementation of this architecture and dedicates the aircraft entirely to the mission at an exorbitant and unnecessary cost. By integrating this functionality in a payload pod attached to a hard point on the UAV wing the above discrepancy can be eliminated which would allow any aircraft large enough to support external pods to contain the system and create a self-sustaining system that can be linked into 
any HALE UAV. Along with the sensor, the processing capabilities will need to be provided to allow the pod to acquire the image, geo-correct it via the onboard EGIs, and determine if fire locations are present.

While development of sophisticated planning environments are already underway, the incorporation of vehicle dynamics and performance constraints for the LASE tasking will be a central theme. Due to the difficulty in adaptively planning and tasking arbitrary LASE vehicles. Various algorithms will be first investigated for the HALE UAV to better ascertain their viability at solving the Traveling Salesman Problem (TSP), locating the most efficient way of visiting all desired locations and then returning to the origin. Using this data, a comparative analysis of various approaches will be performed that accounts for computational time, overall score, and possible comparison to human results. Once a relevant algorithm has been confirmed, a baseline planning agent will then be implemented as the environment for testing the impact of non-linear aircraft and environmental behavior on route optimization, which will include modeling aspects such as turn radius, spiral descent requirements, fire danger, and wind effects in the adaptive planner. Once HALE algorithms are developed and handled sufficiently, the LASE tasking algorithms will be considered to ensure no-fly zones, vehicle limitations, topography, and air traffic are then considered. After this is completed, an attempt at syncing the plans for the two aircraft will be examined to account for communication radii, information relaying, and plan updates.

The most critical and valuable asset provided by a HALE aircraft of this form is the capability of acting as a communications relay, thus replacing satellites. However, this has not been fully 
researched for application by a HALE aircraft and therefore multiple aspects of this topic must be examined to ensure the HALE does not become a single point of failure. A study centered on link type, signal strength, and frequency band would be necessary to evaluate whether or not a HALE could not only collect the data, but consistently relay data using its natural OTH capabilities. Also, along with the HALE's link to the ground station, factors influencing the LASE link quality and type will prove equally important. Both will be studied within to the scope of the concept of operations to ensure successful and reliable mission completion.

\subsubsection{LASE UAV Aircraft}

While the HALE serves the primary decision of making roles within the parent-child framework, this does not diminish the importance of the LASE aircraft in the mission's completion. A parent-child architecture has a unique; while the parent HALE vehicle is rigorously defined by its payload, communication, and planning needs, it frees the LASE to be more dynamic and flexible. By placing the burden of planning and maintaining consistent communications on the parent, the child LASE UAV is capable of being any level of autonomy, cost, capability, and platform type. The LASE, taking advantage of this freedom, can be free to carry out other assigned tasks once the HALE's tasking is complete, which allows easy incorporation into any existing firefighting unit using UAVs. The singular investment of a low level fixed wing or rotorcraft can be reused or reequipped for other purposes yet still fall under this framework. The local USFS station is also made aware of region wide situations through the HALE without having to blindly send out the LASE to locate the fire instances itself which minimizes cost dramatically and makes owning a LASE a feasible option instead of customizing the platform to hold expensive payload and tasking it for dangerous missions. 
Considering the LASE UAV can serve any purpose so long as it is capable of performing actual fire identification, the payload becomes a question of finding the most useful sensor package. This can range from anything from visible camera and optical IR cameras to gas chromatographs and laser range finders. While the actual payload capacity will be smaller, the flexibility of changing payloads to perform various other missions is granted. This means once an investment to obtain a UAV is made, the vehicle is not locked into the singular role of reconnaissance as the HALE. The LASE UAV is assumed to have a forward-looking IR camera with a wide field of view as a minimum for sensing capabilities. As a baseline for the wildfire mission, enough processing capability is required for low level autonomous flight including waypoint navigation by means of a simple autopilot. The onboard computer will send signals to the autopilot based on information from a GPS (Global Positioning System) and strap down IMU (Inertial Measurement Unit).

While the HALE aircraft will be responsible for the path planning and route optimization processes, the LASE aircraft will also still require basic algorithmic development to ensure a certain degree of autonomy. As HALE have more information at their disposal, their role in the mission contains more of an optimization element while the LASE vehicles, which have typically worked in more of an information vacuum, are required to be more reactive in nature. Though the parent-child architecture seeks to bridge this knowledge rift by installing the LASE with low altitude maneuverability of a light aircraft and global knowledge of a high altitude resource, the LASE's operations demand a certain level of reactive response capability which 
translates into mandatory inclusions of algorithms, such as terrain following, obstacle avoidance, and waypoint following for mission accomplishment.

For this particular wildfire response mission, a simple algorithm must be developed to provide frequent updates on the condition of the fire, which is accomplished by creating an algorithm that allows smaller LASE aircraft to fly to a designated GPS coordinate to determine if a fire exists at the location. If a fire does exist, the flight control system must engage a simple mode to track the fire perimeter conditions. After tracking the entire length of the fire's edge, the aircraft returns to the local ground station, or point of deployment, to provide high-quality image data and compare the surveyed perimeter against the last known fire condition which allows for high-frequency updates and provides an invaluable and versatile asset for the firefighters on the ground. An additional facet for LASE UAV deployment is the realization that short endurance aircraft can enter a situation that overextends its means of returning home. Since the fire perimeter is undetermined at the time it is being tracked, an algorithm is necessary to allow the aircraft to return in the quickest and safest means possible when required. This algorithm is outlined later but works on the basic principle of using previously searched points to determine the quickest return route along the perimeter.

The communication aspect of the mission for the LASE will differ drastically from that of the HALE's role. Instead of a basis on maintaining the physical link, the LASE must aim for meeting the protocol requirements to ensure it can be tasked which this primarily supports the flexibility of the architecture. As a result, standardization of the message structure and ease of implementation becomes a heavy concern. In order to address these topics, a comprehensive look 
at middleware messaging schemes is proposed to see if standardization can be achieved through third-party software. After a brief description of the type of links encountered, a proposed distributive messaging framework and explanation of inner workings will be prepared to determine what the most likely implementation would be and how it would affect the communications. Emphasis on a commercial off-the-shelf (COTS) middleware product would also aid cost reduction and a comparison between this alternative and typical communication models would be warranted to see if any additional benefits are gained by the middleware approach as opposed to traditional communication methods.

\subsubsection{Ground Station}

As can be inferred from the concept of operations, both the local and command ground stations play passive roles in the entire series of operations. This is primarily due to the readiness level of current technology. As previously stated, the relaying of constant information is a great strain on any aircraft communication system, and sending the final product to the command center does not necessarily deliver the payload to the consumer directly. In this mission however, the HALE takes care of the tedious task of constantly scanning for fire signatures. The dull aspect of reviewing huge volumes of information results in an especially error-prone process with human operators. As the sensors become more capable, a greater burden will be placed on analyzing data quickly and dispatching it to the proper locations. The parent-child tasking ideology alleviates this problem using humans as an authorization point for the UAV-determined plans while automating the monotonous work of analyzing data and plotting courses. This allows the USFS to concentrate on wildfire prevention and response while leaving the task of detection and perpetual vigilance to UAVs. 
An interesting concept is proposed in this architecture, the ability to send data either over-thehorizon using the HALE platform or via the internet from the LASE base station. This allows for a definite advantage over standard single UAV deployments. Not only can the HALE double as a communications platform using its unique high altitude capability, but if extremely long range operations are required, the HALE can utilize the existing internet infrastructure during communications with the LASE stations which creates multiple data paths viable for the shipment of the final data product and does so while avoiding costly satellite networks and expensive additions to LASE vehicle capabilities. Critical to this architecture however, is the necessity of standardizing the communications syntax between these stations and the vehicles. By defining this method in a succinct way, a uniform means of tasking the UAVs is integrated into the vehicle and provided across all resources, without overloading the communication links.

Though the inner workings of the simulation planning engine and communications model are critical to the project's success, the interface construction for typical USFS usage cannot be overlooked. A look at how existing methods display the variety of data compiled by remote sensing vehicles may be required to ensure the technology can be utilized and maintained efficiently. If the interface to either the HALE or the LASE fails to captures the rationale for the reason a particular path was chosen, the ability for operators to alter or iterate the plans with the UAVs may prove to be the weak point in the overall system layout. Proper decisions cannot be made without the required information, and for that information to be used in the decision making process, it must be understood. Giving human operators the ability to modify target listings or paths is necessary to avoid duplication of efforts or dangerous scenarios. Conversely, 
if the information is not displayed properly and a misconception occurs, the LASE or HALE UAVs may be accidentally forced to remove actual fires or entire detection regions from their routines, defeating the point of the system. A graphical user interface (GUI) would need to be constructed to not only capture the decision-making cycle for the HALE's detection path, but also for the LASE's tasked path to ensure all operations and mission fulfillment are completed successfully. 


\section{WILDFIRE MISSION ARCHITECTURE}

In an effort to demonstrate these abilities, the aircraft algorithms will be prototyped along with a baseline communication method which will illustrate the flow of information through the parentchild architecture from wildfire detection to vehicle response. This example will explain vehicle autonomy by showing the HALE UAV iterating its route based on an initial series of reported fire locations selected by the base station operation. The HALE UAV will then search each target area utilizing its own situational awareness and information and relay those findings to smaller, more localized UAVs in the region. Next, a demonstration of basic communication with the ground station using COTS middleware is shown. Algorithms are presented for the lower daughter-ship UAVs that are capable of completing the lower-level reconnaissance and response tasks. When finally tied together, these should adequately prove that vehicle autonomy is capable of handling the detection and response mission with minimal human intervention. All the algorithms exist to perform this task, but parent-child architectures provide the ideal solution for communicating these goals down to the actual fire response team.

\subsection{HALE Path Construction Algorithms}

When considering the HALE vehicle's role in the architecture, the need to install decision making ability within the aircraft is imperative and becomes a requirement due to the duration of the reconnaissance mission, the need for route re-planning, and the avoidance of restricted airspace. Ultimately, the problem of searching for wildfires comes down to the optimization of a route through a series of search locations. Referred to as the "traveling salesman problem (TSP)," this classic problem is explained through the following example. A salesman must visit 
a collection of cities, but can only visit each city once. Also, the salesman must find the shortest route through all the cities and still return home at the end of the tour. While this challenge has been solved numerous times with a multitude of algorithms, the tackling of this problem with an aircraft, limited resources, and in real-time a adds new dimension to the predicament.

For the HALE aircraft, the TSP needs to be solved quickly and with minimal computing power eliminating many of the more elaborate solution methods, like simulated annealing or genetic algorithms and leaving a subset of solution methods that provide an initial tour, then optimizing the initial route until a suitable result is formed. Algorithms in this category fall under the greedy, insert furthest, and insert cheapest methods for the initial tour and two-opt and three-opt for the optimization routines. Each of these methods was compared and contrasted for its result and time required obtaining that solution to the TSP.

\subsubsection{Route Computation}

In order to compute TSPs, the determination of cost is critical. Typically, in two-dimensional problems, the cost is dependent on the distance required to travel from one city, or node, to the next. To allow the algorithm to perform its task, the distance from one node to all other nodes is calculated and stored in matrix form. A sample problem is provided in Figure 20 below with a set of 15 randomly generated nodes with the first used as an origin. This test case is randomly generated using a normal distribution to create a number between 0 and 100 . 


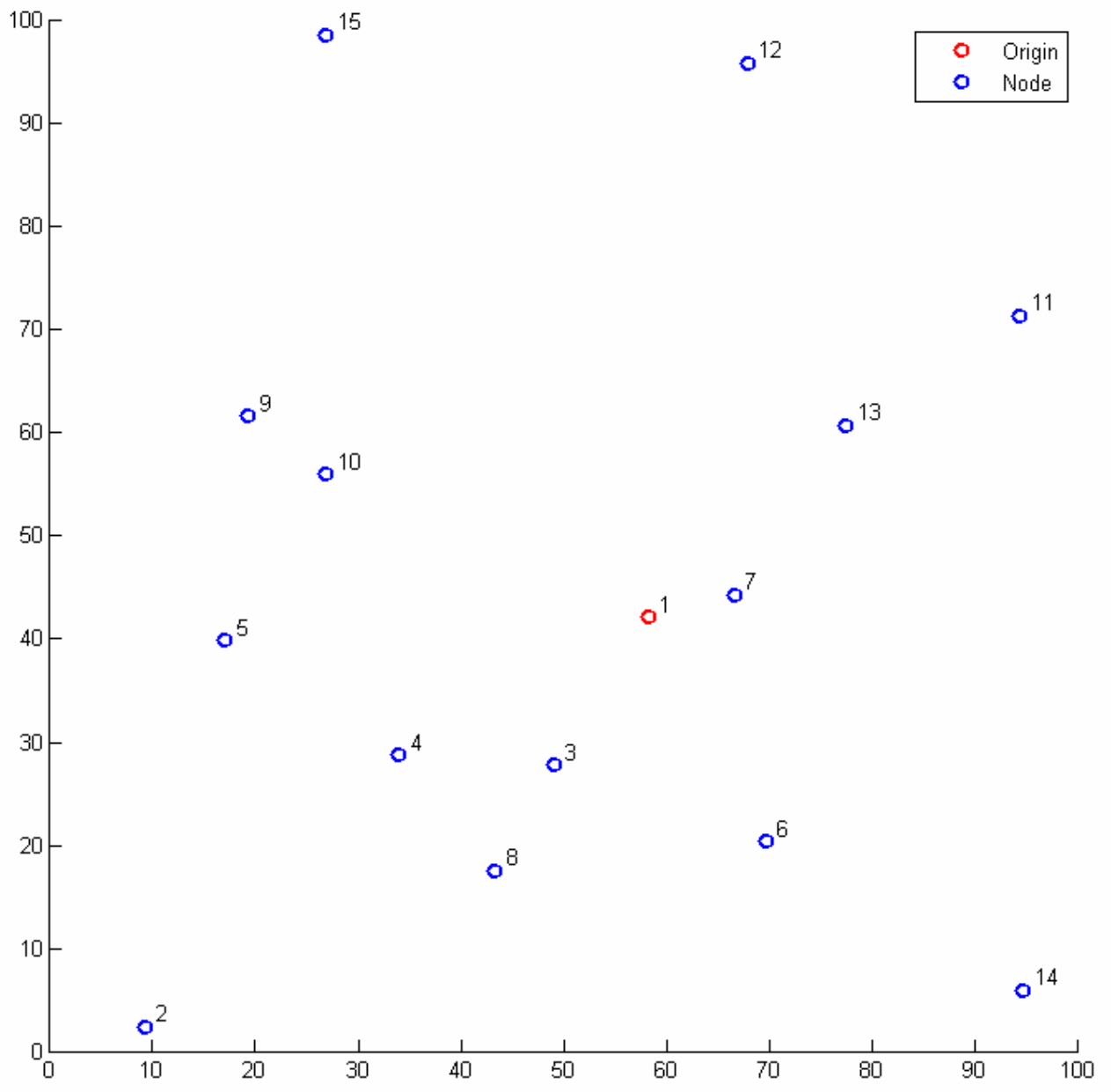

Figure 20: Sample Traveling Salesman Problem with 15 Nodes

Once the nodes have been created, the two-dimensional distance from each location to the other is computed for all combinations. Data is then stored in matrix form with the starting node as the row and the destination node as the column. For instances where the destination is the same as the origin, a zero is placed to show there is no travel cost associated with that combination. Also, since this is a two-dimensional problem with no added non-linearity, the cost associated with traveling to a node is identical to traveling back which results in a symmetrical matrix and can be treated as upper-triangular under these conditions. From this point, the TSP is solved by creating an, optimal or near optimal solution, using an initial route generating algorithm and is then 
subjected to improvement. For the 15 node problem cited above, the cost data is demonstrated in Table 2.

Table 2: Cost Matrix for Traveling Salesman Problem

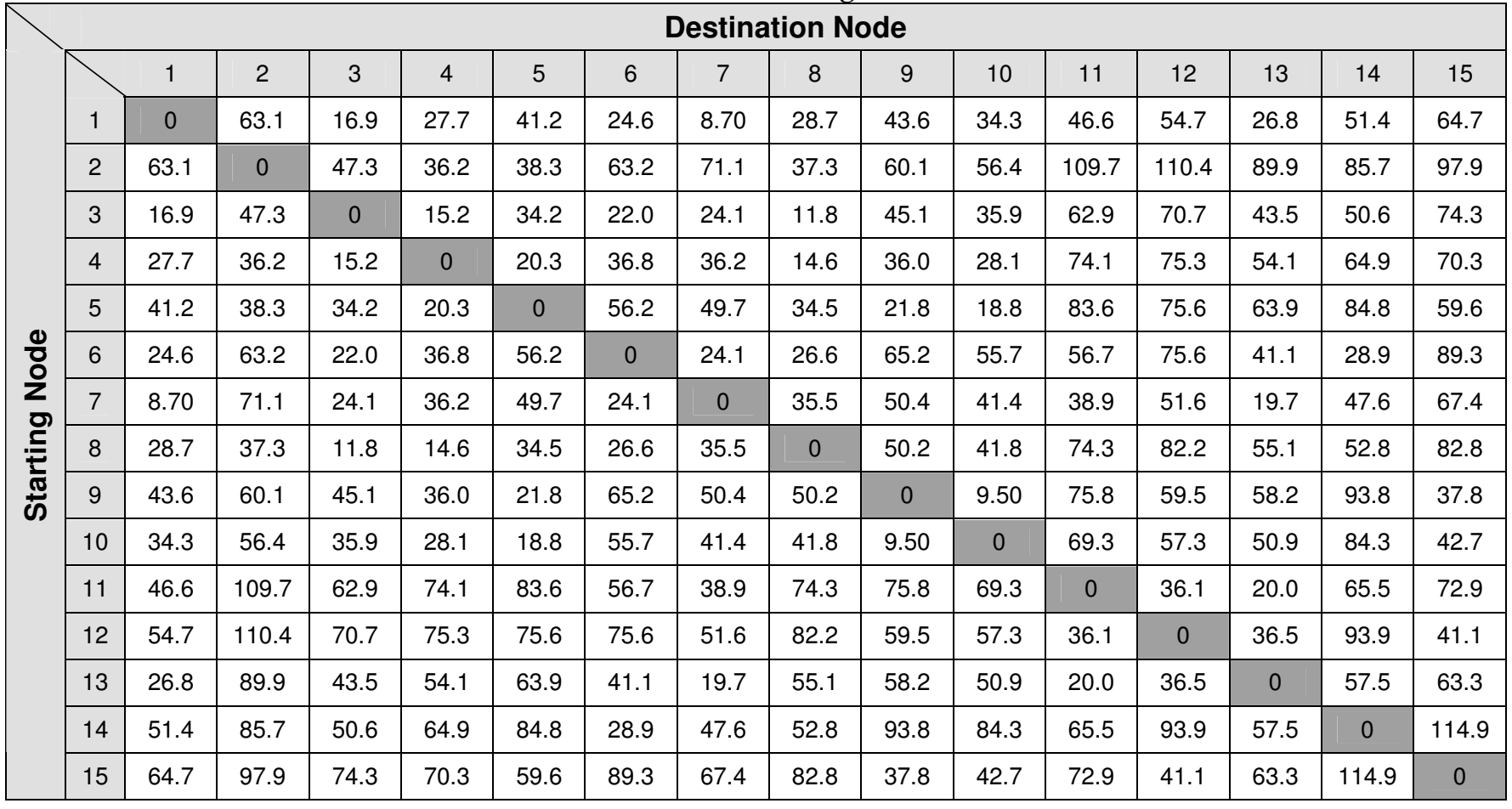

Each algorithm searches this matrix in a different means in order to locate the minimal cost for traversing a route. However, this cost matrix is not limited to distance to and from nodes and can be manipulated to reflect the non-linearity of solving route problems under realistic conditions. Along with a true distance to a target that incorporates the vehicle's flight dynamics, additional factors such as environmental conditions, proximity to nearby structures, no-fly zones, target urgency, and weather conditions can be incorporated. By factoring this information into the costing matrix, the information can be condensed in a relatively simple fashion allowing the UAV to make an educated decision based on current conditions. The next consideration is what the onboard aircraft planner will do with this information in determining its route. As a result, the task becomes a down selection of planning algorithms for the most likely candidate to provide optimal routing while accounting for vehicle limitations. 


\subsubsection{The Greedy Algorithm}

The greedy algorithm is a very simple algorithm in that it evaluates the cost of traveling to each city and chooses the lowest-cost move. This algorithm continues to choose the next lowest cost point until the only location remaining is the origin. Typically this algorithm initially provides adequate solutions. However, when the number of cities is large, poor results are obtained at the end when the greedy method is just adding the remaining nodes at increasing cost.

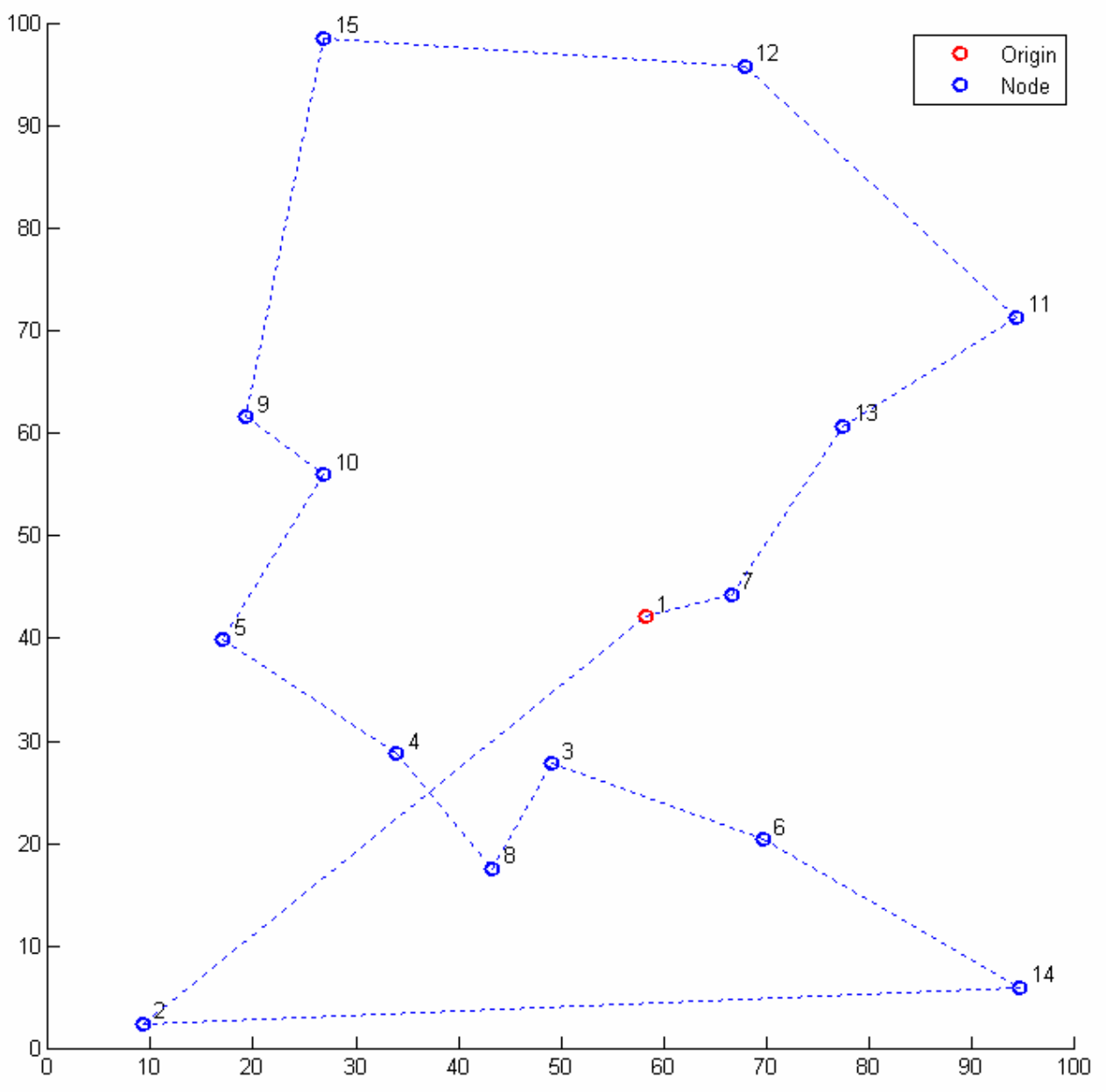

Figure 21: Greedy Algorithm with No Optimization

While the greedy algorithm is simple to implement, the procedure for computing the initial route potentially contains major drawbacks when working in a realistic environment. The first issue 
with this algorithm is that the algorithm is short-sighted and does not consider the selected node in context to the route or the remaining nodes. Essentially, a node is selected purely on a 'nextstep' cost basis. This algorithm has been proven to choose inefficient routes under certain conditions, such as clustering which can typically this solved with route optimization, but it proves successful when all nodes are selected and subjected to the optimization.

The second issue with the greedy algorithm stems from aircraft limitations due primarily to the greedy algorithm continuing to add all the nodes until a route is completed. This method provides extremely poor answers when some nodes cannot be achieved due to limitations on time, fuel, or spatial restrictions. Ideally, another algorithm that can be provided a budget in which to solve the problem should be used. When the cost budget is depleted, the final set of points is a reasonable subset that considers each node and its place in the total route.

\subsubsection{The Insert Furthest Algorithm}

The 'insert furthest' is another simple-to-implement algorithm for providing an initial tour. The basis for the algorithm is to locate the furthest points from the origin first until all nodes have been accounted for which begins by finding the furthest node from the origin. Once that node is located, each link in the current route is cycled through, broken, and the furthest node is inserted where the cost is lowest. This method works since the best tours will form paths that do not wrap back over segments of their tour. By inserting each node at minimal cost, a very good initial route is built up for the optimization algorithm. 


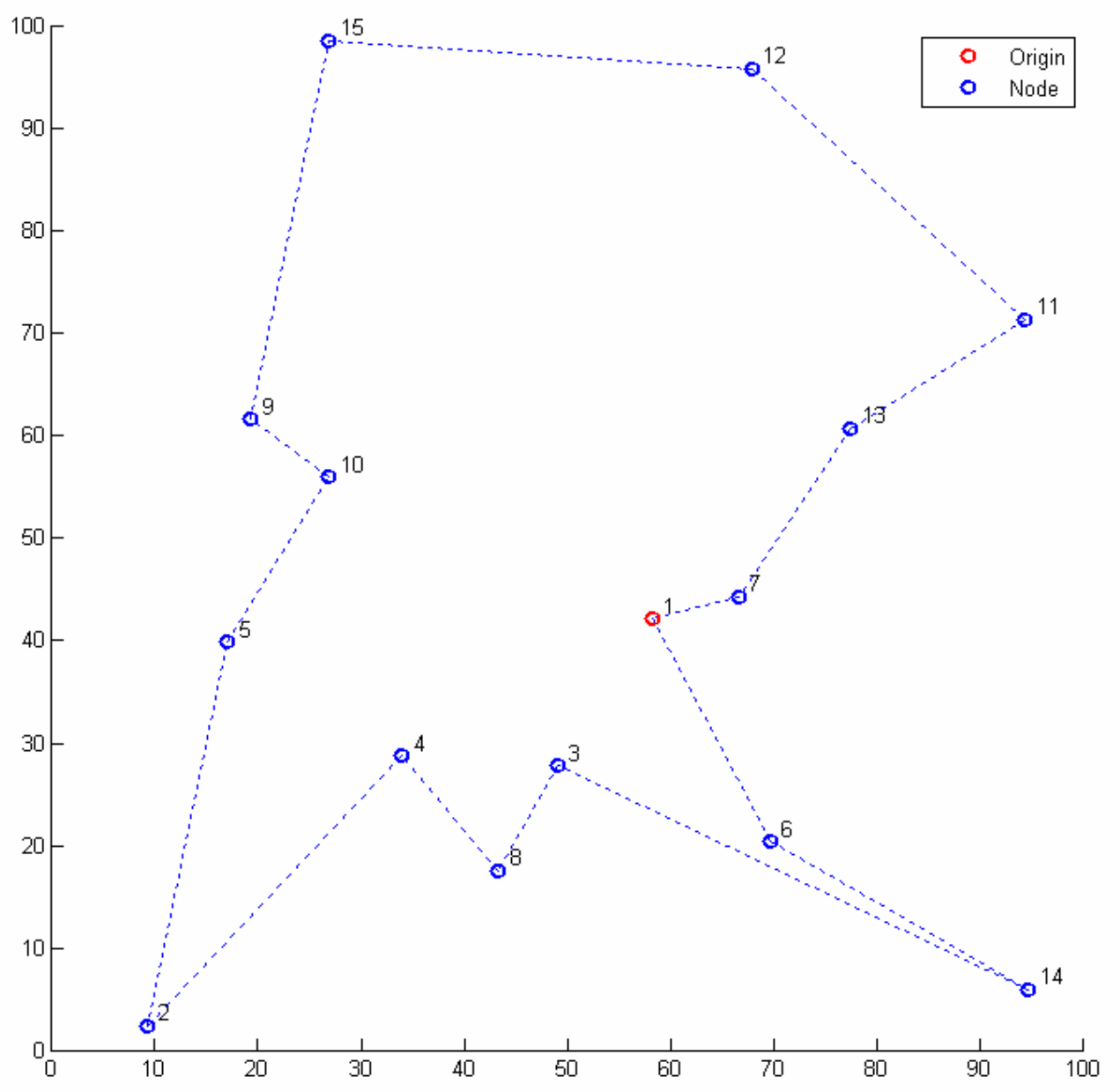

Figure 22: Insert Furthest Algorithm with No Optimization

While the furthest insertion algorithm typically provides good results, the limitations of this method with finite distances and cost budgets are exceptionally severe primarily due to the algorithm making the basic assumption that all points will ultimately be added to the route. When this algorithm is applied with a cost constraint, the algorithm will falter if the distance to and from the furthest point in the set of nodes exceeds the allotted route cost. Even if this node is removed, an attempt is still made to add the most costly node first, immediately exceeding the budget. As a result, this algorithm will only return an unusable route of one node under most circumstances. Implementation of this algorithm in a realistic scenario requires that a subset of nodes be selected first to ensure that all are capable of being met under the allotted cost budget. 
Finally, the achievable nodes are run through the algorithm to create an initial tour with all other points removed.

\subsubsection{The Insert Closest Algorithm}

The insert closest algorithm works essentially the same as the insert furthest method except that the points are added as they radiate out from the origin. To begin, the surrounding nodes are examined to find the cheapest cost from the origin. Once the cheapest addition node is located, the segments in the building tour are searched for the cheapest insertion point. The process is repeated for the next node. For the following node however, the route is then examined to find the cheapest place to add this node. This process is continued until all nodes have been added to the route. 


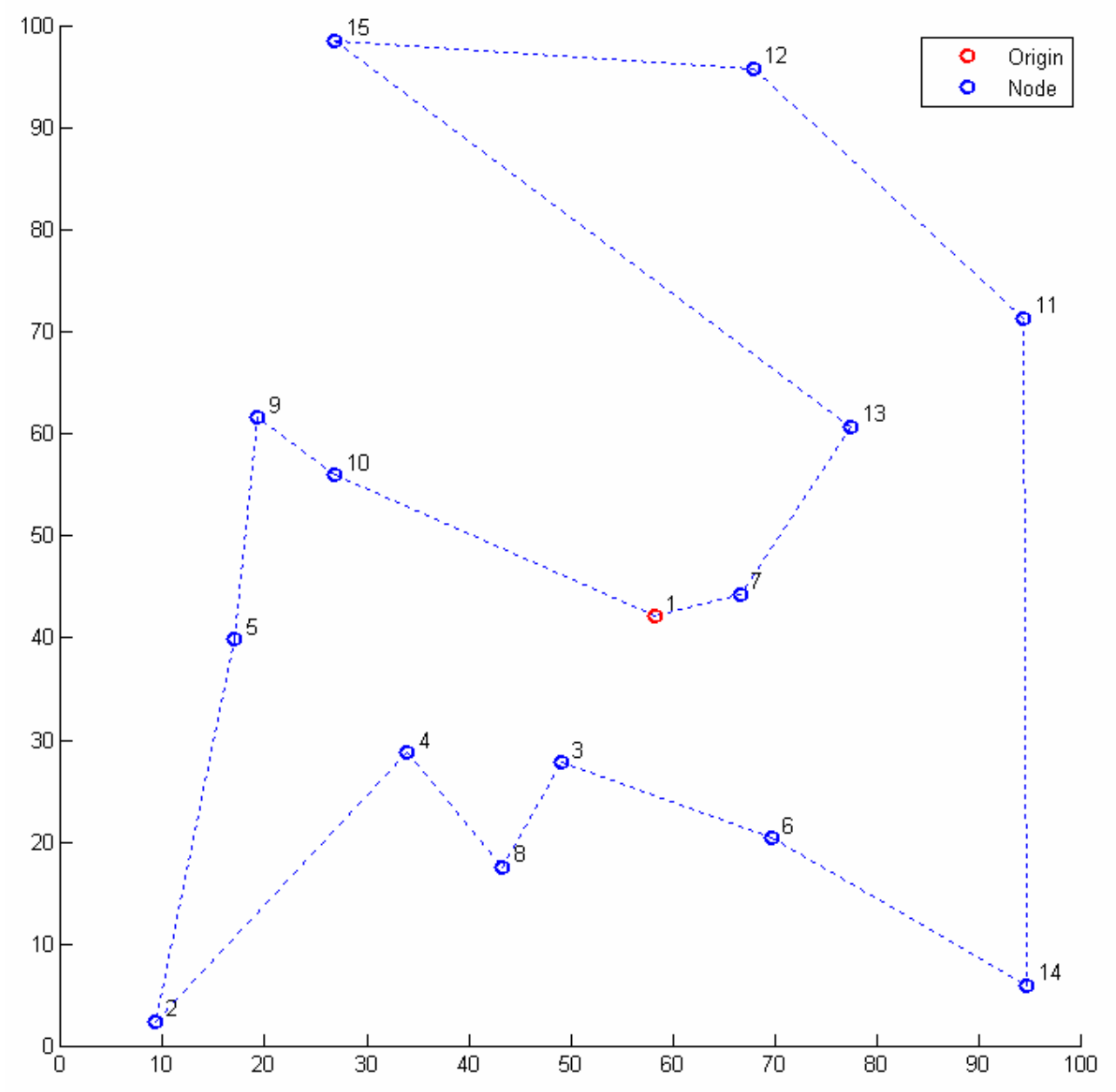

Figure 23: Insert Closest Algorithm with No Optimization

Among the initial route building algorithms, the results for this methodology typically are mediocre and is due to the fact that the algorithm will find local minima where the cost is low but the route is suboptimal. The phenomenon occurs because points are added as they radiate from the origin and the algorithm adds the furthest nodes once the majority of the route has been established. This algorithm, however, is ideal for realistic problems since it builds the route from the origin, ensuring all the selected points can be met. Additionally, if a cost budget is exceeded, a route still can be extracted to meet the required cost. While the route may not be the ideal solution, the algorithm serves as a good starting point and can be utilized effectively as preprocessing. 


\subsubsection{Two-Opt Optimization Algorithm}

The Two-Opt algorithm is a classic example of optimization for the TSP and works by first selecting two links out of the tour. Using these two sets of sequential nodes in the route, the end points are swapped, switching the links, and any cost benefit is then noted. If there is a reduction in the total cost of the route, the switch is finalized. Another set of nodes is then randomly selected and checked with the process repeating until no further optimizations are found.

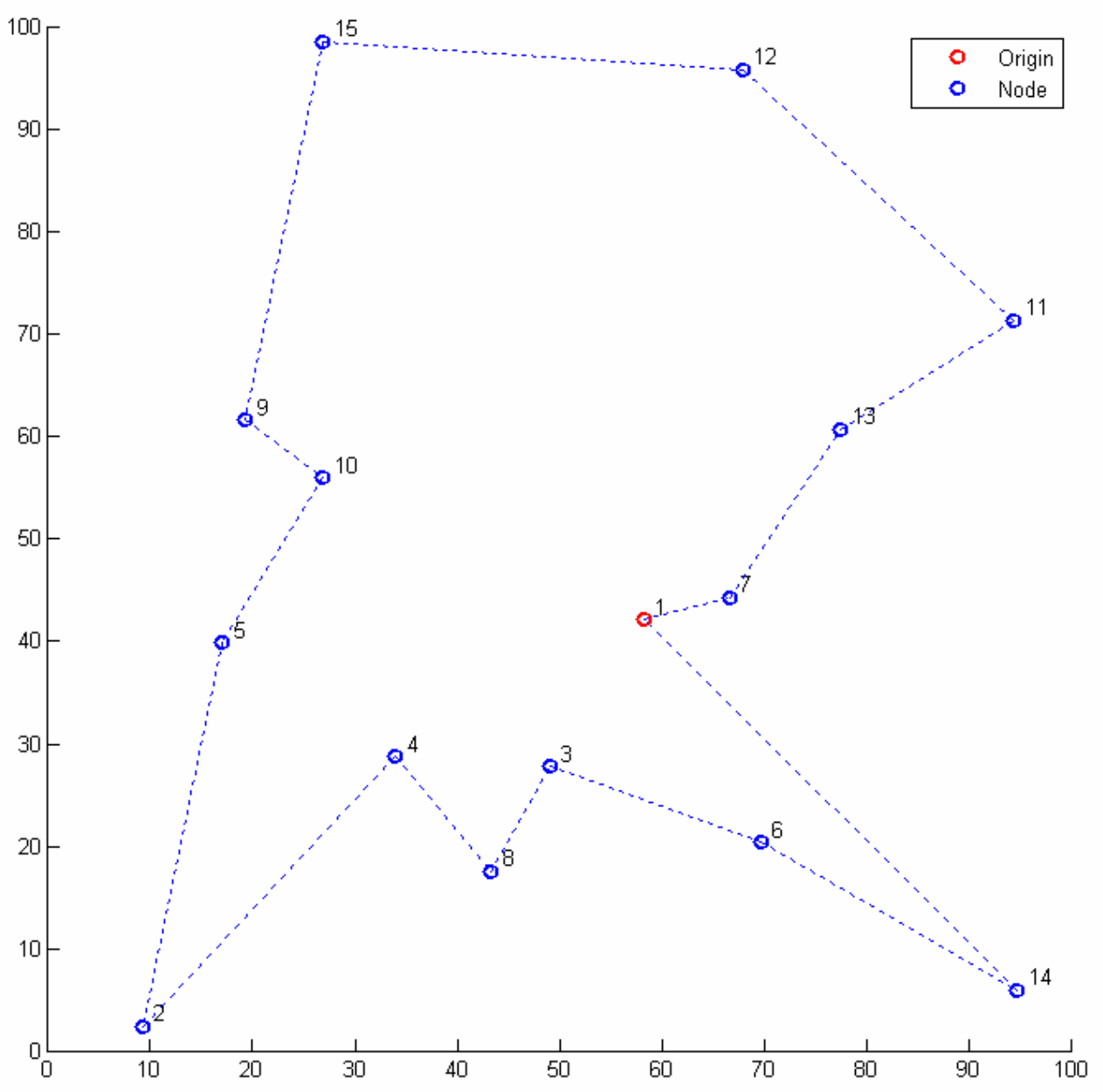

Figure 24: Two-Opt Optimization Algorithm with Random Initial Tour

This routine is particularly suited for aircraft applications since it does not require heavy computational power. Capable of solving smaller problems with an exact solution, the Two-Opt 
algorithm proves to be a favorite for optimization. However, as the number of nodes grows, the probability of obtaining an exact solution decreases and, unfortunately, this is a price paid for limiting the computation cycles and number of passes through the route. While multiple passes can be accommodated, the concept of diminishing returns is a substantial problem for limited onboard aircraft computations. The Two-Opt algorithm poses the best choice for an onboard planner despite obvious limitations with larger problems.

\subsubsection{Three-Opt Optimization Algorithm}

As an extension of the Two-Opt algorithm, the Three-Opt algorithm extracts three links from the current route as opposed to two. All combinations of these links are then searched for a possible decrease in route cost among the six possibilities. Once again, this is accomplished by switching end point nodes for any noted reduction in cost. If any optimization is noted among the subset, the switch is finalized and the next set of nodes is attempted. This algorithm then continues searching through the path sequentially until no optimizations are located. 


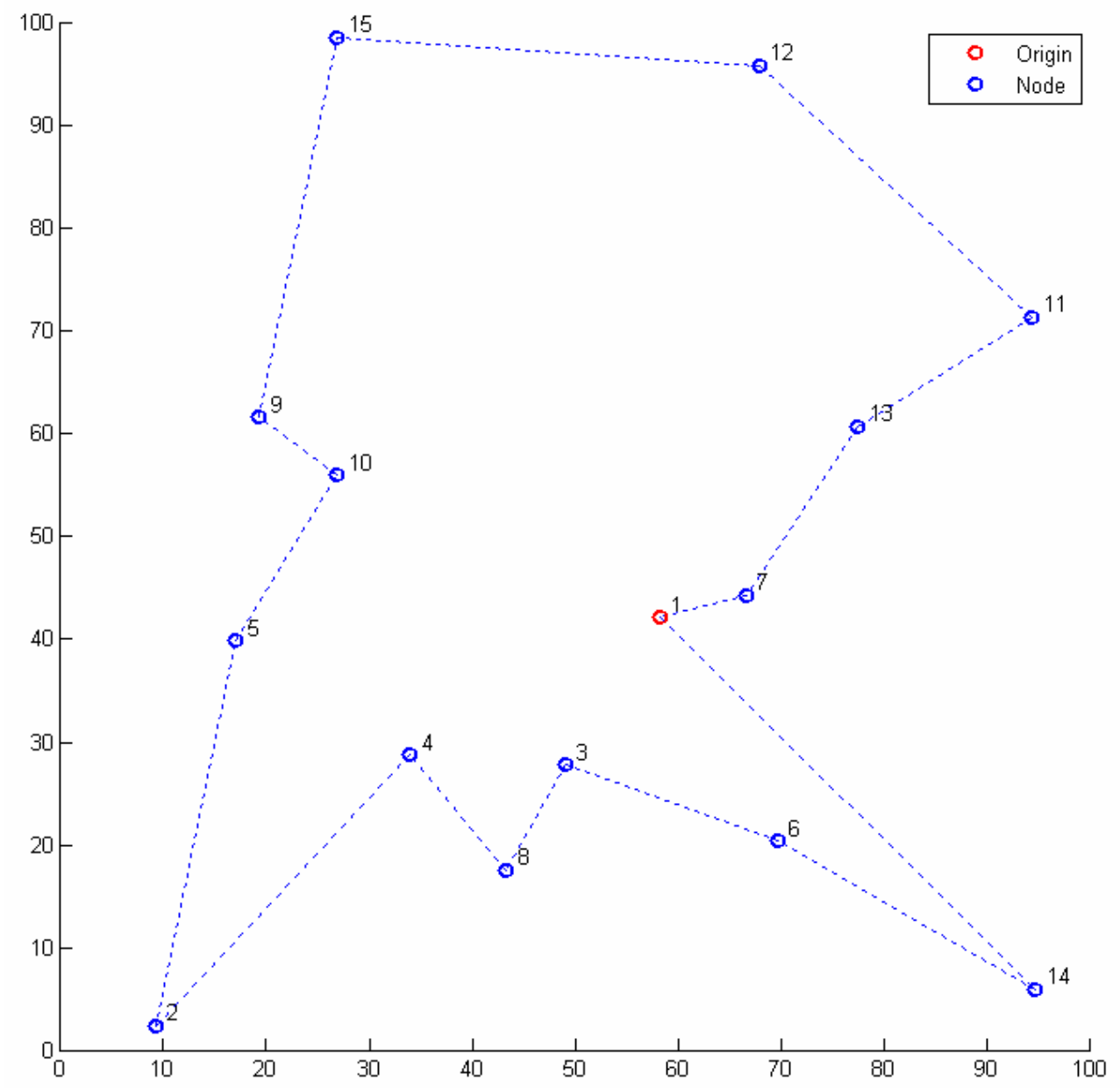

Figure 25: Three-Opt Optimization Algorithm with Random Initial Tour

As expected, the Three-Opt algorithm converged on the same optimal solution as the 2-Opt as they are from the same family of algorithms referred to as the K-Opt. While this is a small problem set for this particular algorithm, the Three-Opt will typically provide better results than the 2-Opt since it explores more combinations in the route. Unfortunately, since more combinations are explored, the computational time grows exponentially as the problem size expands which becomes extremely detrimental when onboard computing is essentially reserved for the flight control system. A lengthy amount of time required before yielding an answer also forms another problem since the information provided may change while the optimization algorithm is working which eventually enters the realm of diminishing returns with larger 
problems since the final tour will take longer to achieve while not providing any substantial gain over more efficient algorithms.

\subsubsection{Algorithm Down-Selection}

When considering the non-linearity of real world problems, it becomes necessary to choose a set of algorithms that is both robust and inexpensive to iterate for a solution. This is especially true when considering realistic problems require limited computational power and where the overall answer can directly impact mission efficiency. Given the aforementioned algorithms, twelve combinations can be created based on the three initial tour algorithms, two optimization routines, and randomly generated sequence as the control case. As shown below in Table 3, the results demonstrate that an optimization routine consistently provided better results with both the 2-Opt and 3-Opt managing to find an optimal solution. The insert closest algorithm used with the 2-Opt failed to find the optimal path and remained at the local minima. The results however are inconclusive and represent a relatively small sample size.

Table 3: Computation Results for Sample 15 Node TSP Problem

\begin{tabular}{|c|c|c|c|c|}
\hline \multirow{2}{*}{\multicolumn{2}{|c|}{ Final Tour Results }} & \multicolumn{3}{|c|}{ Optimization Algorithm } \\
\hline & & None / Random & Two-Opt & Three-Ont \\
\hline \multirow{4}{*}{ 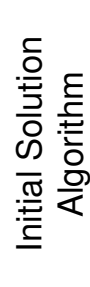 } & None / Random & 700.35 & 394.93 & 394.93 \\
\hline & Greedy & 438.05 & 394.93 & 394.93 \\
\hline & Insert Furthest & 396.82 & 394.93 & 394.93 \\
\hline & Insert Closest & 451.76 & 451.76 & 394.93 \\
\hline
\end{tabular}

Since a concise analysis is required to select the algorithms necessary for the HALE UAV, a series of Monte Carlo simulations is required to determine the most efficient algorithm. For this analysis, larger problem sets must have been provided and each combination to determine the 
best solution. In the analysis, problem sets of twenty, fifty, and one hundred nodes were created with the same normal distribution between 0 and 100 as the sample tour problem presented. In addition, each case was run twenty times to ensure the results obtained from the algorithm were consistent. For each of these cases, the resulting tour cost was recorded along with the computational time for each algorithm, or set of algorithms, to execute and provide their final results. At the end, the results for the eleven combinations, without the randomly generated control case, in each problem set averaged to provide a baseline value from which to gauge comparative improvement.

Table 4: TSP Solution Data for 20 Nodes and 20 Simulations

\begin{tabular}{|c|c|c|c|c|c|c|}
\hline & & \multicolumn{5}{|c|}{20 Node Problem Size } \\
\hline \multicolumn{2}{|c|}{ Algorithm } & $\begin{array}{c}\text { Cost } \\
\text { Average }\end{array}$ & $\begin{array}{c}\text { Percent } \\
\text { Improvement }\end{array}$ & $\begin{array}{c}\text { Time } \\
\text { Average }\end{array}$ & $\begin{array}{c}\text { Cost } \\
\text { Variance }\end{array}$ & $\begin{array}{c}\text { Time } \\
\text { Variance }\end{array}$ \\
\hline \multirow{4}{*}{ None } & \multirow{4}{*}{$\begin{array}{c}\text { Random } \\
\text { Greedy } \\
\text { Furthest } \\
\text { Closest }\end{array}$} & 1019.75 & $0.00 \%$ & 0.0000 & 98.77 & 0.0000 \\
\hline & & 435.65 & $57.28 \%$ & 0.0007 & 59.69 & 0.0003 \\
\hline & & 383.48 & $62.39 \%$ & 0.0018 & 43.17 & 0.0036 \\
\hline & & 426.43 & $58.18 \%$ & 0.0016 & 48.05 & 0.0031 \\
\hline \multirow{4}{*}{ 2-Opt } & \multirow{4}{*}{$\begin{array}{c}\text { Random } \\
\text { Greedy } \\
\text { Furthest } \\
\text { Closest } \\
\end{array}$} & 384.40 & $62.30 \%$ & 0.0036 & 39.89 & 0.0040 \\
\hline & & 377.71 & $62.96 \%$ & 0.0022 & 40.03 & 0.0033 \\
\hline & & 381.47 & $62.59 \%$ & 0.0014 & 43.25 & 0.0002 \\
\hline & & 382.57 & $62.48 \%$ & 0.0016 & 42.66 & 0.0005 \\
\hline \multirow{4}{*}{ 3-Opt } & \multirow{4}{*}{$\begin{array}{c}\text { Random } \\
\text { Greedy } \\
\text { Furthest } \\
\text { Closest }\end{array}$} & 376.08 & $63.12 \%$ & 0.9434 & 39.72 & 0.1463 \\
\hline & & 375.58 & $63.17 \%$ & 0.3346 & 39.72 & 0.1055 \\
\hline & & 377.49 & $62.98 \%$ & 0.1904 & 42.10 & 0.0991 \\
\hline & & 377.03 & $63.03 \%$ & 0.3422 & 40.02 & 0.1520 \\
\hline
\end{tabular}


Table 5: TSP Solution Data for 50 Nodes and 20 Simulations

\begin{tabular}{|c|c|c|c|c|c|c|}
\multicolumn{2}{c|}{} & \multicolumn{5}{c|}{50 Node Problem Size } \\
\hline \multirow{2}{*}{ Algorithm } & $\begin{array}{c}\text { Cost } \\
\text { Average }\end{array}$ & $\begin{array}{c}\text { Percent } \\
\text { Improvement }\end{array}$ & $\begin{array}{c}\text { Time } \\
\text { Average }\end{array}$ & $\begin{array}{c}\text { Cost } \\
\text { Variance }\end{array}$ & $\begin{array}{c}\text { Time } \\
\text { Variance }\end{array}$ \\
\hline \multirow{3}{*}{ None } & Random & 2637.11 & $0.00 \%$ & 0.0000 & 219.53 & 0.0000 \\
\cline { 3 - 7 } & Greedy & 698.17 & $73.53 \%$ & 0.0021 & 56.01 & 0.0003 \\
\cline { 3 - 7 } & Furthest & 600.09 & $77.24 \%$ & 0.0058 & 30.76 & 0.0050 \\
\cline { 3 - 7 } & Closest & 685.68 & $74.00 \%$ & 0.0039 & 44.77 & 0.0030 \\
\hline \multirow{3}{*}{ 2-Opt } & Random & 598.32 & $77.31 \%$ & 0.0397 & 30.19 & 0.0051 \\
\cline { 3 - 7 } & Greedy & 594.17 & $77.47 \%$ & 0.0120 & 28.11 & 0.0058 \\
\cline { 3 - 7 } & Furthest & 594.65 & $77.45 \%$ & 0.0085 & 26.53 & 0.0057 \\
\cline { 3 - 7 } & Closest & 618.30 & $76.55 \%$ & 0.0109 & 34.85 & 0.0031 \\
\hline \multirow{3}{*}{$3-O p t$} & Random & 582.01 & $77.93 \%$ & 47.8964 & 27.42 & 4.6169 \\
\cline { 3 - 7 } & Greedy & 575.31 & $78.18 \%$ & 13.4109 & 25.30 & 3.7206 \\
\cline { 3 - 7 } & Furthest & 581.89 & $77.93 \%$ & 7.0223 & 27.21 & 2.8050 \\
\cline { 3 - 7 } & Closest & 580.62 & $77.98 \%$ & 16.7954 & 26.75 & 5.2046 \\
\hline
\end{tabular}

Table 6: TSP Solution Data for 100 Nodes and 20 Simulations

\begin{tabular}{|c|c|c|c|c|c|c|}
\hline & & \multicolumn{5}{|c|}{100 Node Problem Size } \\
\hline \multicolumn{2}{|c|}{ Algorithm } & $\begin{array}{c}\text { Cost } \\
\text { Average }\end{array}$ & $\begin{array}{c}\text { Percent } \\
\text { Improvement }\end{array}$ & $\begin{array}{c}\text { Time } \\
\text { Average }\end{array}$ & $\begin{array}{c}\text { Cost } \\
\text { Variance }\end{array}$ & $\begin{array}{c}\text { Time } \\
\text { Variance }\end{array}$ \\
\hline \multirow{4}{*}{ None } & \multirow{4}{*}{$\begin{array}{c}\text { Random } \\
\text { Greedy } \\
\text { Furthest } \\
\text { Closest }\end{array}$} & 5215.89 & $0.00 \%$ & 0.0000 & 261.18 & 0.0000 \\
\hline & & 951.11 & $81.77 \%$ & 0.0072 & 44.55 & 0.0042 \\
\hline & & 841.10 & $83.87 \%$ & 0.0154 & 25.11 & 0.0034 \\
\hline & & 957.99 & $81.63 \%$ & 0.0102 & 42.99 & 0.0027 \\
\hline \multirow{4}{*}{ 2-Opt } & \multirow{4}{*}{$\begin{array}{l}\text { Random } \\
\text { Greedy } \\
\text { Furthest } \\
\text { Closest }\end{array}$} & 839.30 & $83.91 \%$ & 0.3255 & 24.82 & 0.0645 \\
\hline & & 811.84 & $84.44 \%$ & 0.0656 & 26.45 & 0.0154 \\
\hline & & 832.17 & $84.05 \%$ & 0.0285 & 25.24 & 0.0084 \\
\hline & & 860.33 & $83.51 \%$ & 0.0661 & 33.62 & 0.0186 \\
\hline \multirow{4}{*}{ 3-Opt } & \multirow{4}{*}{$\begin{array}{l}\text { Random } \\
\text { Greedy } \\
\text { Furthest } \\
\text { Closest }\end{array}$} & 797.66 & $84.71 \%$ & 819.5708 & 23.00 & 52.7231 \\
\hline & & 789.83 & $84.86 \%$ & 194.7399 & 29.15 & 42.9221 \\
\hline & & 803.56 & $84.59 \%$ & 133.4185 & 27.04 & 37.3643 \\
\hline & & 802.37 & $84.62 \%$ & 256.3924 & 18.35 & 44.6832 \\
\hline
\end{tabular}

Based on the results provided in Table 4, Table 5, and Table 6, several conclusions can be drawn from the resulting cost and computational time data. Without optimization, the greedy algorithm would always perform the poorest, followed by the insert closest, and the insert furthest algorithm would perform the best. No matter the size of the problem, the 2-Opt and 3-Opt algorithms always improved the solution with the 3-Opt consistently finding the better solution 
to the traveling salesman problem. Additionally, the 2-Opt and 3-Opt greedy combinations always resulted in better solutions compared to other combinations with that particular algorithm. The last and most important factor is the computational time associated with solving the TSP. While no optimization obviously produces the shortest computational time, the cost associated with adding the 2-Opt algorithm is minimal and produces noticeable benefits. The 3Opt algorithm, however, consistently uses a substantial amount of time to exhaust all of the combinations and only grows exponentially with problem size. With a problem size of one hundred nodes, the difference of a hundredth of a second versus one hundred seconds can be seen as an unnecessary, or even dangerous, time penalty the vehicle must pay for arriving at a solution. The benefit is also noticeable, but hardly worth the minutes of uncertainty, as the vehicle plans a marginally more optimal route.

The most reasonable choice from this analysis becomes the greedy algorithm combined with the 2-Opt optimization routine which provides the best answer among the 2-Opt combinations while minimizing computational time. Additionally, the greedy is capable of containing a problem within a specified cost budget thus allowing non-linear total cost constraint to be invoked on the problem. A reasonable answer is provided over implementing the 2-Opt algorithm alone since the greedy algorithm reduces the final amount of time taken to reach an optimized answer. While the algorithmic problem is organizing the search targets is conclude, the issue of the searching the regions designated by these nodes is presented. 


\subsection{HALE Architecture Search Algorithms}

With the path creation algorithm established, the next priority is devising a search method for the selected area centered at each node. Based on the established wildfire mission requirements, it was deemed that a non-gimbaled multi-spectral line scanner would be the most low cost solution for data collection. For this particular application, two types of line scanners are readily available: across track scanners (whiskbroom) and along track scanners (push broom). Along with determining the sensor, the method of searching the target must be established to utilize the maximum use of the sensor's capabilities without skewing the data due to environmental disturbances which includes being forced to take data during a turn, while pitching the aircraft, or level flight at extreme attitudes.

\subsubsection{HALE Sensor Configuration}

Across track scanners work by rotating, or oscillating, a mirror which sweeps a single cell across the ground as the aircraft flies. Based on the altitude of this aircraft and the instantaneous field of view (IFOV) of the sensor, a resolution for the scanner can be determined. Since the beam is focused on a particular region at any given time, the readings for this type of sensor provide a higher resolution of slight energy differences. However, the tradeoff for this sensitivity is the sensors ability to collect a wide swath of data while maintaining forward airspeed. If the beam size is widened to make up for this sensitivity, a wide IFOV will yield a high signal-to-noise

ratio. ${ }^{27}$ That the method is heavily prone to image distortion as it relies on a single beam. An example of sensor swath profile as the aircraft flies is provided in Figure 26. 


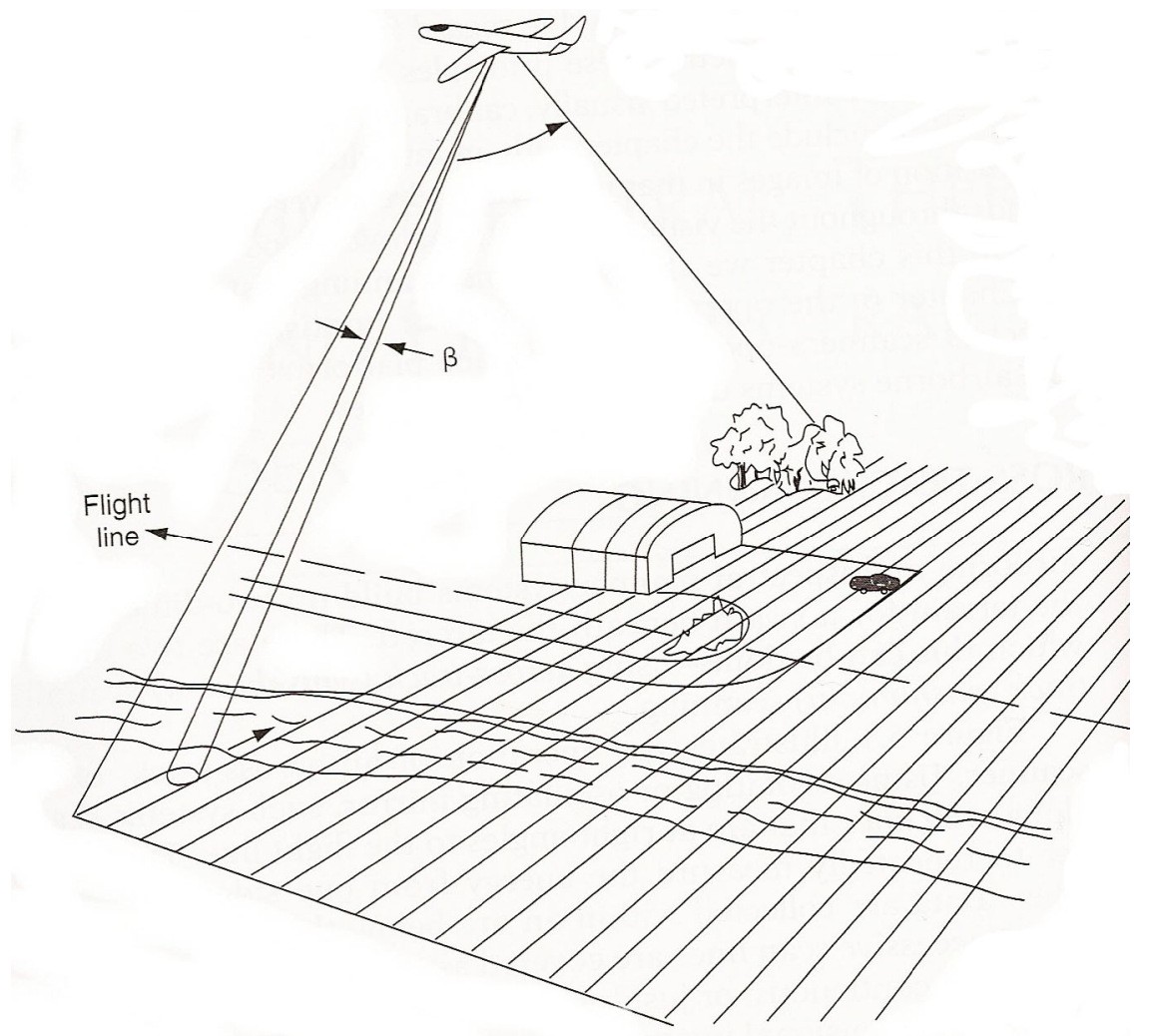

Figure 26: Example of Aircraft with Across Track Scanner ${ }^{27}$

Unlike the across track scanner which uses a scanning mirror, the along track scanner utilizes a linear array detectors. Each one of these array elements is equivalent to a single beam in an across track scanner but in a fixed position, an example of which is shown in Figure 27. This sensor provides a number of benefits over across track scanners including fixed solid state sensors which provide for a smaller, more durable, and more reliable design. The current technology of across track scanners and that the multiple sensors require a longer calibration procedure presents a limitation; at present these sensors are rarely able to detect wavelengths longer than mid-IR. ${ }^{27}$ 


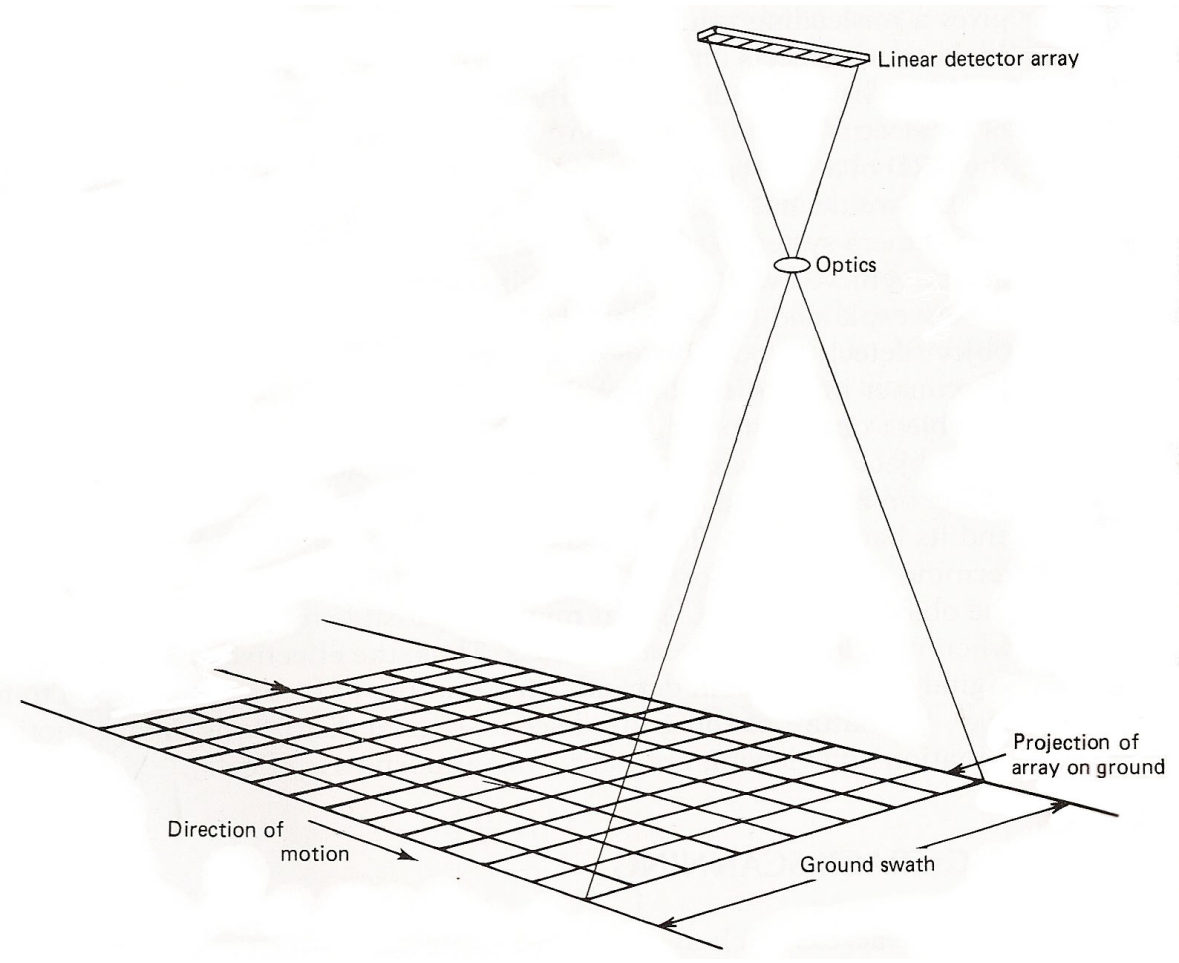

Figure 27: Example of Aircraft with Along Track Scanning Sensor ${ }^{27}$

\subsubsection{Area Search Algorithm}

Based on the down selection and requirements for wildfire missions, across-track scanners are the ideal choice for collecting multispectral data with minimal onboard image processing. However, for this type of data collection and sensor, the aircraft must maintain straight overlapping lines across regions wider than the sensor beam which dictates that a series of lines must be generated as the aircraft crosses back and forth to cover large search regions. Using a generic search region as shown in Figure 28, the generation of such a search pattern becomes a relatively simple routine. 


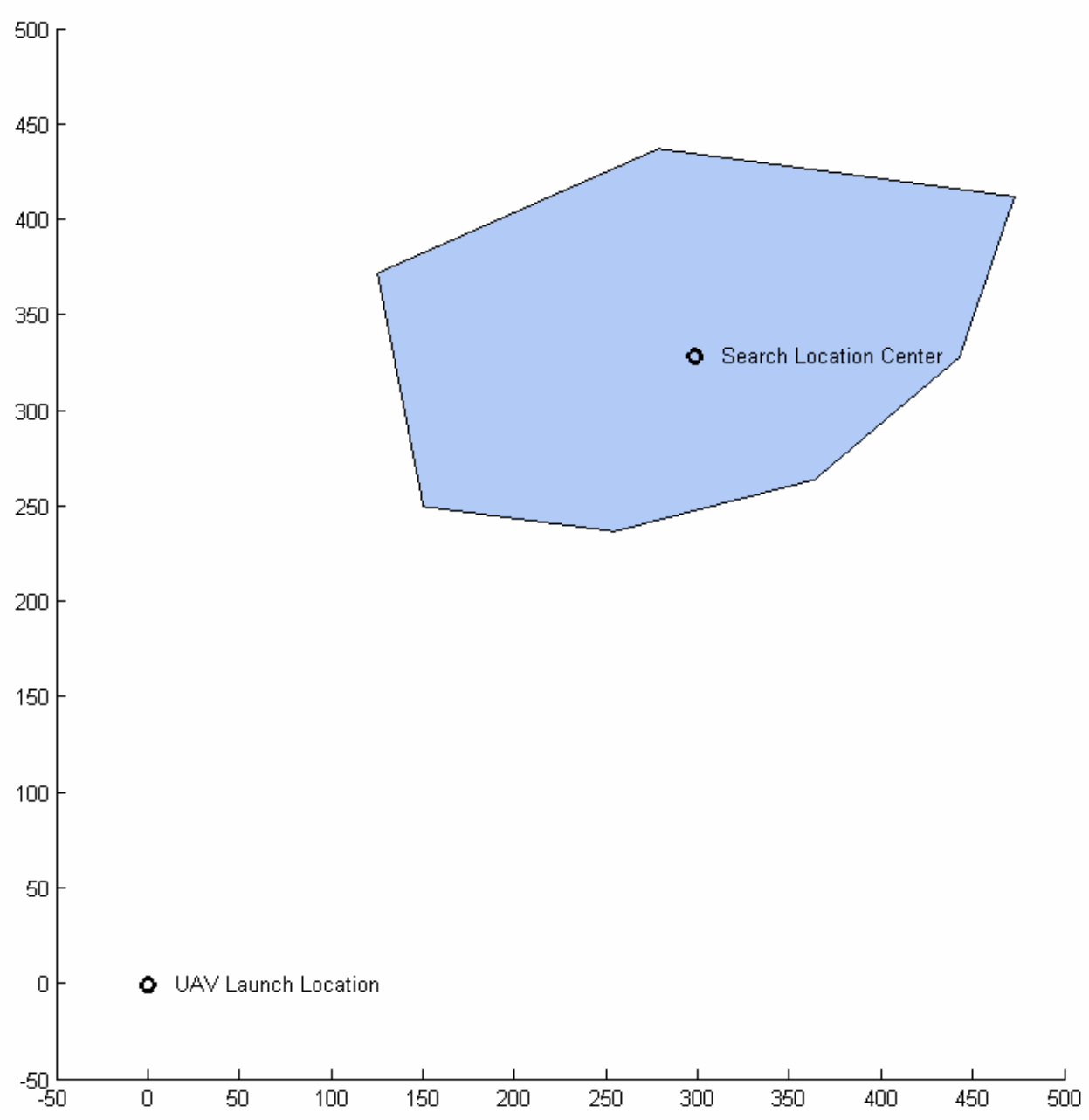

Figure 28: Example of Defined Wildfire Search Location

First, a vector parallel to the data collection lines must be established. This angle can vary depending on the needs for data collection, whether to avoid interference from the sun or optimization based on the search shape geometry. For this example, an assumption is made that the desired vector originates at the current aircraft location to the search area centroid. Each node composing the search region shape is cycled through to locate the furthest and closest points along the vector to the origin. The perpendicular is then constructed, and a similar routine is performed to locate the furthest left and right nodes. Using this information, a bounding rectangle can be constructed around the search region using the external node data. 


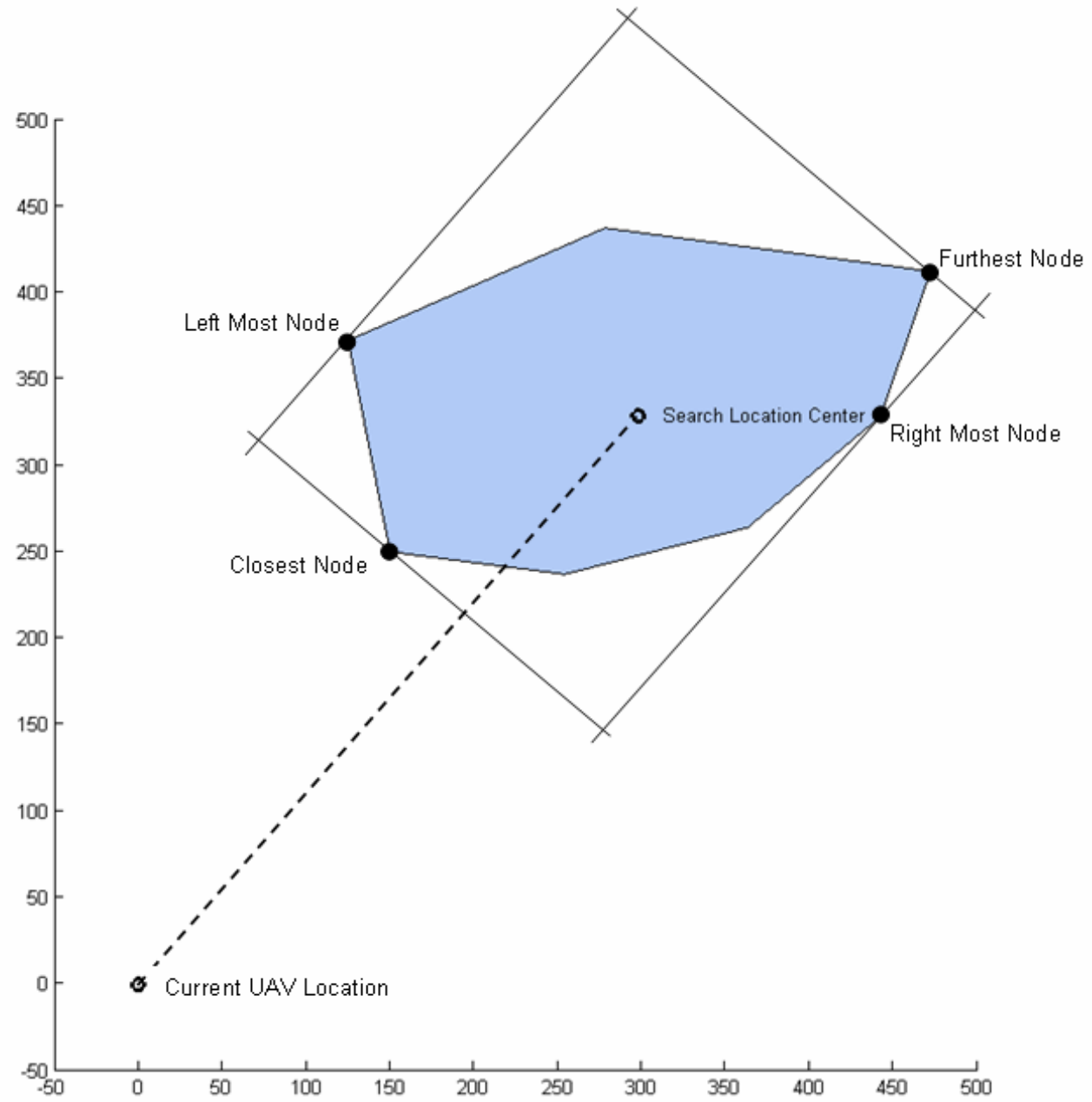

Figure 29: Region with Rectangular Bounding Box for Search Pattern

With a bounding box, this rectangle becomes the framework for the switchback search pattern. The closest edge of the bounding box becomes the inbound flight path. If this is sufficient to cover the search area, the path creation is terminated, and the aircraft moves onto the next target. However, if more area remains to be searched, a path back across the search region is required. Based on the turning radius of the aircraft, a guidance point is then placed in front of this line one full turn radius away which will allow the aircraft to turn onto the line and track the course appropriately. At the end of the track, another guidance point is added to instruct the aircraft to turn and reverse its direction after which, a line is created, parallel to the first but one sensor swath away. This process will continue until the exit criteria of covering the entire area will is 
completed and then proceed to the next target. The final result, depending on the sensor swath width, will provide results similar to those depicted in Figure 30.

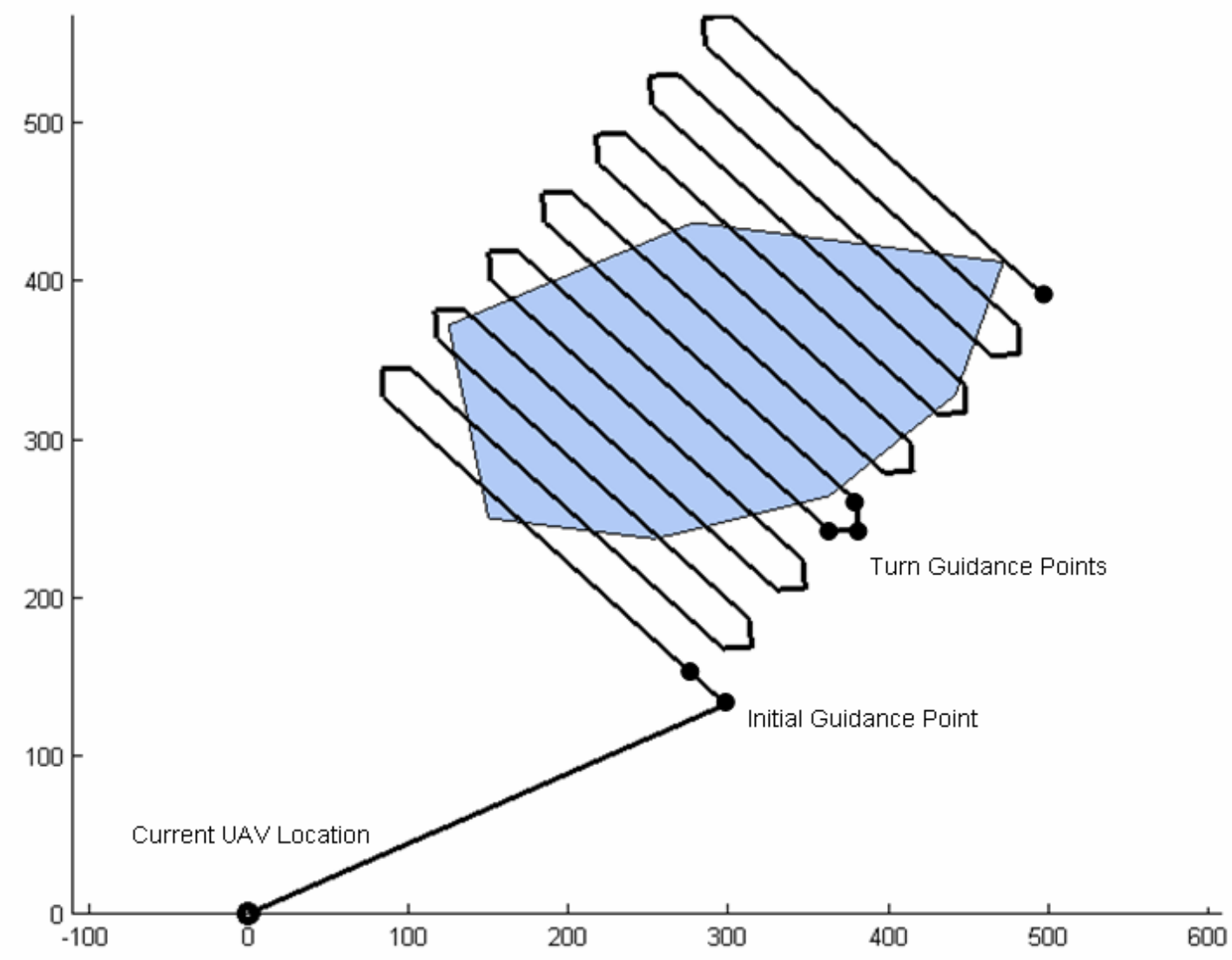

Figure 30: Example Search Pattern for Defined Wildfire Region

Overall, the algorithm proves a simple way of searching a target area by criss-crossing the rectangular bounding box. This methodology was favored over spiral search patterns which skew the data collection and beams concentration toward the edges of the sensor swath. Georectification becomes more difficult as the heading becomes a factor and alignment to previously collected data challenging which is alleviated with straight lines since images can be overlaid with minimal distortion to the path. Linear path tracking is a relatively simple process for the aircraft control system since there is a known heading angle and path which becomes a matter of computing lateral distance off the track and injecting it as an error back into the control system. 
As inferred, the process is very repeatable and allows the control system to compensate for factors such as sun angle and wind when choosing the pattern orientation. In the end, this is a very simple tactic for tracking paths and a standard addition for UAVs with waypoint following.

\subsection{Aircraft and Ground Communications}

Communication is a critical aspect of the parent-child architecture; the details of the objective must be successfully relayed to the other UAV operatives for desired execution of the plan which underlines the significance of determining a format through which information is transferred. In the case of communications software, the most low cost solution is commercial-off-the-shelf (COTS) software that can be maintained by external commercial entities. This readily available communications software is typically referred to as middleware and can vary depending on the code source, supported link types, deployable operating systems and cost.

Middleware is software that provides connectivity between various programs whether it is on the same computer or spread across various machines. When used for inter-machine connectivity, the means of communication can vary depending on what the software supports. For most implementations, Ethernet is the preferred means since it provides adequate bandwidth and IP addresses can be assigned to a variety of hardware despite the type of operating system or source code of the application. As a result, this allows for a superior means for standardizing and linking various agents including other UAVs and ground stations which also reduces development costs for communications architectures since it utilizes an established method for instituting message delivery between entities. 
Among the types of middleware, several variants of communication software exist; the most prominent forms exist in Java, $\mathrm{C}$, and $\mathrm{C}++$ source code. $\mathrm{C}$ and $\mathrm{C}++$ are the only viable options for aircraft systems due to memory allocation and their ability to be implemented on real-time systems. This ability is critical for any software implemented on aircraft hardware since real-time means that the messages will be delivered with the appropriate timing and will not induce latency on other important computations or software functions. Java based middleware is usually centered on web applications since it can be integrated on any operating system. The compatibility tradeoff comes in the form of a higher level coding language making it difficult for hardware interfaces and impossible for real-time operation which leaves a $\mathrm{C}++$ or $\mathrm{C}$ based middleware as the optimal choice for deployment on UAV platforms.

Middleware comes in a variety of implementations since there are numerous applications for such software which not only includes the programming language, but also the method of communication possibly including how and in which the message is relayed. These will become critical decision points for the communications architecture since the UAV parent-child will hinge on how the resources are linked.

\subsubsection{Synchronous versus Asynchronous Communication}

Among the specifications, two types of data relay are available with middleware because of the Ethernet basis: synchronous and asynchronous. For synchronous data shipment, all data is relayed with confirmation and during shipment all other messages are ignored. This is considered a 'blocking' method and, similar to a TCP connection, a direct connection is established to 
ensure message delivery on time. However, this technique is poor when multiple clients require the same information simultaneously or the clients are sending large amounts of data.

Asynchronous communications, on the other hand, do not require on time delivery and can support a higher volume of messages and multiple recipients. As opposed to a regularly timed transmission, this form of communication is noted as event driven and reduces the traffic to only when a data transfer is required. A trade off exists though, mainly because asynchronous messaging systems have the potential of overloading the communication lines with an "everyone for themselves" mentality. Despite this drawback, the primary advantage of asynchronous communication is the publish/subscribe model for disseminating information among multiple agents.

Publish/subscribe communication models are baseline means for having an agent distribute information to multiple resources that require it. Ideal for smaller communications networks, the required data can be published whenever an update is needed on a specific channel. The subscribers are then notified to retrieve the data from the published memory allocation. Two options are available to advise subscribers when new data is available: passive and active.

Passive notification, otherwise referred to as 'polling,' occurs when the subscriber inquires on regular intervals to determine if the publisher has updated the available data and is an undesired technique resulting in extra network traffic that can saturate the network. The polling intervals would need to be quick to ensure that high frequency data is not lost during the intermittent polling periods. The alternative for this is active notification, sometimes referred to as remote 
procedure call, which utilizes a notification from the publisher to activate the subscriber's data collection routine which minimizes unnecessary traffic across the network and serves as an ideal communication model for UAV detection architectures. While the routine does require two exchanges instead of one, it is event driven and only reacts when new information is available which proves ideal for surveillance missions since the higher level architecture does not tax the network and only updates when required.

\subsubsection{Message vs. Object Oriented Communication}

Once the type of communication has been determined, it becomes necessary to define the container, or format, for this information. The methods chosen for data format becomes a critical aspect of any UAV architecture since it must be capable of standardization across all platforms, carry all required data forms, and quickly decipher by an automated network. Two forms of data shipment exist for middleware: message-oriented and object-oriented.

Message-oriented middleware is based on providing event-driven, asynchronous communication in a message based format. This method is typically utilized in inter-personal communication; the content of the message is loosely defined and relies on the application for syntax which equates to a flexible format capable of relaying any content between messaging agents. The limitations surrounding this architecture unfortunately stem from the method's flexibility due to no rigid structure for the data. The overhead of interpreting the information is then required and makes it near impossible for vague human input to translate across to distinct goals. Trying to accomplish this translation would induce potential errors that eliminate the benefits of minimal human interaction. 
Object-oriented middleware provides a rigid framework since all information is defined into data structures. Dynamically sized, it forces the acquired data into a specified format that is easily deciphered by automatic means. While this eliminates relaxed methods of inputting the information, the requirement of filling this rigid data structure allows for a development of costing methods as opposed to arbitrary weighting because of poorly defined information which heavily restricts special conditions, but still allows for expansion since additional fields in the data structures do not disrupt interpretation. Additions to the data require an upgrade of the interpreter to extract only the relevant data.

\subsubsection{Internal HALE Systems Configuration}

Since an object-oriented, publish-subscribe communication model proves the most suitable way to deliver a rigidly structured message between applications, the next phase becomes applying this to the UAV architecture. For the HALE aircraft, much of the functionality described above relies on maintaining communication on board the aircraft which requires a deployment of middleware onboard to interface the UAV inner loop controls and waypoint following routine with payload processed data and adaptive planner. A channel must also be provided so the ground station can supply the desired search locations. Shown below, in Figure 31, is a basic model of how the main ground station interacts with the HALE UAV. The command and control portion deployed by the architecture must be isolated as a payload to the onboard UAV systems. 


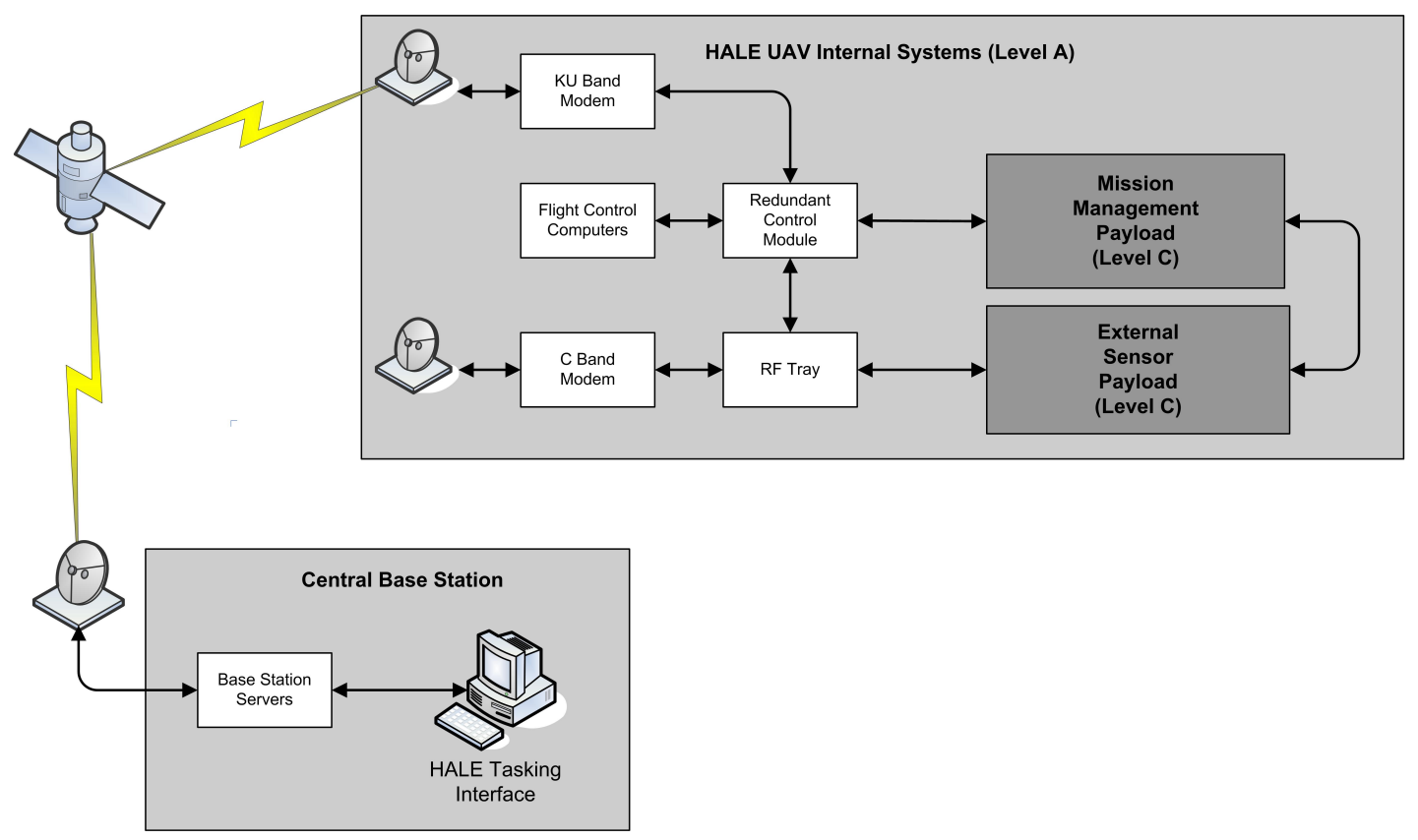

Figure 31: Main Ground Station to HALE UAV Communications

With a basic outline of the onboard communication, the next phase becomes the assignment of this software to a physical location on the aircraft. In order to accomplish this, several key limitations must be observed for the system to be deployed on existing UAV platforms. The most apparent obstacle is testing and cost of implementation for certified software. For FAA flight software certification, software must falls into one of several grades of reliability that ranges from Level A to Level E code. With each of these designations, a letter is then assigned based on the ability to withstand faults or failures. Unfortunately, the substantial cost of acquiring the highest grade of Level A for all software makes it impractical for implementation and the catastrophic fault requirements for Level A essentially rule out COTS software such as middleware since the code is not tested as rigorously.

With considerations on fault tolerance, placement as Level C code for the communications and waypoint following routines proves the ideal solution which dictates that nominal operations are 
reliable enough to guide the aircraft, yet catastrophic failures are avoided by higher reliability code. In essence, the loss of the waypoint following instructions will interrupt the mission, but will not cause the aircraft to deviate into a dangerous situation, reducing the cost drastically and can be treated as an additional layer to the core aircraft flight controls. A separation of these communications and optimization routines must occur from the Level A certified hardware and software that drives the aircraft. The integration of this code and required hardware is then moved to a payload location with a translation module for the commands to the main system. The configuration is expanded in more detail below in Figure 32 which shows the separation between the UAV command and control communications links and the payload driven middleware based information.

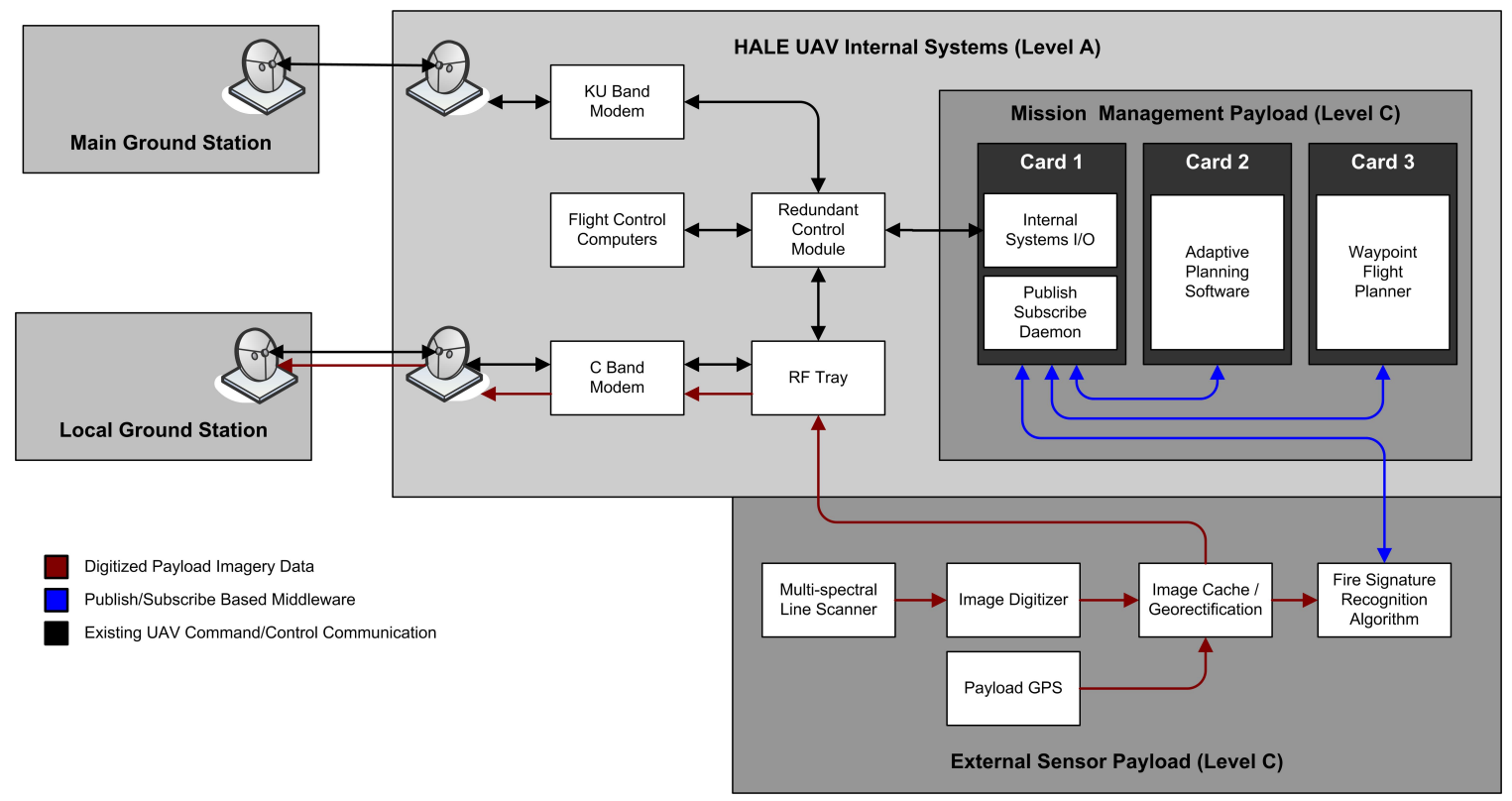

Figure 32: HALE Internal Information Flow Schematic

In both situations, the ground station communication is relayed from the highest and most reliable link. Whether this link is LOS or OTH communications, the payload determines the autonomous instructions. This payload and software will also relay the instructions to the ground 
stations responsible for the LASE UAVs to dictate fire location and reconnaissance results. The onboard HALE systems can register whether a communication link is stable and reliable and communicate the waypoints in an optimized manner for the smaller local assets to respond. Once these commands are accepted at the local ground station level, configuration of the UAVs can be accomplished via a physical connection, cutting out the necessity of having the HALE establish contact with a moving target. Lower cost LASE aircraft can also be used since the means of transferring these commands and determining information are left to the local level.

\subsection{LASE Architecture Search Algorithms}

Once the local ground station has received the information of suspected fire locations, the station then communicates the information to the LASE UAVs. Using a physical connection, the local base station disseminates the information among the available LASE aircraft. Through this direct communication, the base station can determine if the UAV is available and capable of collecting the data at the predetermined coordinates. Once a candidate is selected, the chosen UAV is launched and flies to the potential fire location equipped with a forward looking infrared camera providing a wide FOV of ground below. By means of this camera, the vehicle determines if the fire signature is valid and proceeds to map the perimeter. The aircraft proceeds to track the fire until the entire perimeter has been successfully mapped or the aircraft determines it does not have sufficient fuel to complete the mission. The aircraft will establish the optimal return path and fly this route back to the original launch location.

The ability to deploy the required UAVs locally allows a level of flexibility to utilize any number or type of UAVs for this mission type. The aircraft can be selected on suitability as opposed to 
designing an all purpose aircraft for mission fulfillment. While this does open possibilities, a minimum set of functionality must exist on these aircraft to transform the desired behavior into commands a standard autopilot can execute. Among these basic functions are the abilities of flying generated waypoints, maintaining a coordinated turn and altitude, and provide overall aircraft stability. The development of algorithms can then be laid to allow the vehicle to accomplish this mission element. For simplification, the capabilities required of these algorithms are divided into various modes of operation for the LASE.

\subsubsection{LASE Perimeter Algorithm Modes Overview}

The purpose of the LASE UAV is to fly to a series of predetermined coordinates and then verify the existence of potential wildfires. The algorithms must be capable of determining the vehicle's state at each executed interval which is determined by several triggers that resolve when the aircraft should switch between tasks or modes. These triggers include a fire detection signal, an aircraft fuel status and a waypoint arrival notification. Utilizing these basic event notifications, the behavior of the aircraft can be split into three main modes the vehicle will execute during the course of the mission. The first hinges on flying the aircraft to the potential wildfire coordinate during which the aircraft will alter the heading, using coordinated turns, and maintain a bearing leaving it capable of overflying the target. The second mode occurs when the vehicle notes a fire signature from the sensor and then proceeds to keep the fire perimeter at a predetermined offset while collecting data. The final mode is engaged when the vehicle is either no longer capable of following the fire or has completed following the entire fire perimeter. An outline of the basic mode transitions is shown below in Figure 33. 


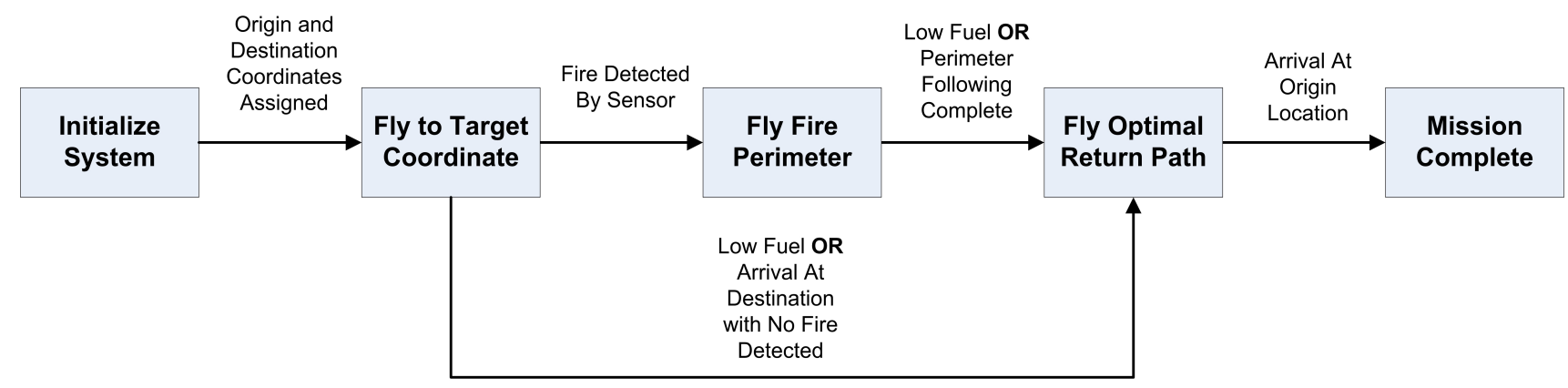

Figure 33: LASE Mission Mode Logic Transitions

The system initialization state encompasses all preliminary tasks such as ensuring the vehicle is capable of undertaking the mission, preflight readiness, uploading of required target information, and takeoff. The vehicle will then take off and spiral upward to the required altitude maintaining a safe margin from any detected fire and allow sufficient sensor visibility. All expected demands of the vehicle thus far are typical of any UAV and are designated as basic functionality with a few additions to interface into the new structure. The algorithms then activate, and the aircraft enters into the first mode of operating flying to the provided coordinate and attempting to verify the fire signature.

\subsubsection{Infrared Camera Sensor Geometry}

For development of this autonomous mode, goals must be outlined beforehand to ensure the vehicle does not enter into dangerous scenarios. The aircraft must be capable of determining the edge of a fire, be capable of resolving this data to the ground plane in order to provide useful information to local resources, and always maintain a safe distance from the fire's edge to keep from crossing directly over the fire. The perimeter tracking behavior must be predictable enough to plan a return route when required. The task then becomes developing an algorithm of 
obtaining each objective. The development of a sensor swath capable of avoiding the fire region while encompassing vehicle limitations is critical.

In order to determine the necessary parameters for the sensor swath, two key factors must be identified: the minimum range and sensor field of view (FOV). The quickest to compute is minimum range, as it depends primarily on the vehicle dynamics, which is computed by considering a worst case scenario where the fire perimeter encompasses the vehicle in straight and level flight. The vehicle must be capable of making a full 180 degree turn within the region of the sensor swath. Assuming a coordinated turn and accounting for a safety margin, the geometry necessary is dictated by the following equations. As demonstrated in the calculations, values are computed at the maximum allowable bank angle capable and the maximum data collection airspeed.

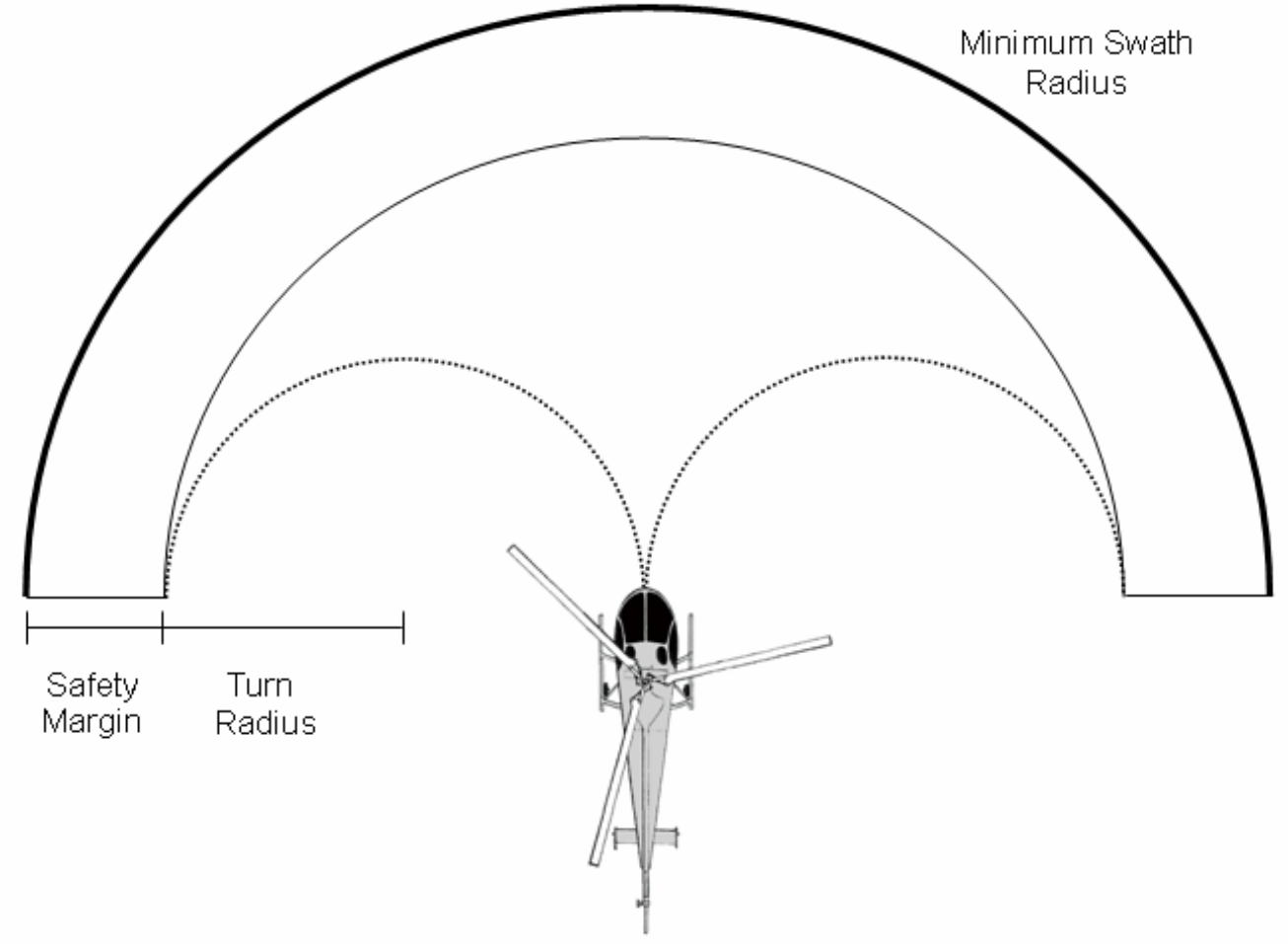

Figure 34: Minimum Aircraft Sensor Swath Radius Dimensions 
V Current aircraft calibrated airspeed (knots)

$\varphi_{\max } \quad$ Aircraft maximum commandable bank angle (degrees)

g Gravitational constant, 32.174 (feet/second ${ }^{2}$ )

$\mathrm{R} \quad$ Coordinated aircraft turn radius (feet)

M Safety margin provided from aircraft distance to fire perimeter (feet)

L Required sensor swath minimum radius (feet)

$\mathrm{R}=\frac{\mathrm{V}^{2}}{\mathrm{~g} \cdot \tan \left(\varphi_{\max }\right)}$

$\mathrm{R}=\frac{(\mathrm{V} \cdot 1.688)^{2}}{\mathrm{~g} \cdot \tan \left(\varphi_{\max } \cdot \frac{\pi}{180}\right)}$

$\mathrm{L}=2 \cdot \mathrm{R}+\mathrm{M}$

For implementation, the safety margin is assumed to be another turn radius which allows the aircraft necessary allotted time to reach maximum bank angle, effectively lengthening the turn radius. Since the rate at which the final bank angle depends on the UAV capability and command authority, a worst case scenario is again assumed. The minimum sensor radius is defined and requires maintenance under any terrain. The shortening of the swath due to pitch and terrain variations is negligible since the vehicle is assumed at a reasonably safe altitude for data collection. The minimum sensor field of view must be accounted for to ensure safe operation. While straight and level flight poses no issue, a banked turn will skew the sensor swath projection on the ground. The maximum commandable bank angle must be accounted for since the sensor vision is reduced while turning away from the fire. Using this bank angle constraint and the desired safety margin, the minimum required sensor field of view is computed by means of the following equations and geometry. 


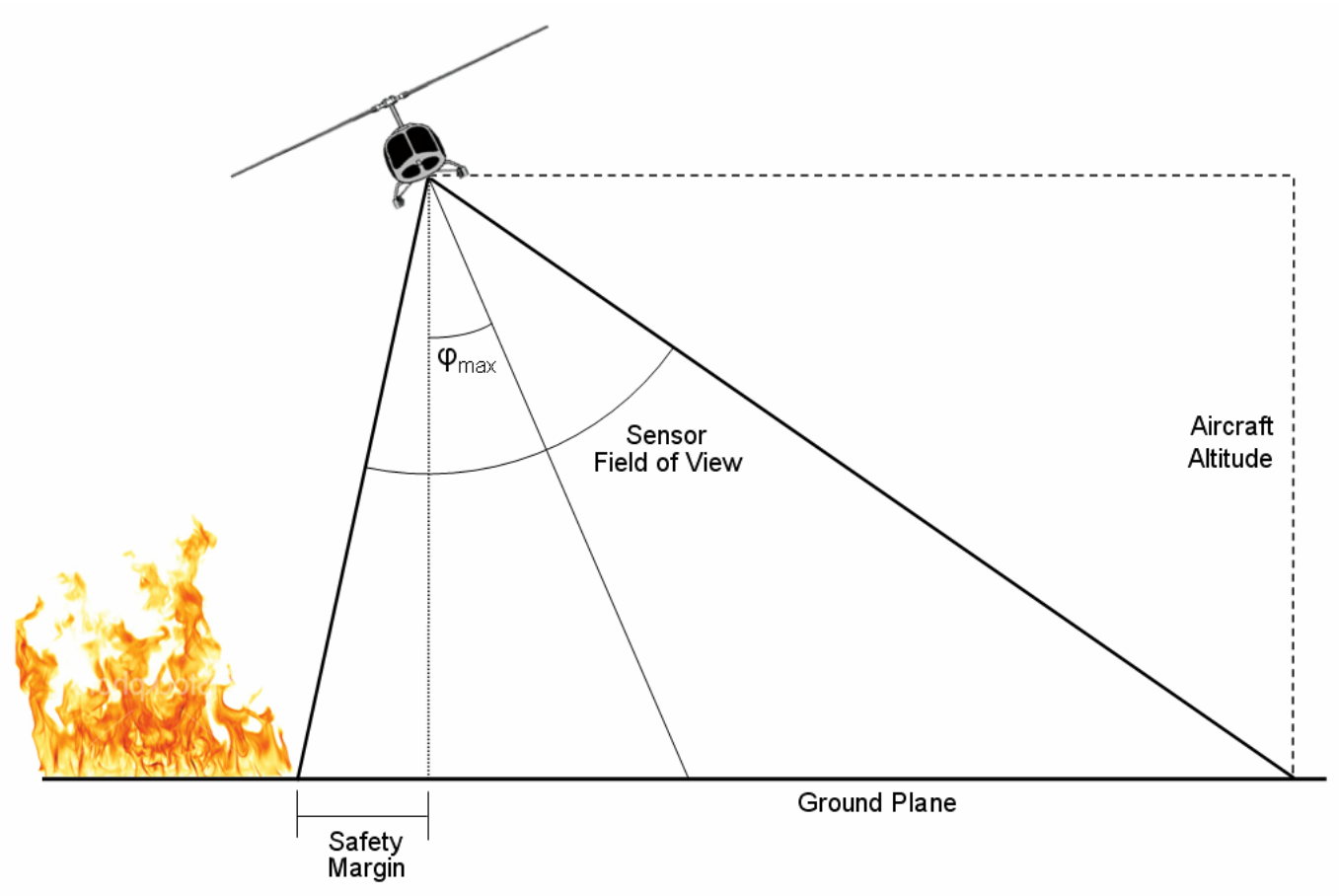

Figure 35: Minimum Sensor Field of View Dimensions

z Minimum aircraft data collection altitude (feet)

$\varphi_{\max } \quad$ Aircraft maximum commandable bank angle (degrees)

M Safety margin provided from aircraft distance to fire perimeter (feet)

FOV Required minimum aircraft sensor field of view (degrees)

$\mathrm{FOV}=2 \cdot\left(\operatorname{atan}\left(\frac{\mathrm{M}}{\mathrm{z}}\right) \cdot \frac{180}{\pi}+\varphi_{\max }\right)$

Utilizing these geometric relationships, values can be computed for sensing the fire perimeter while retaining a relatively safe distance. Preliminary numbers can now be inserted and iterated upon to establish the idea sensor geometry for the aircraft. The sensor vision can then be treated as a slanted cone radiating out from the aircraft to the ground which allows the sensor to fire perimeter interaction to be modeled as a simple line-line intersection with a series of finite length rays emanating from the vehicle nose. The addition of multiple rays also accommodates for the dissipation of sensor resolution as the IR camera views further out. According to the sensor, the 
ray represents the center of an image pixel as the video becomes pixilated while trying to extract detail at further distances. Using the basic dimensioning criteria listed above, the following geometry is assumed for the sensor model. As demonstrated below in Figure 36, the projection has a fixed forward looking alignment from the aircraft nose. The total angle of this arc equates to the sensor field of view, while the radius maps directly to the minimum sensor swath radius defined in Figure 34.

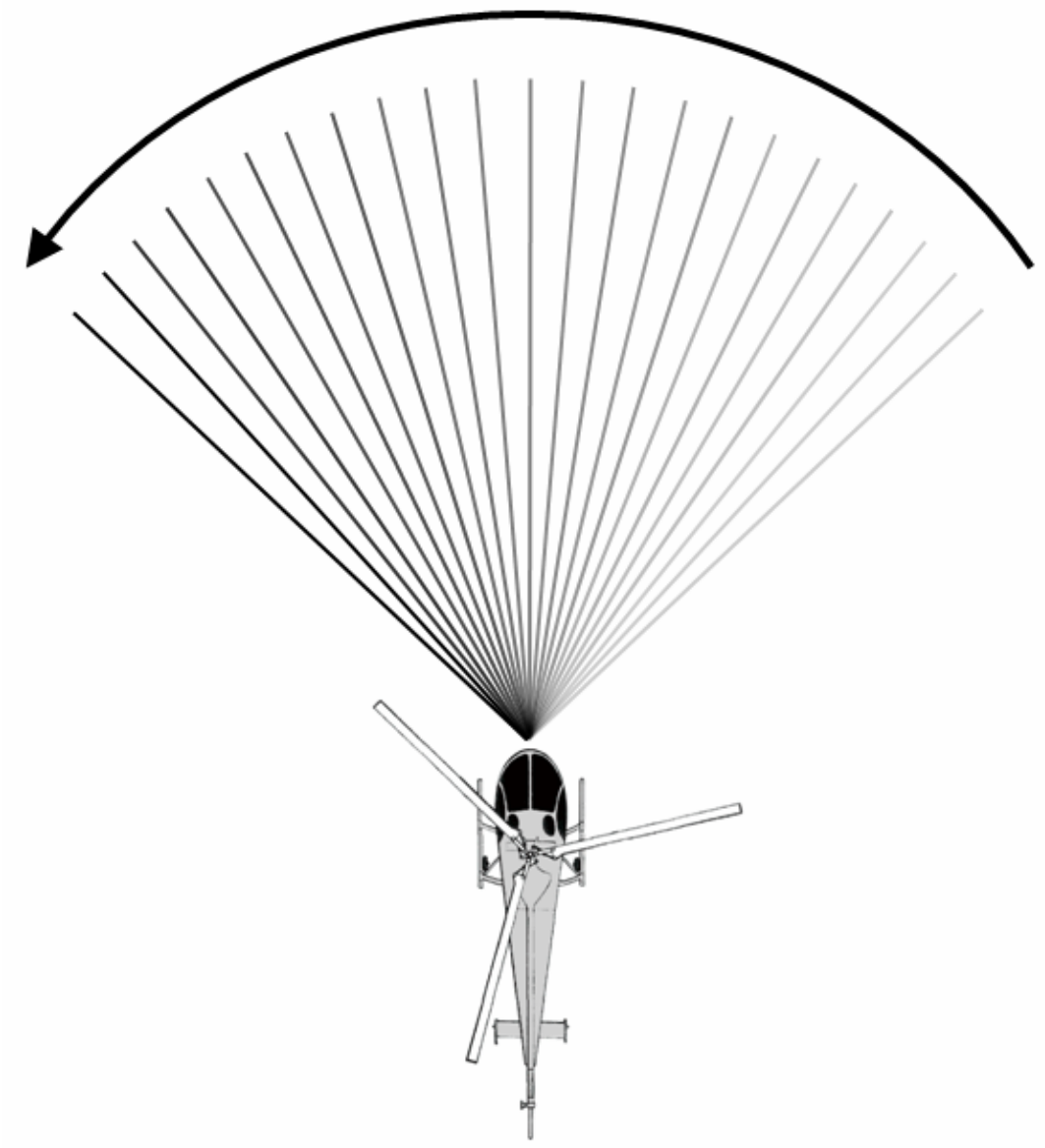

Figure 36: Aircraft Forward Looking IR Camera Sensor Model

With fire perimeter following the most critical mode of operation for the LASE UAV, the aircraft must be capable of reacting to sensed changes in the fire perimeter as it flies. With minimal prior knowledge, the aircraft must be capable of tracking the fire's edge from a safe distance. Operational rules must be required to allow the reactive behavior to provide consistent, 
repeatable results. In this instance, a necessary guideline for behavior is ensuring the aircraft follow the fire perimeter in a counter-clockwise fashion resulting in a number of simplifications in the algorithm as there is no need to support data processing and response in two different directions. This allows the aircraft to immediately turn right after initial fire contact and dictates that the aircraft must always process fire imagery from right to left across the image to determine the angle from the fire and, subsequently, distance away from perimeter.

\subsubsection{Fly-to-Target Coordinate Mode}

As this operational mode hinges on the vehicle's ability to fly to a predetermined waypoint, the algorithm must be capable of determining two critical factors: distance and direction. These are simple quantities to identify however; the difficulty comes in the fact that they must execute quickly onboard a moving aircraft. Regarding the problem of distance, the common method of determining the location of an aircraft is utilizing latitude and longitude provided from GPS which unfortunately is of limited value since this does not provide and clear measure of distance between coordinates nor is the correlation to actual distance consistent in all locations. Degrees of longitude scale to smaller distances as the coordinates move toward the poles. Other techniques for projecting a flat coordinate system onto an elliptical Earth also have their own limitations in representing relative distances.

The flat projection distance computation issue, however, can still be solved by utilizing the same latitude and longitude coordinates provided by GPS. The critical distinction is that, rather than working in absolute latitude and longitude, a difference is established from the launch. This method provides several major benefits over other traditional coordinate systems. First, the 
method creates a local tangent plane centered initially at the vehicle which avoids issues present in predefined plate methods, such as Universal Transverse Mercator, since switching between plates creates an issue with distance computations. Secondly, this becomes a natural fit for LASE vehicles since the aircraft cannot obtain enough distance to be impacted by the curvature of the earth in a flat-earth projection coordinate system. Finally, the conversion is quickly made from available GPS coordinates and can be converted into standard units utilizing only gains and a trigonometric function. The end result of this conversion is a set of coordinates, set on a fixed initial point, with units of feet.

Usage for the conversion is accomplished by first latching onto the current latitude and longitude where the vehicle initializes the algorithm which correlates both the origin and return location for the vehicle, allowing the vehicle to launch at any location and perform the necessary wildfire search functions while remembering the home location. The equations provided below create a coordinate system based on the initial latitude and longitude with positive $x$ as north and positive $y$ as east. This coordinate system is accomplished by first resolving the decimal degrees form of latitude and longitude then to minutes and using the relationship of minutes to nautical miles to result in a distance in feet. 


$$
\begin{array}{cl}
\text { lat }_{0} & \text { Initialized system latitude (decimal degrees) } \\
\operatorname{lon}_{0} & \text { Initialized system longitude (decimal degrees) } \\
\text { lat } & \text { Current vehicle latitude (decimal degrees) } \\
\text { lon } & \text { Current vehicle longitude (decimal degrees) } \\
\mathrm{x}_{\text {north }} & \text { Position change in local tangent plane, positive north (feet) } \\
\mathrm{y}_{\text {east }} & \text { Position change in local tangent plane, positive east (feet) } \\
\mathrm{x}_{\text {north }}=\left({\text { lat } \left.-\operatorname{lat}_{0}\right) \cdot 60 \cdot 6076.11549}^{\mathrm{y}_{\text {east }}=\left(\operatorname{lon}_{-} \operatorname{lon}_{0}\right) \cdot 60 \cdot 6076.11549 \cdot \cos (\operatorname{lat} \cdot \pi / 180}\right)
\end{array}
$$

With the coordinate system established, the proceeding task falls to tracking and flying toward the target. Unfortunately, matching the heading of the vehicle to the target location is insufficient due to smaller UAVs being susceptible to disturbances and crosswinds. As the vehicle can slip to the left or right despite pointing directly at the target. The resolution is not just to close on the orientation of the aircraft nose, but also account for the aircraft trajectory which is accomplished by determining the aircraft's velocity in relation to the ground plane. These quantities are typically referred to as the longitudinal and lateral groundspeeds. Two methodologies exist for obtaining these values, both hinged on transformations from GPS and IMU data.

The first methodology provides the required groundspeeds by differentiating the position change in the North-East coordinate frame. Using the position data provided from GPS and the above coordinate system, the position rate of change is then divided by the update rate. This method assumes an average acceleration in between information updates. The velocities will only have a relatively small error for slower travelling vehicles as the update rate for GPS is on the order of 5 Hz. 
$\mathrm{X}_{\text {north }} \quad$ Position change in local tangent plane, positive north (feet)

$\mathrm{y}_{\text {east }} \quad$ Position change in local tangent plane, positive east (feet)

$\mathrm{V}_{\text {north }} \quad$ Velocity in local tangent plane, positive east (feet)

$\mathrm{v}_{\text {east }} \quad$ Velocity in local tangent plane, positive east (feet)

dt Sample time increment (seconds)

$\mathrm{v}_{\text {north }}=\frac{\Delta\left(\mathrm{x}_{\text {north }}\right)}{\mathrm{dt}}$

$\mathrm{v}_{\text {east }}=\frac{\Delta\left(\mathrm{y}_{\text {east }}\right)}{\mathrm{dt}}$

Once the velocities are established, the quantities are rotated using a standard rotation matrix. Heading to true north is a known quantity for navigation meaning a counterclockwise rotation matrix is induced using a negative value to reorient the coordinate system. Since the aircraft heading is the angle between north and the vehicle nose, the new coordinate system establishes velocities with positive $x$ out the nose and positive $y$ out the right wing from the center of gravity.

$\psi \quad$ Aircraft heading angle from true north (degrees)

$\mathrm{v}_{\text {lon }} \quad$ Longitudinal groundspeed, positive out the aircraft nose (feet/sec)

$\mathrm{v}_{\text {lat }} \quad$ Lateral groundspeed, positive out the aircraft right wing (feet/sec)

$\left[\begin{array}{c}\mathrm{v}_{\text {lon }} \\ \mathrm{v}_{\text {lat }}\end{array}\right]=\left[\begin{array}{cc}\cos (-\psi) & -\sin (-\psi) \\ \sin (-\psi) & \cos (-\psi)\end{array}\right] \cdot\left[\begin{array}{c}\mathrm{v}_{\text {north }} \\ \mathrm{v}_{\text {east }}\end{array}\right]=\left[\begin{array}{cc}\cos (\psi) & \sin (\psi) \\ -\sin (\psi) & \cos (\psi)\end{array}\right] \cdot\left[\begin{array}{c}\mathrm{v}_{\text {north }} \\ \mathrm{v}_{\text {east }}\end{array}\right]$

Though these acquired groundspeeds are in the proper coordinate frame, the update rate of GPS becomes a serious issue for faster fixed wing aircraft; an assumption of average acceleration will breakdown as the vehicle is buffeted by crosswinds and disturbances. The rate of travel can be fast enough to prevent the aircraft guidance system from closing on the vehicle's trajectory toward the target resulting in an alternative method being required for synthesizing 
groundspeeds. The next solution comes from the standard practice of tying inertial guidance IMUs to GPS and determining desired vehicle quantities which is completed using a Kalman filter that uses all available sensor information to estimate the aircraft states and position at a higher frequency. As a byproduct of this state estimation, the body velocities are determined for the aircraft. These velocities are then transformed to the local tangent plane and oriented along the vehicle nose and right wing to create groundspeeds. Using Euler angles for the transformation, also provided by the IMU, the following equation provides the necessary resultants.

$$
\begin{aligned}
& \text { u Aircraft longitudinal body velocity, positive out nose (feet/sec) } \\
& \text { v Aircraft lateral body velocity, positive out right wing (feet/sec) } \\
& \text { w Aircraft vertical body velocity, positive down (feet/sec) } \\
& \varphi \quad \text { Aircraft Euler roll angle (degrees) } \\
& \theta \quad \text { Aircraft Euler pitch angle (degrees) } \\
& \mathrm{v}_{\text {lon }} \quad \text { Longitudinal groundspeed, positive out the aircraft nose (feet/sec) } \\
& \mathrm{v}_{\text {lat }} \quad \text { Lateral groundspeed, positive out the aircraft right wing (feet/sec) } \\
& {\left[\begin{array}{c}
\mathrm{v}_{\text {lon }} \\
\mathrm{v}_{\text {lat }}
\end{array}\right]=\left[\begin{array}{ccc}
\cos (\theta) & \sin (\varphi) \cdot \sin (\theta) & \cos (\varphi) \cdot \sin (\theta) \\
0 & \cos (\varphi) & -\sin (\varphi)
\end{array}\right] \cdot\left[\begin{array}{c}
\mathrm{u} \\
\mathrm{v} \\
\mathrm{w}
\end{array}\right]}
\end{aligned}
$$

Utilizing the groundspeeds referenced to the local tangent plane, the amount of vehicle drift of can be determined. Any lateral velocity must be eliminated, as it infers the aircraft is slipping off course, by accounting for this in the aircraft's heading. By creating a ratio of the lateral velocity to longitudinal velocity, a track angle is created that accounts for the true trajectory of the aircraft. 
$\mathrm{v}_{\text {lon }} \quad$ Longitudinal groundspeed, positive out the aircraft nose (feet/sec)

$\mathrm{v}_{\text {lat }} \quad$ Lateral groundspeed, positive out the aircraft right wing (feet/sec)

$\psi \quad$ Aircraft Euler heading angle from true north (degrees)

$\alpha \quad$ Aircraft actual track angle including side slip (degrees)

$\alpha=\psi+\operatorname{atan} 2\left(\frac{\mathrm{v}_{\text {lon }}}{\mathrm{v}_{\text {lat }}}\right) \cdot \frac{180}{\pi}$

An angle to the target is created by performing the same inverse tangent operation on the north and east error which angle is then subtracted from the above quantity to provide an error signal between the aircraft's current track verses the required vector. This error is then fed to the flight control system and corrected for large heading adjustments via a coordinated turn. For smaller values, a precision heading change may be difficult to achieve via a turn, and should be corrected using the yaw axis allowing the aircraft to maintain level flight. This combination ultimately allows the vehicle to close on the proper trajectory despite large disturbances or high winds.

$$
\begin{array}{cl}
\text { lat } & \text { Current vehicle latitude (decimal degrees) } \\
\text { lon } & \text { Current vehicle longitude (decimal degrees) } \\
\text { lat }_{\text {dest }} & \text { Destination coordination latitude (decimal degrees) } \\
\operatorname{lon}_{\text {dest }} & \text { Destination coordination longitude (decimal degrees) } \\
\alpha & \text { Aircraft actual track angle including side slip (degrees) } \\
\alpha_{\text {err }} & \text { Aircraft actual track angle error (degrees) } \\
\Delta \mathrm{x}_{\text {north }}=\left(\text { lat }_{\text {dest }}-\text { lat }\right) \cdot 60 \cdot 6076.11549 \\
\Delta \mathrm{y}_{\text {east }}=\left(\operatorname{lon}_{\text {dest }}-\text { lon }\right) \cdot 60 \cdot 6076.11549 \cdot \cos (\text { lat } \cdot \pi / 180) \\
\alpha_{\text {err }}=\operatorname{atan2}\left(\frac{\Delta \mathrm{x}_{\text {north }}}{\Delta \mathrm{y}_{\text {east }}}\right) \cdot \frac{180}{\pi}-\alpha
\end{array}
$$


Using the above as inputs to the LASE UAV controls, the aircraft will fly to the potential fire signature until a waypoint arrival notification is provided by the onboard processing. At this point, if the aircraft has not detected any fire, it will assign the origin location as the new destination. The computation is then repeated as the aircraft returns to the local fire station. However, if a fire signature is determined by the forward looking IR camera, the aircraft will switch modes fire perimeter tracking in order to track the perimeter of the fire.

\subsubsection{Fire Perimeter Tracking Mode}

In real-time, the IR video data is examined from right to left across the image for signs of high temperature discrepancies in the payload module. While in route to the selected destination point, if a fire signature is noted during the data processing, the aircraft will switch into a fire tracking mode. While many UAVs today rely on predetermined coordinates and flight plans, the reactive nature demanded by this changing environment requires a break from the traditional paradigm. The standard technique of handing control over to a human pilot can present multiple problems. A human operator must be designated to oversee the UAV which unfortunately diverts resources away from the primary focus of firefighting. Additionally, the requirements and costs for UAV operation skyrocket when a trained UAV pilot ground station must continuously be at the ready despite long lulls in between missions. For this purpose, an UAV operational mode for this mission must be developed capable of interpreting sensor data and reacting autonomously.

In order to accomplish fire tracking tasks, the processing and interpretation of the fire signature from the sensor swath becomes absolutely critical. As demonstrated with the fire sensor model shown in Figure 36, the video image is scanned from right to left for white hot images appearing 
within the IR camera's instantaneous field of view. If a signature is detected, the aircraft orientation, along with the location of the signature in the image, is recorded by the aircraft. The software then translates the image from the vehicle frame to a reference plane level with the ground.

Through the above noted corrections for the pitch and roll rotations, the relationship between the fire and the current aircraft trajectory can then be established. Since the images are processed continuously throughout time, a constant readout of the fire detection angle is provided. Using a desired contact angle to maintain a consistent distance from the fire perimeter, an error signal can be computed from the detected fire contact angle which is then fed into the roll axis or yaw axis depending on the magnitude of the value. For smaller errors, the yaw axis is used to maintain precision data collection; regarding larger error values, a coordinated turn is initiated up to the maximum desirable bank angle to close the heading error. As the radius of the sensor swath was designed to accommodate a full turn, very little danger is presented to the UAV during these maneuvers.

As shown in Figure 37, the helicopter is depicted as tracking the fire perimeter with the sensor swath arc. As the image processing algorithm travels along the arc, the solid black line represents the ideal contact angle to be maintained by the aircraft. The dashed blue line denotes the actual contact angle determined through the thermal fire signature. In the first image, the aircraft must rotate clockwise to align the detected angle with the desired. Once this point has been determined by the furthest blue dot, the vehicle reacts to the sensor data and makes the appropriate vehicle corrections. In the second depiction, the aircraft once again determines the fire contact angle at 
the edge of the sensor arc and must make a counterclockwise rotation to align the determined contact angle in blue with the desired angle in black leaving the UAV are made capable of tracking the fire perimeter with high frequency sensor data and ensuring consistent corrections to maintain a desired fire track angle.

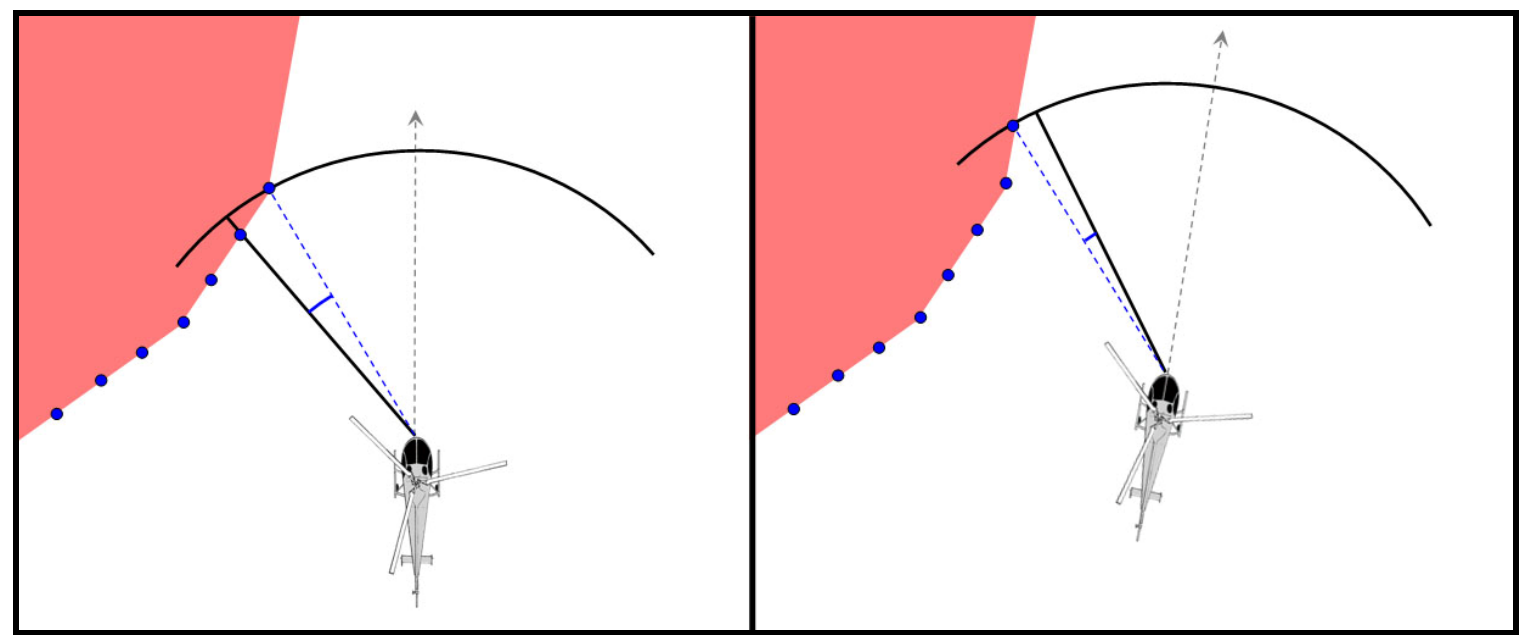

Figure 37: Aircraft Maneuvering to Follow Fire Perimeter

Using the tracking behavior, the vehicle is capable of reacting to, and encompassing the perimeter of, any arbitrarily shaped fire. The algorithm can do so reactively while maintaining the required distance from the fire's perimeter which this completes the objective of being able to follow and map the outline of a generic wildfire. During the perimeter tracking flight, two exit conditions exist that will allow the vehicle to stop and return back to the designated home base or launch point: (1) the fire perimeter has been fully mapped by the UAV circumscribing it with the aircraft's flight path and (2) the vehicle estimates that it has only enough fuel to retrace its previous trajectory and return home via an optimized route. During both conditions, the vehicle will invoke the final algorithm that tracks waypoints returning back to the home locations. 


\subsubsection{Optimal Return Path Mode}

For the final stage of the algorithm development, a method is required that allows the vehicle to return back to the point of origin, using the acquired fire perimeter data, and while determining an optimal return solution. To accomplish these tasks, a 'rubber band' solution, shown in Figure 38 , is necessary which uses the predetermined fire perimeter points to direct the vehicle around any dangerous areas while also removing unnecessary segments of the course. As a result, the proposed answer to the optimal return path is akin to stretching a string across a region filled with gaps, as depicted below. The consistent counterclockwise rotation around the fire perimeter allows a clockwise rotation to always be utilized back to the origin.

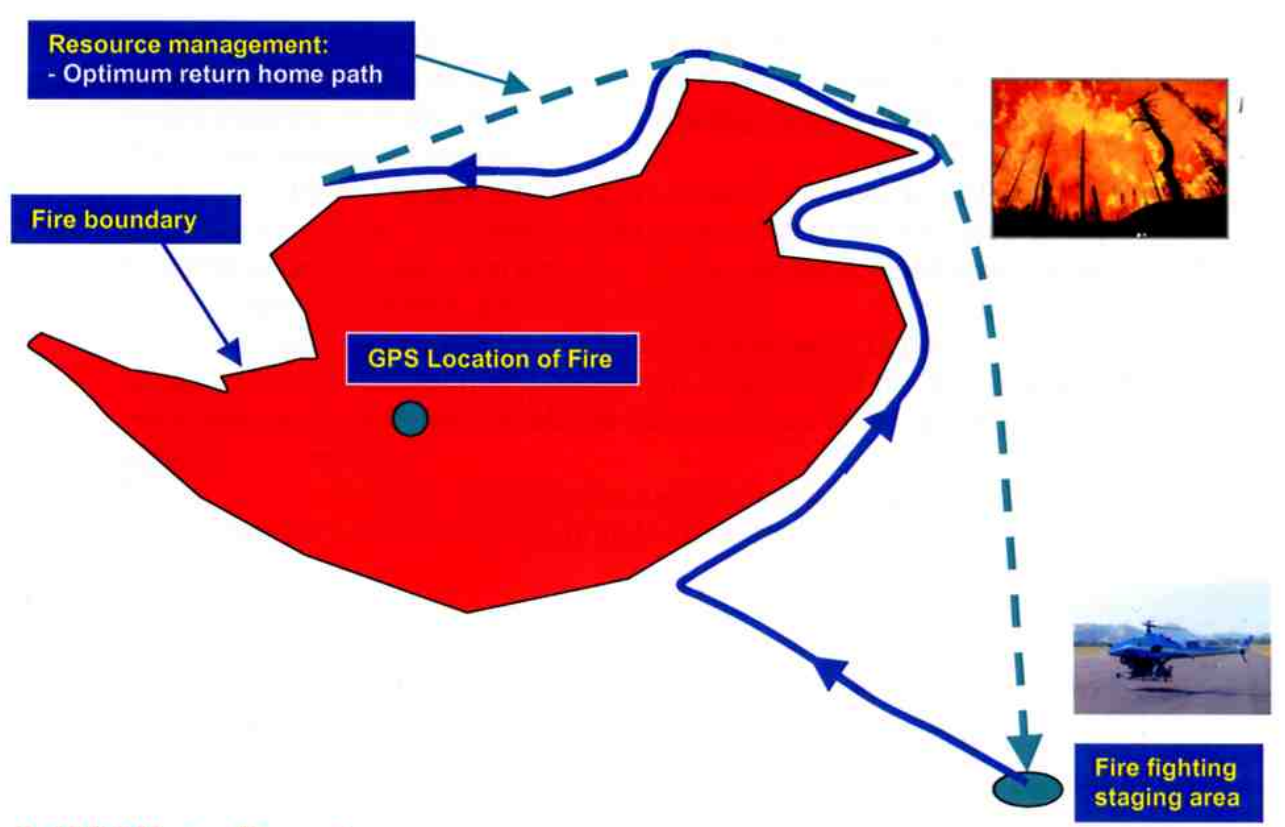

Figure 38: LASE Aircraft Return Path Algorithm Objectives

In order to achieve the proposed solution, the basic answer is provided by a convex hull algorithm which operates by attempting to locate the outermost points in a set of twodimensional or three-dimensional points. When these points are connected, they enclose the remaining coordinates as depicted in the optimized solution above. Though the convex hull 
algorithm is quick, robust, and low-cost computationally to implement, several caveats exist to employing this solution on the LASE UAV. The engagement criterion to enter this mode depends on the available aircraft fuel or whether the aircraft has completed its mission. The convex hull must be computed at each update interval to provide a total distance the vehicle has, and must, travel in order to return to the launch location. The convex hull algorithm must also have at least three points that are not collinear. Finally, the algorithm only returns a series of exterior points from an arbitrary set. The solution returned by the algorithm must then be sorted to ensure the origin was not removed due to enclosure and the series rotation maintains a clockwise rotation back to the origin.

From these constraints, the aircraft is required to identify an instantaneous solution at each sampling interval while detecting a fire perimeter. In order for this to be accomplished, a path is tested against the convex hull algorithm whenever the number of stored indices is greater than or equal to three nodes. Considering the origin and current aircraft location cannot be excluded, the operation is initiated whenever a single perimeter point is added to the listing. The sequence of points is then entered into the convex hull algorithm, and a closed circuit of nodes is returned. A series of checks and modifications must then be executed to ensure that the result returned by the convex hull is a valid sequence, with the proper rotation, and with no important points omitted.

The order of points returned by the convex hull algorithm must be first verified because the algorithm only encloses the submitted points, but does not specify an order of rotation around the fire perimeter. Since the aircraft has specified a counterclockwise flight path around the fire perimeter, the return path can be determined as a clockwise rotation from the current aircraft 
location to the launch origin. As shown below, the order of rotation can be determined using a cross product relationship which checks the location of a third node to a line segment defined by the first two points. Since the convex hull connects the points in the order as they appear along the perimeter to form a convex polygon, extracting any sequential three points is sufficient to determine the rotation. The value output by this algorithm should always be negative, assuming that the return route is constructed from the launch origin to the current aircraft location.

$$
\begin{array}{cl}
\mathrm{x}_{1} & \text { Coordinate } 1 \text { distance east from origin (feet) } \\
\mathrm{y}_{1} & \text { Coordinate } 1 \text { distance north from origin (feet) } \\
\mathrm{x}_{2} & \text { Coordinate } 2 \text { distance east from origin (feet) } \\
\mathrm{y}_{2} & \text { Coordinate } 2 \text { distance north from origin (feet) } \\
\mathrm{x}_{3} & \text { Coordinate } 3 \text { distance east from origin (feet) } \\
\mathrm{y}_{3} & \text { Coordinate } 3 \text { distance north from origin (feet) } \\
\lambda=\left(\mathrm{y}_{3}-\mathrm{y}_{1}\right) \cdot\left(\mathrm{x}_{2}-\mathrm{x}_{1}\right)-\left(\mathrm{x}_{3}-\mathrm{x}_{1}\right) \cdot\left(\mathrm{y}_{2}-\mathrm{y}_{1}\right)
\end{array}
$$

If $\lambda<0$ : Rotation of points from 1, 2, to 3 is Counterclockwise If $\lambda>0$ : Rotation of points from 1,2, to 3 is Clockwise If $\lambda=0$ : Points 1,2 , and 3 are collinear

Once the rotation is determined, the next step is identifying the starting node in the solution polygon. Since a convex hull merely returns the enclosing points in a set, there is no guarantee that the algorithm has established the origin as the first point in the series. Assuming that each point added to the algorithm is listed in sequential order, the lowest node number becomes the starting node, while the last point with the highest node index will be the most recently added node. Using this information, the lowest index is sought for since there is no assurance that the origin was not excluded from the solution due to enclosure. Once this point is located, the convex hull solution is split and reorganized to ensure the solution starts at the first node and builds up to the current aircraft location. 
The actual return path construction can then begin using the newly ordered and verified solution sequence. The first step in the process is to check the identity of the first node indexed in the convex hull sequence. If the first node listed is not the origin, the coordinates for the origin are forcefully added to the return path to ensure it is the final destination. Each node indexed in the solution is then added in increasing order to the return path until the highest numbered node in the path is reached. The current aircraft location is then added as the last point in the return path. If a return solution home is being constructed then this process is repeated each time interval; however, if the return path is being followed to the home location, the same algorithm is reused, but the current aircraft location is not kept in the path. 


\section{SIMULATION EVALUTION}

The approach presented offers a practical implementation for addressing multiple UAVs within parent-child architectures and operating with the common goal of wildfire disaster and response. All the necessary components to build and demonstrate this preliminary implementation are addressed including the required architecture, communications, and algorithms. For validation, the need arises to provide a proof of concept by demonstrating the proposed concepts in simulation. Over the course of this research, three simulations were developed to prototype several key abilities: the middleware concept for communications, the HALE algorithms for search path construction and the LASE algorithms for following fire perimeters.

\subsection{Simulation Response}

Some basic assumptions were made to eliminate unnecessary complexity and focus on the core principals, the aircraft would essentially operate in two-dimensional space, the control system would allow perfect control of the aircraft, and the sensors would provide a consistently reliable image under any condition. Using these basic guidelines, three simulations were produced. The first was a series of iterations done under the initial HALE concept and served as a proving ground for the path creation algorithms. This simulation also provided the initial research and implementation with middleware communication. The second simulation created was the HALE algorithm interactive simulation created in Matlab. Using an interface, this small program illustrates how a user can define a series of search locations and have the HALE UAV algorithms determine the order to visit these locations and the manner in which to search them. The LASE 
interactive simulation demonstrates the proposed mode of handling, perimeter following, and optimal solution return algorithms.

\subsubsection{Communications Simulation and Prototype}

The simulation presented below, in Figure 39, was created as a demonstration to supplement the Autonomous Robust Avionics (AuRA) Project lead by the Intelligent Mission Management group at NASA-Ames Research Center. The intent of which was to drive toward full autonomy with UAVs under the Wildfire Research and Applications Partnership (WRAP). Much of the framework for how the HALE and LASE UAVs would play a role in autonomously collecting data and relaying this information to the USFS was researched. The HALE concept of operations was prototyped using an adaptive planner for path optimization, a graphical user interface, a sensor model, a flight control system, a middleware communication engine, and a visualization program, as shown in Table 7. From these components, the basic premise of the simulation selecting locations which were desired to be investigated for potential wildfires. Based on priority, these locations were assigned number by the user which directly correlated to the number of rings that would be created in the search pattern. When the user was finished assigning target search locations, the adaptive planner APEX would optimize the route using the 2-Opt algorithm and then load this solution into FLTz_waypoint for the aircraft flight controls to follow. 
Table 7: HALE Simulation Code Module Descriptions

\begin{tabular}{|c|l|}
\hline $\begin{array}{c}\text { Component } \\
\text { Name }\end{array}$ & \multicolumn{1}{c|}{ Simulation Module Description } \\
\hline APEX & $\begin{array}{l}\text { Produced by Dr. Michael Freed's team at NASA Ames Research Center, this module } \\
\text { provides the adaptive planning capabilities and organizes the user submitted } \\
\text { waypoints utilizing the 2-Opt algorithm. }\end{array}$ \\
\hline Moving Map & $\begin{array}{l}\text { User interface that allows creation of search nodes for the aircraft through mouse } \\
\text { clicks. This map also displays UAV current route and tracks aircraft progress. }\end{array}$ \\
\hline DOMS & $\begin{array}{l}\text { The Distributed Open Messaging System is a NASA-Ames internally created } \\
\text { publish/subscribe Ethernet based middleware. This acts as the communication link } \\
\text { between all the code modules. }\end{array}$ \\
\hline AFM & $\begin{array}{l}\text { Aircraft Fire Manager provides the sensor model and tests the aircraft line scanner } \\
\text { against circles representing randomly generated fires near the search locations. }\end{array}$ \\
\hline FLTz_Waypoint & $\begin{array}{l}\text { HALE UAV aircraft dynamics and flight control laws. This module allows the aircraft to } \\
\text { generate and follow waypoints using internal autopilot and a non-linear aircraft } \\
\text { simulation. This is coupled with DOMS_Comm to allow DOMS to write to its shared } \\
\text { memory. }\end{array}$ \\
\hline Riptide & $\begin{array}{l}\text { Graphical simulation framework produced by the U.S Army Aeroflightdynamics } \\
\text { Directorate and U.C. Santa Cruz at Ames Research Center. Allows visualization of } \\
\text { aircraft, terrain, sensor swath, and generated wildfire. This is coupled with Staterip to } \\
\text { allow DOMS to write to its shared memory. }\end{array}$ \\
\hline
\end{tabular}

In Figure 39, the communication flow is visualized for the middleware communications provided by DOMS where a publish and subscribe model was used to relay data between each of the applications since this middleware choice allows for event driven data transmission between multiple applications using a central daemon. Each of the communication links creates a topic that consists of the data type or structure being passed, the application providing data, and the name of the variable. The direction of the arrow from the square application boxes designates either a sender or receiver of the particular data. All messages being passed must reside, or pass through, the central DOMS daemon as it stores the values in local memory and notifies the appropriate applications new data is available. 


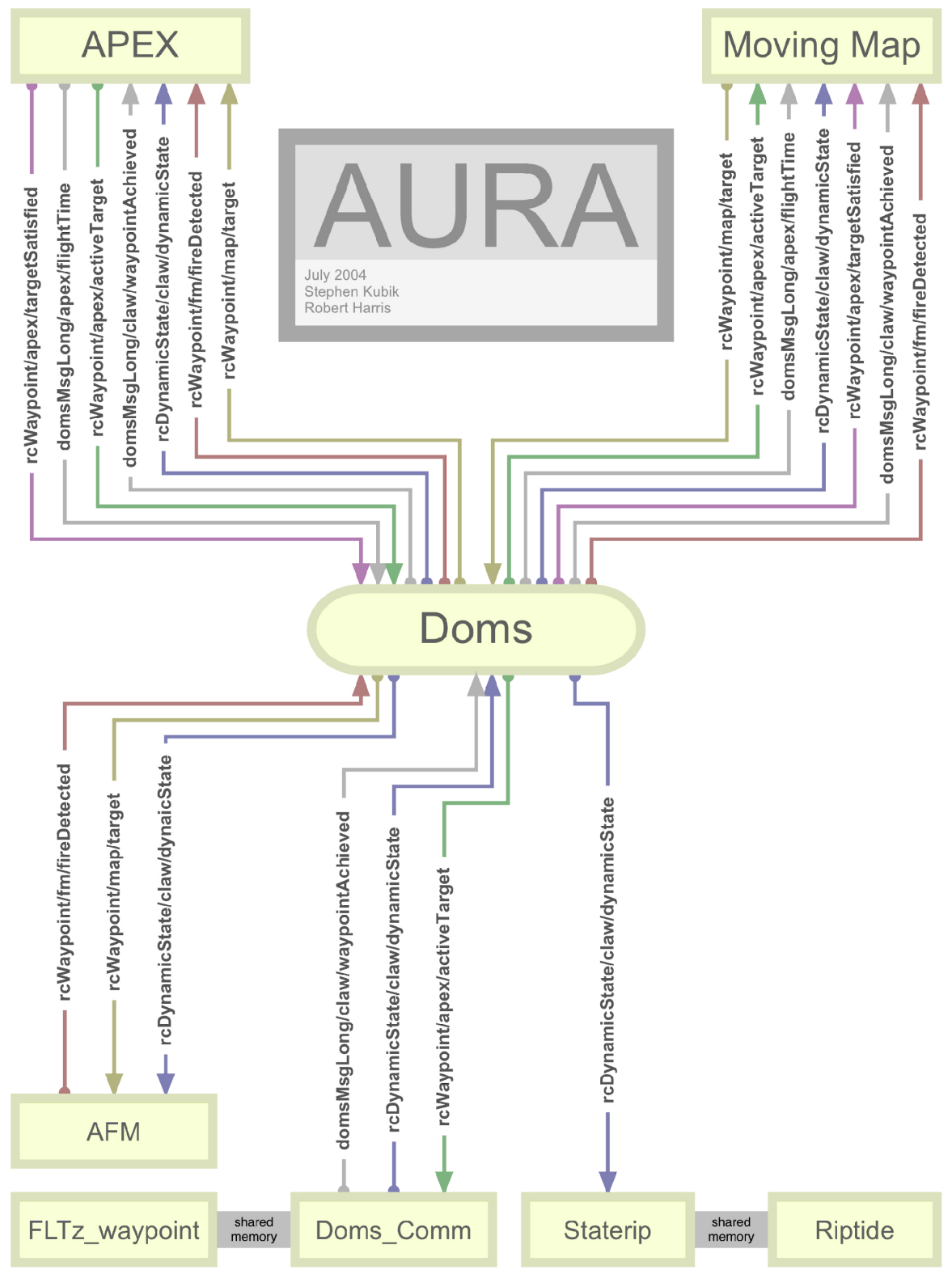

Figure 39: DOMS Middleware Architecture for the AuRA Simulation

As depicted below in Figure 40, the aircraft progress is continuously displayed on the Moving Map, FLTz_Waypoint flight plan display and Riptide graphics engine. The Moving Map in the lower right corner shows that the aircraft is approaching the white icon of a user defined search location. In the lower left, a spiral search pattern with three concentric rings has been defined in FLTz_Waypoint interface based on the user priority. The main Riptide display of the HALE UAV shows an existing wildfire in the search region. The aircraft will proceed as directed to 
follow the waypoints in the flight interface until a wildfire is detected by the sensor model AFM which is performed by a series of line-circle intersections between the fire's circular perimeter and the along track line scanner. If an intersection is found with the aircrafts line sensor, a fire signature is reported.

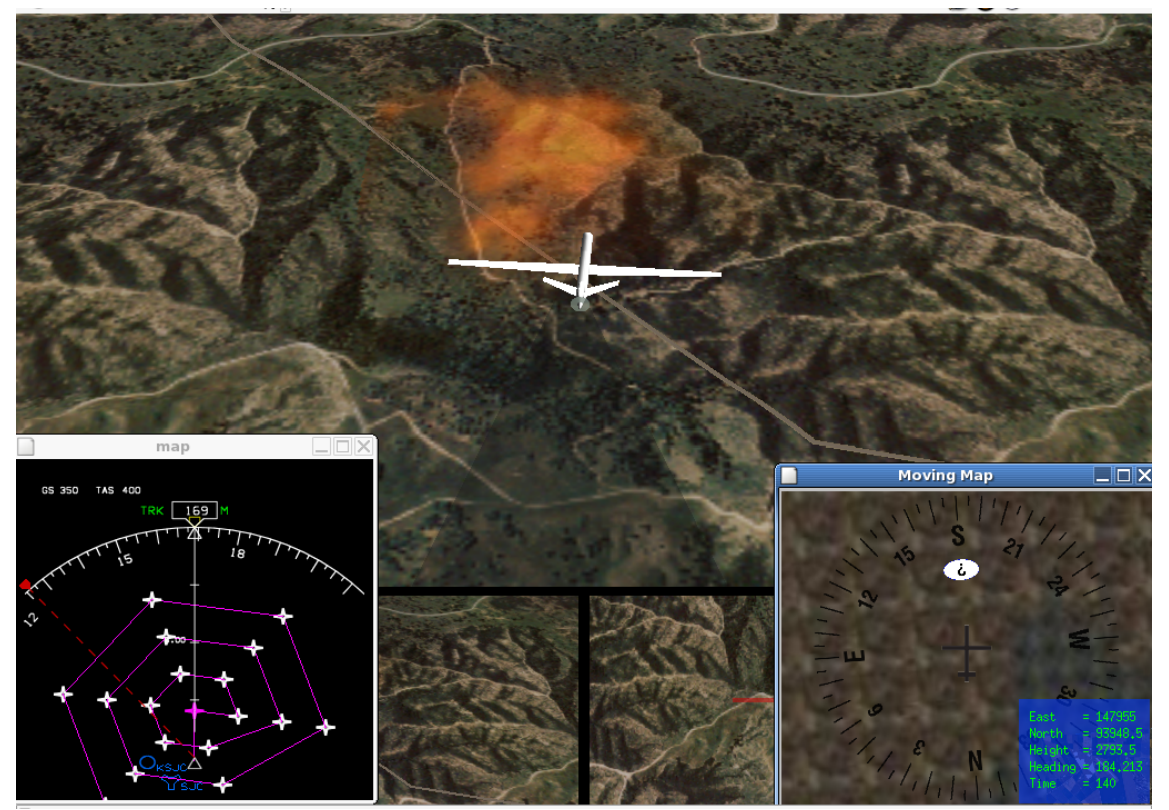

Figure 40: Aircraft Progressing Toward Search Area with a Wildfire

Once a fire is detected, as shown in Figure 41, the event is reported back to the moving map and adaptive planner which then removes the search icon and places a fire icon at the current location. The adaptive planner APEX takes the information, removes the current search location from the queue, and then re-plans the route with the 2-Opt algorithm. As noted in the FLTz_Waypoint interface, the aircraft has already turned to track a new trajectory toward the next search location nearby. The flight control interface is also presented on the bottom of this diagram, but no functions are toggled by the user since the aircraft is capable of full autonomy with all the loop closures operating. 


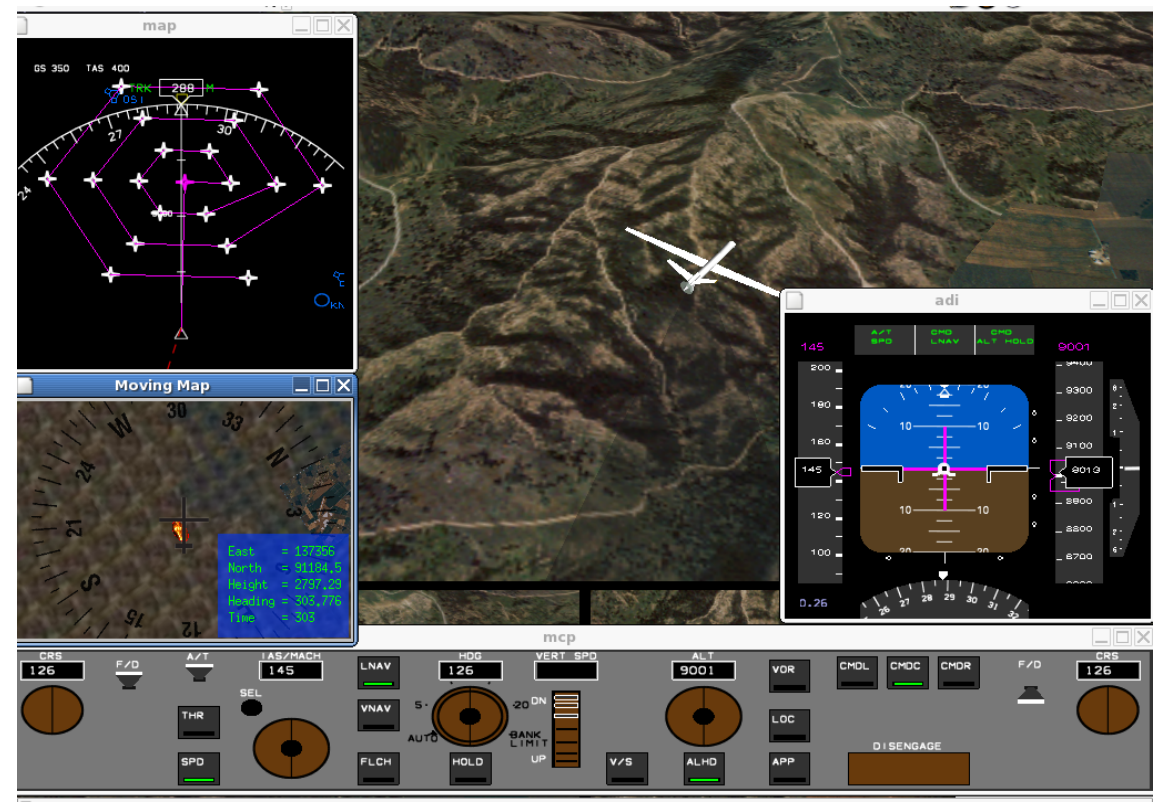

Figure 41: Aircraft Locating Wildfire and Proceeding to Next Target

While this simulation was successful in demonstrating the HALE algorithms with a non-linear aircraft in a time based environment and with interactions with various software components several shortcomings were noted in the implementation. The designation of search rings based on user priority proved a drawback since the area spent searching for a wildfire was on an algorithmic decision process. In reality, it would be more likely that a search region would be defined with a user defined priority which avoids having to create multiple searches in an effort to encompass a desired search region and bypass the priority allocation method. The algorithm naturally assumed a single fire existed for each search location. The potential for two wildfires to exist in the same search area is likely in the real world situations, and with this methodology; the aircraft would find one, then move to the next target. This provides even more rationale to move to a fixed search area as the vehicle will be forced to search all of the user defined area. The spiral search pattern is potentially problematic as the sensor swath may be skewed based on the bank angle of the aircraft which ultimately distorts the detected image making it harder for the vehicle to consistently overlap with its last track. A linear switchback pattern would then allow 
for easier overlapping of sensor swaths, preventing the vehicle from collecting IR data while flying into, or away from, the sun by setting the appropriate heading angle. Whenever a fire is detected, or a new point is added, the adaptive planner would re-plan the route which made minor additions to the route unpredictable because the re-planned route would start from scratch and usually find a new solution. Later simulations would need to address this by ensuring that points were incorporated into the route, in a cost efficient manner, rather than re-planning the entire set.

\subsubsection{HALE Interactive Simulation}

Using the information collected in the previous simulation study, a few modifications were made concerning the HALE algorithm implementation. The first of these changes was to ensure the search area was being handled using a switchback manner as opposed to a spiral pattern. The user then defined a required search area, directly instead of a priority value, that defines search area. Using these principles, the following Matlab code was constructed to demonstrate the HALE UAV algorithms, contained in Appendix H. As noted in Figure 42, the HALE launch location has already been set, and the user selected a series of outlining coordinates that defined a search region. Once the users' selection is complete, another click created a blue polygon designating a desired search region. The user can continue defining search area polygons in this

fashion until all desired search regions are created, and then passes control over to the HALE algorithms. 

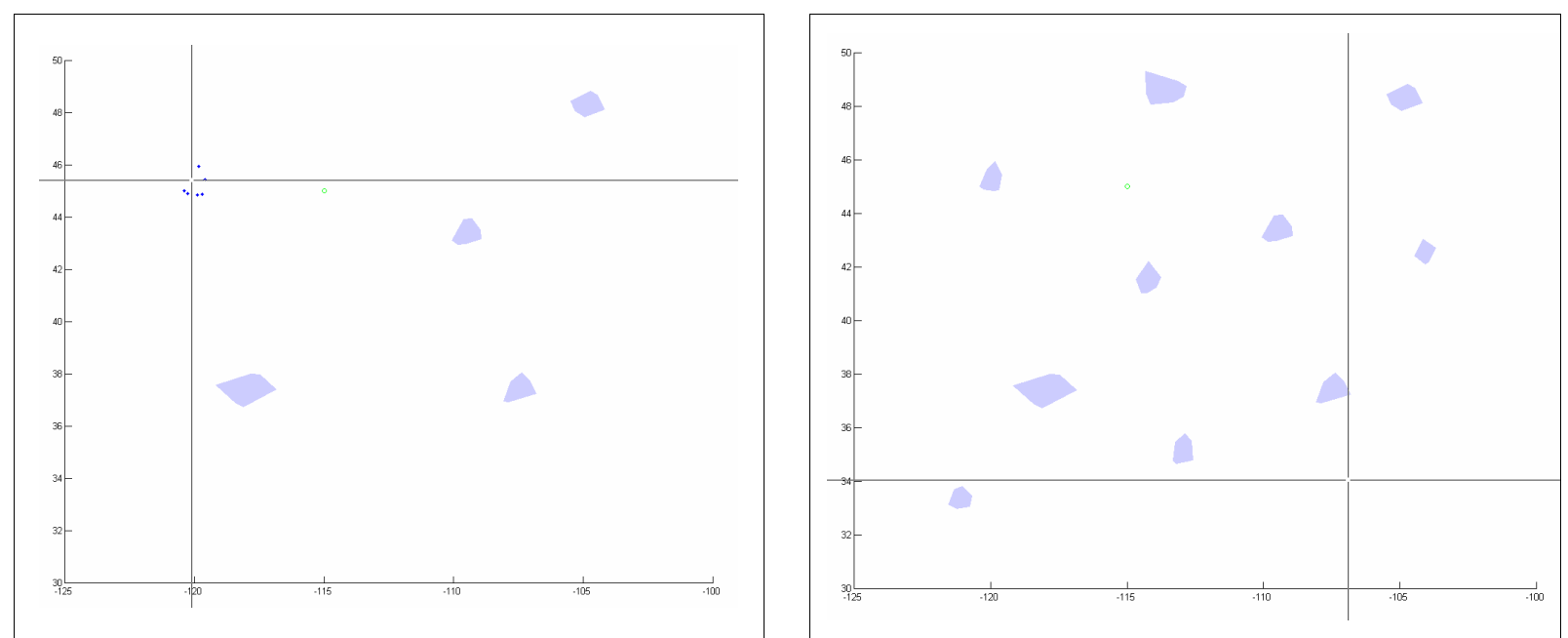

Figure 42: User Selection of HALE UAV Coordinates for Search Regions

Once the user has completed selecting search regions the approximate centroid of each of the polygons is located, as shown in Figure 43 below. These locations are computed by taking an average of each of the coordinates, depicted using a red dot in the center of each blue polygon, and are then run through the greedy and the 2-Opt algorithms to create an initial tour and then optimize it. The result is plotted using a dashed green line between each of the red dots of the search areas. The optimal path creates a complete circuit through each search region and the HALE origin. 


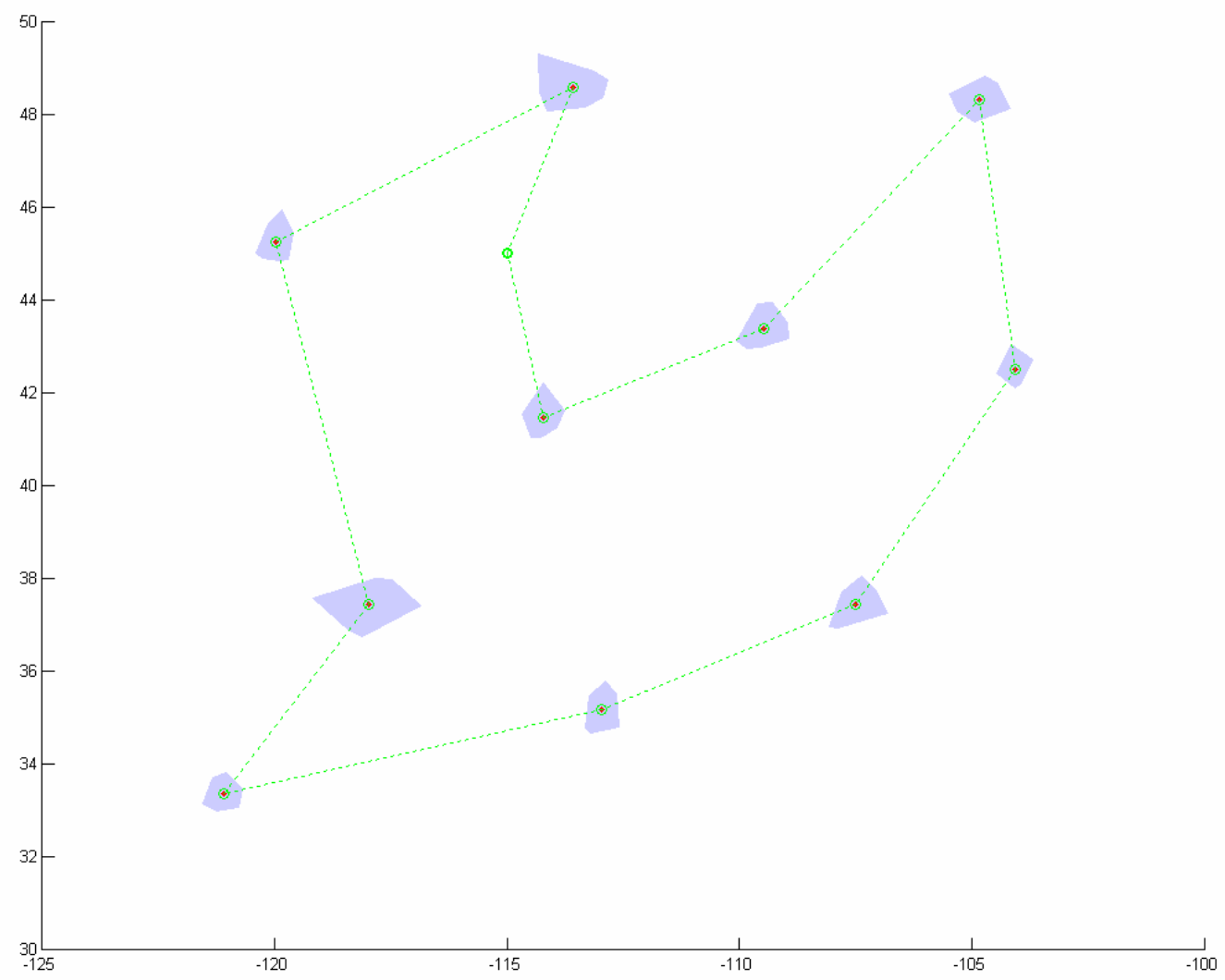

Figure 43: HALE UAV with Optimized Route through Each Search Region

As illustrated in Figure 44, the initial optimized search route is used as a basis to determine the search pattern required for each region. Using this tour of the search regions, along with the bearing of the vehicle to the next search location, are fed into the linear search algorithm to create a switchback search pattern across the entire polygon. Then, these switchback search patterns appended together with the route sequence. As a result, the optimized tour through all the target regions, as depicted with the dashed green line. The dark blue line mirrors the search pattern presented in the HALE search algorithms section for each search area. 


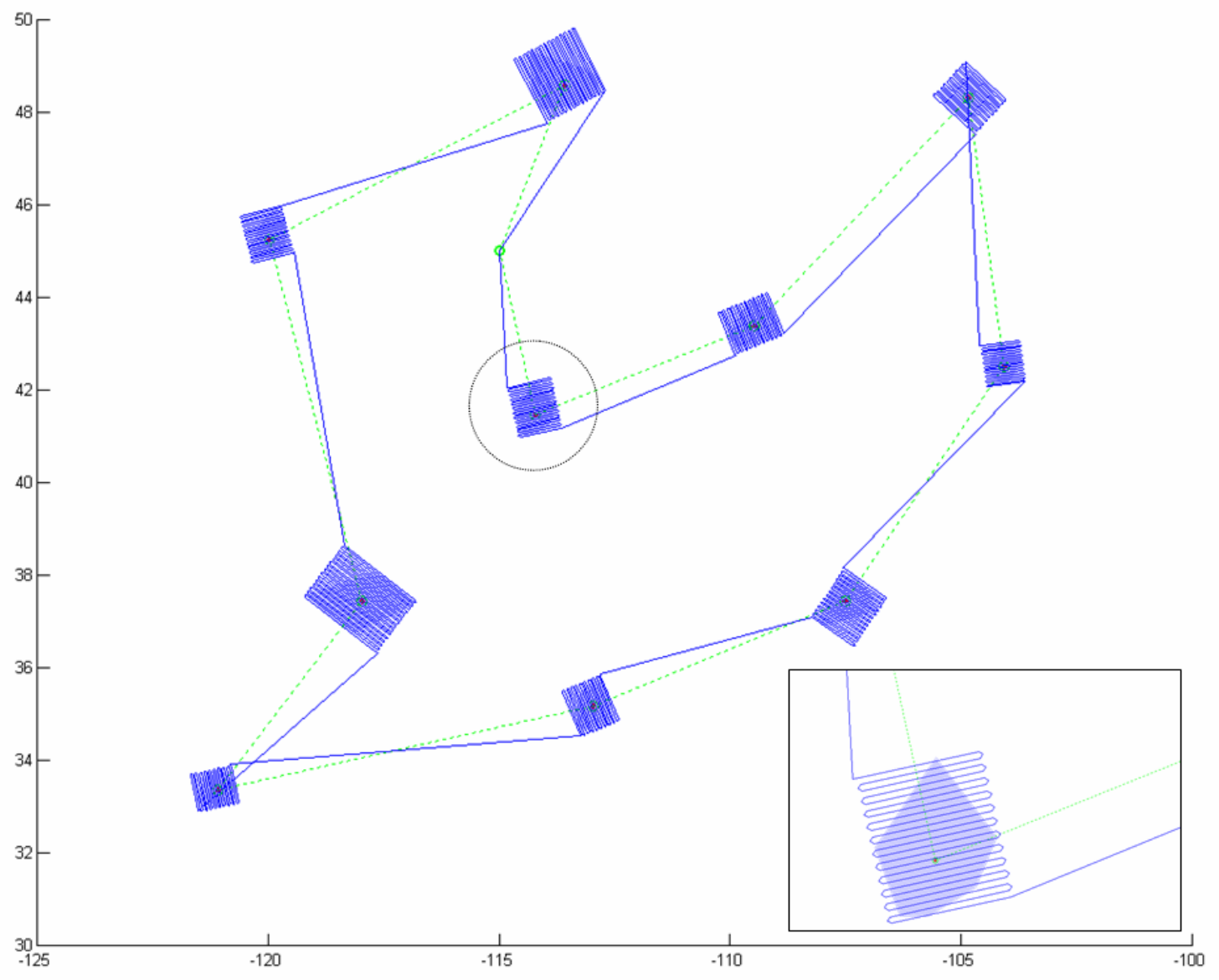

Figure 44: HALE UAV with Linear Search Pattern Based on Optimal Routing

The HALE UAV algorithm has several limitations. A byproduct of not having a time based, nonlinear aircraft model flying the full route, which includes handling when a new point is added to an existing route, can be accommodated utilizing the insert cheapest algorithm to add the point in the most cost efficient manner. Additionally, no contingency has been added to deal with the length of the tour or fuel constraints on the vehicle which allows for the assumption that the vehicle will take a consistent fuel estimate and return to the launch location when it is no longer feasible to carry on. Also, the cost matrix must incorporate more parameters aside from distance with the algorithms verified against a non-linear approach. Investigation may also be required to 
determine what sorts of properties should be included when creating the cost matrix such as area flammability, severe weather avoidance, potential threat of fire, and vehicle limitations.

\subsubsection{LASE Interactive Simulation}

The interactive LASE simulation was constructed in Matlab as validation for the approach presented in Section 3.4. As part of this simulation, the user is capable of selecting only two locations in a displayed map. The first location selected is the aircraft launch coordinates, marked by a green circle, and defines where the LASE UAV will both start from and end at when a low fuel flag is registered. The second coordinate, marked by a red circle, is equivalent to the HALE UAV detected fire signature that has been transmitted to the local ground station. Once this coordinate is assigned, the user simulates the reported location of an over passing HALE UAV and launches the LASE UAV to investigate. This interface holds the outline of a red colored fire region that is not yet 'known' to the user or the LASE UAV which represents a wildfire occurring in the region and allows the user to experiment with different launch locations, different destination locations, changing fire perimeter geometry, and potentially investigate if the LASE UAV is capable of handling the interactions.

As shown in Figure 45, the left image shows the selection process for marking the suggested fire signature location after the user has already chosen a LASE launch location. The image on the right demonstrates the execution of a LASE UAV simulation, the trail of blue points representing the aircraft locations. At the end of the trail, designating the current UAV location, a green line represents the instantaneous optimal return path calculated for the LASE which allows for calculation of fuel usage estimation on the return trip home. When this estimation is combined 
with how much fuel has already been used in the distance traveled, the aircraft can assess when the optimal return path must be followed back to the UAV launch location.
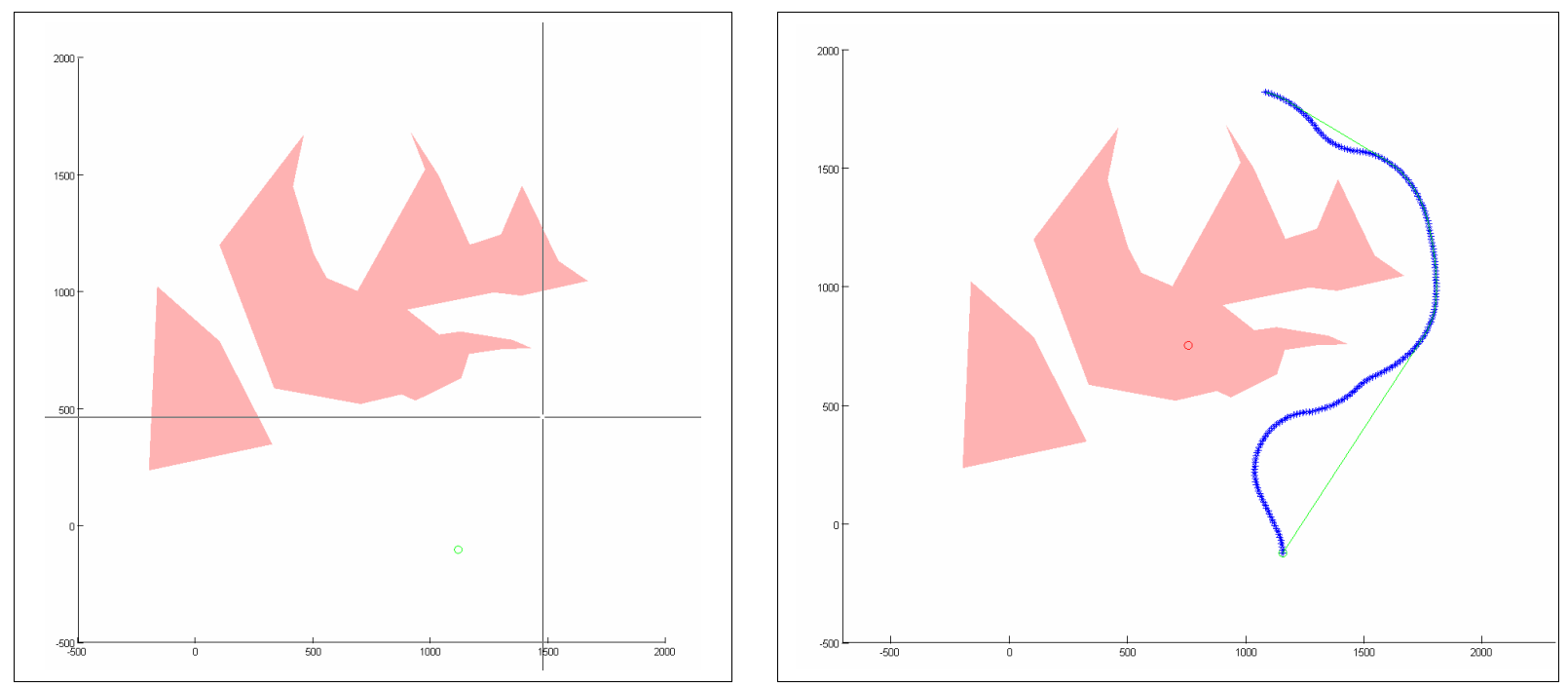

Figure 45: Selection of LASE Coordinates and Aircraft Tracking Response

Figure 46, below, exhibits how the aircraft leaves the launch location at a specific heading and then corrects the trajectory until the aircraft is headed for the potential wildfire signature. While in route, the aircraft detects a fire perimeter and begins tracking the wildfire using the angle correction method. At each time interval, the aircraft computes a new return path back to the launch location and adds the current distance traveled to determine if sufficient fuel is available. When the aircraft estimates it must return home, the LASE UAV will turn to the right and then begin following an optimal return path that shortens the overall distance back. Upon its return, the simulation is stopped where the UAV would land and ready itself for redeployment. 


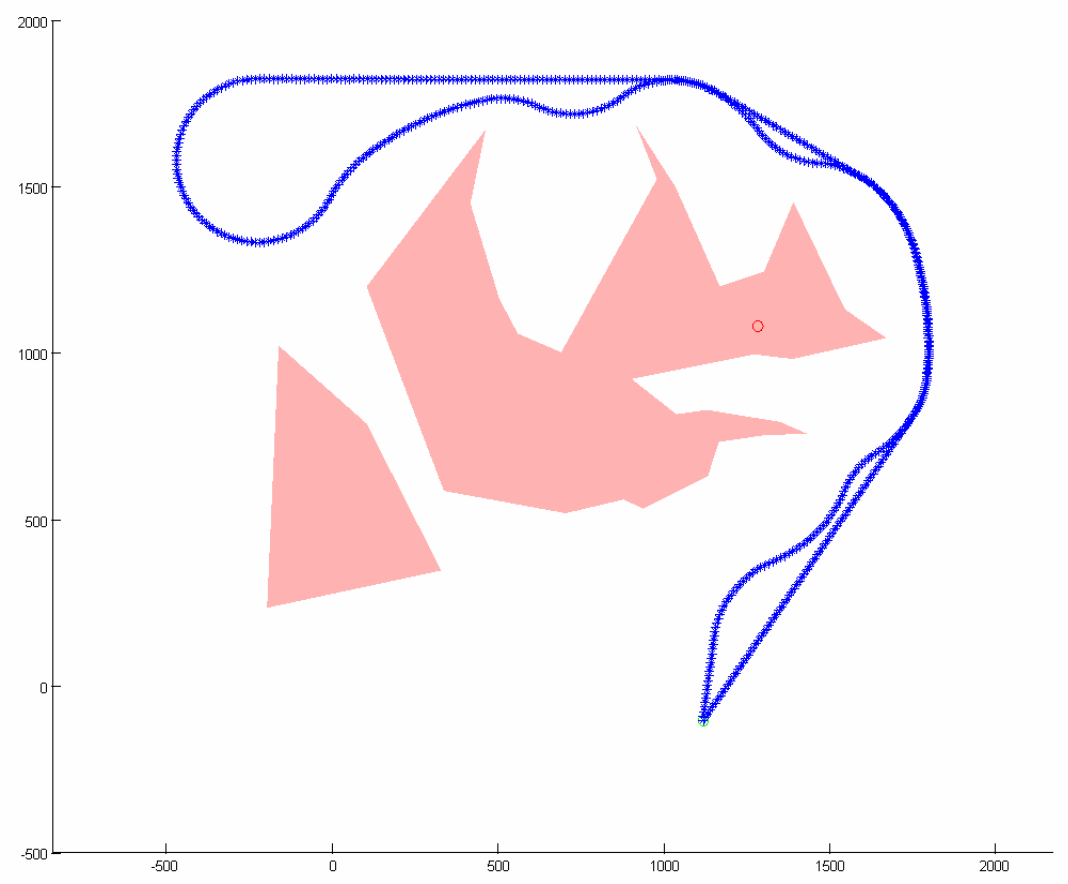

Figure 46: Completed LASE Simulation with Fire Perimeter Tracking

The alternative mission to Figure 46 takes place when an invalid fire signature is sent from the HALE UAV and, in response, the LASE UAV is sent out to investigate. This sequence is initiated when the user selects a launch location and potential fire signature far away from the real wildfire perimeter. As shown below in Figure 47, the aircraft is launched from the initial location selected and then adjusts trajectory to head for the representative HALE transmitted wildfire signature chosen by the user. The aircraft proceeds, constantly checking if a wildfire perimeter is in the field of view of the vehicle and using the distance traveled as the amount of distance to return which continues until the aircraft arrives at the coordinate and determines that no fire has been detected. The aircraft will then proceed back to the launch point and the simulation will conclude. 


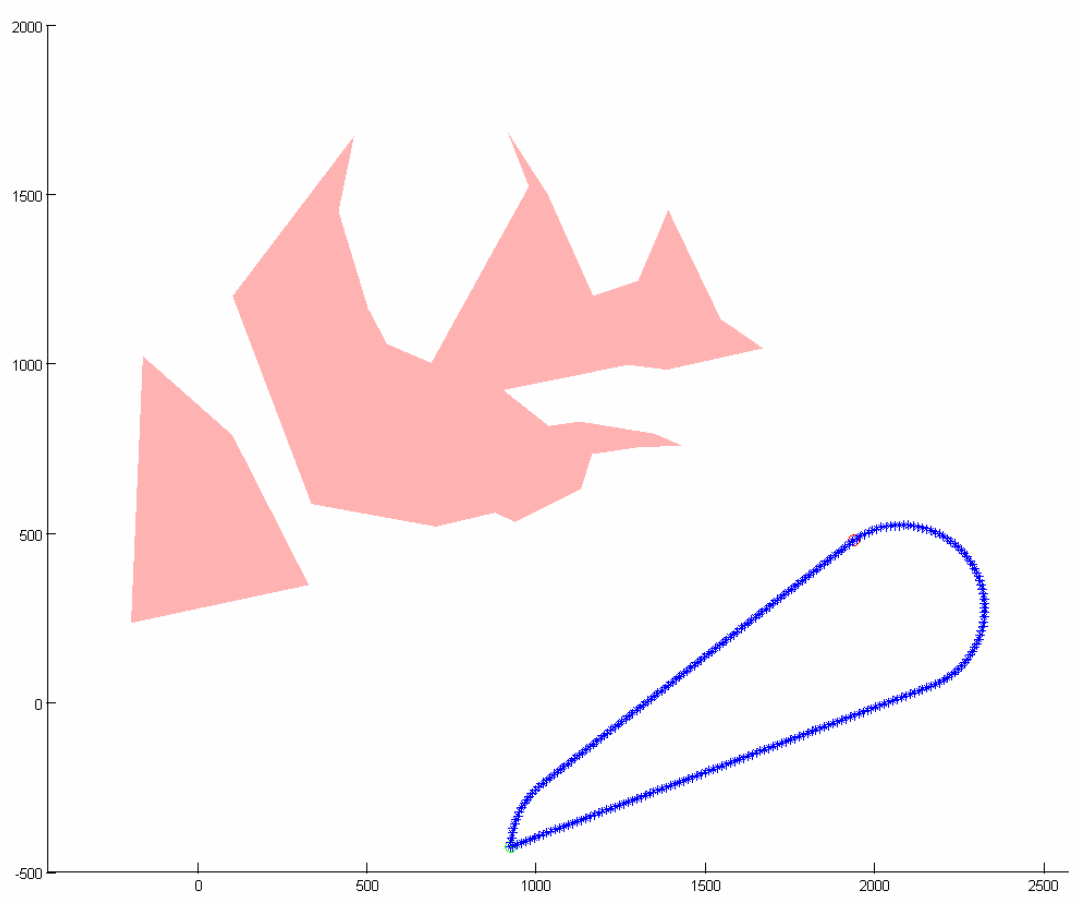

Figure 47: Completed LASE Simulation with No Wildfire Located

While the simulation has proven the algorithm design is capable to handle the majority of the conditions presented, several key areas are still lacking development. Checks for the algorithm currently do not exist when the vehicle has finished a complete circuit around the fire perimeter which was left out to reduce complexity in the algorithm and not force a design decision as to how the vehicle reacts when completing a single loop. Non-linear behavior does not exist in the simulation including gust handling, differences in airspeed versus groundspeed, and vehicle dynamics which was due to the lack of availability of a LASE helicopter model with a flight control system and flight director. As a result, the aircraft was assumed to perfectly follow all commanded behavior and achieve the required bank angle for turns instantly. The simulation also does not tackle the concept of a fire perimeter that is capable of changing in time which may have massive repercussions on fuel ranging and require the constant use of the sensor model for the return path home. Presently the simulation assumes a static perimeter. Lastly, the sensor model assumes the vehicle is never changing altitude and the ground is not changing elevation 
which is necessary to be incorporated for a realistic simulation as it may procure consequences on sensor field of view, as examined previously.

\section{CONCLUSIONS}

The algorithms provided here have established practicality for a fully autonomous wildfire response mission; the ultimate goal has been to demonstrate that autonomous cooperation is possible for missions where both active and passive response is required. By separating the mission into simple objectives, LASE and HALE UAVs are exceptionally capable of completing these tasks with little to no human interaction. The only critical aspect becomes ensuring that the data is passed between these autonomous assets so that each UAV can carry out the task in an efficient manner.

Additionally, the mission proposed here heavily pushes toward introduction of unmanned aircraft into the commercial sector. While obstacles such as access to national airspace and available UAV technology must be overcome before this implementation can become a reality, the architecture does not necessarily hinge on UAVs. The civil sector should note that the architecture is capable of scaling between any set of assets that are capable of carrying out autonomous tasks. Just as one aircraft can command another, the concept can be implemented between satellites that are passing route information to an aircraft flying border security missions. As stated, just as autonomous is not constrained to military applications; the research presented should serve as a viable template for any application in the civil sector. 


\section{REFERENCES}

1 T. Cox, C. Nagy, M. Skoog, and I. Somers (2004) Civil UAV Capability Assessment, Prepared for NASA Aeronautics Research Mission and Science Mission Directorates

2 Office of the Secretary of Defense (2007) Unmanned Aircraft Systems Roadmap 2007-2032, Report to the Office of the Secretary of Defense

3 Office of the Secretary of Defense (2005) Unmanned Aircraft Systems Roadmap 2005-2030, Report to the Office of the Secretary of Defense

4 M. Cummings (2004) Human Supervisory Control of Swarming Networks

5 T. Leaute and B. Williams (2005) Coordinating Agile Systems Through The Model-Based Execution of Temporal Plans, Workshop on Multi-agent Planning and Scheduling 2005

6 T. Rauenbusch, S. Shieber, and B. Grosz (2005) Computing the Communications Costs of Item Allocation, Workshop on Multi-agent Planning and Scheduling 2005

7 M. Freed, R. Harris, and M. Shafto (2004) Human-Interaction Challenges in UAV-Based Autonomous Surveillance

8 A. Wehowsky, S. Block, and B. Williams (2005) Robust Distributed Coordination of Heterogeneous Robots through Temporal Plan Networks, Workshop on Multi-agent Planning and Scheduling 2005

9 D. Han and S. Barber (2005) Determining Task Valuations for Task Allocation, Workshop on Multiagent Planning and Scheduling 2005

10 UAVs for Land Management \& Coastal Zone Dynamics Workshop (2005) Conference Proceedings July $26^{\text {th }}-27^{\text {th }}$ Monterey, California

11 Civil UAV Assessment Team (2005) Civil UAV Capabilities Assessment, Interim Status Report

S. MacSween-George (2003) A Public Opinion Survey - Unmanned Aerial Vehicles For Cargo,

12 Commercial, and Passenger Transportation, $2^{\text {nd }}$ AIAA "Unmanned Unlimited" Systems, Technologies, Operations

13 M. DeGarmo and G. Nelson (2004) Prospective Unmanned Aerial Vehicle Operations in the Future National Airspace System

14 J. Dunion, J. Cione, R. Rogers, P. Dodge, J. Kaplan, S. Aberson, S. Murillo, and N Dorst (2006) 2006 Hurricane Field Program Plan

15 Aircraft Owners and Pilots Association (2006) Newsletter January $9^{\text {th }}, 2006$ http://www.aopa.org/whatsnew/newsitems/2006/060109uav.html

16 S. Jensen (2005) Ikhana Technical Review, October $7^{\text {th }} 2005$ 
18 V. Ambrosia (2003) UAVs for Disaster Management

19 J. Totah, C. Frost, F. Enomoto (2004) Vehicle Systems Program - Intelligent Mission Management

20 NASA-Ames Research Center (2005) AuRA Annual Report

Milebrook Technology Inc. (2000) Applications of High-Altitude Long Endurance (HALE)

21 Platforms in Emergency Preparedness and Disaster Management and Mitigation, Report for Office of Critical Infrastructure and Emergency Preparedness

22 K. Wong, and C. Bil (2006) UAVs Over Australia - Market and Capabilities

23 V. Ambrosia (2003) Disaster Management Applications - Fire, Presentation for $30^{\text {th }}$ International Symposium on Remote Sensing of Environment

24 NASA, FAA, DOD, UNITE (2003) Access Five Project - Partnership to Achieve Routine NAS Access for HALW ROA, UAV 2003 Paris Conference Proceedings June 11, 2003

25 J.R Wilson (2005) UAV Worldwide Roundup, Aerospace America September 2005 Volume 43 Number 9

26 V. Ambrosia, J. Brass, R. Higgins (2002) AIRDAS - Airborne Infrared Disaster Assessment System Brochure

27 T. Lillesand, R. Kiefer, and J. Chipman, (2004) Remote Sensing and Image Interpretation, Fifth Ed. 


\section{APPENDIX A: Worldwide UAV Data}

* Designates outliner data and removed from trend analysis.

Table 8: Worldwide Existing UAV Listing 2005

\begin{tabular}{|c|c|c|c|c|c|c|c|c|}
\hline Country & Designation & Propulsion & $\begin{array}{l}\text { Gross } \\
\text { (lb.) }\end{array}$ & $\begin{array}{l}\text { Payload } \\
\text { (lb.) }\end{array}$ & $\begin{array}{c}\text { Wingspan } \\
\text { (ft.) }\end{array}$ & $\begin{array}{c}\text { Endurance } \\
\text { (h.) }\end{array}$ & $\begin{array}{l}\text { Range } \\
\text { (n. mi.) }\end{array}$ & $\begin{array}{l}\text { Ceiling } \\
\text { (ft.) }\end{array}$ \\
\hline Australia & Vindicator & Piston & 1050 & 200 & 23.1 & 20 & 110 & 25000 \\
\hline Australia & Mark 3 & $\begin{array}{l}\text { Electronic Fuel- } \\
\text { Injected }\end{array}$ & 33 & 8.8 & 9.4 & 5 & 300 & 20000 \\
\hline Austria & Eyrie Mk 7 & Piston & 496 & 165 & 16.5 & 15 & 32 & 15000 \\
\hline Bulgaria & Yastreb-25 & Piston & 138 & 10 & 11.5 & 4 & 27 & 3000 \\
\hline Canada & CL-289 & Turbojet & 650 & 75 & 4.3 & 0.5 & 108 & 3900 \\
\hline Canada & CL-89 & Turbojet & 238 & 40 & 3.1 & 0.2 & 32 & 10000 \\
\hline China & ASN-206 & Piston & 489 & 110 & 19.7 & 8 & 81 & 19500 \\
\hline China $^{*}$ & Chang Hong 1 & Turbojet & 3750 & 140 & 32 & 3 & 1500 & 57400 \\
\hline China & DP-4 RD & Piston & 309 & 66 & 14.1 & 2 & 54 & 9800 \\
\hline China & Observer 1 & Piston & 22 & 5 & 8.8 & 1.2 & 3 & 5000 \\
\hline Croatia & BL-50 & Piston & 117 & 46 & 13.1 & 5 & 45 & 5000 \\
\hline Croatia & BLS B & Piston & 84 & 32 & 13.1 & 3 & 27 & 5000 \\
\hline Crzech Republic & Sojka III & Piston & 320 & 66 & 13.4 & 2 & 54 & 6600 \\
\hline Finland & Ranger & Piston & 595 & 86 & 18.8 & 5 & 54 & 14700 \\
\hline France & Azimut & Battery & 14 & 1 & 11.3 & 2.5 & 6 & 1000 \\
\hline France & Brevel & Piston & 330 & 66 & 11.2 & 6 & 108 & 9800 \\
\hline France & Chacal 2 & Piston & 170 & 40 & 8.7 & 4 & 27 & 10000 \\
\hline France & Crecerelle & Piston & 298 & 77 & 10.8 & 5 & 49 & 13100 \\
\hline France & DRAC & Battery & 16.5 & 3.9 & 11.7 & 1.5 & 6 & 6500 \\
\hline France & Dragon & Rotary & 331 & 66 & 9.8 & 2 & 27 & 9600 \\
\hline France & Fox AT2 & Piston & 265 & 55 & 11.8 & 5 & 81 & 11500 \\
\hline France & Fox TX & Piston & 265 & 66 & 11.8 & 5 & 81 & 11500 \\
\hline France & Hussard 2 & Piston & 66 & 18 & 11.5 & 1 & 4 & 6600 \\
\hline France & K-100 & Piston & 62 & 11 & 8.5 & 0.5 & 8 & 9600 \\
\hline France & MART Mk II & Piston & 242 & 55 & 11.2 & 4 & 54 & 9800 \\
\hline France & Marula & Rotary & 298 & 77 & 7.2 & 5 & 54 & 13100 \\
\hline France & MK-106C Hit & Piston & 220 & 50 & 10.5 & 3 & 27 & 12000 \\
\hline France & Scorpio & Piston & 1500 & 397 & 23.5 & 8 & 80 & 12000 \\
\hline France & S-Mart & Piston & 318 & 66 & 11.2 & 7 & 81 & 9800 \\
\hline France & Sperwer/Ugglan & Piston & 573 & 99 & 13.8 & 8 & 81 & 16400 \\
\hline France & Taifan & Piston & 330 & 50 & 11.8 & 4 & 54 & 12000 \\
\hline Germany & Predator (RQ-1A) & $\begin{array}{l}\text { 4-Cylinder } \\
\text { Piston Rotax }\end{array}$ & 2250 & 450 & 48.7 & 40 & 400 & 25000 \\
\hline India & Heron & Piston & 2400 & 550 & 54.5 & 50 & 125 & 30000 \\
\hline India & Nishant & Rotary & 771 & 132 & 21.3 & 4 & 54 & 13100 \\
\hline India & Searcher & Piston & 820 & 139 & 23.7 & 14 & 65 & 15000 \\
\hline Iraq & L-29 & Piston & 7200 & 1200 & 33.8 & 1 & 344 & 25000 \\
\hline Isreal & Blue Horizon & Piston & 286 & 77 & 16.4 & 16 & 50 & 20000 \\
\hline
\end{tabular}




\begin{tabular}{|c|c|c|c|c|c|c|c|c|}
\hline Country & Designation & Propulsion & $\begin{array}{l}\text { Gross } \\
\text { (lb.) }\end{array}$ & $\begin{array}{l}\text { Payload } \\
\text { (Ib.) }\end{array}$ & $\begin{array}{c}\text { Wingspan } \\
\text { (ft.) }\end{array}$ & $\begin{array}{c}\text { Endurance } \\
\text { (h.) }\end{array}$ & $\begin{array}{l}\text { Range } \\
\text { (n. mi.) }\end{array}$ & $\begin{array}{c}\text { Ceiling } \\
\text { (ft.) }\end{array}$ \\
\hline Isreal & Canard & Piston & 77 & 12 & 7.9 & 6 & 54 & 3000 \\
\hline Isreal & Colibri & Piston & 79 & 22 & 13.2 & 1 & 27 & 6600 \\
\hline Isreal & Crow & Piston & 287 & 55 & 16.8 & 9 & 108 & 15000 \\
\hline Isreal & Darter & Rotary & 220 & 22 & 14 & 4 & 50 & 20000 \\
\hline Isreal & Hermes 1500 & Piston & 3300 & 500 & 32.8 & 36 & 108 & 30000 \\
\hline Isreal & Hermes 4505 & Rotary & 992 & 220 & 34.4 & 20 & 108 & 20000 \\
\hline Isreal & Heron & Piston & 2756 & 551 & 54.5 & 40 & 108 & 30000 \\
\hline Isreal & Hunter & Piston & 1600 & 251 & 29.2 & 10 & 160 & 15000 \\
\hline Isreal & Hunter (RQ-5) & Piston & 1600 & 150 & 29.2 & 11 & 144 & 15000 \\
\hline Isreal & I-View & Piston & 364 & 66 & 18.7 & 6 & 43 & 20000 \\
\hline Isreal & Micro-V & Piston & 100 & 18 & 11.8 & 5 & 27 & 15000 \\
\hline Isreal & Mini Sheddon & Piston & 55 & 10 & 9.8 & 3 & 5 & 12000 \\
\hline Isreal & Ranger & Piston & 606 & 100 & 18.73 & 5 & 80 & 15000 \\
\hline Isreal & Scout & Piston & 350 & 84 & 16.3 & 7 & 54 & 15000 \\
\hline Isreal & Searcher & Piston & 939 & 220 & 28 & 16 & 108 & 20000 \\
\hline Isreal & Searcher Mk II & Piston & 940 & 150 & 28.1 & 16 & 80 & 18500 \\
\hline Isreal & Sheddon MK3 & Piston & 90 & 18 & 13.5 & 6 & 24 & 15000 \\
\hline Isreal & Sniper & Rotary & 375 & 55 & 13.8 & 6 & 27 & 15000 \\
\hline Isreal & Vanguard & Piston & 386 & 88 & 19.7 & 16 & 108 & 15000 \\
\hline Italy & Mirach 100 & Turbojet & 617 & 88 & 5.9 & 1 & 135 & 29500 \\
\hline Italy & Mirach 150 & Turbojet & 750 & 110 & 8.5 & 1 & 135 & 29500 \\
\hline Italy & Mirach 26 & Piston & 463 & 77 & 15.5 & 6 & 27 & 11500 \\
\hline Phillipines & Blue Horizon & Piston & 286 & 77 & 16.4 & 16 & 50 & 20000 \\
\hline Portugal & Armor X7 & Piston & 440 & 110 & 19.7 & 12 & 216 & 6600 \\
\hline Russia & Tu-143 & Turbojet & 3060 & 400 & 7.3 & 0.2 & 100 & 9800 \\
\hline Russia & Tu-243 Reys-DM & Turbojet & 3100 & 500 & 7.3 & 0.4 & 216 & 16400 \\
\hline Singapore & Searcher & Piston & 820 & 139 & 23.7 & 14 & 65 & 15000 \\
\hline South Africa & Lark & Rotary & 265 & 55 & 6.9 & 4 & 54 & 15000 \\
\hline South Africa & RPV-2E Seeker & Piston & 529 & 88 & 23 & 8 & 108 & 18000 \\
\hline South Africa & Vulture & Piston & 220 & 55 & 16.1 & 3 & 32 & 16400 \\
\hline South Korea & TRPV-1 Doyosae & Rotary & 286 & 50 & 15.8 & 3 & 27 & 6000 \\
\hline Spain & SIVA & Piston & 660 & 220 & 15.8 & 6.5 & 90 & 9800 \\
\hline Sri Lanka & Scout & Piston & 350 & 84 & 16.3 & 7 & 54 & 15000 \\
\hline Sweden & ADID 55 & Piston & 330 & 121 & 10.75 & 6 & 30 & 9750 \\
\hline Switzerland & ADS-95 Ranger & $\begin{array}{l}\text { Pusher-Prop } \\
\text { Piston }\end{array}$ & 595 & 86 & 18.8 & 5 & 54 & 14700 \\
\hline Taiwan & Kestrel II & Piston & 250 & 60 & 16.5 & 5 & 27 & 8000 \\
\hline Thailand & Searcher & Piston & 820 & 139 & 23.7 & 14 & 65 & 15000 \\
\hline \begin{tabular}{|l|} 
Tunisia \\
\end{tabular} & Annasnas & Piston & 275 & 55 & 12.5 & 14 & 54 & 16000 \\
\hline Turkey & Dogan & Piston & 364 & 40 & 19.7 & 12 & 81 & 20000 \\
\hline Turkey & Firefly & Piston & 110 & 10 & 13.8 & 5 & 27 & 12000 \\
\hline Turkey & Kirlangic & Piston & 298 & 30 & 16.8 & 8 & 81 & 20000 \\
\hline Turkey & UAV-X1 & Rotary & 540 & 66 & 19.7 & 7 & 16 & 15000 \\
\hline
\end{tabular}




\begin{tabular}{|c|c|c|c|c|c|c|c|c|}
\hline Country & Designation & Propulsion & $\begin{array}{l}\text { Gross } \\
\text { (lb.) }\end{array}$ & $\begin{array}{c}\text { Payload } \\
\text { (Ib.) }\end{array}$ & $\begin{array}{c}\text { Wingspan } \\
\text { (ft.) }\end{array}$ & $\begin{array}{c}\text { Endurance } \\
\text { (h.) }\end{array}$ & $\begin{array}{l}\text { Range } \\
\text { (n. mi.) }\end{array}$ & $\begin{array}{c}\text { Ceiling } \\
\text { (ft.) }\end{array}$ \\
\hline United Kingdom & ASR-4 Spectre & Rotary & 242 & 81 & 10.8 & 3 & 27 & 17000 \\
\hline United Kingdom & CSV-20 & Piston & 44 & 8 & 9.3 & 2 & 4 & 5000 \\
\hline United Kingdom & MSV-20 & Piston & 9 & 1 & 9.1 & 0.5 & 3 & 2000 \\
\hline United Kingdom & Phantom & Piston & 77 & 18 & 8.2 & 3 & 16 & 9800 \\
\hline United Kingdom & Phoenix & Piston & 386 & 110 & 18.1 & 5 & 32 & 8000 \\
\hline United Kingdom & Raven & Piston & 185 & 45 & 12 & 3 & 54 & 14100 \\
\hline United Kingdom & Specte II & Piston & 320 & 50 & 10.5 & 5 & 27 & 13000 \\
\hline United States & Aerosonde & $\begin{array}{l}\text { Electronic Fuel- } \\
\text { Injected }\end{array}$ & 31 & 11 & 9.6 & 50 & 1800 & 21000 \\
\hline United States & AHMMH-1 & Piston & 16 & 2 & 5 & 3 & 3 & 5000 \\
\hline United States & Altus-1 ST & Piston & 1600 & 300 & 55.3 & 30 & 250 & 45000 \\
\hline United States & Altus-2 DT & Piston & 1600 & 300 & 55.3 & 6 & 300 & 65000 \\
\hline United States* & Arrow & Turbofan & 14000 & 500 & 115.3 & 36 & 120 & 70000 \\
\hline United States & Backpack & Piston & 25 & 4 & 3 & 1 & 5 & 5000 \\
\hline United States & BAT & Piston & 15 & 4 & 5 & 6 & 180 & 9000 \\
\hline United States & BMQ-74C & Turbojet & 514 & 173 & 5.8 & 1 & 200 & 30000 \\
\hline United States & Boomerang & $\begin{array}{l}\text { 1-Cylinder } \\
\text { Pusher Prop }\end{array}$ & 20 & 5 & 7.25 & 1 & 85 & 10000 \\
\hline United States & Centurion & Solar & 1300 & 100 & 206 & 14 & 200 & 100000 \\
\hline United States & Dakota & Piston & 208 & 50 & 15.6 & 4.5 & 170 & 12000 \\
\hline United States & Dakota & Piston & 133 & 50 & 12.7 & 3 & 120 & 15000 \\
\hline United States & Dragon Drone & Piston & 91 & 15 & 8.2 & 2 & 48 & 10000 \\
\hline United States & Flyrt & Battery & 72 & 25 & 8 & 0.5 & 4 & 2000 \\
\hline United States* & Global Hawk & Turbofan & 25600 & 1900 & 116.2 & 35 & 12000 & 65000 \\
\hline United States & Gnat & Piston & 1125 & 140 & 35.3 & 40 & 250 & 20000 \\
\hline United States & Hellfox & Rotary & 350 & 50 & 11 & 8 & 54 & 15000 \\
\hline United States* & Hunter II & Turbojet & 2700 & 1000 & 54 & 29 & 620 & 25000 \\
\hline United States & Inventus-e & Electric & 6 & 12 & 6.3 & 2 & 90 & 10000 \\
\hline United States & Inventus-S1 & Piston & 25 & 75 & 12 & 30 & 2000 & 10000 \\
\hline United States & Isis & Rotary & 351 & 75 & 24 & 12 & 1100 & 10000 \\
\hline United States & Javelin & Piston & 15 & 3 & 8 & 2 & 1 & 1000 \\
\hline United States* & Mariner & Turboprop & 11000 & 1150 & 86 & 49 & 40 & 52000 \\
\hline United States & Mini Vanguard & Piston & 100 & 12 & 7 & 3 & 31 & 16300 \\
\hline United States & Neptune & Piston & 130 & 20 & 7 & 3 & 40 & 8000 \\
\hline United States & Outrider & Piston & 500 & 60 & 13 & 6 & 108 & 15000 \\
\hline United States & P-7108/7108V & Piston & 40 & 10 & 8.5 & 2 & 5 & 5000 \\
\hline United States & Pathfinder & Solar/Propeller & 480 & 25 & 100 & 14 & 108 & 70000 \\
\hline United States* & Perseus B & Piston & 2500 & 110 & 65 & 6 & 200 & 65000 \\
\hline United States & Pioneer (RQ-2A) & Rotary & 450 & 100 & 16.9 & 5.5 & 100 & 15000 \\
\hline United States & Pointer (FQM-151A) & Battery & 8 & 2 & 9 & 1.5 & 9 & 1000 \\
\hline United States & Porter & Piston & 200 & 50 & 12 & 4 & 27 & 5000 \\
\hline United States & Prowler I & Rotary & 200 & 50 & 18 & 6 & 140 & 21000 \\
\hline
\end{tabular}




\begin{tabular}{|c|c|c|c|c|c|c|c|c|}
\hline Country & Designation & Propulsion & $\begin{array}{l}\text { Gross } \\
\text { (lb.) }\end{array}$ & $\begin{array}{l}\text { Payload } \\
\text { (Ib.) }\end{array}$ & $\begin{array}{c}\text { Wingspan } \\
\text { (ft.) }\end{array}$ & $\begin{array}{c}\text { Endurance } \\
\text { (h.) }\end{array}$ & $\begin{array}{l}\text { Range } \\
\text { (n. mi.) }\end{array}$ & $\begin{array}{c}\text { Ceiling } \\
\text { (ft.) }\end{array}$ \\
\hline United States & Prowler II & Piston & 650 & 100 & 24 & 12 & 135 & 20000 \\
\hline United States & R4E SkyEye & Rotary & 1250 & 300 & 24 & 12 & 360 & 16000 \\
\hline United States* & Raptor & Piston & 1764 & 75 & 65.8 & 24 & 150 & 65000 \\
\hline United States & Scarab & Turbojet & 2370 & 290 & 11 & 2 & 500 & 43000 \\
\hline United States & Sea Ferret & Turbojet & 150 & 30 & 6 & 3 & 160 & 20000 \\
\hline United States & SeaScan & $\begin{array}{l}\text { Pusher-Prop } \\
\text { Piston }\end{array}$ & 38.4 & 1.3 & 9.8 & 15 & 900 & 23000 \\
\hline United States & Sender & Battery & 10 & 3 & 4 & 2 & 50 & 5000 \\
\hline United States & Shadow 200 & Rotary & 375 & 55.7 & 14 & 6 & 108 & 19000 \\
\hline United States & Shadow 200T & Piston & 280 & 40 & 14 & 4 & 27 & 19000 \\
\hline United States & Shadow 600 & Rotary & 583 & 90.2 & 22.4 & 14 & 108 & 16000 \\
\hline United States* & Skywatch & Turboprop & 7900 & 900 & 114 & 32 & 250 & 62000 \\
\hline United States & SLURS & Battery & 10 & 2 & 5 & 1 & 5 & 500 \\
\hline United States & Starbird & Rotary & 380 & 50 & 17.3 & 4 & 108 & 17000 \\
\hline United States & Swallow & Battery & 62 & 10 & 15 & 2 & 60 & 30000 \\
\hline United States & Tern & Piston & 125 & 25 & 11.7 & 4 & 200 & 10000 \\
\hline United States & TS-2000 & Piston & 1000 & 400 & 27 & 4 & 27 & 5000 \\
\hline United States & Vixen & Rotary & 200 & 50 & 9.2 & 4 & 50 & 15000 \\
\hline
\end{tabular}




\section{APPENDIX B: Greedy Algorithm Matlab Code}

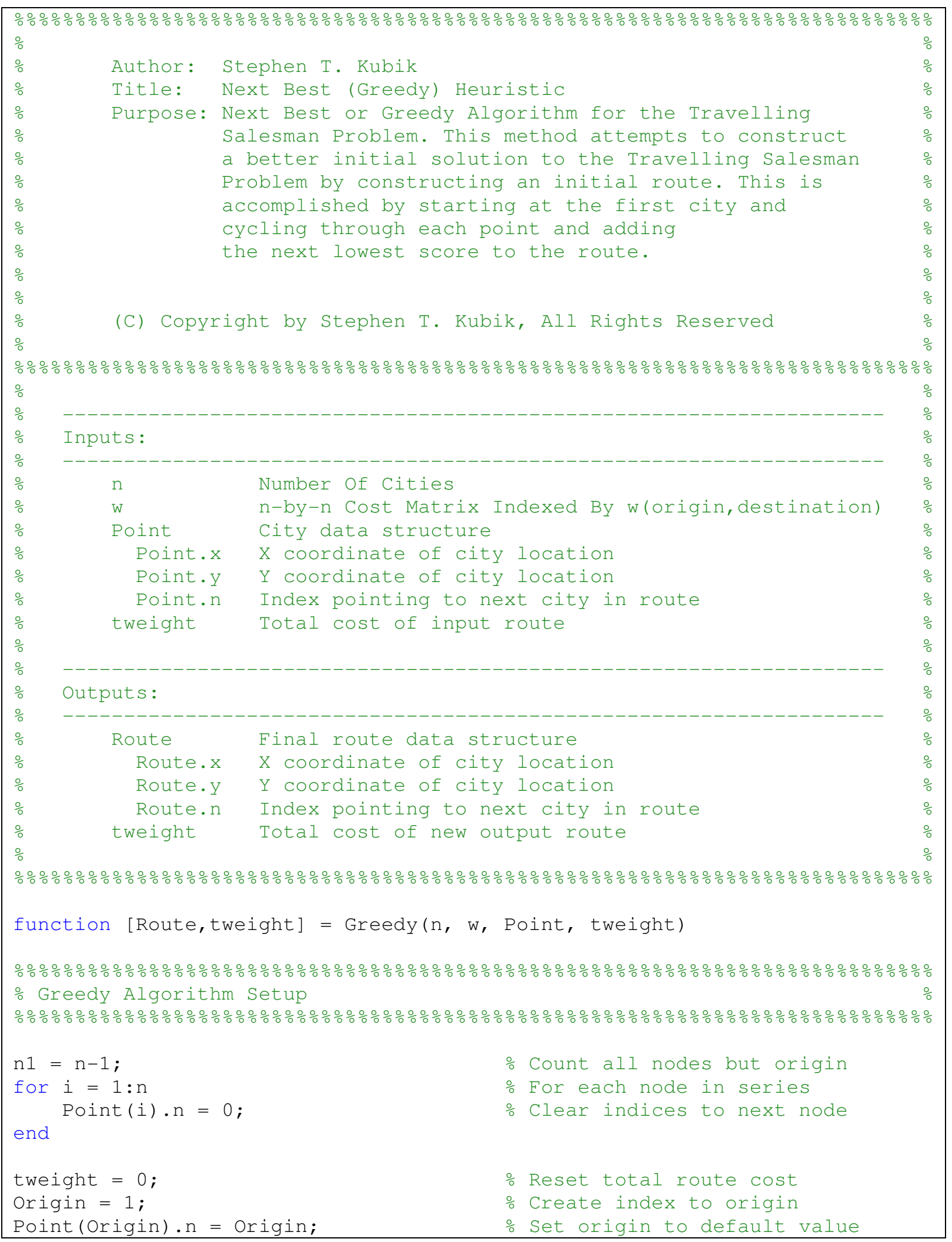




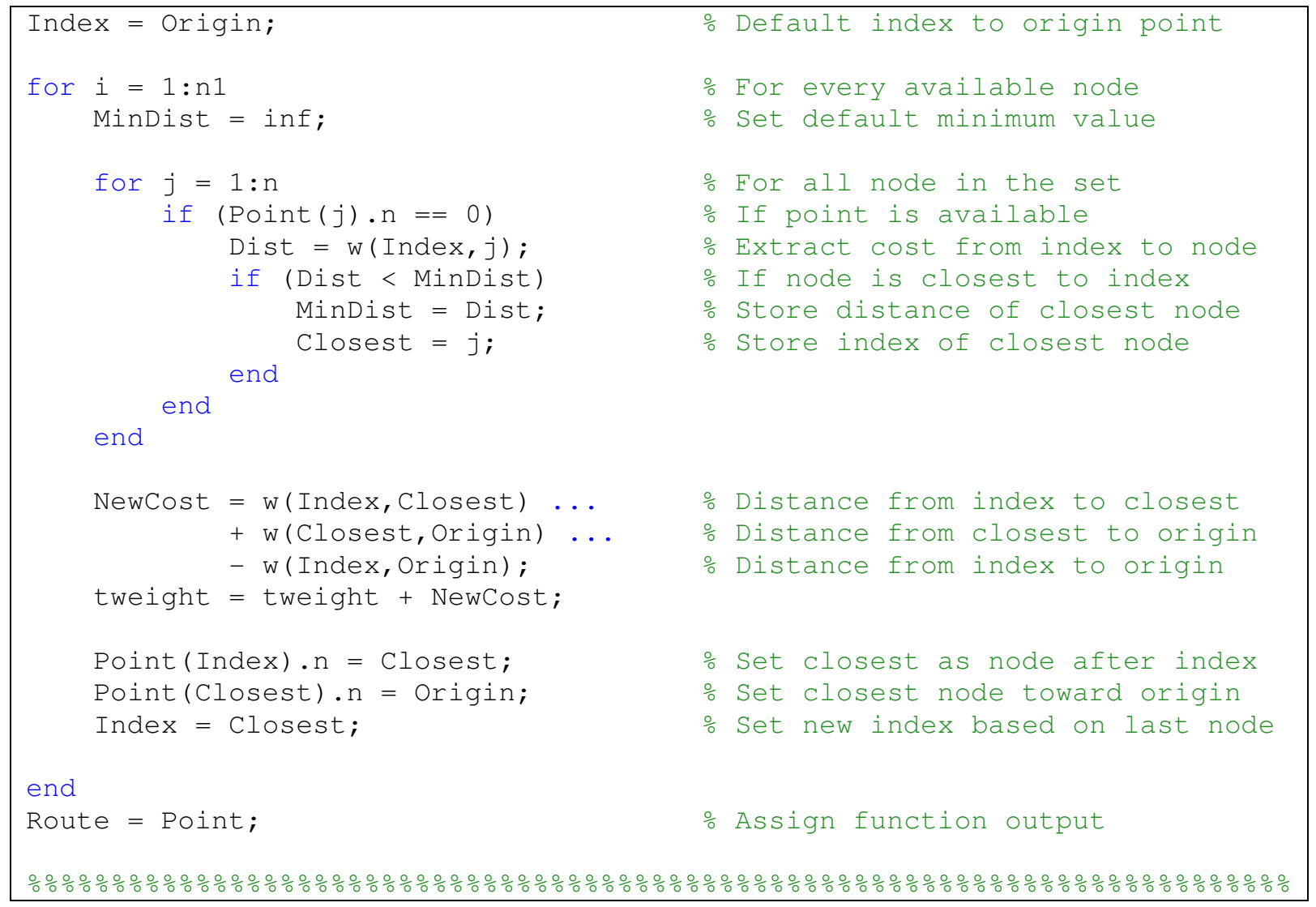




\section{APPENDIX C: Insert Furthest Algorithm Matlab Code}

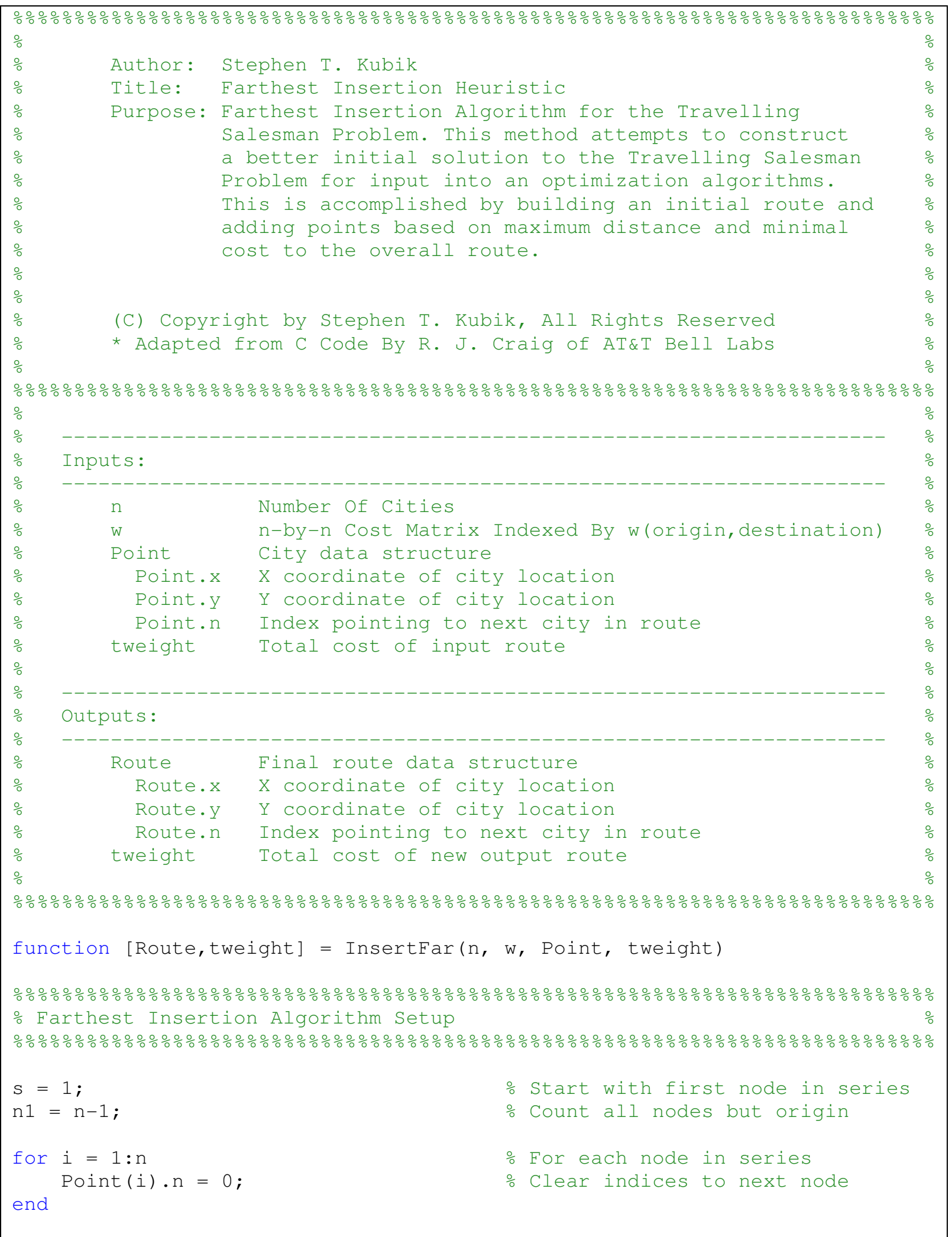




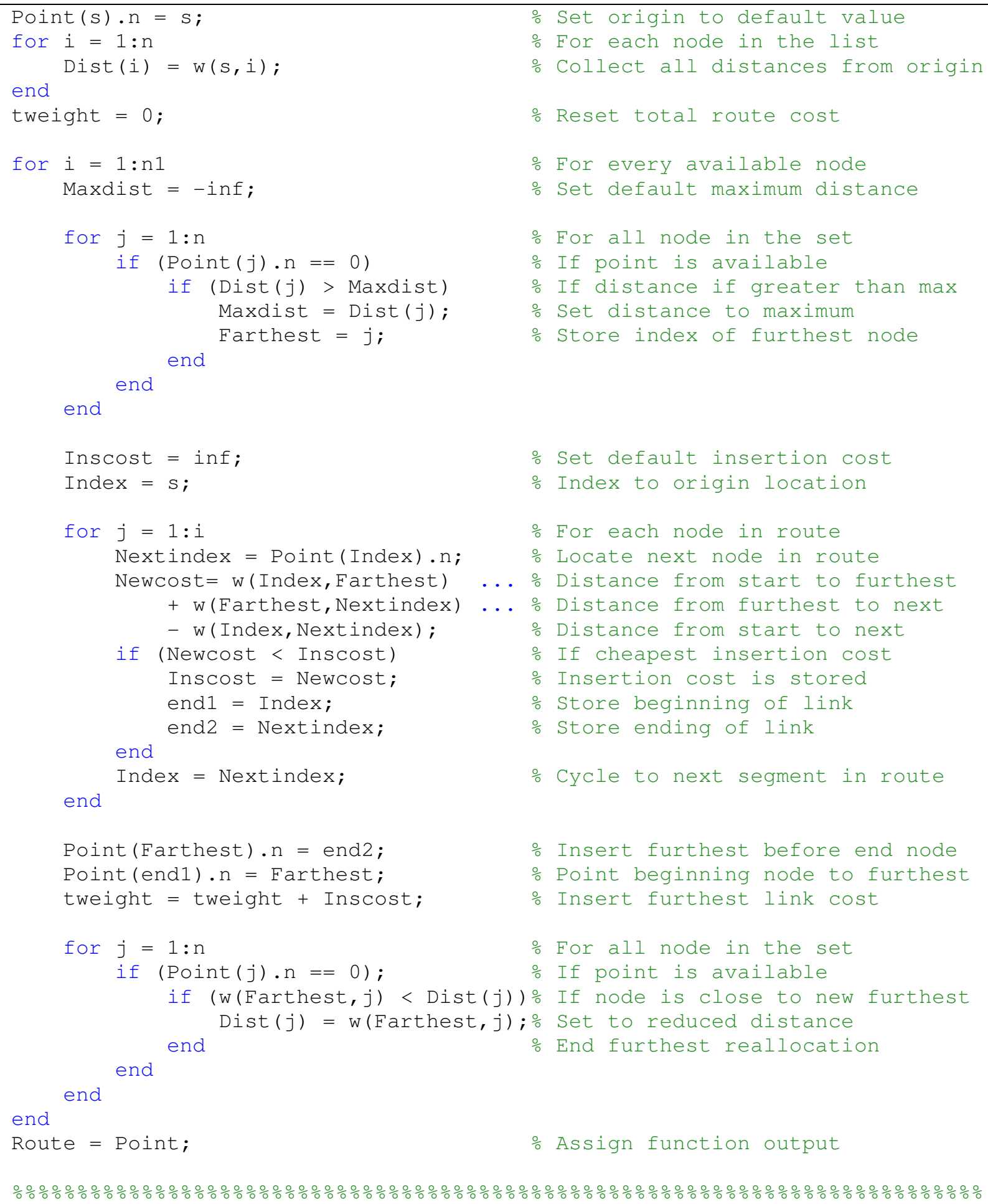




\section{APPENDIX D: Insert Closest Matlab Code}

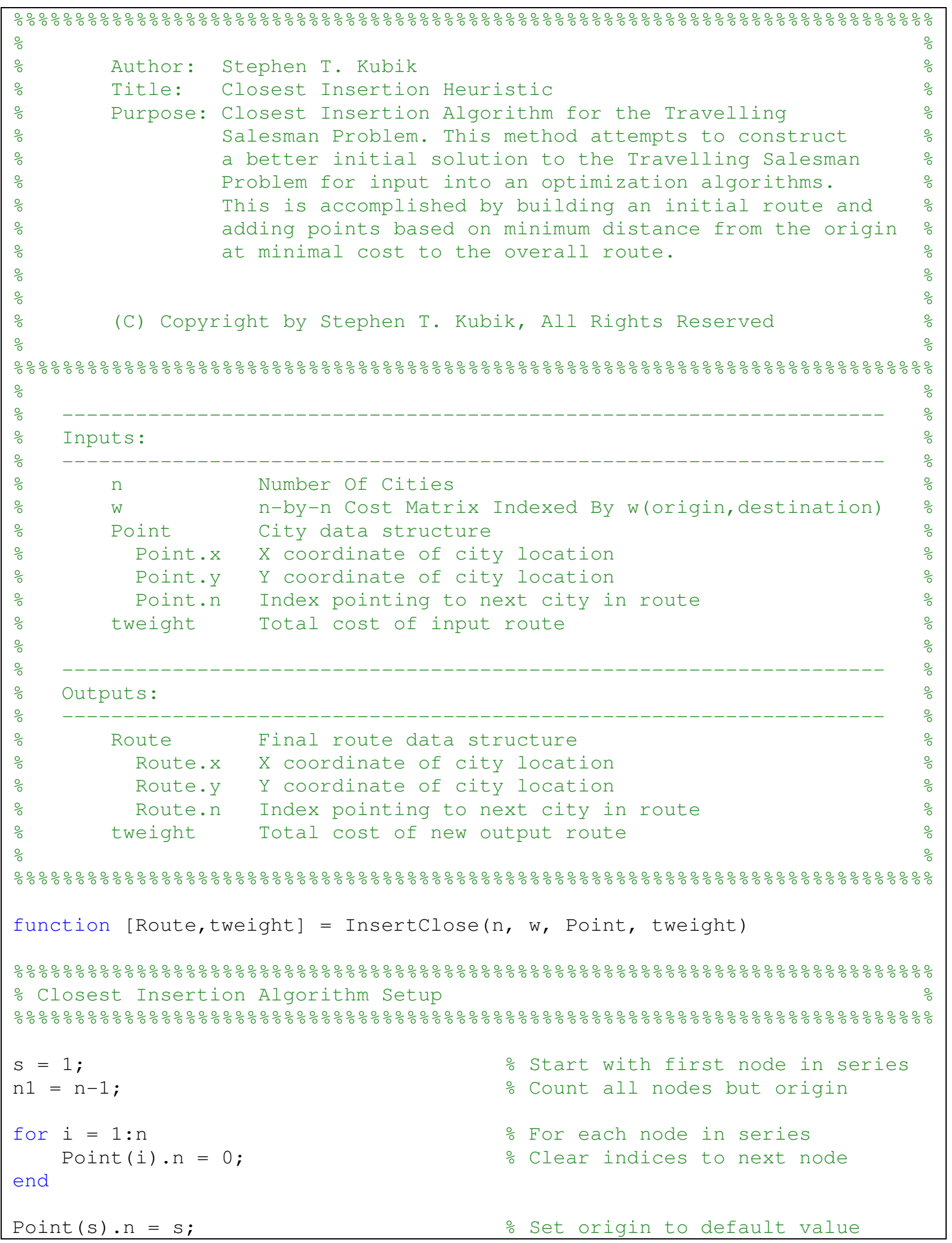




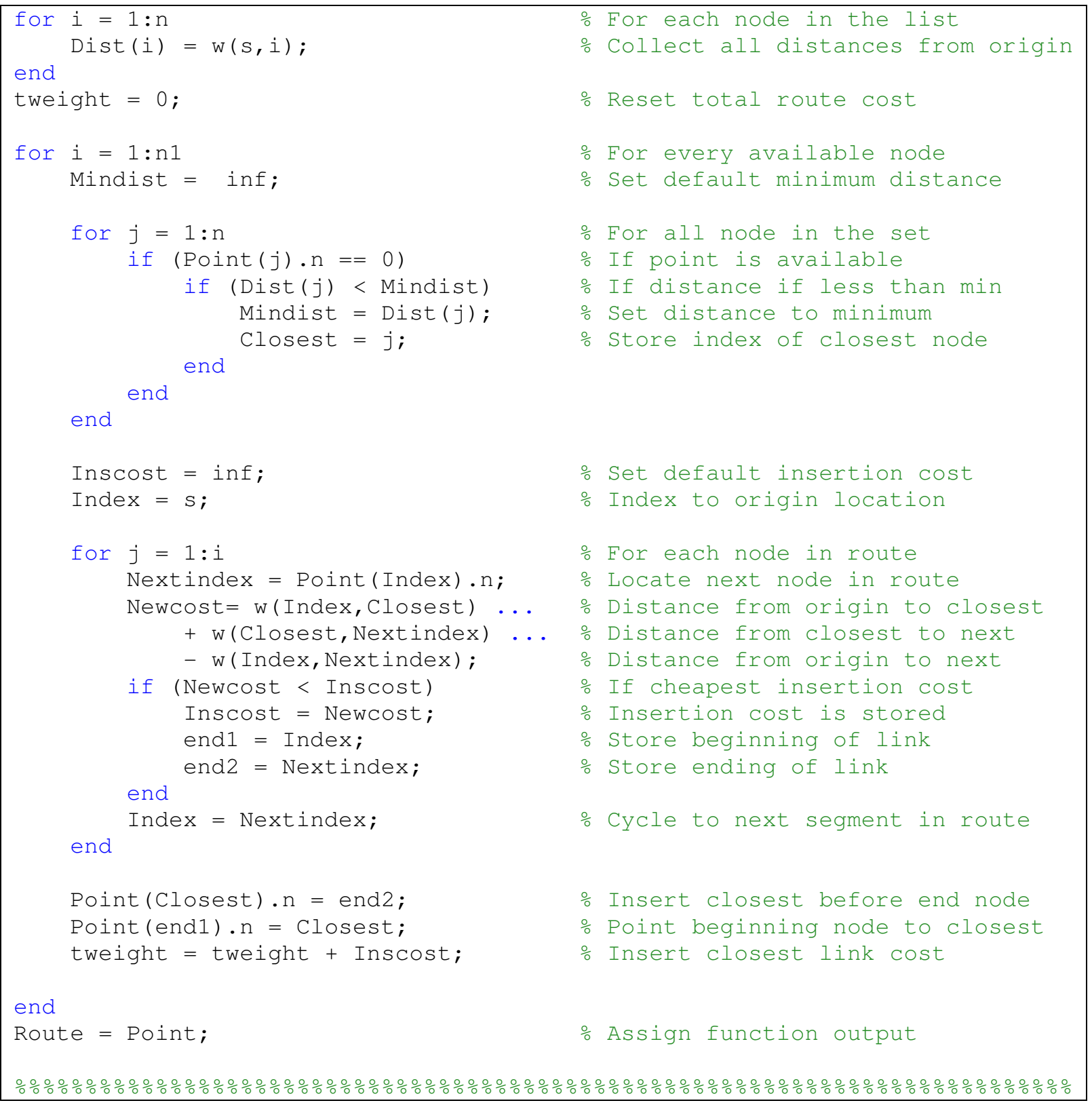




\section{APPENDIX E: 2-Opt Algorithm Matlab Code}

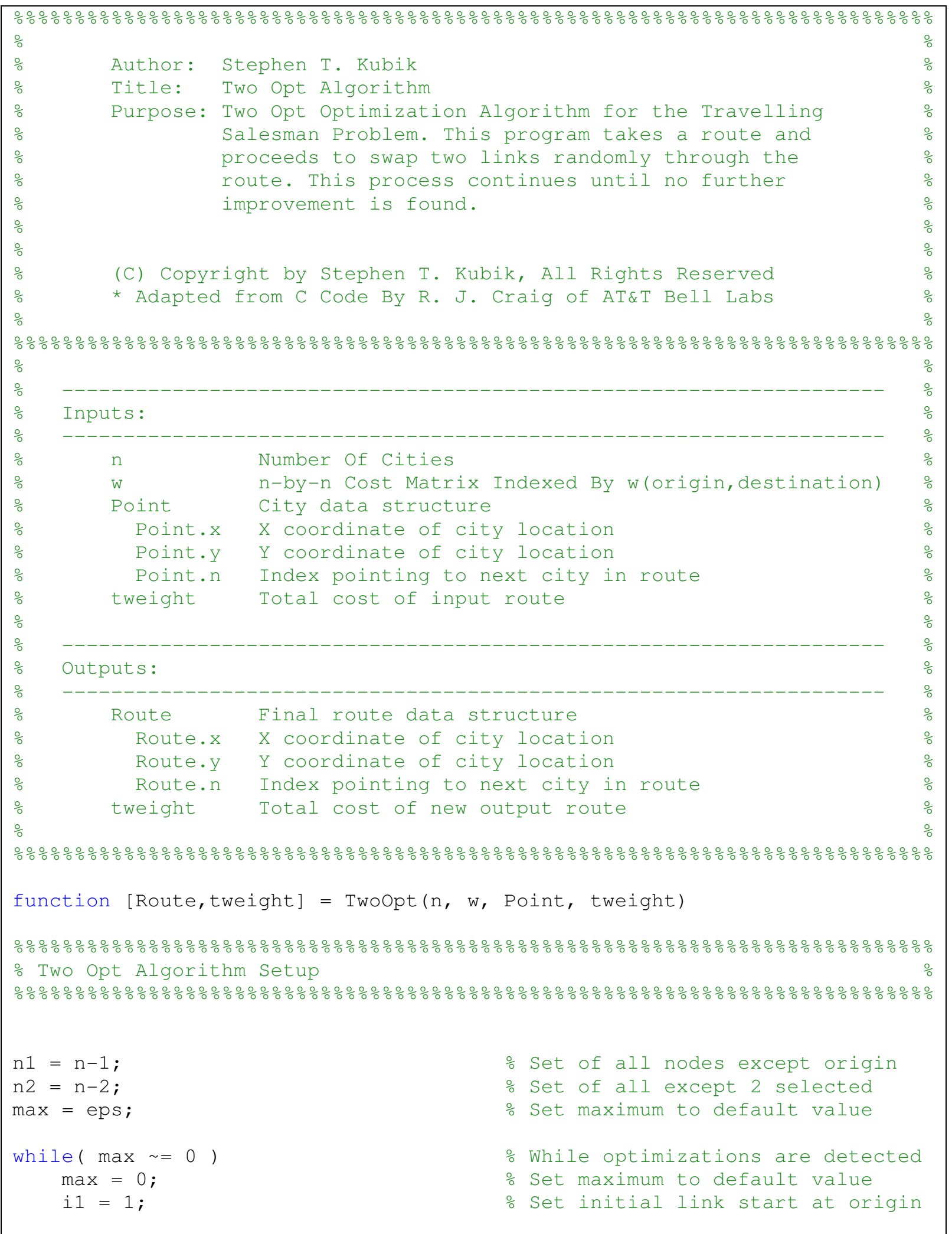




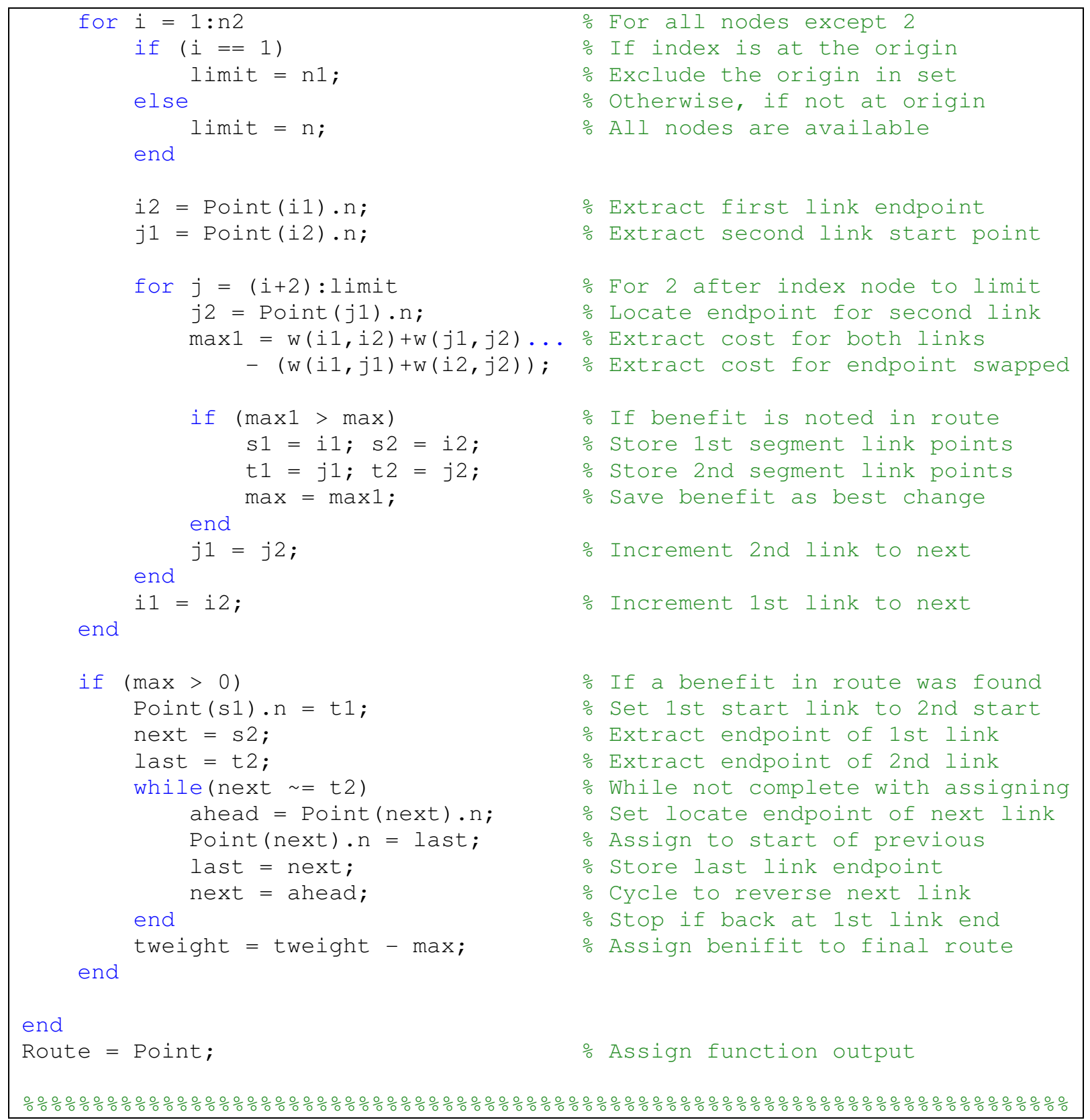




\section{APPENDIX F: 3-Opt Algorithm Matlab Code}

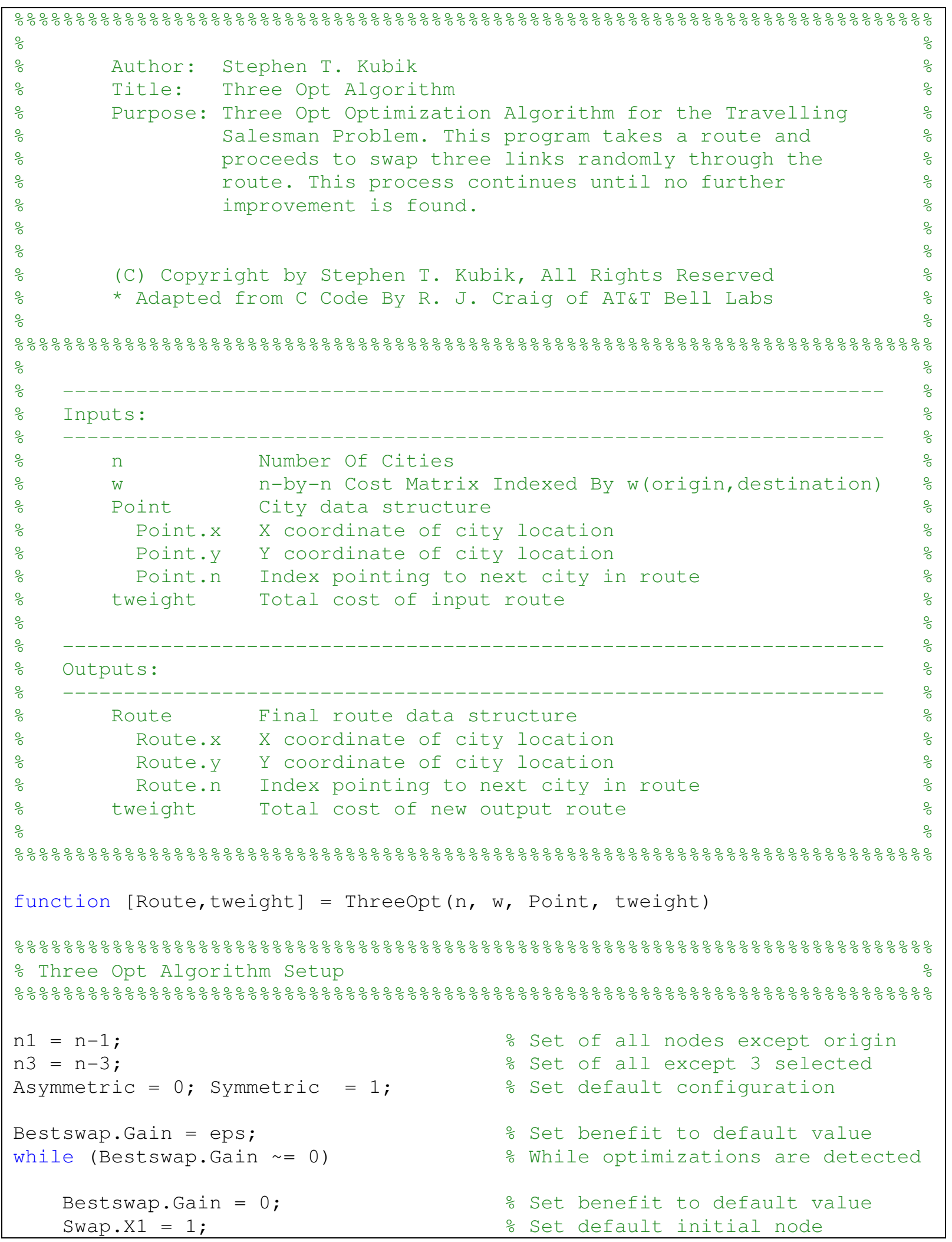




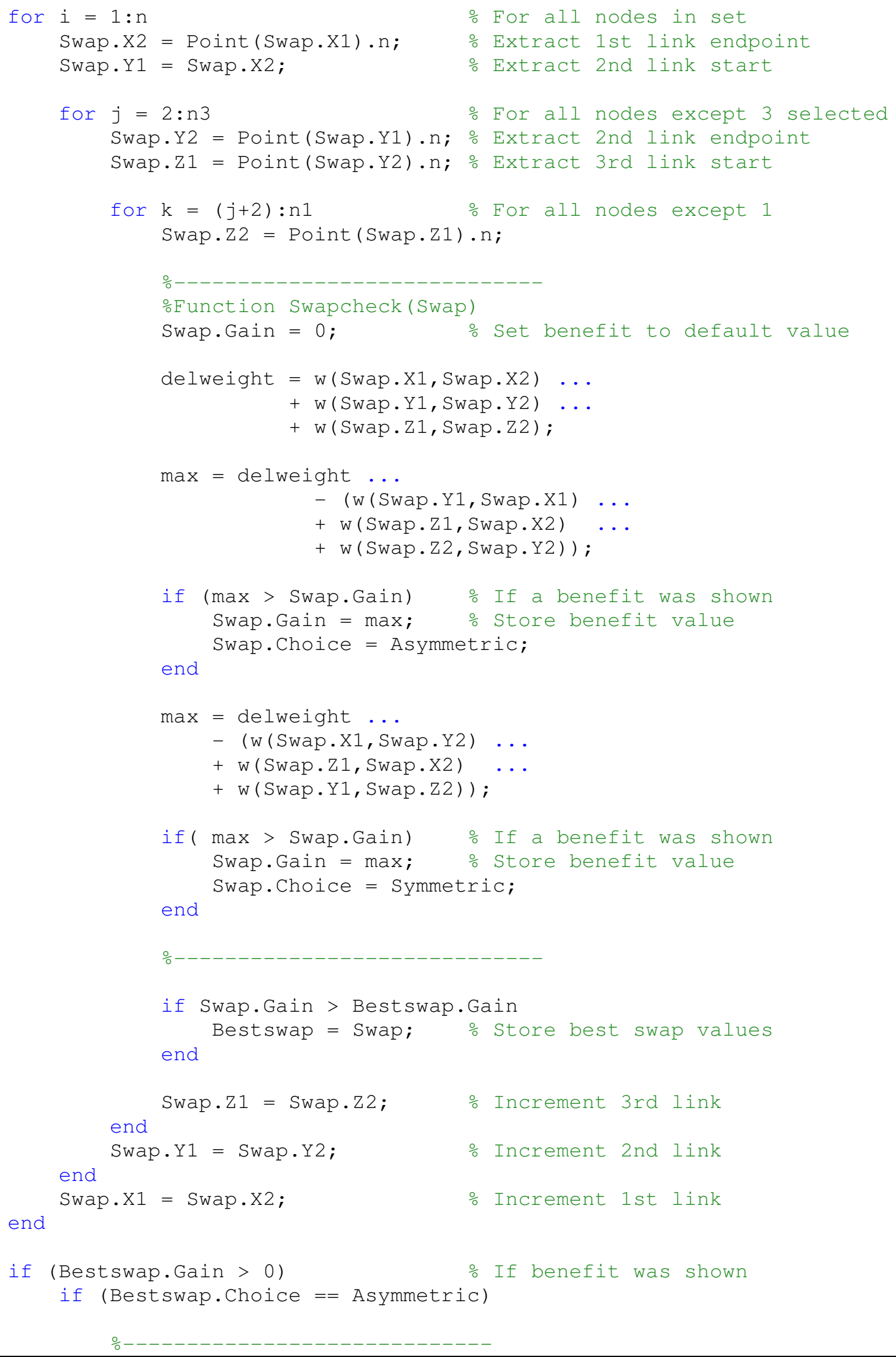




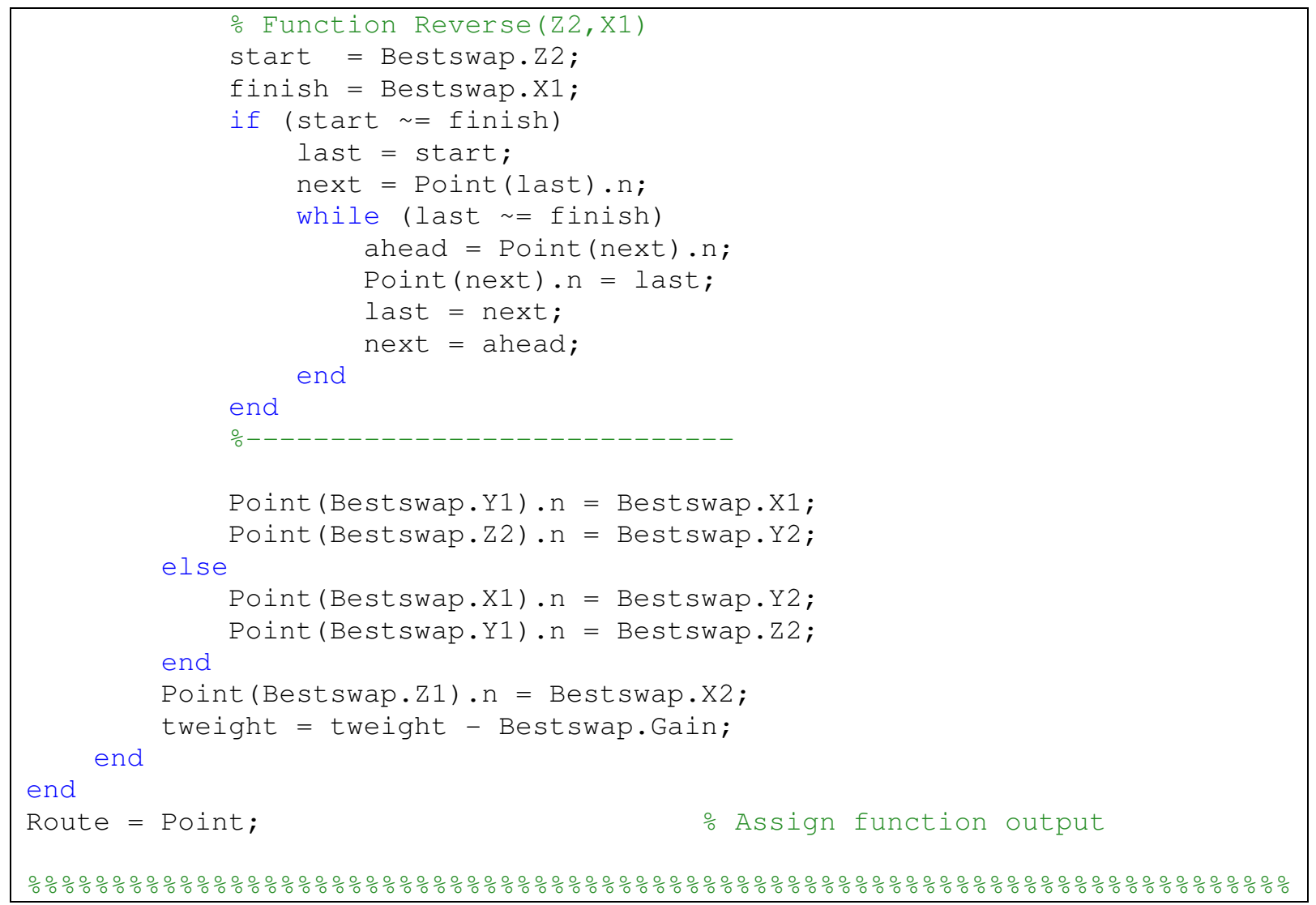




\section{APPENDIX G: LASE Matlab Simulation Code}

LASE Code: Load_LASE_Scenario.m

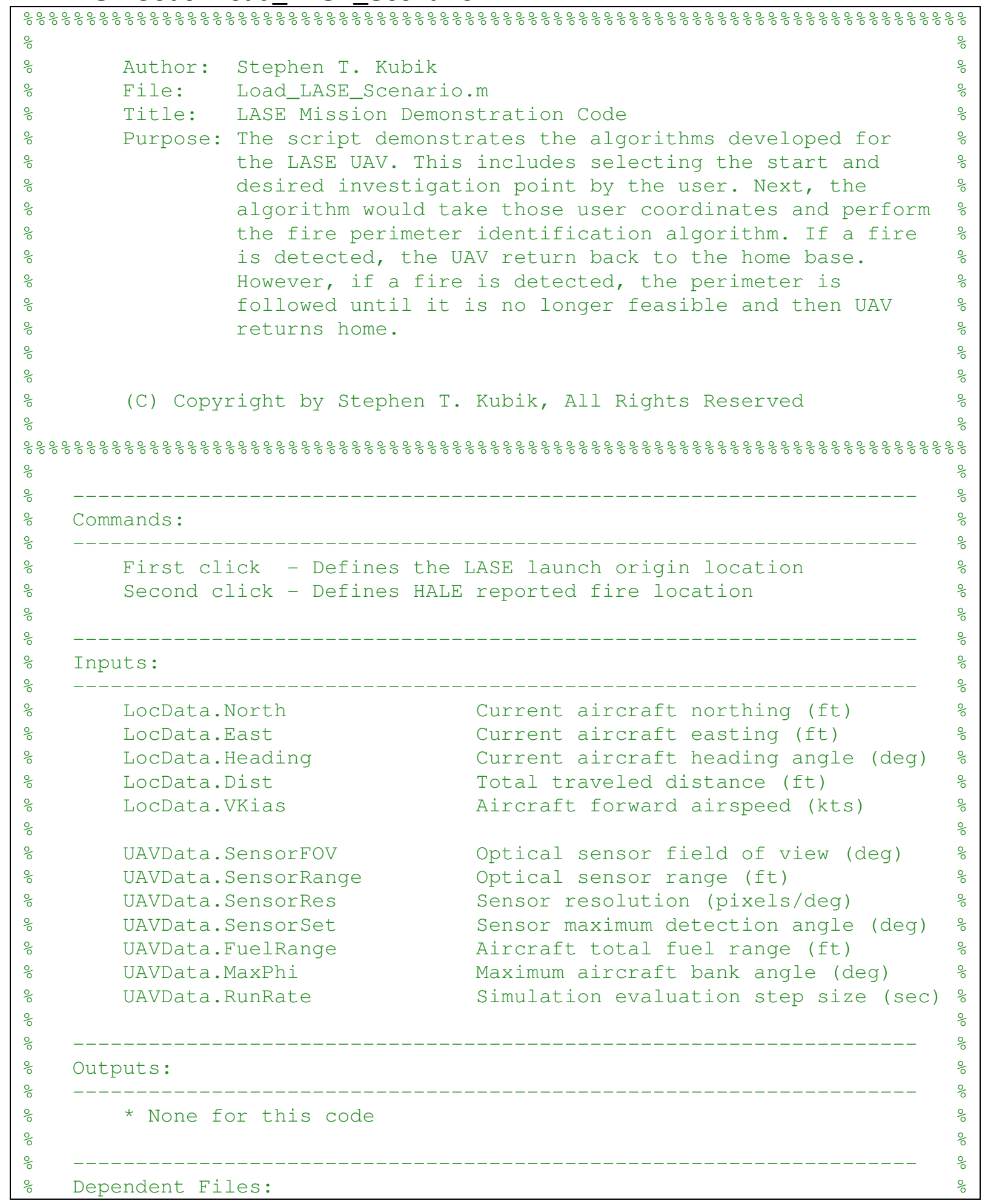




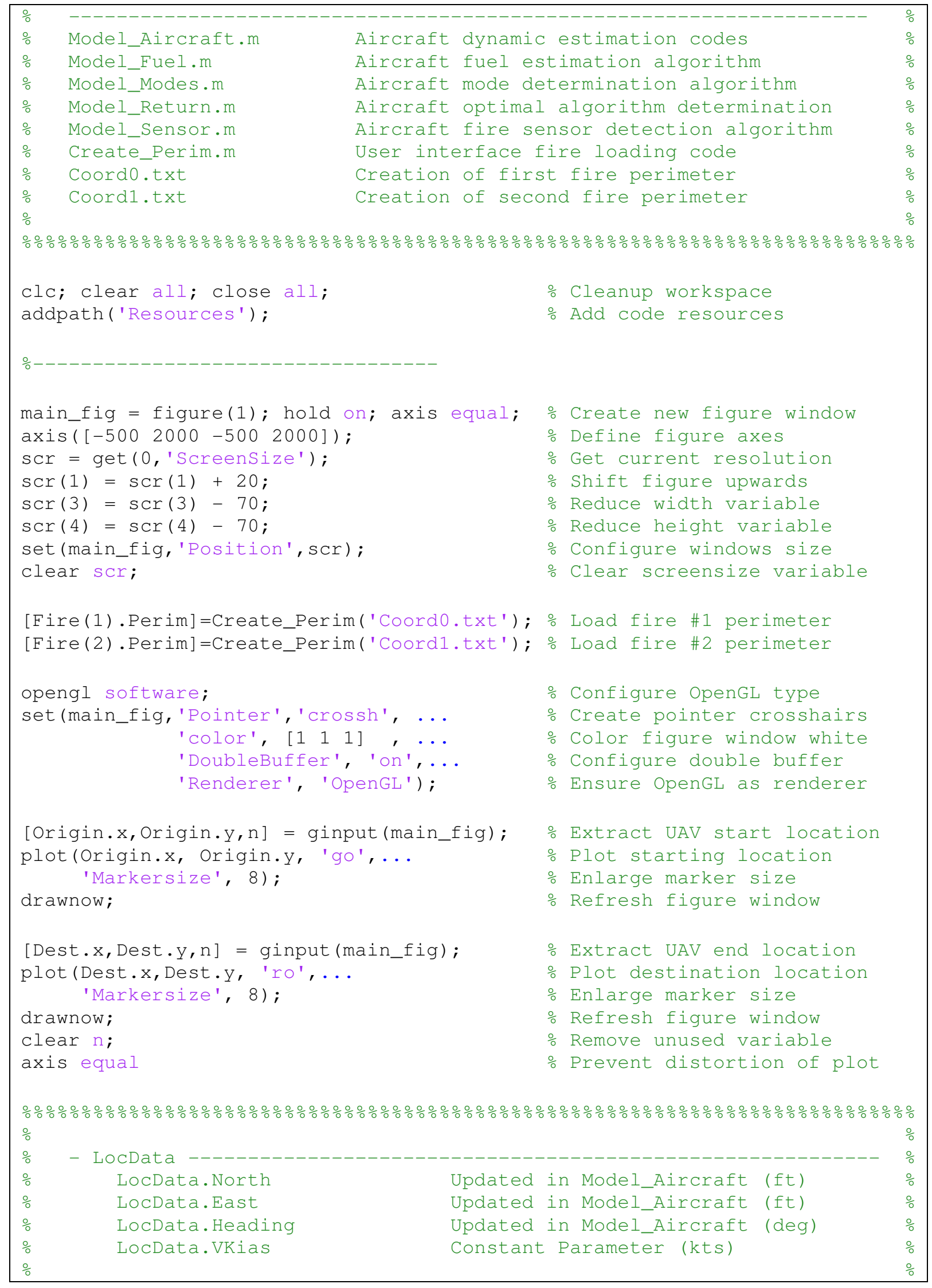




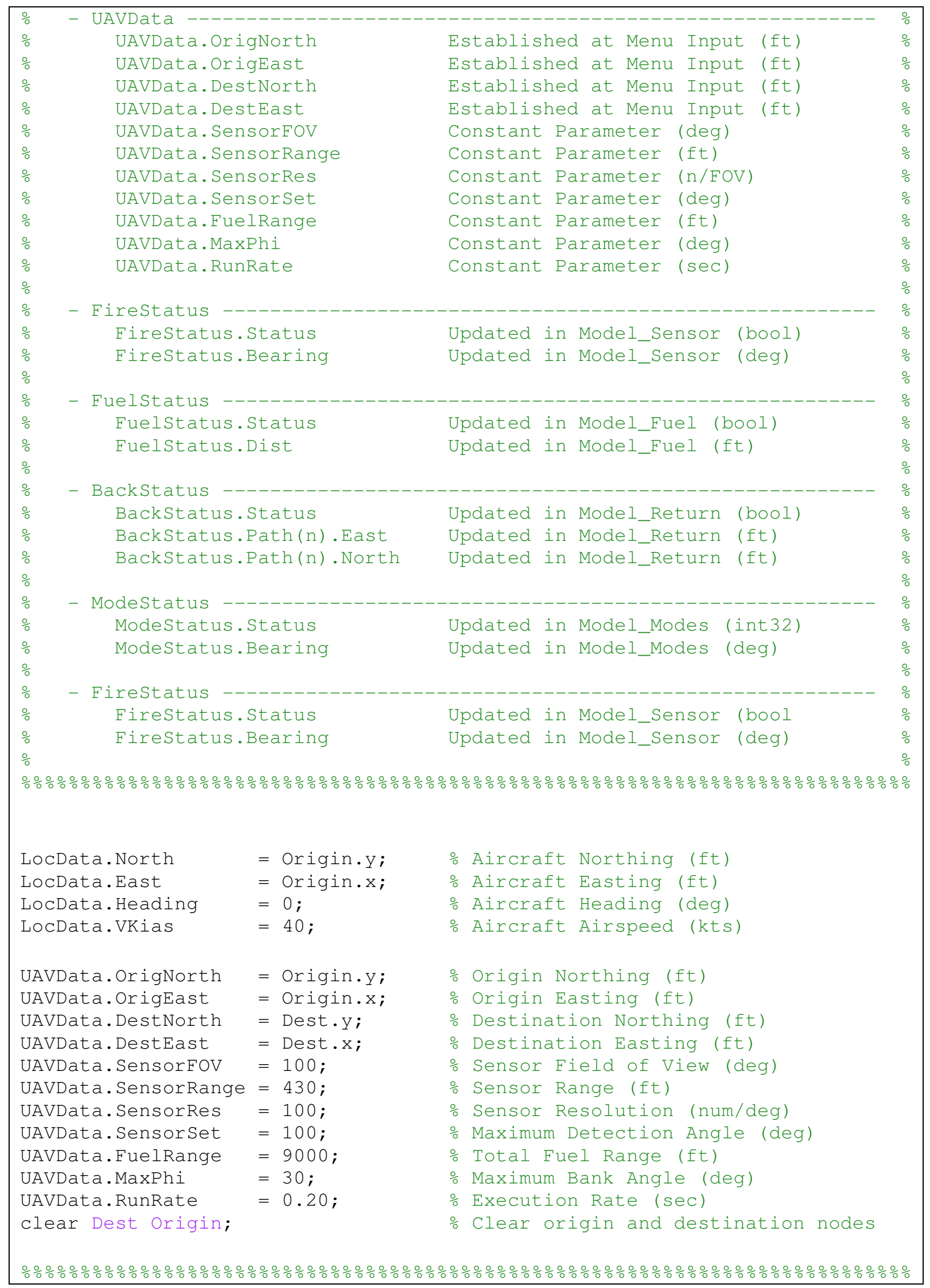




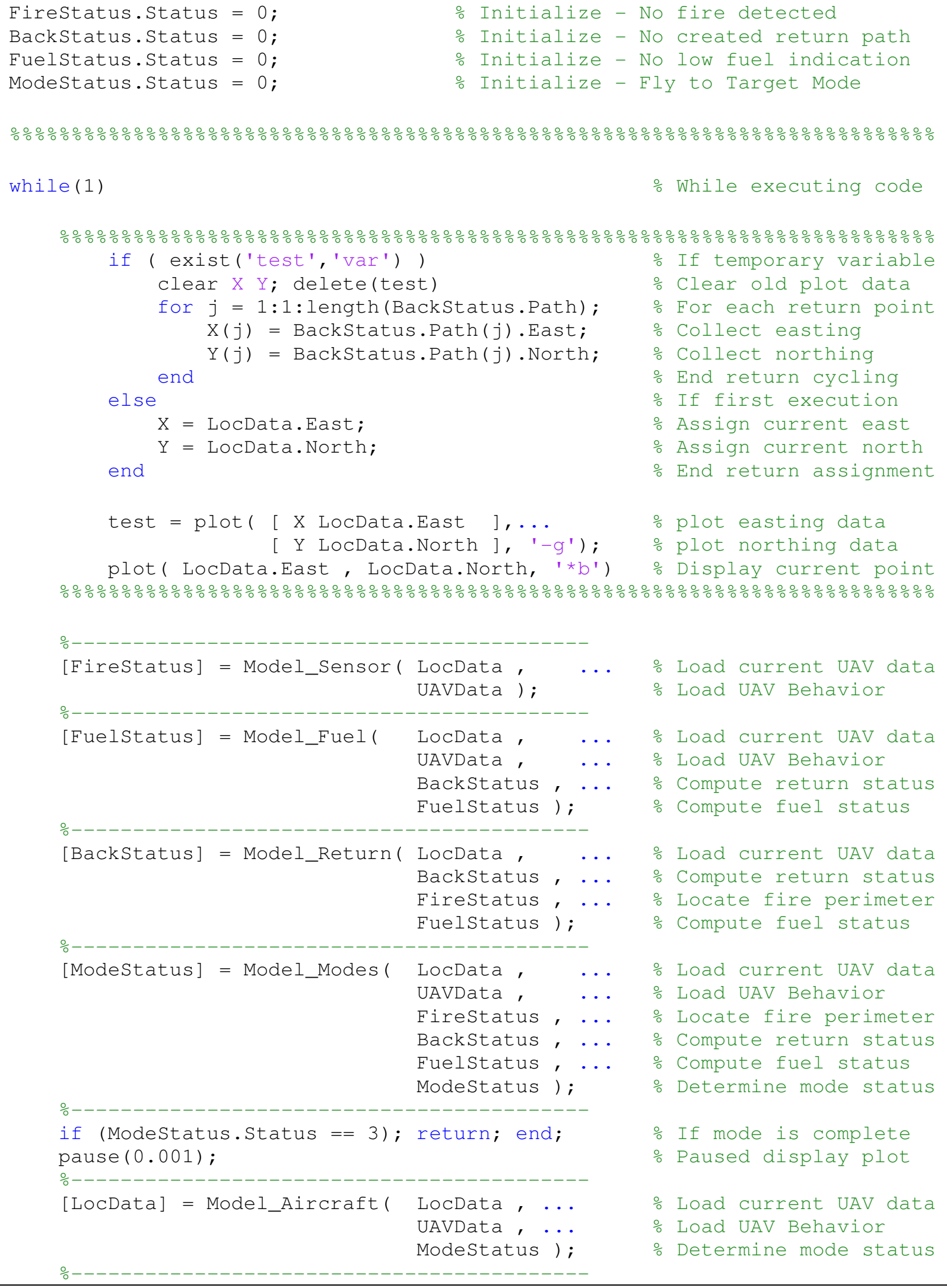




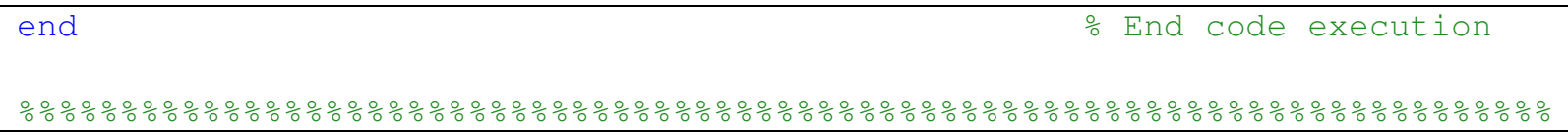

\section{LASE Code: Model_Aircraft.m}

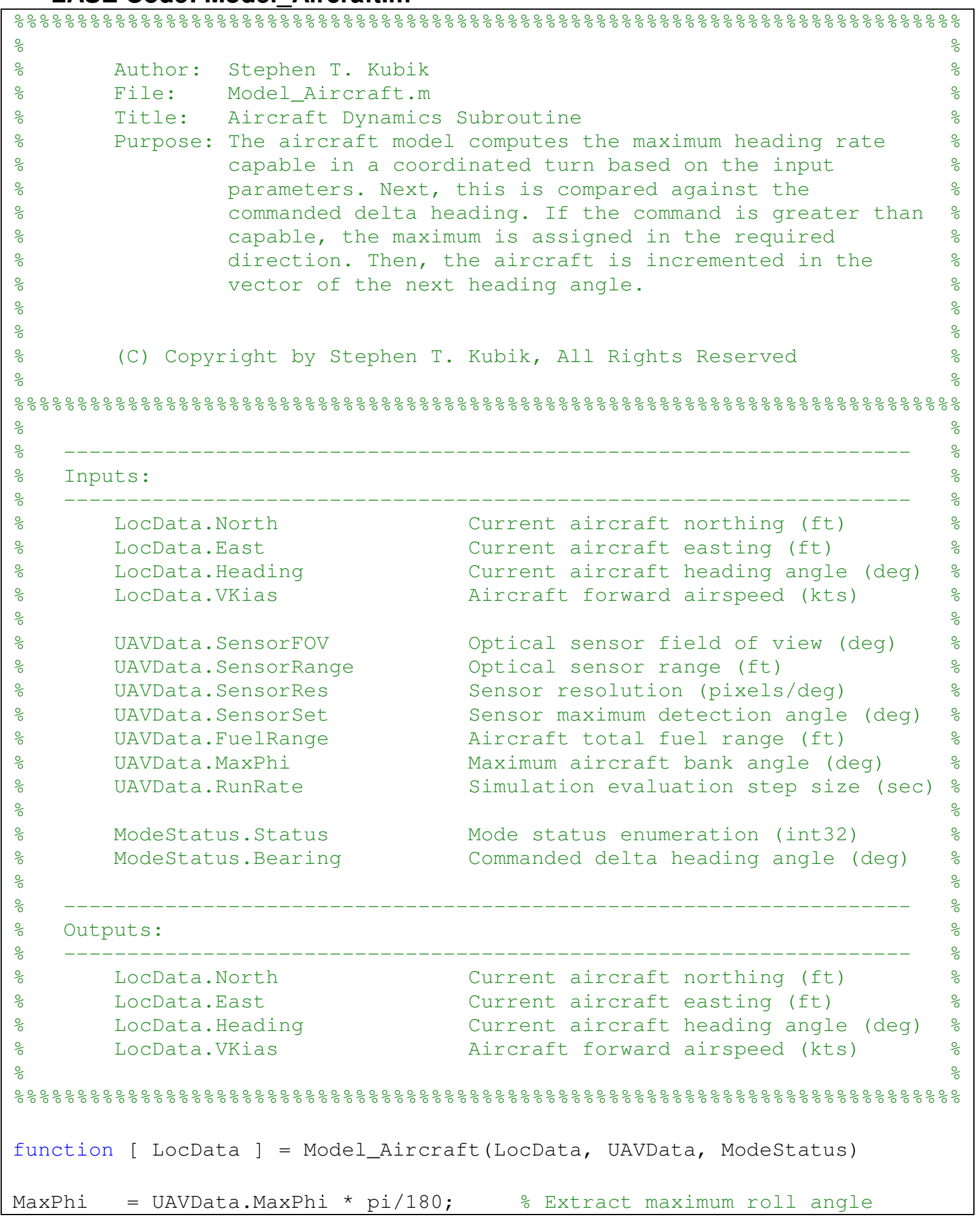




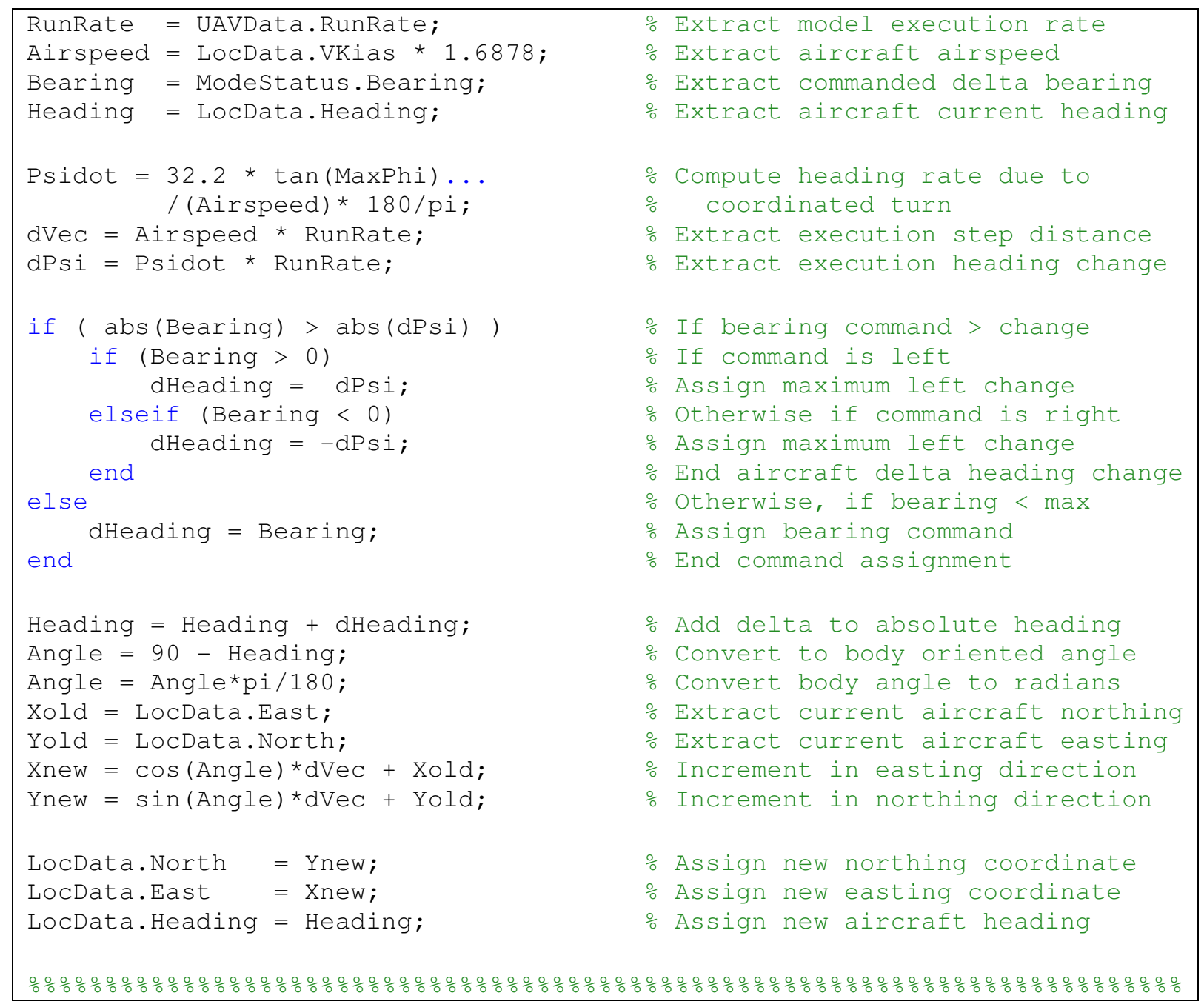

\section{LASE Code: Model Fuel.m}

\begin{tabular}{|c|c|c|c|}
\hline \multicolumn{4}{|c|}{ 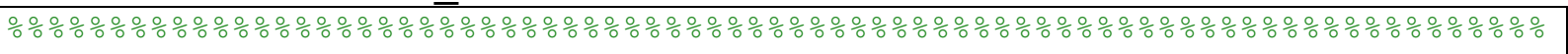 } \\
\hline \% & & & \% \\
\hline$\%$ & Author: & Stephen T. Kubik & $\%$ \\
\hline$\%$ & File: & Model_Fuel.m & $\%$ \\
\hline \% & Title: & Aircraft Fuel Estimation Subroutine & $\%$ \\
\hline \% & Purpose: & The purpose of the algorithm is to compute total fuel & $\%$ \\
\hline$\%$ & & utilized by the aircraft. Then, depending on the mode, & $\%$ \\
\hline \% & & the aircraft will determine the amount of fuel required & 잉 \\
\hline 응 & & to return home. If this total estimated return distance & \% \\
\hline$\%$ & & is equal to the total fuel allocation, the flag will & $\%$ \\
\hline \% & & trigger to send the vehicle to the launch location. & 응 \\
\hline \multicolumn{3}{|r|}{ 然 } & 응 \\
\hline \multicolumn{3}{|r|}{ 然 } & $\%$ \\
\hline$\%$ & \multirow{2}{*}{\multicolumn{2}{|c|}{ (C) Copyright by Stephen T. Kubik, All Rights Reserved }} & $\%$ \\
\hline \% & & & $\frac{\circ}{\circ}$ \\
\hline \multirow{2}{*}{\multicolumn{4}{|c|}{ 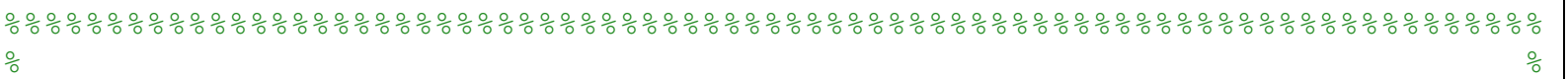 }} \\
\hline & & & \\
\hline 응 & & & \% \\
\hline$\%$ & ats: & & $\%$ \\
\hline
\end{tabular}




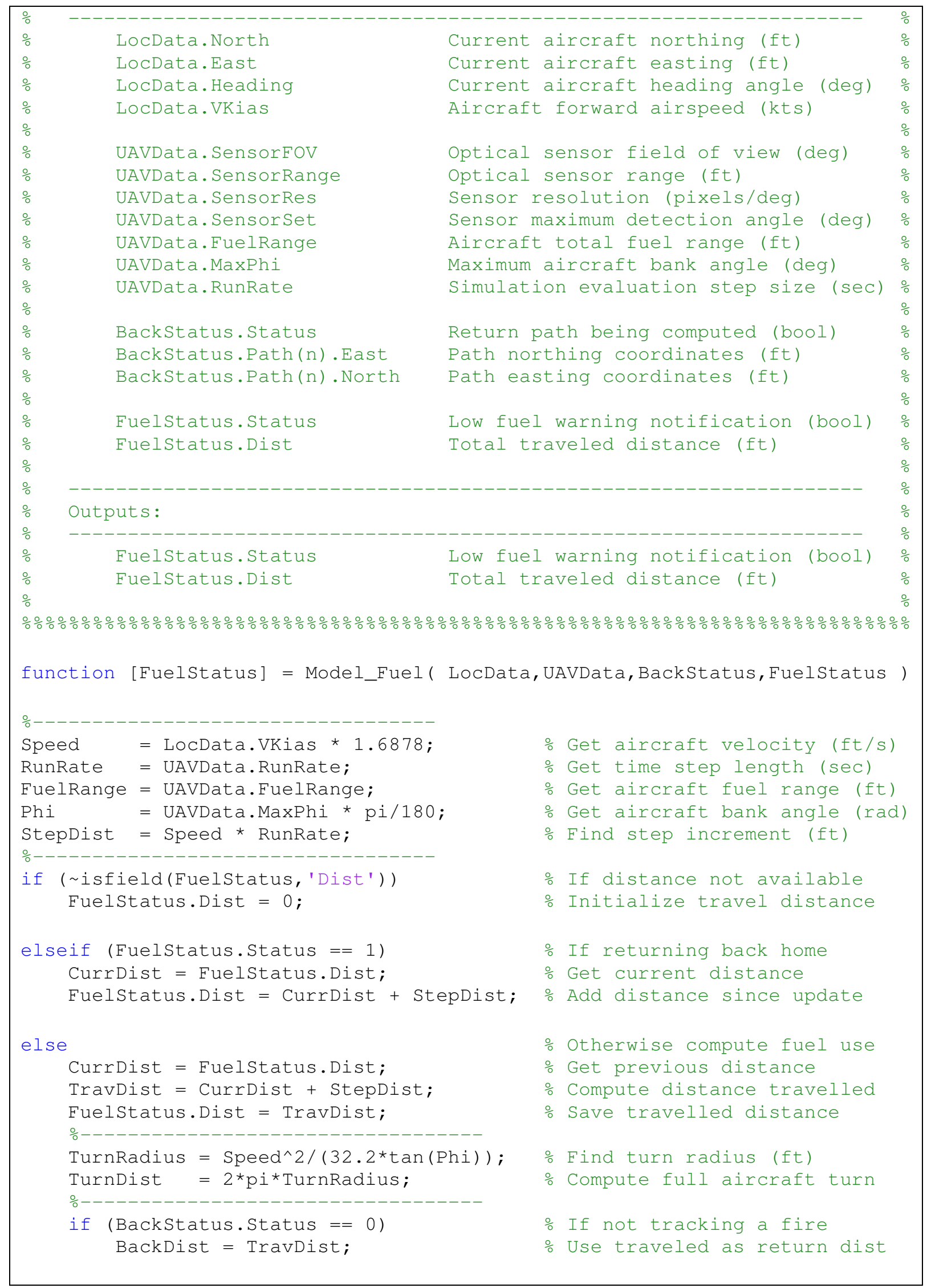




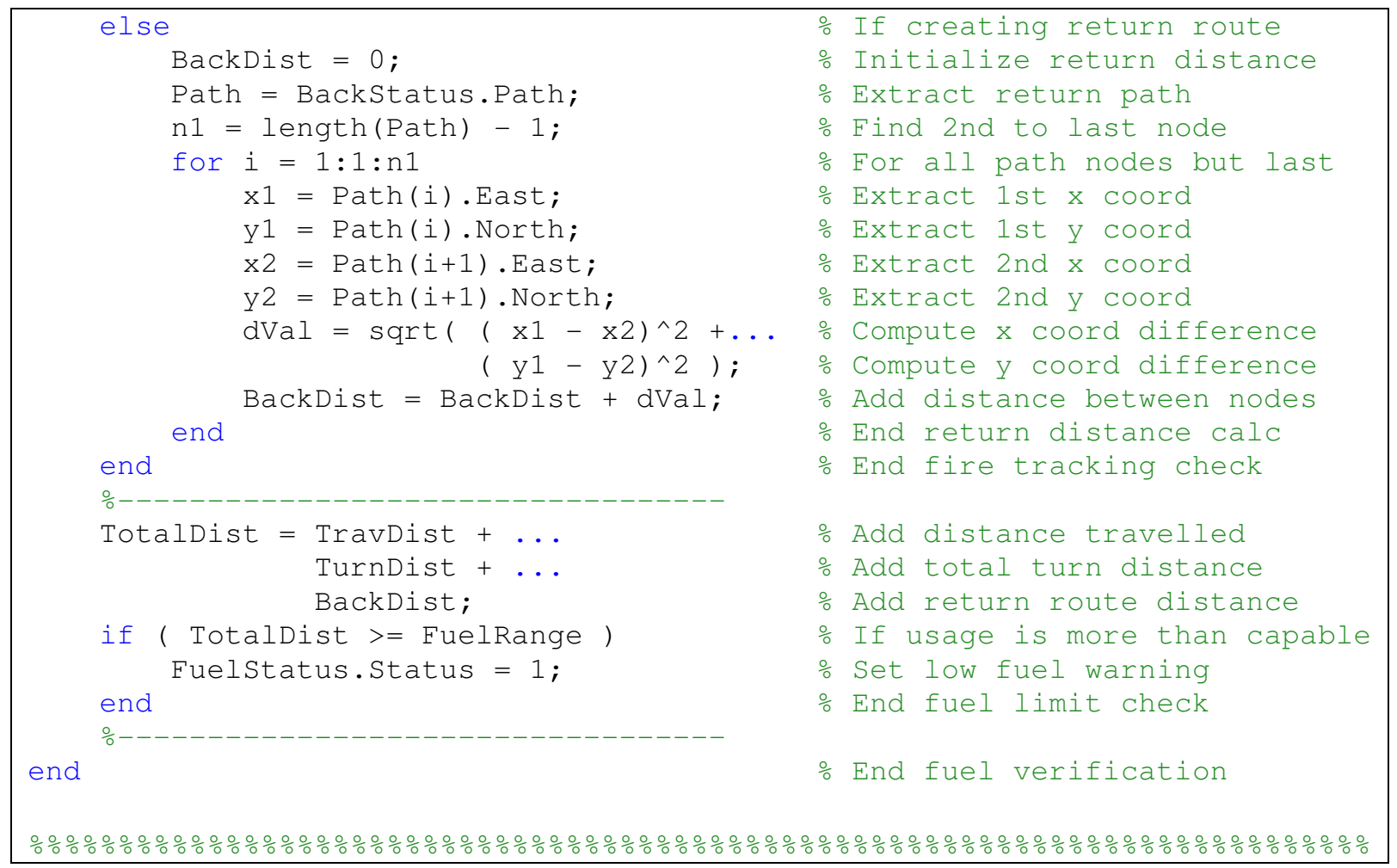

\section{LASE Code: Model Modes.m}

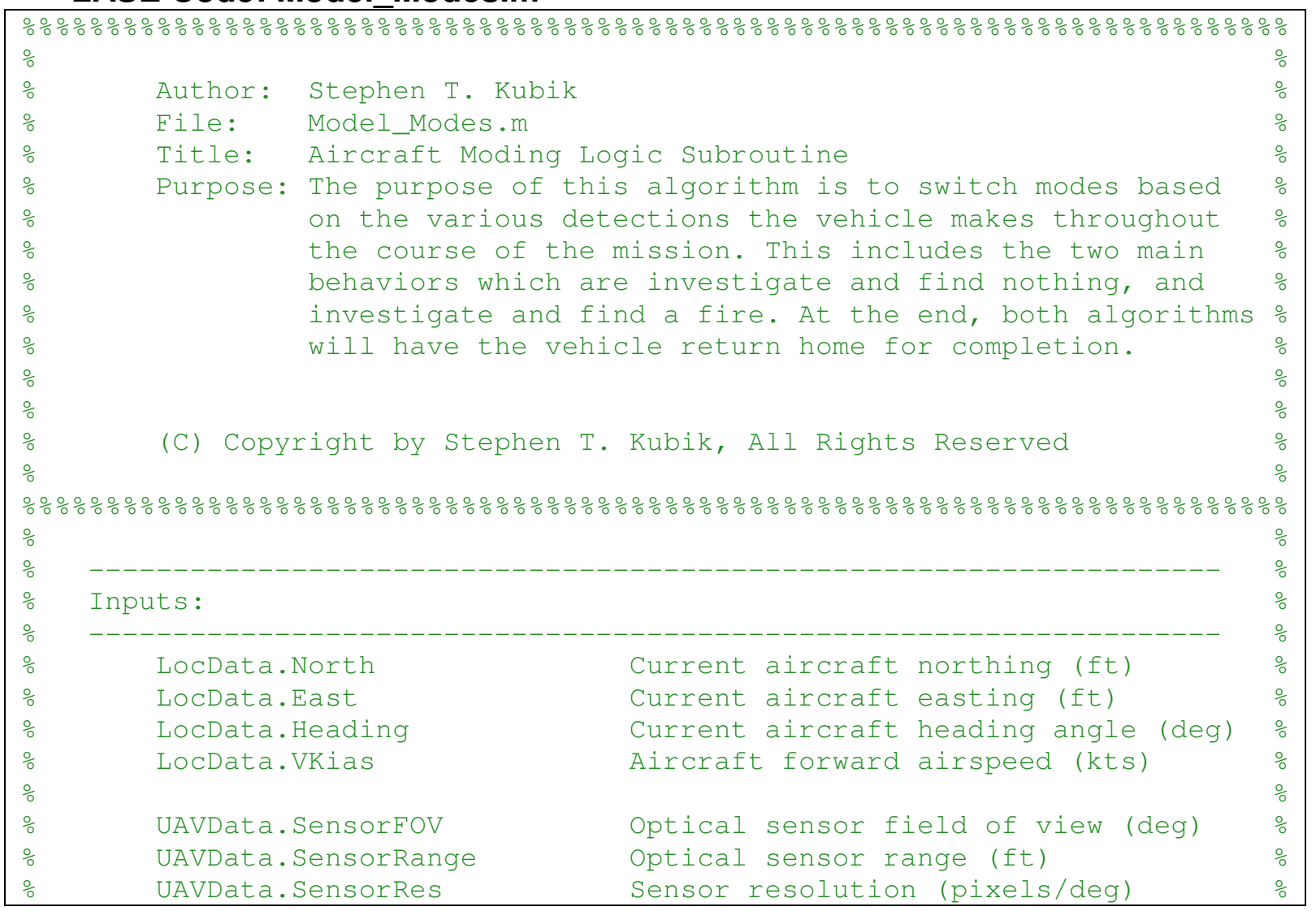




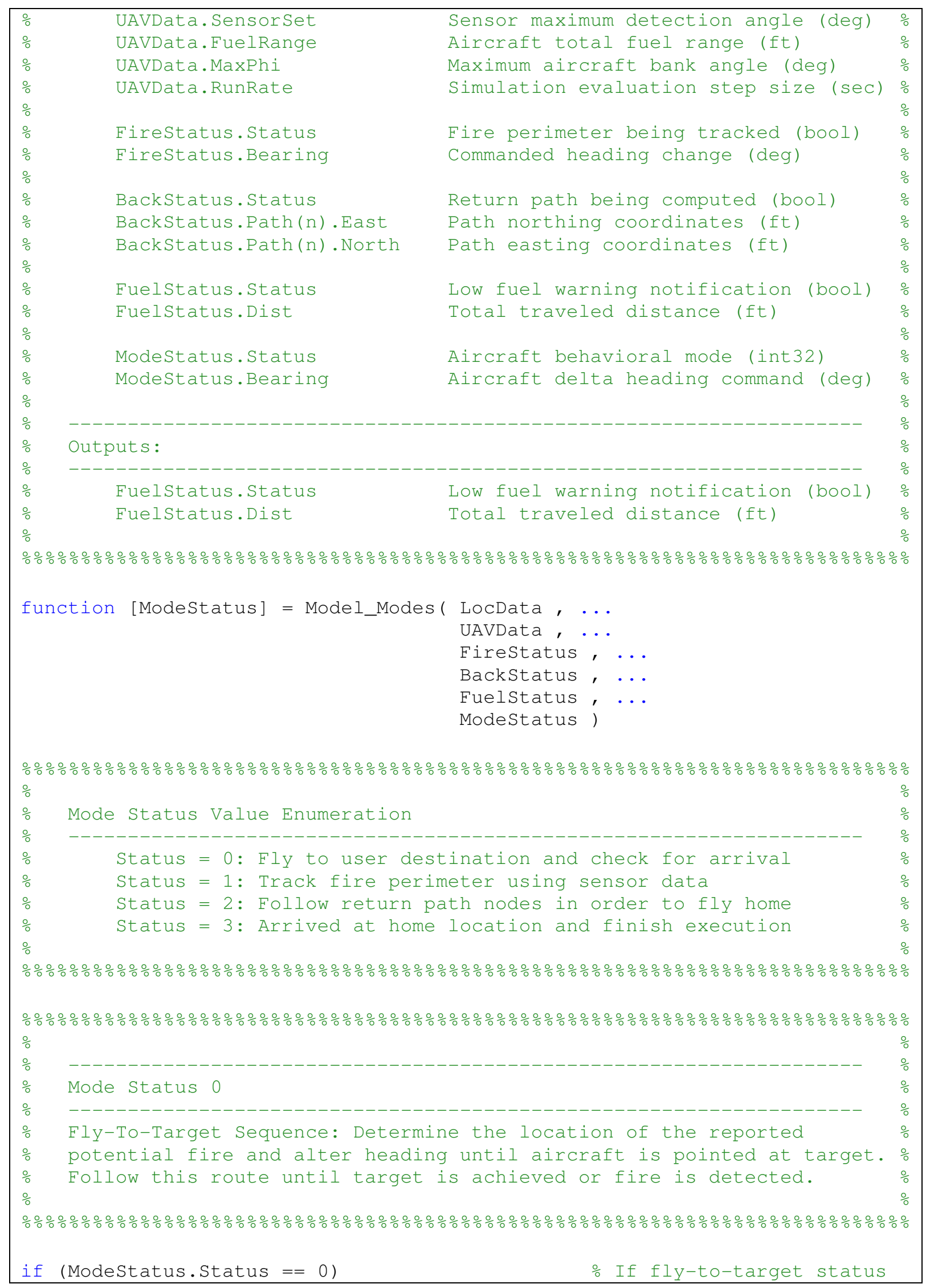




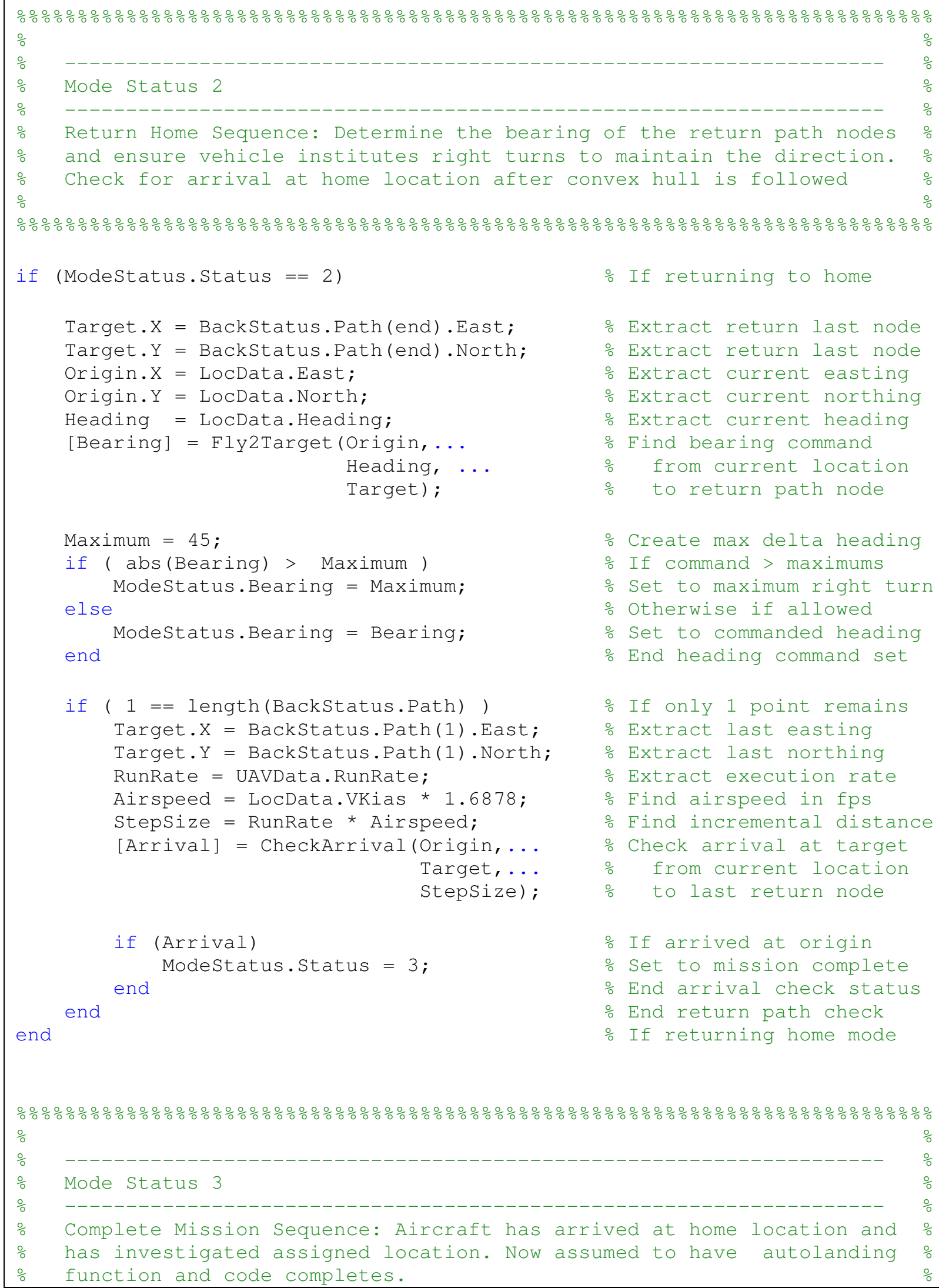




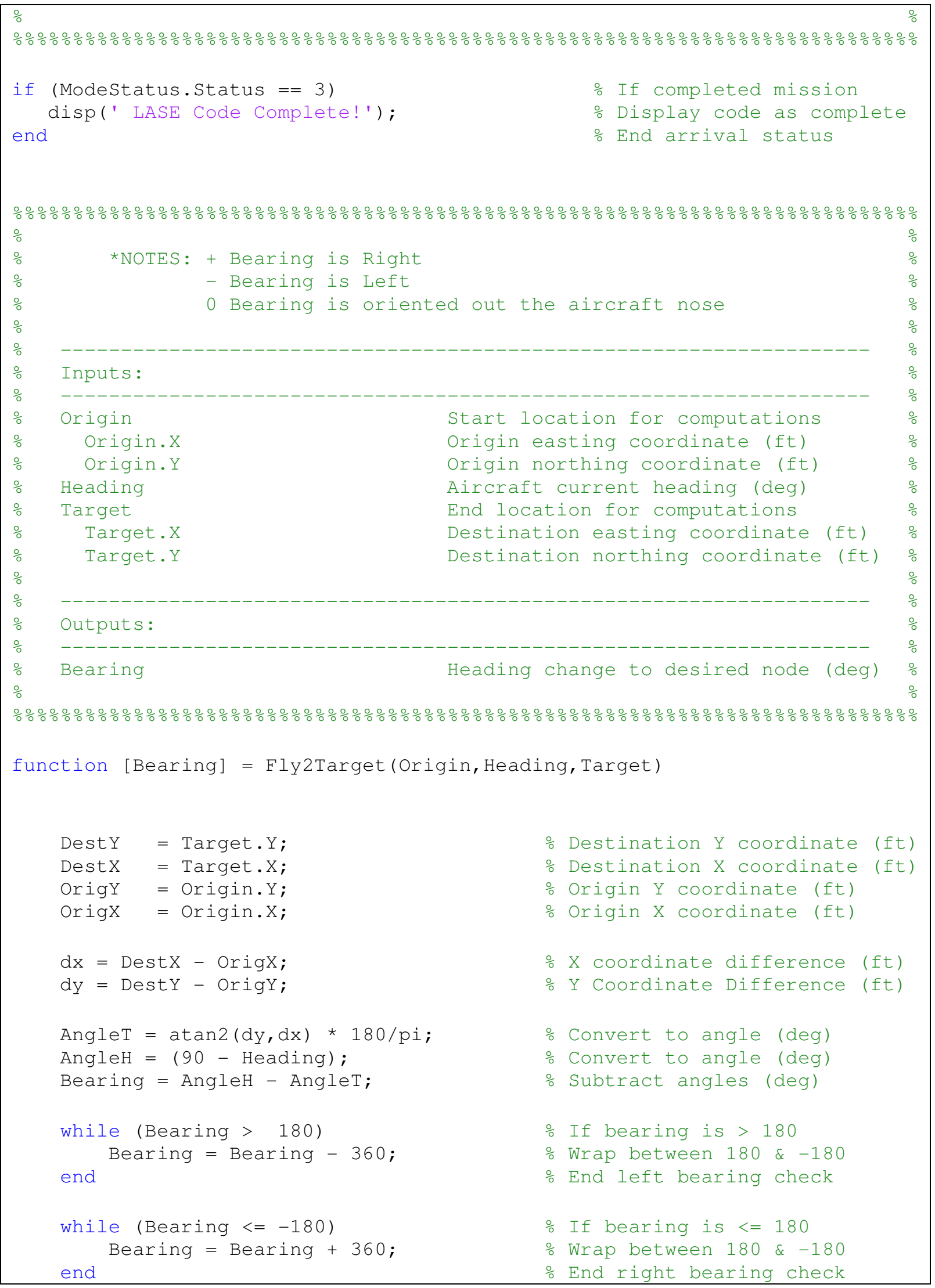




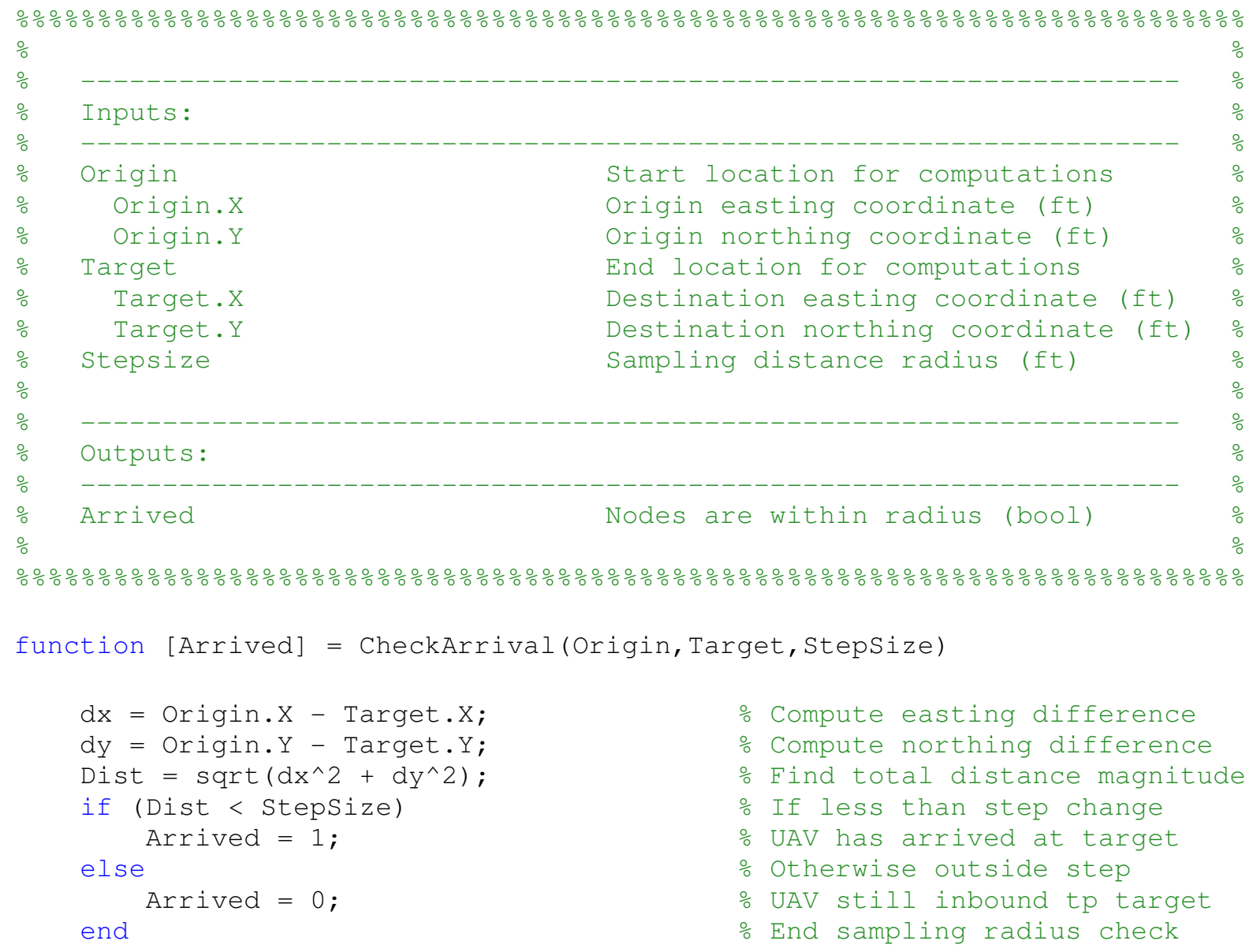

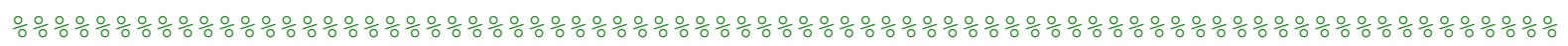

\section{LASE Code: Model_Sensor.m}

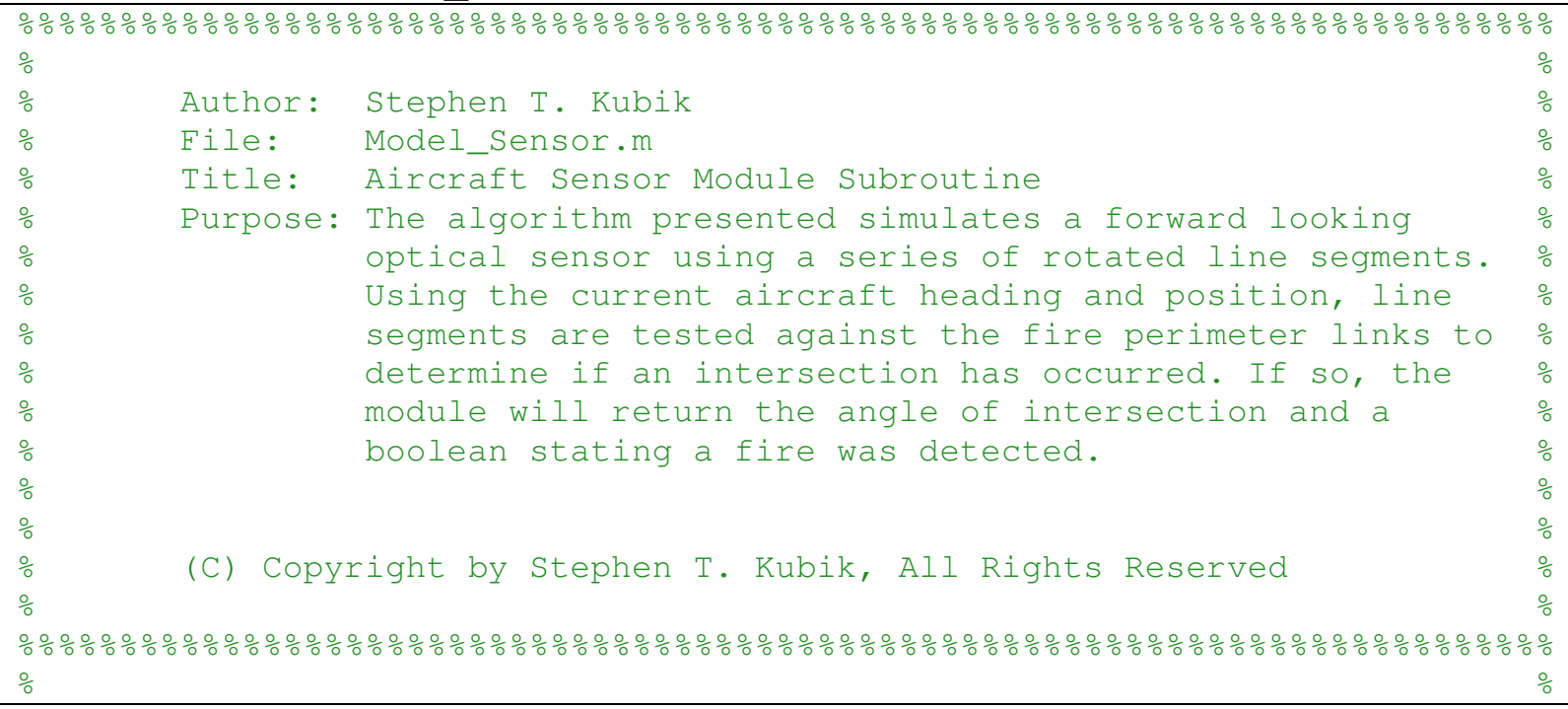




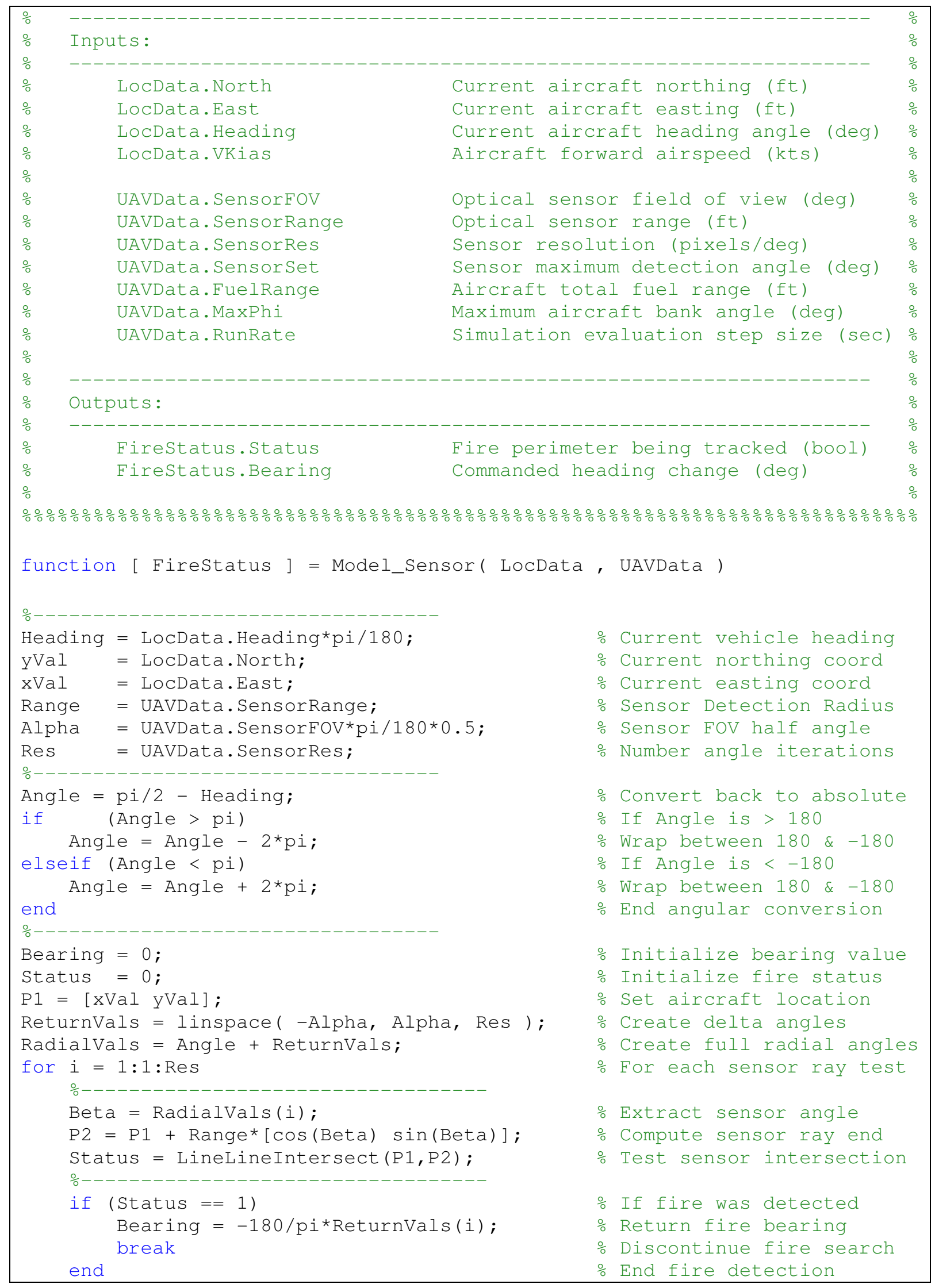




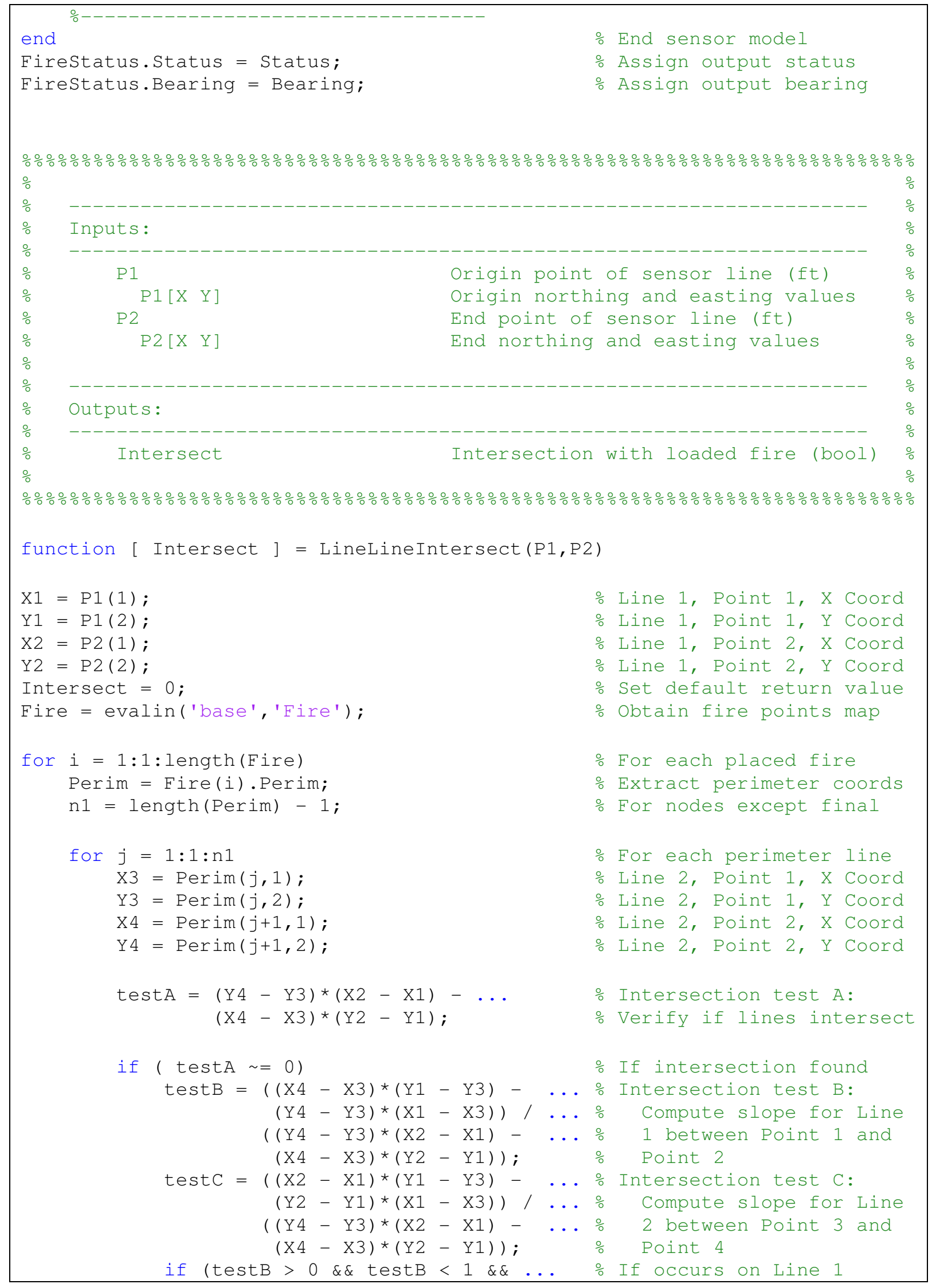




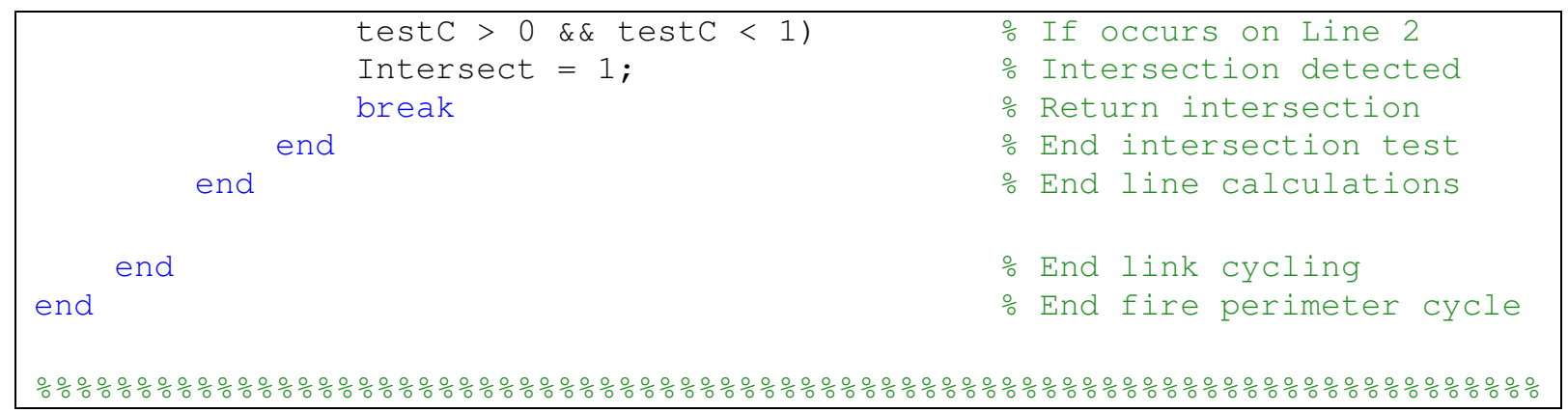

\section{LASE Code: Model_Return.m}

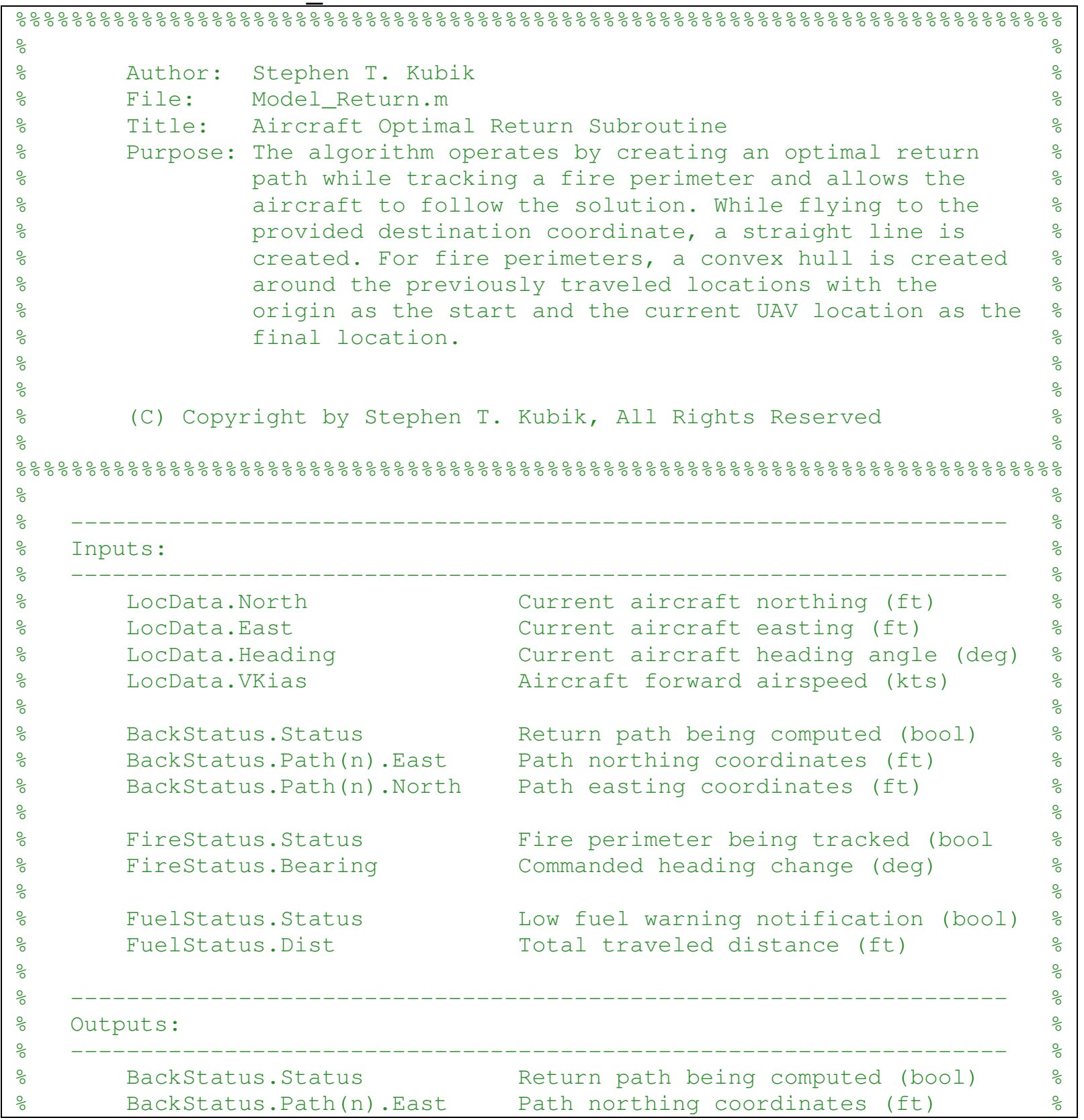




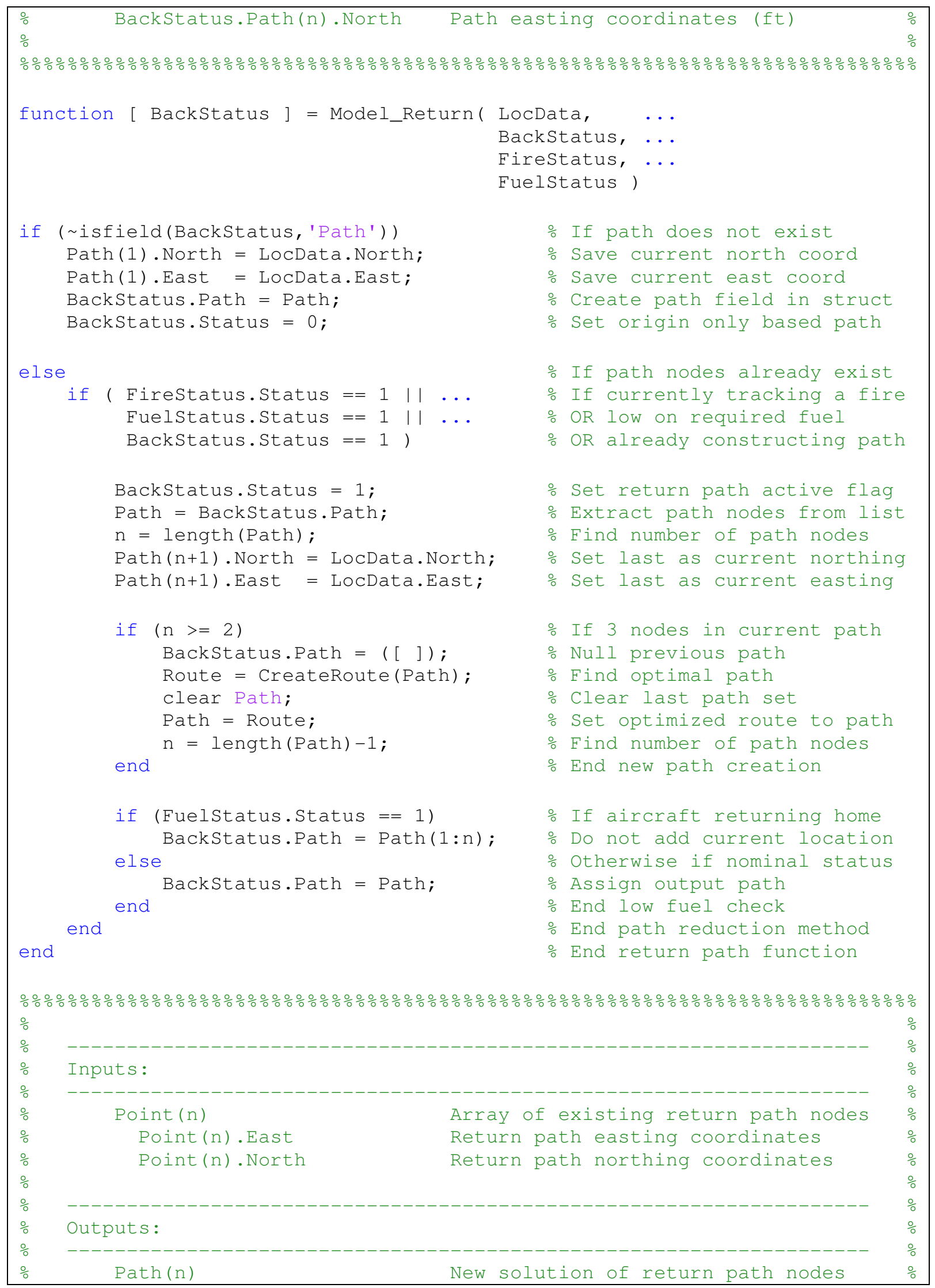




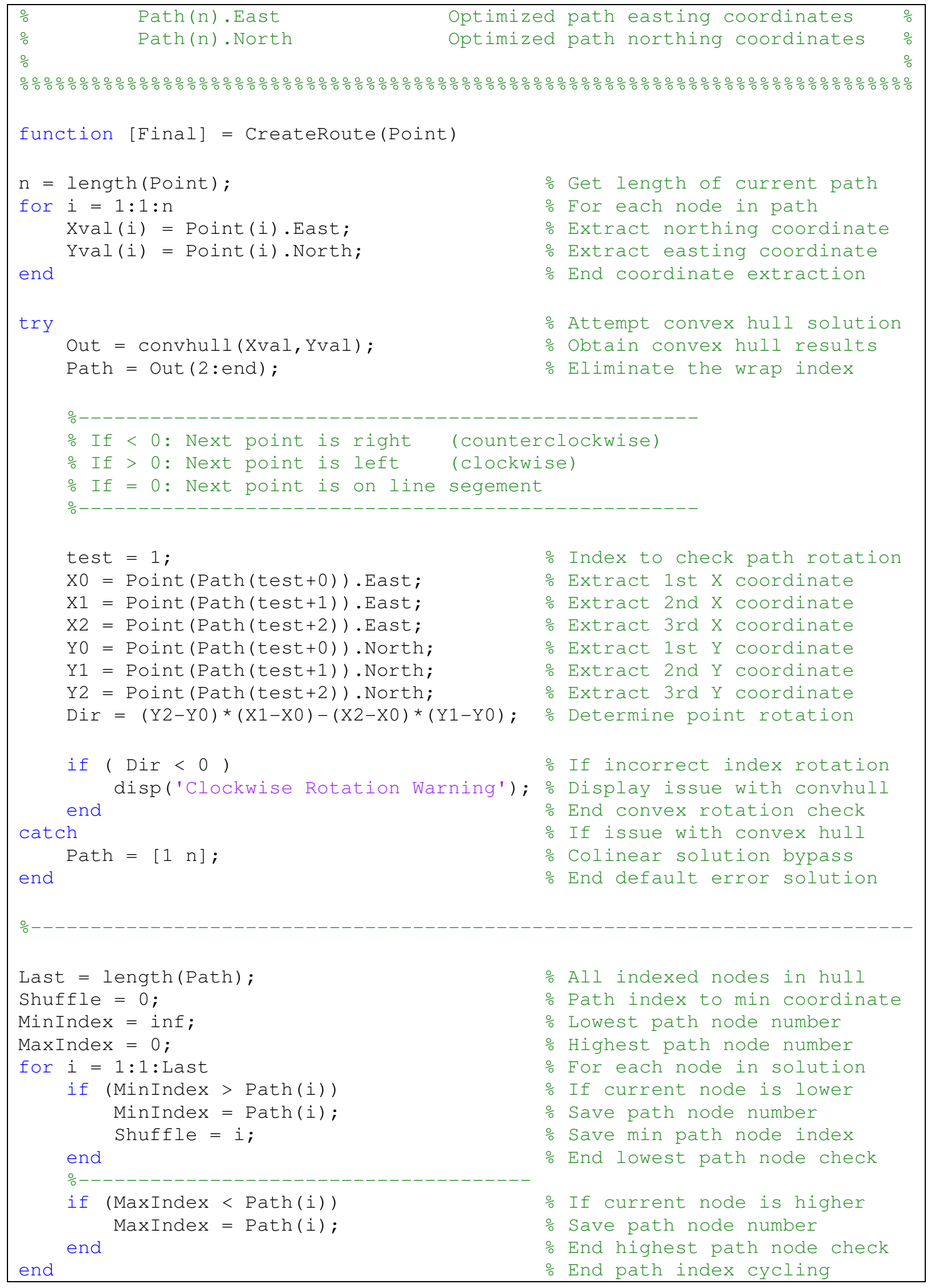




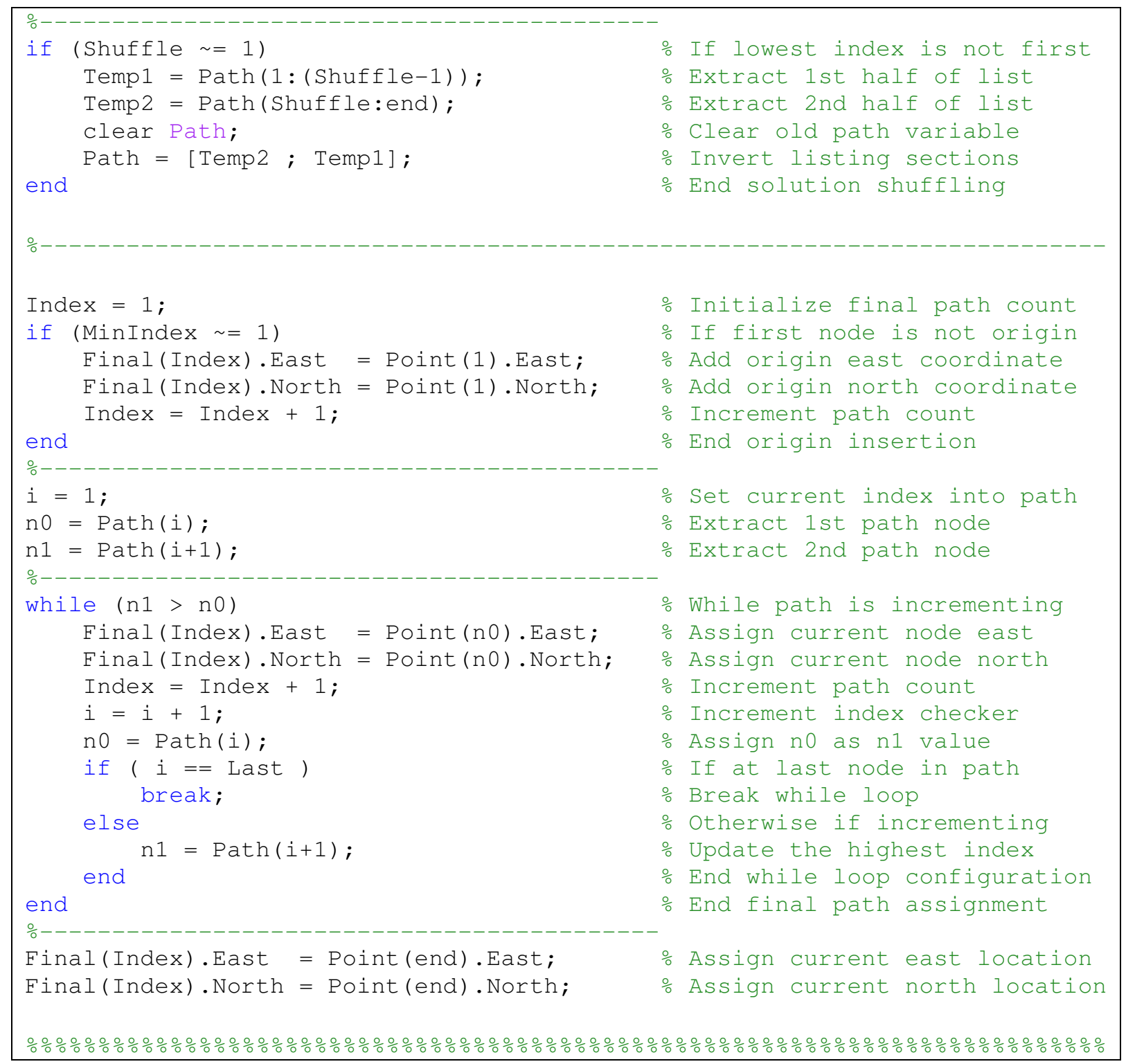

\section{LASE Code: Create Perim.m}

\begin{tabular}{|c|c|c|c|}
\hline & $\div \% \div \% \div \% \div$ & 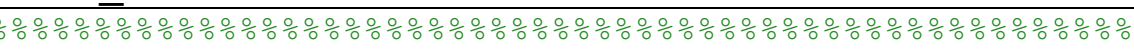 & \\
\hline$\%$ & & & $\%$ \\
\hline$\%$ & Author: & Stephen T. Kubik & $\%$ \\
\hline$\%$ & File: & Create_Perim.m & $\%$ \\
\hline$\%$ & Title: & LASE Fire Perimeter File Loader & $\%$ \\
\hline$\%$ & Purpose: & This subroutine takes user created coordinates in text & $\%$ \\
\hline$\%$ & & files and enters them into a matrix. These values & $\%$ \\
\hline$\%$ & & correspond to a single closed polygon that represents & $\%$ \\
\hline \% & & a wildfire perimeter to the code. These matrices are & 응 \\
\hline$\%$ & & then checked in the sensor model for intersection. & $\%$ \\
\hline \% & & & 응 \\
\hline 응 & & & 응 \\
\hline$\%$ & Copyr & right by Stephen T. Kubik, All Rights Reserved & 응 \\
\hline$\%$ & & & $\%$ \\
\hline
\end{tabular}




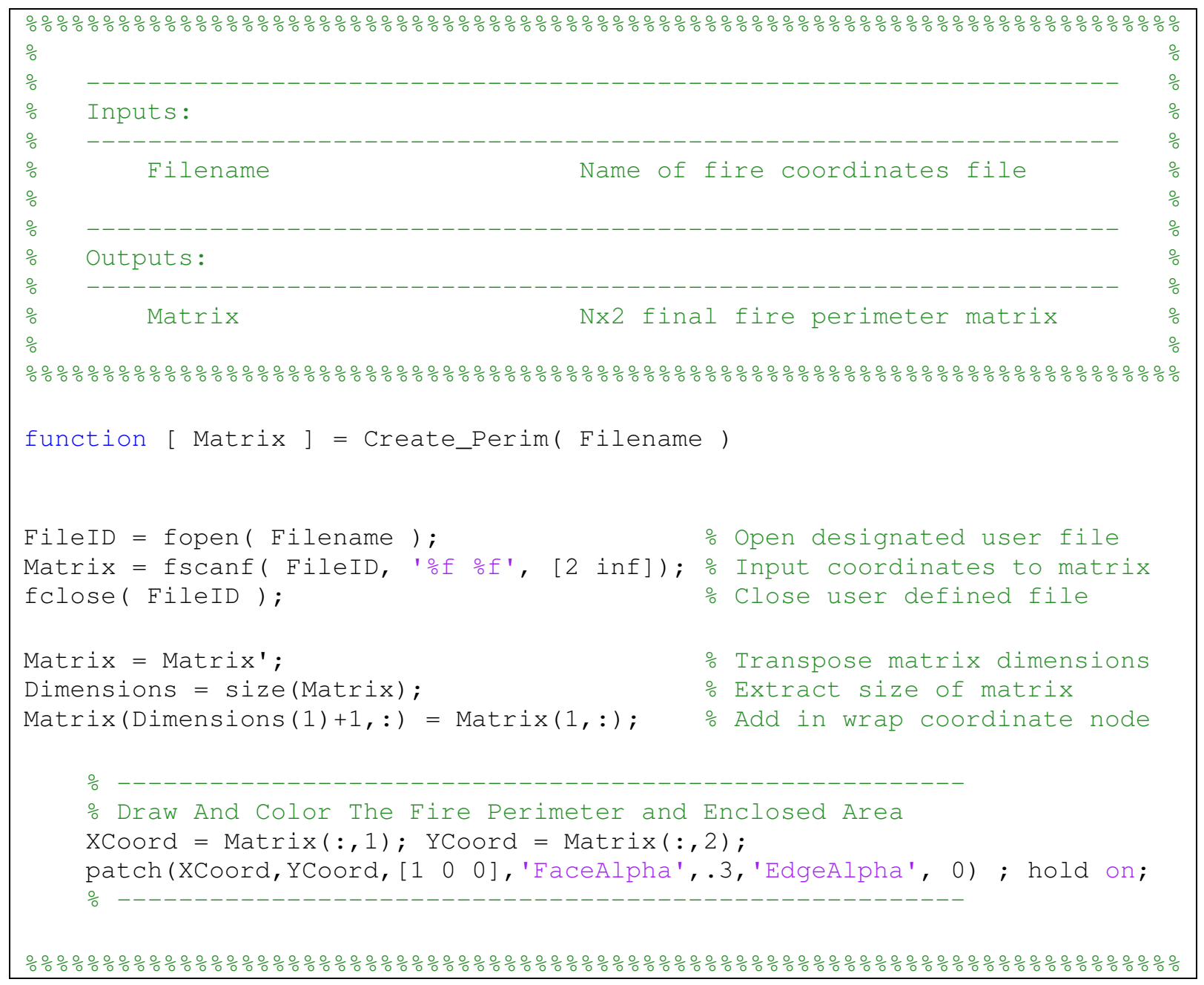




\section{APPENDIX H: HALE Matlab Simulation Code}

HALE Code: Load_HALE_Scenario.m

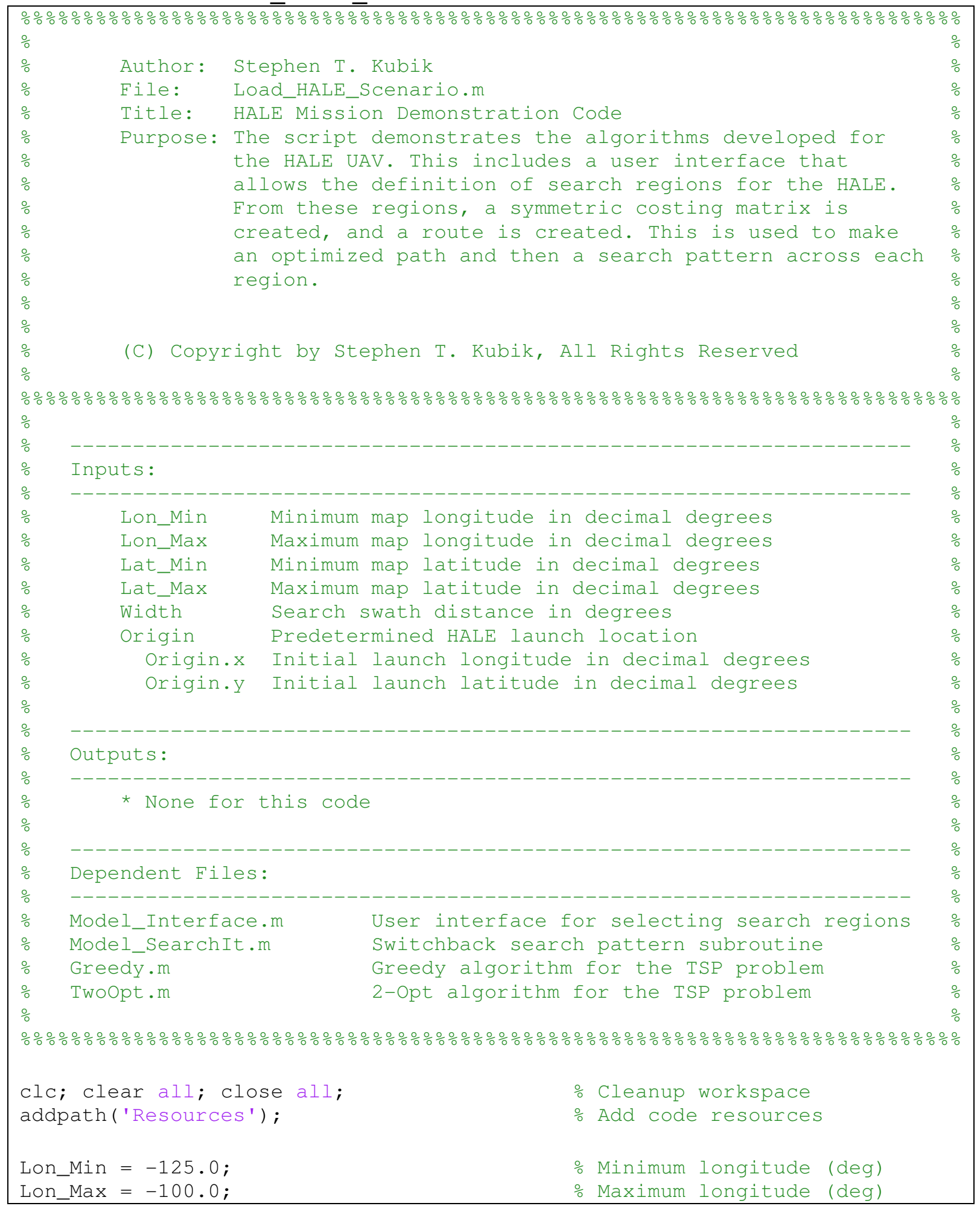




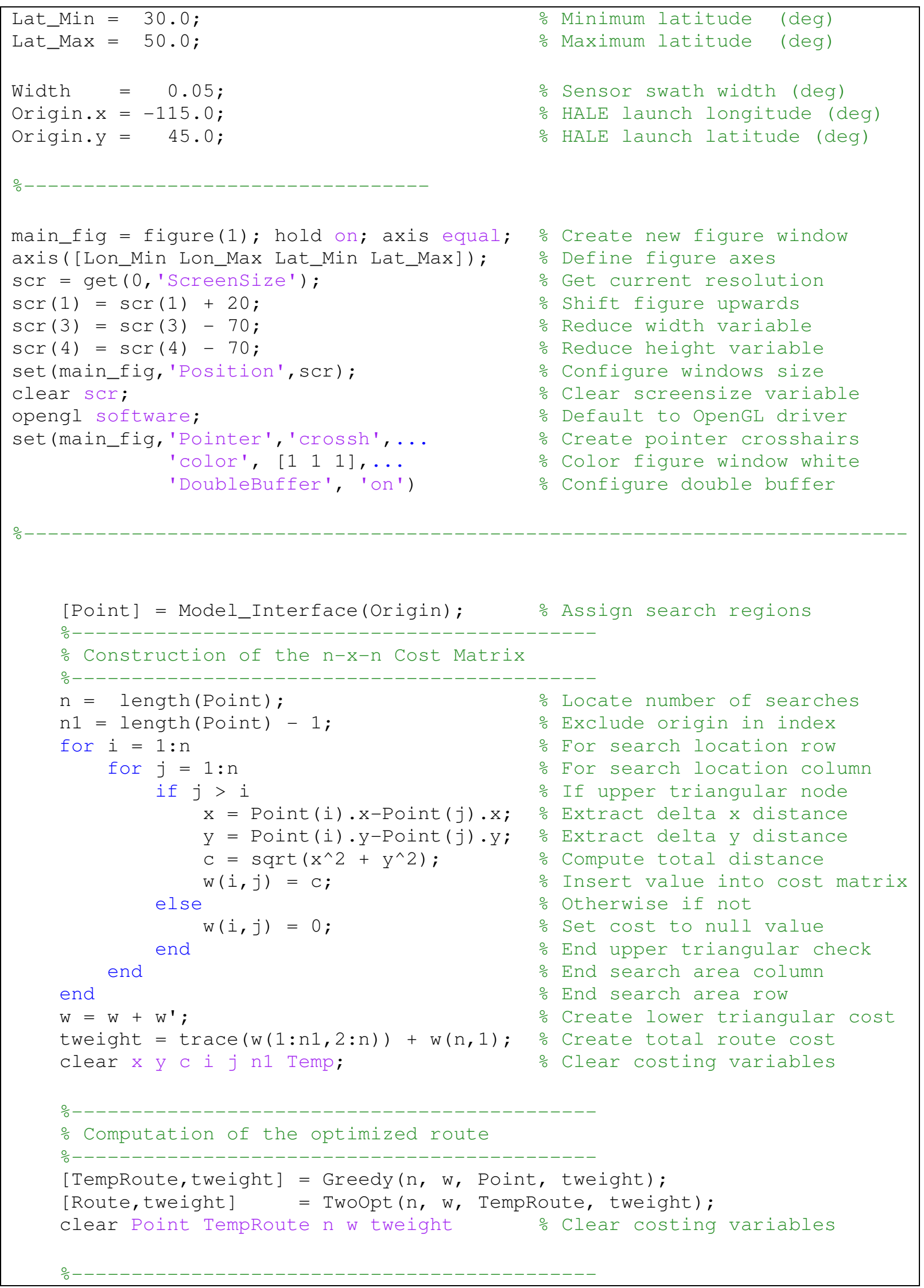




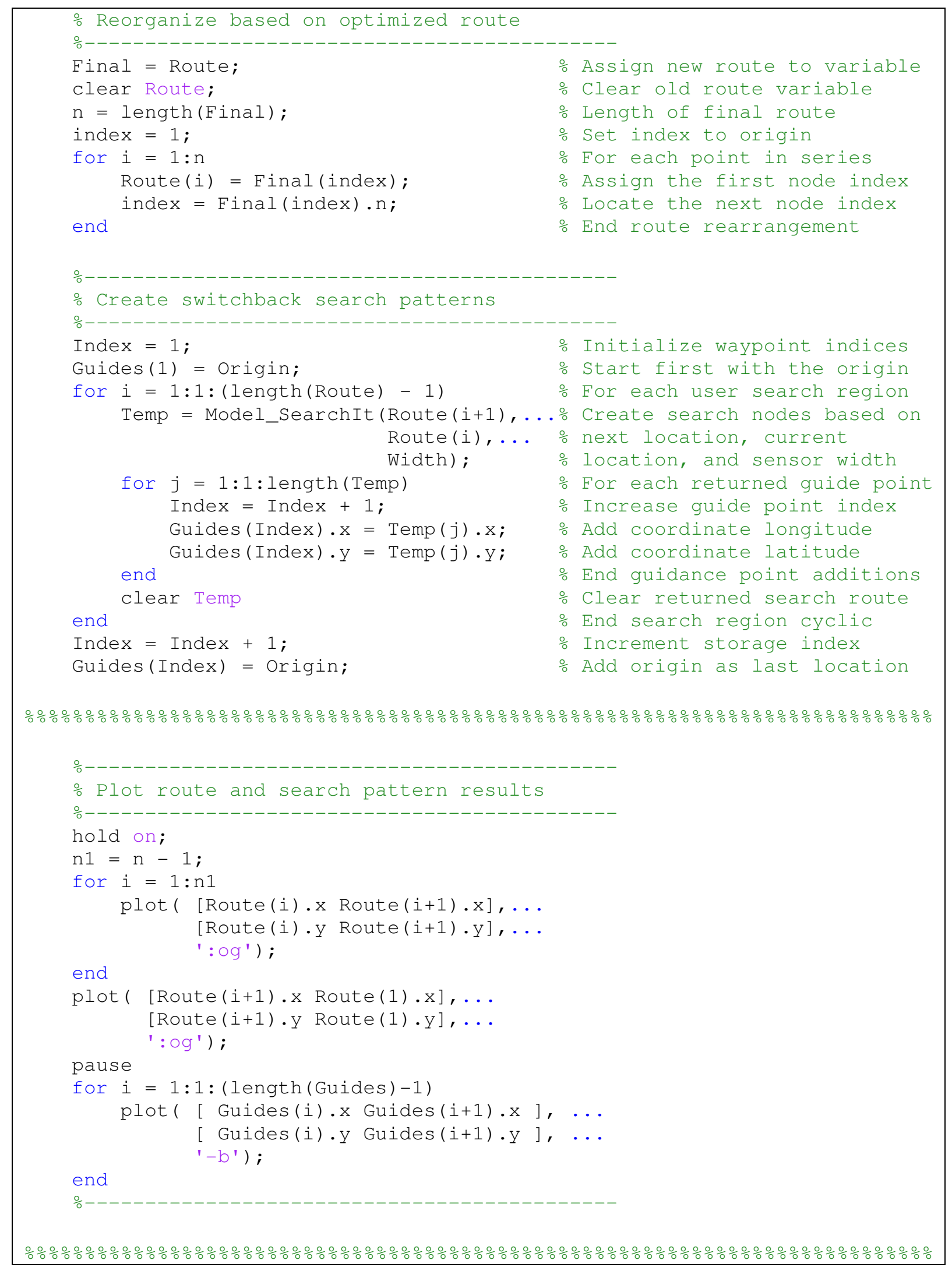




\section{HALE Code: Model Interface.m}

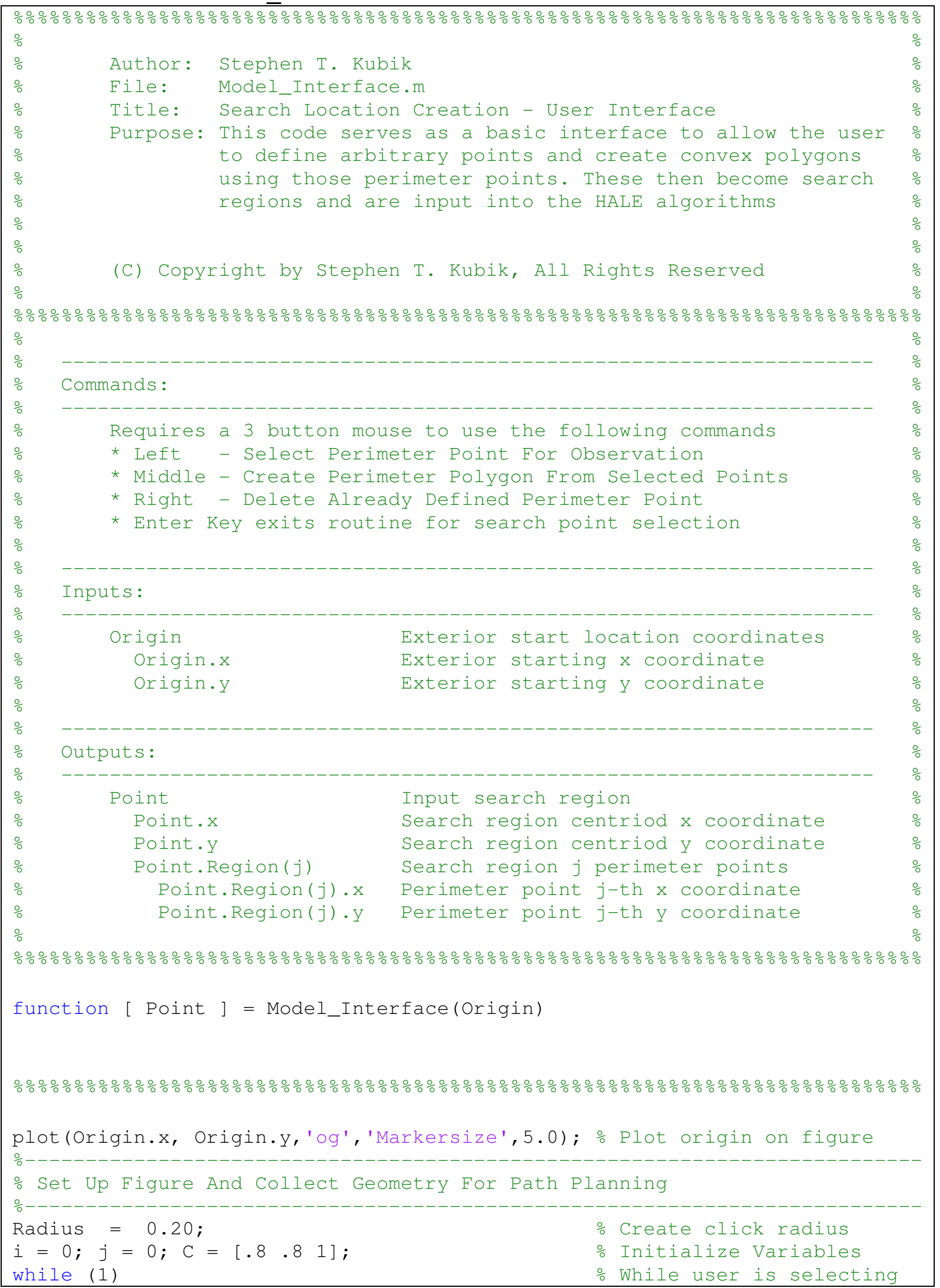




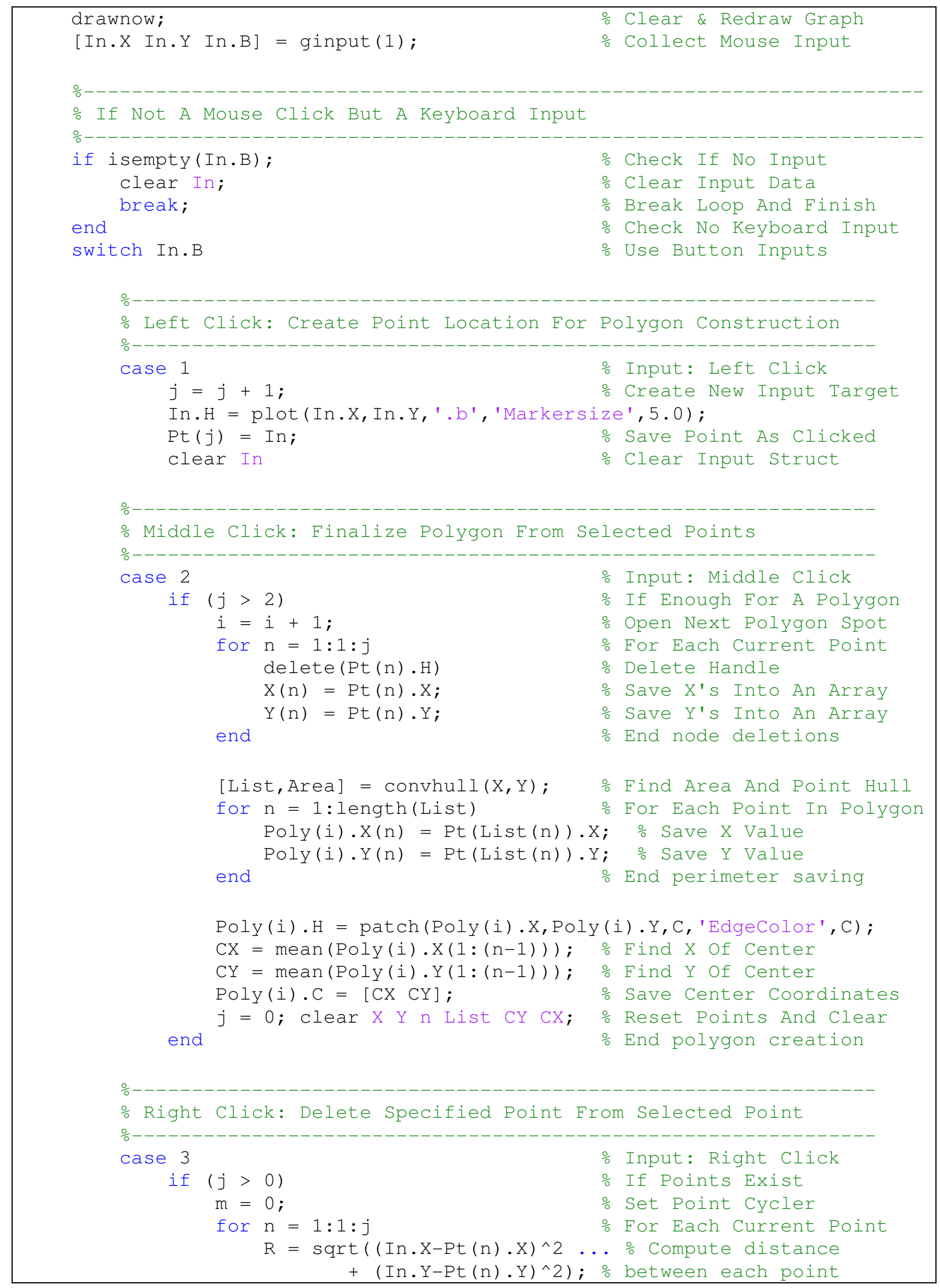






HALE Code: Model_Searchlt.m

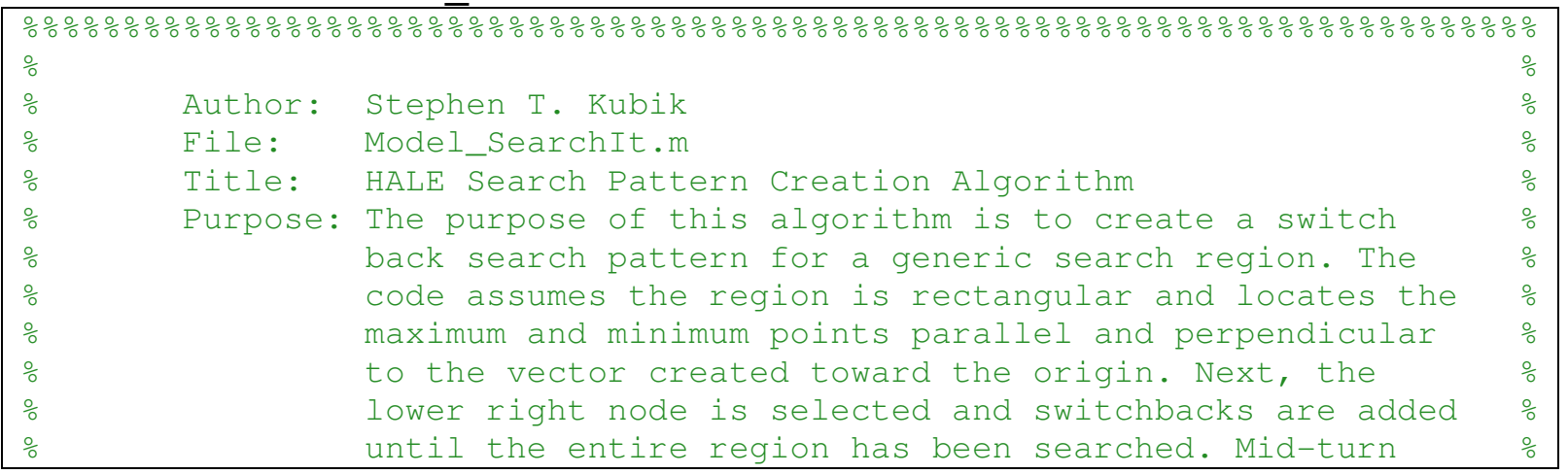




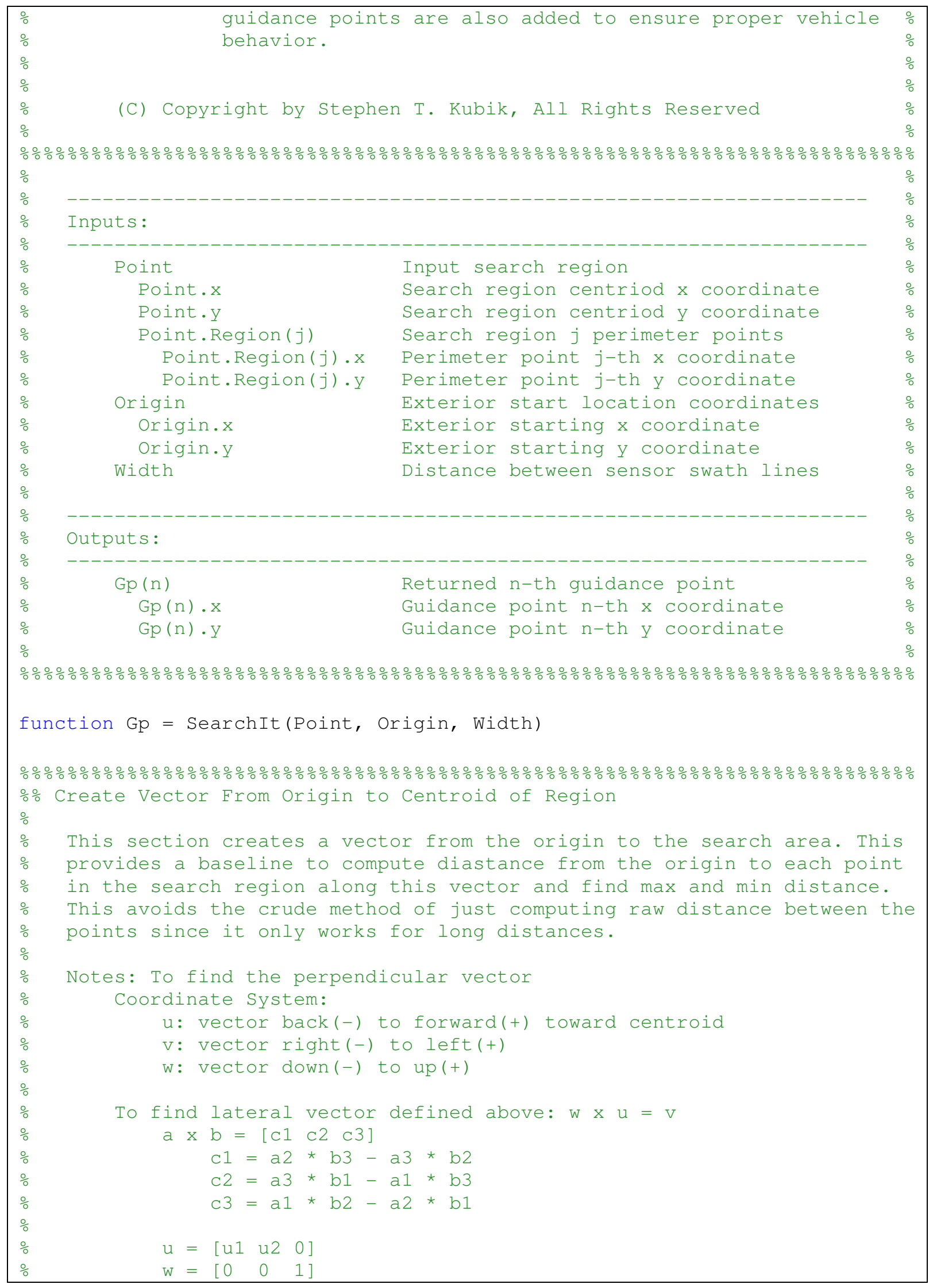




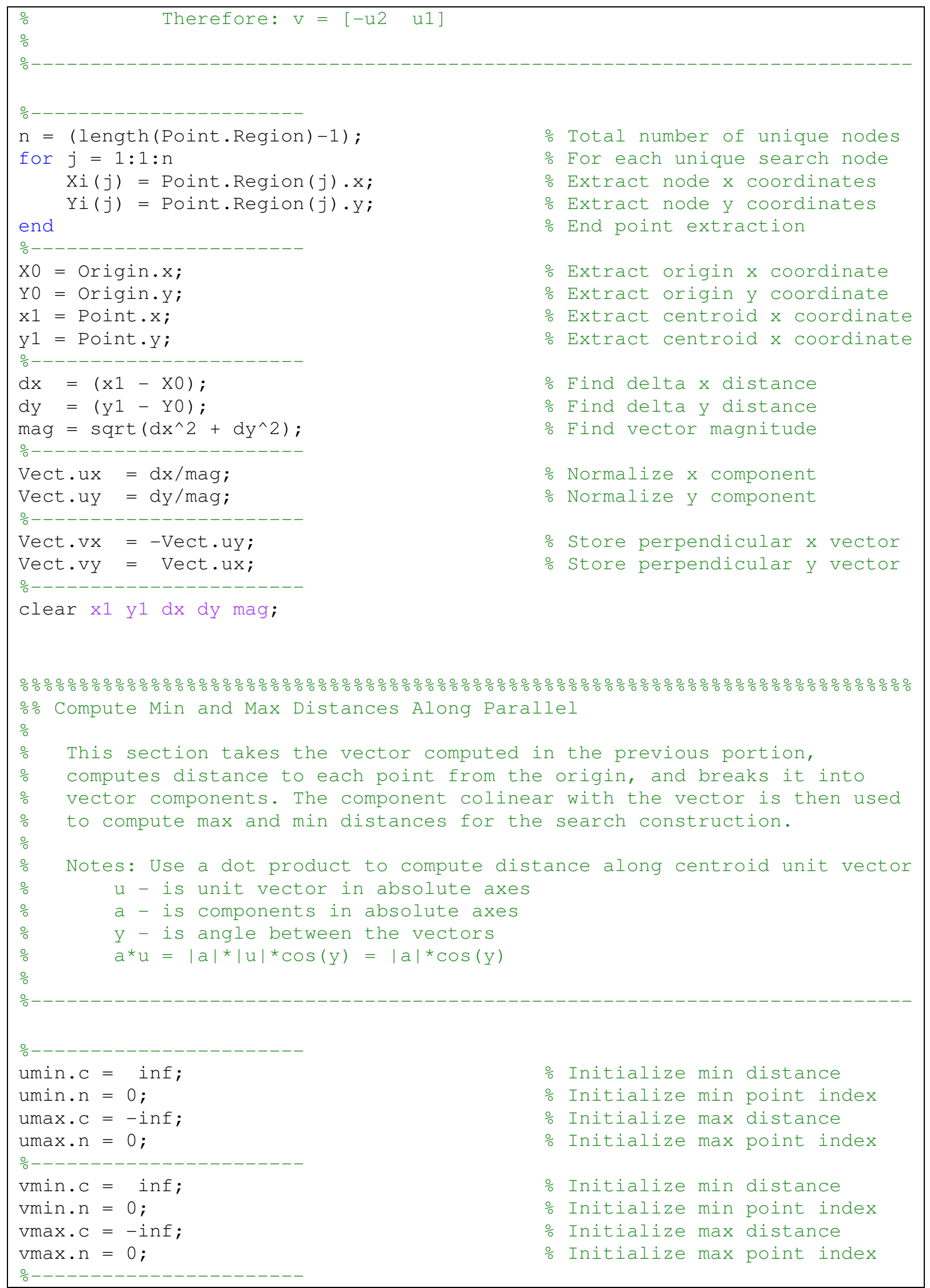




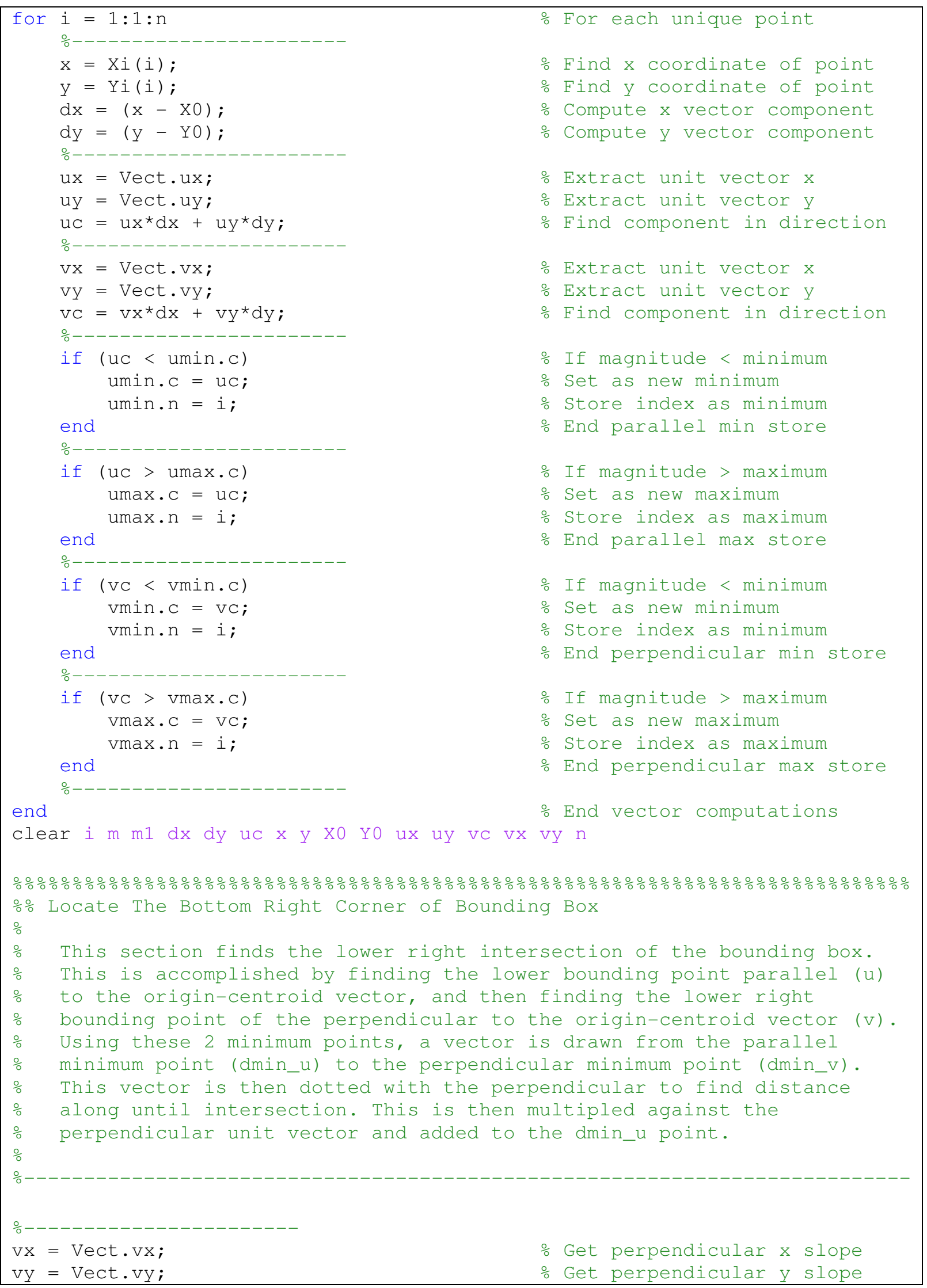




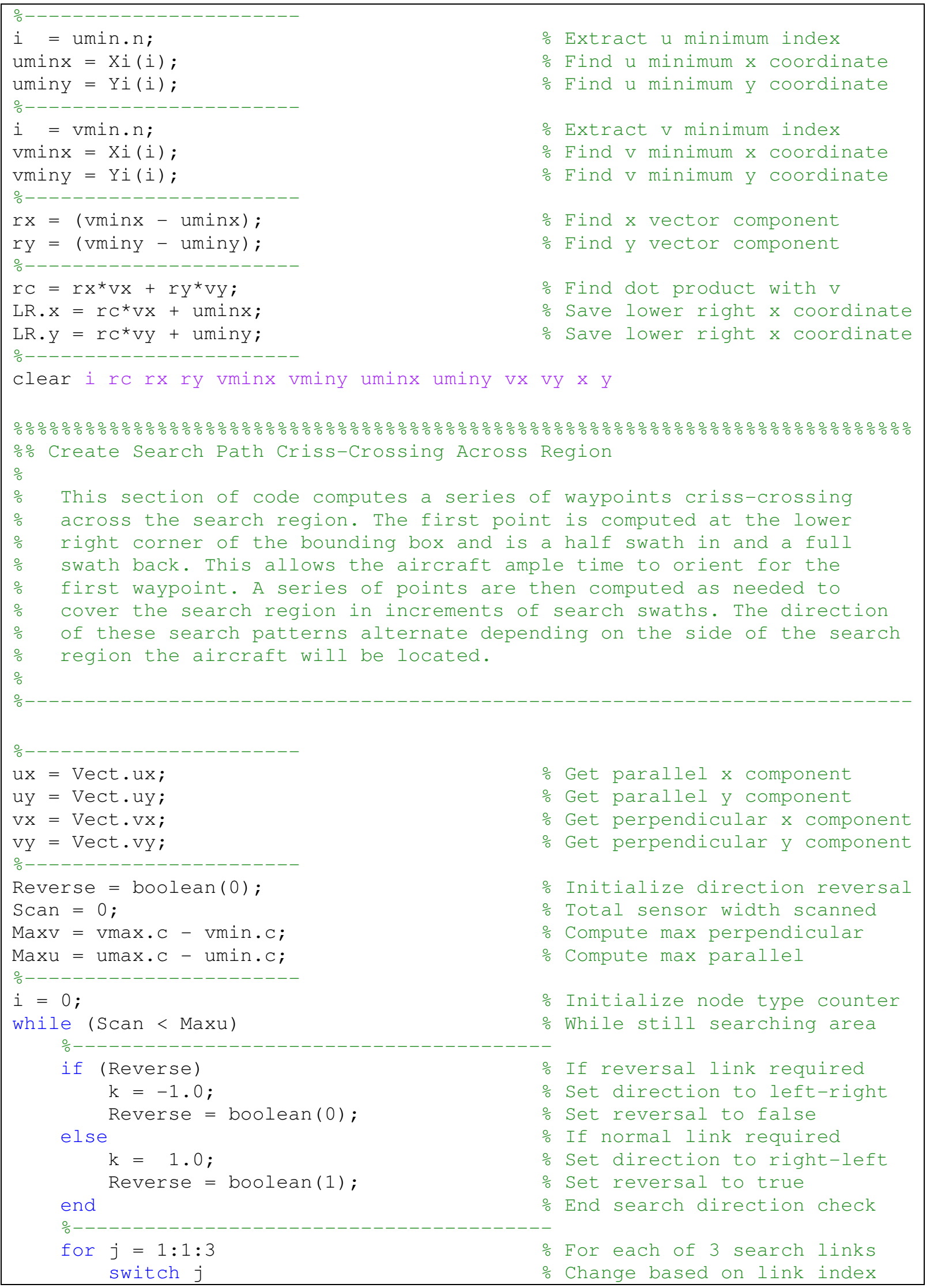




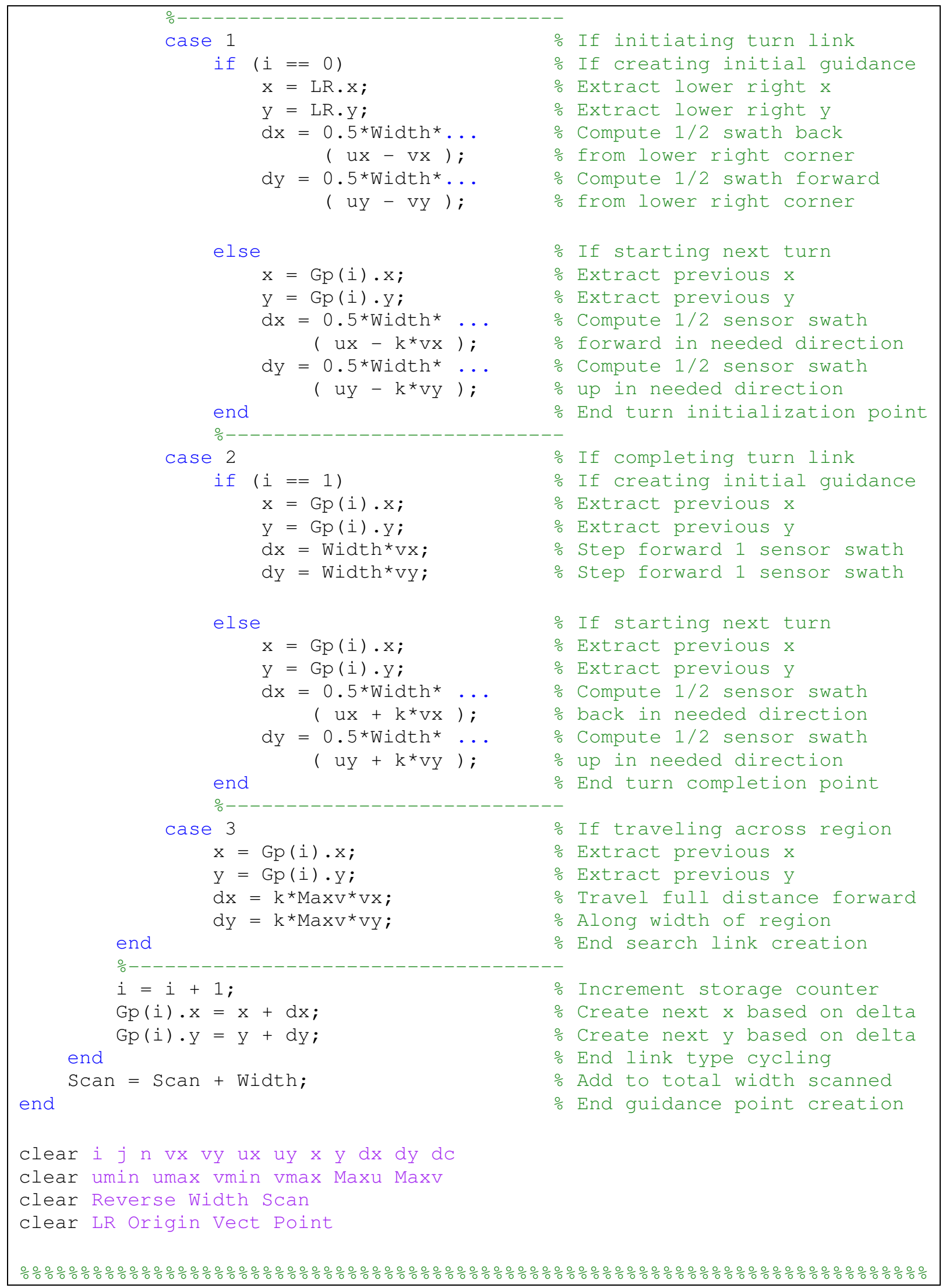

